Patrick Cadier D’Aquino e Baroni Santos

\title{
MÉTODOS PARA A ESTIMATIVA DA CAPACIDADE DE BATERIAS ESTACIONÁRIAS COM ÊNFASE EM APLICAÇÕES DE SEGURANÇA
}


Patrick Cadier D'Aquino e Baroni Santos

\title{
MÉTODOS PARA A ESTIMATIVA DA CAPACIDADE DE BATERIAS ESTACIONÁRIAS COM ÊNFASE EM APLICAÇÕES DE SEGURANÇA
}

\author{
Versão Corrigida
}

Dissertação apresentada à Escola Politécnica da Universidade de São Paulo para a obtenção do Título de Mestre em Ciências.

Área de Concentração:

Engenharia Elétrica - Sistemas Eletrônicos

Orientador: Prof. Dr. Antônio Carlos Seabra Co-Orientador: Prof. Dr. Emilio Del Moral Hernandez

São Paulo

2020 - (Edição Original)

2021 - (Versão Corrigida) 
Autorizo a reprodução e divulgação total ou parcial deste trabalho, por qualquer meio convencional ou eletrônico, para fins de estudo e pesquisa, desde que citada a fonte.

Este exemplar foi revisado e corrigido em relação à versão original, sob responsabilidade única do autor e com a anuência de seu orientador.

São Paulo, 6 de_Janeiro de 2021

Assinatura do autor:

Assinatura do orientador:

Catalogação-na-publicação

Santos, Patrick

MÉTODOS PARA A ESTIMATIVA DA CAPACIDADE DE BATERIAS

ESTACIONÁRIAS COM ÊNFASE EM APLICAÇÕES DE SEGURANÇA / P. Santos

-- versão corr. -- São Paulo, 2021.

$138 \mathrm{p}$.

Dissertação (Mestrado) - Escola Politécnica da Universidade de São Paulo. Departamento de Engenharia de Sistemas Eletrônicos.

1.BATERIAS ELÉTRICAS 2.REDES NEURAIS 3.FUZZY (INTELIGÊNCIA ARTIFICIAL) I.Universidade de São Paulo. Escola Politécnica. Departamento de Engenharia de Sistemas Eletrônicos II.t. 


\section{AGRADECIMENTOS}

Ao professor Antônio Carlos Seabra, meu orientador, pela ajuda e paciência durante o mestrado.

Ao professor Emilio Del Moral Hernandez, meu co-orientador, pelas ideias e propostas que ajudaram esse trabalho.

Aos participantes do grupo ICONE pelas dicas.

À Cássia, minha esposa que me ajudou a persistir estudando.

Aos meus pais, Irene e Cassio, que me incentivaram sempre a estudar.

À Laura, minha filha que mesmo tão nova já me dá forças para continuar. 


\section{RESUMO}

Neste projeto, é proposto métodos diferentes para medir a capacidade e o envelhecimento de baterias estacionárias com ênfase em aplicações de segurança. Para tanto, é primeiro descrito a importância de baterias em diversas aplicações, principalmente as baterias de íons de lítio. Um estudo das características elétricas e químicas de baterias de íons de lítio é apresentado, mostrando entre outras coisas as causas eletroquímicas do envelhecimento.

Além disso, as diferentes técnicas para o monitoramento de sistemas são mostradas. Neste projeto pretende-se usar redes neurais, lógica fuzzy e adaptatividade para estimar a capacidade de uma bateria usando os valores da resistência e capacitância interna. Os dados recolhidos são apresentados e analisados. Os diferentes métodos são comparados e as vantagens e desvantagens de cada método são discutidas.

Palavras-chave: Baterias elétricas. Redes Neurais. Lógica Fuzzy. 


\begin{abstract}
This project proposes different methods for measuring the capacity and aging of stationary batteries on security sistems. For this, it is first described batteries' important role on many applications, especially concerning lithium-ion batteries. A study of electrical and chemical characteristics of lithium-ion batteries is presented, showing among other things the electrochemical causes for aging.

Furthermore, different techniques for Battery Management System (BMS) are shown. In this project we intend to use a neural network, fuzzy logic and adaptability to estimate the battery's capacity using the internal resistance and capacitance values. The data collected is presented and analyzed. In the end, each method is compared with one another.
\end{abstract}

Keywords: Electrical batteries. Neural networks. Fuzzy logic. 


\section{LISTA DE FIGURAS}

Figura 1 - Crescimento do mercado de baterias de íons de lítio. .............................17

Figura 2 - Crescimento do mercado de baterias de lítio........................................18

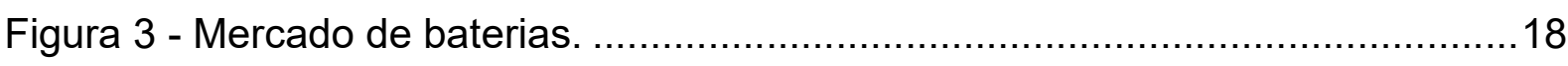

Figura 4 - Comparação da densidade energética entre tecnologias. .......................20

Figura 5 - Comparação da densidade energética entre tecnologias. .......................20

Figura 6 - Comparação do número de ciclos entre tecnologias. ..............................21

Figura 7 - Evolução do preço em $U \$ \$ / W h$ de diferentes formatos...........................21

Figura 8 - Número de artigos sobre baterias de íons de lítio por país.....................22

Figura 9 - Formatos de bateria: a - cilíndrico. b - prismático. c - laminado ou pouch.25

Figura 10 - Estrutura do catodo de Lítio-Cobalto...............................................26

Figura 11 - Estrutura do catodo de Lítio-Manganês. ...........................................26

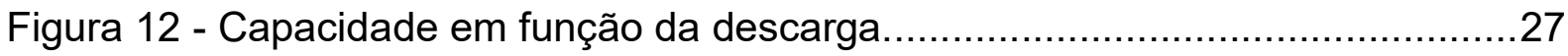

Figura 13 - Capacidade em função do número de ciclos. .........................................29

Figura 14 - Evolução do SOH em função da carga e da temperatura........................30

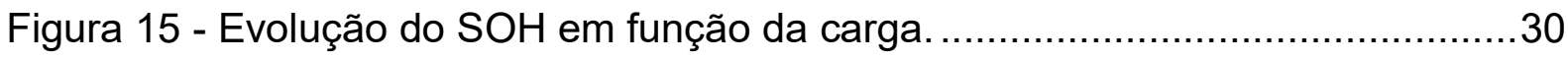

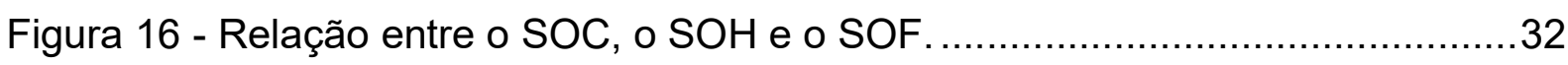

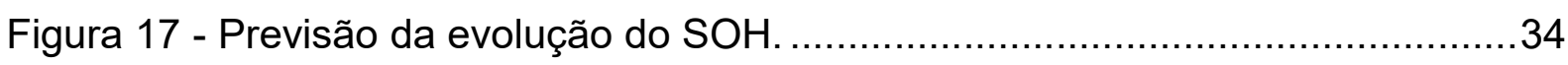

Figura 18 - Evolução do SOH em função da tensão máxima de carga......................35

Figura 19 - Evolução da Capacidade em função da temperatura e SOC....................36

Figura 20 - Evolução da Capacidade em função da corrente de descarga.................38

Figura 21 - Modelo de Randle de uma bateria..................................................38

Figura 22 - Circuito de proteção em bateria de íons de lítio.......................................39

Figura 23 - Circuito de proteção da bateria de íons de lítio......................................40

Figura 24 - Circuito modelo do teste para estimar os parâmetros da bateria............42

Figura 25 - Tensão na saída da bateria durante o teste........................................42

Figura 26 - Circuito equivalente para o cálculo da resistência de Thévenin..............43

Figura 27 - Circuito equivalente de Thévenin......................................................44

Figura 28 - Comparação entre a simulação e os valores medidos............................46

Figura 29 - Circuito de carga das baterias. .......................................................

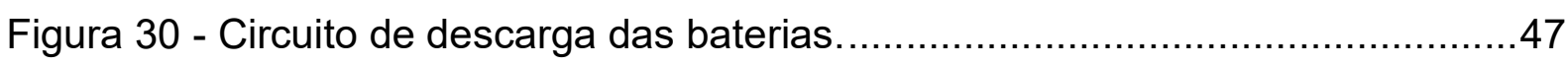

Figura 31 - Foto dos 3 tipos de baterias testadas. ............................................48

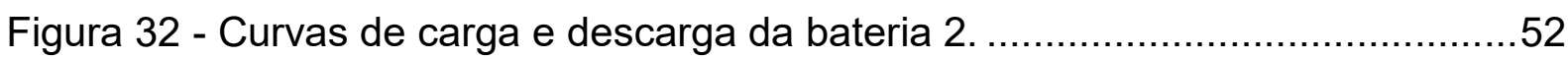

Figura 33 - Curvas do início da descarga da bateria 2......................................53 
Figura 34 - Relação entre a capacitância e o SOH.

Figura 35 - Relação entre a resistência Rx e o SOH.

Figura 36 - Relação entre a resistência Rx e o SOH dividido por modelo de bateria.

Figura 37 - Relação entre a capacitância e o SOH dividido por modelo de bateria. . 55 Figura 38 - Evolução do SOH, da resistência Rx e da capacitância ciclo a ciclo da bateria 1 .

Figura 39 - Evolução do SOH, da resistência Rx e da capacitância ciclo a ciclo da bateria 3 .

Figura 40 - Modelo de um perceptron de uma rede neural. 61

Figura 41 - llustração do método de gradiente decrescente. 62

Figura 42 - Rede neural com uma camada interna. .63

Figura 43 - formas de funções de pertencimento: a) trapezoidal, b) triangular, c) formato de sino e d) guassiana,

Figura 44 - Componentes de um sistema de lógica fuzzy, .65

Figura 45 - Funções de pertencimento triangulares para a análise da resistência....66 Figura 46 - Funções de pertencimento triangulares para a análise da capacitância. 66 Figura 47 - Funções de pertencimento gaussianas para a análise da resistência. ...67 Figura 48 - Funções de pertencimento gaussianas para a análise da capacitância. 67 Figura 49 - Topologia da rede neural. .75

Figura 50 - Gráfico do erro médio da rede neural por faixa de valor de $\mathrm{SOH}$. .76

Figura 51 - Gráfico do erro médio no modelo de Sugeno de ordem 0 por faixa de valor de $\mathrm{SOH}$.

Figura 52 - Gráfico do erro médio no modelo de Sugeno de ordem 1 por faixa de valor de $\mathrm{SOH}$. .80

Figura 53 - Diminuição do erro médio da bateria 3 para cada dado novo. .83

Figura 54 - Diminuição do erro médio da bateria 5 para cada dado novo. 83

Figura 55 - Diminuição do erro médio da bateria 11 para cada dado novo. .84

Figura 56 - Diminuição do erro médio da bateria 17 para cada dado novo.

Figura 57 - Diminuição do erro médio da bateria 3 na segunda versão do algoritmo.

Figura 58 - Diminuição do erro médio da bateria 5 na primeira versão do algoritmo. 
Figura 59 - Diminuição do erro médio da bateria 5 na segunda versão do algoritmo.

Figura 60 - Comparação da diminuição do erro médio para o $2^{\circ}$ e $3^{\circ}$ algoritmo com a bateria 10 . 89

Figura 61 - Topologia da segunda rede neural para previsão do EOL....................90

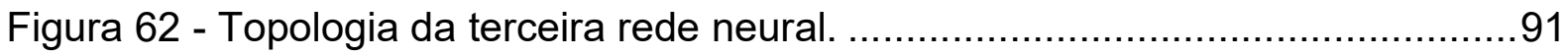

Figura 63 - Gráfico do erro médio no $1^{\circ}$ modelo linear por faixa de valor. ................93

Figura 64 - Gráfico do erro médio no $2^{\circ}$ modelo linear por faixa de valor. ................95

Figura 65 - Gráfico do erro médio no $3^{\circ}$ modelo linear por faixa de valor. ................96 


\section{LISTA DE TABELAS}

Tabela 1 - Registro das tensões da bateria nos primeiros $400 \mathrm{~ms}$ de teste exemplo 45

Tabela 2 - Valores estimados do modelo de Randle de um teste exemplo .45

Tabela 3 - Definição do fator de ciclo da descarga. .............................................50

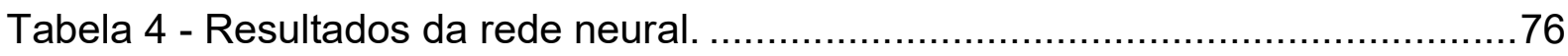

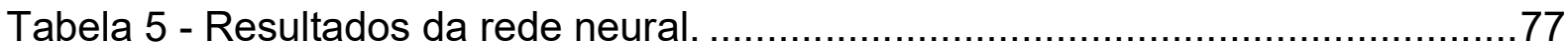

Tabela 6 - Resultados do modelo de Sugeno de ordem 0 ……..........................78

Tabela 7 - Resultados do modelo de Sugeno de ordem 0 . ..................................79

Tabela 8 - Resultados do modelo de Sugeno de ordem 1 . ....................................... 80

Tabela 9 - Resultados do modelo de Sugeno de ordem 0 ................................... 81

Tabela 10 - Erros médios da rede inicial e da rede adaptativa...............................82

Tabela 11 - Erros médios da rede inicial e da segunda versão da rede adaptativa. . 86

Tabela 12 - Erros médios da rede inicial e da rede adaptativa final. ........................88

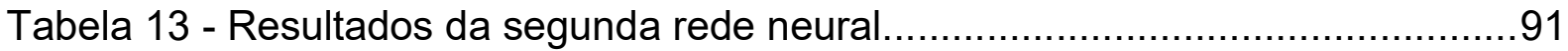

Tabela 14 - Comparando os resultados da primeira e terceira redes neurais...........92

Tabela 15 - Resultados do $1^{\circ}$ modelo linear.........................................................93

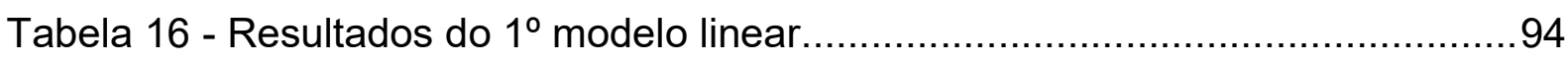

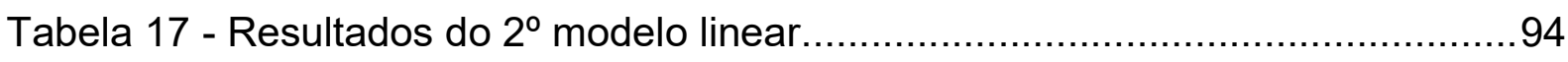

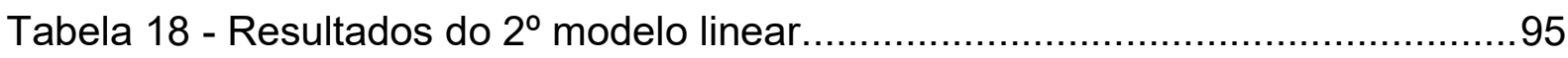

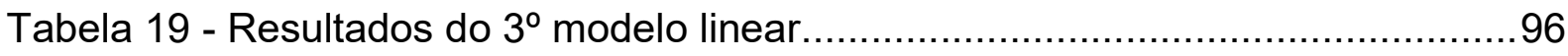

Tabela 20 - Resultados do $3^{\circ}$ modelo linear.......................................................

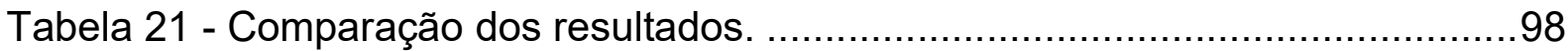

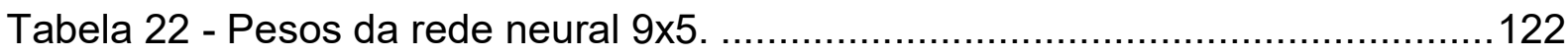

Tabela 23 - Regras do modelo de Sugeno de ordem 0 ....................................124

Tabela 24 - Regras do modelo de Sugeno de ordem 1, regras para $R_{\mathrm{i}}$................. 125

Tabela 25 - Regras do modelo de Sugeno de ordem 1, regras para $\mathrm{C}_{\mathrm{i}}$................126

Tabela 26 - Regras do modelo de Sugeno de ordem 1, regras para bi.................127

Tabela 27 - Pesos da segunda rede neural....................................................128

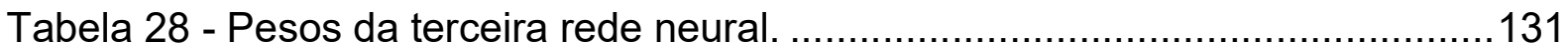

Tabela 29 - Relação dos modelos de baterias utilizados. ...................................133

Tabela 30 - Dados obtidos.........................................................................134 
LISTA DE SIGLAS E ABREVIATURAS

$A C / D C$

Alternating Current / Direct Current

ADC

Analog to Digital Converter

AHC

Capacidade nominal em Ah

ARM

Advanced RISC Machine

BMS

Battery Management System

CID

Circuit Interrupt Device

CDL

Capacitância de Dupla Camada

EEPROM

Electrically Erasable Programmable Read-Only Memory

EIS

Electrochemical Impedance Spectroscopy

EOL

End Of Life

EV

Electric Vehicle

FW

Firmware

HEV

Hybrid Electric Vehicle

MBP

Multiple Back-Propagation

MOSFET

Metal-Oxide-Semiconductor Field-Effect Transistor

PC

Personal Computer

PDF

Probability Density Function

PF

Particle Filtering

PHM

Prognostics and Health Management

PN

Part Number

PoF

Physics Of Failure

PTC

Positive Thermal Coefficient 


$\begin{array}{ll}\text { PWM } & \text { Pulse Width Modulation } \\ \text { RCT } & \text { Resistência de Transferência de Carga } \\ \text { RE } & \text { Resistência do Eletrólito. } \\ \text { RUL } & \text { Remaining Useful Life } \\ \text { RVM } & \text { Relevance Vector Machine } \\ \text { RW } & \text { Resistência de Warburg } \\ \text { SCA } & \text { Self-Clustering Algorithm } \\ \text { SOC } & \text { State Of Charge ou estado da carga } \\ \text { SOF } & \text { State of Function ou estado de funcionalidade } \\ \text { SOH } & \text { State Of Health ou estado de saúde } \\ \text { SOL } & \text { State of Life } \\ \text { SW } & \text { Software } \\ \text { USB } & \text { Universal Serial Bus }\end{array}$




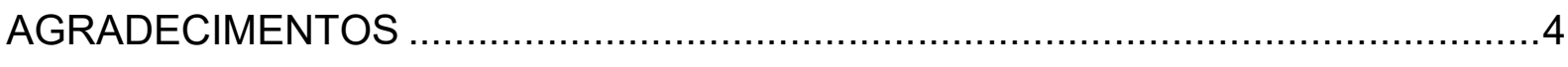

RESUMO

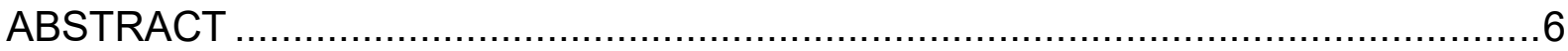

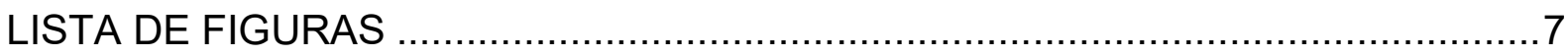

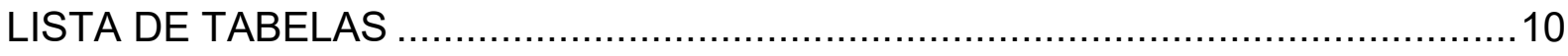

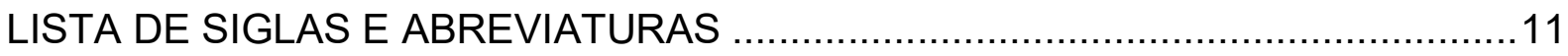

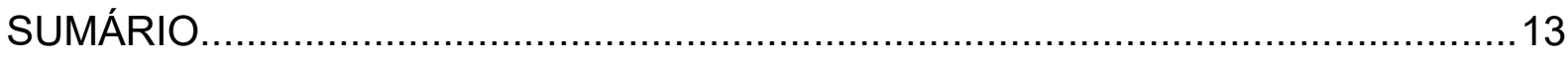

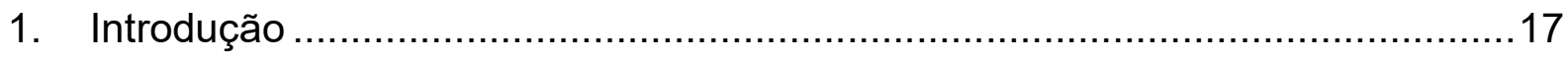

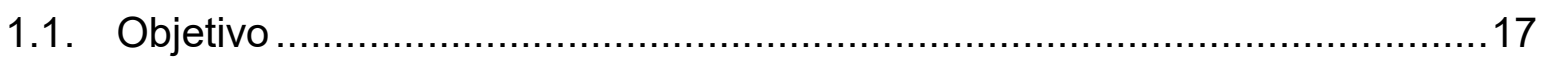

1.2. Relevância das baterias estacionárias ..................................................17

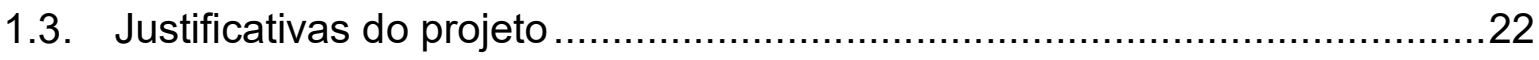

2. Modelos e parâmetros das baterias de íons de lítio ........................................25

2.1. Descrição de uma bateria de lítio ("rocking chair") ....................................25

2.2. Descrição dos parâmetros de medição da bateria .....................................27

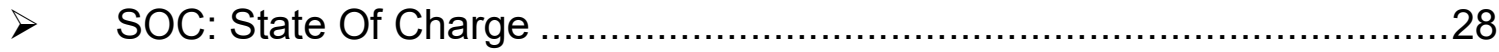

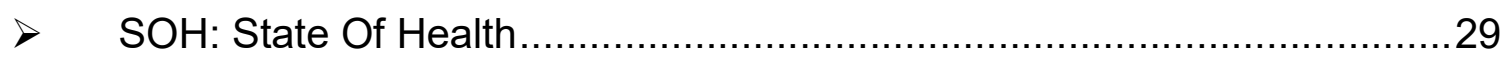

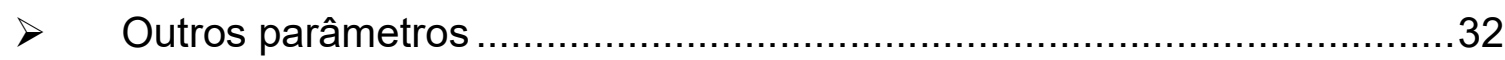

2.3. Justificativas para o envelhecimento das baterias ….................................35

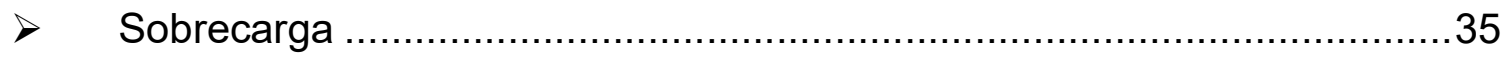

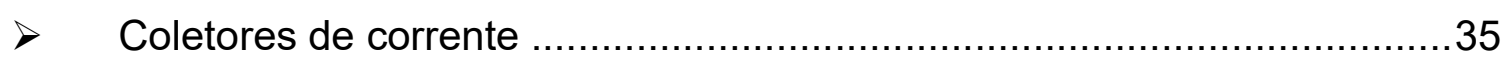

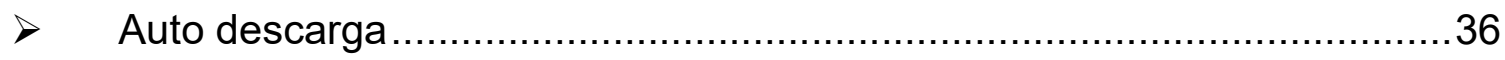

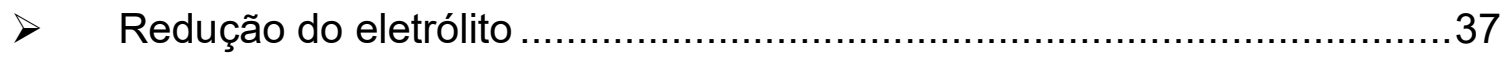

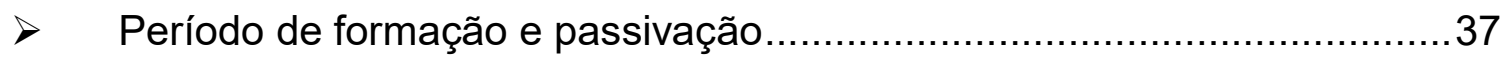

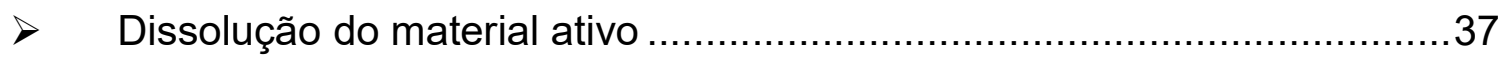

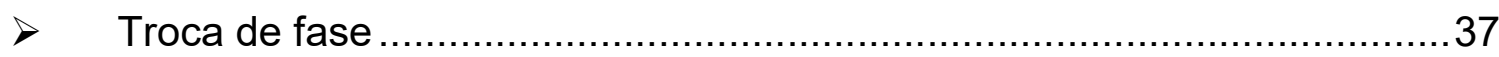

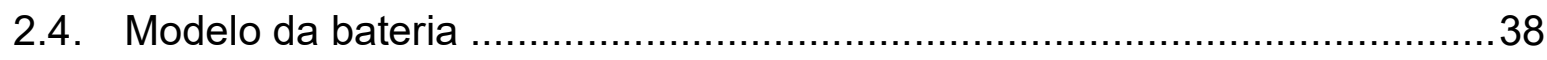


2.5. Exemplo de circuito de proteção .39

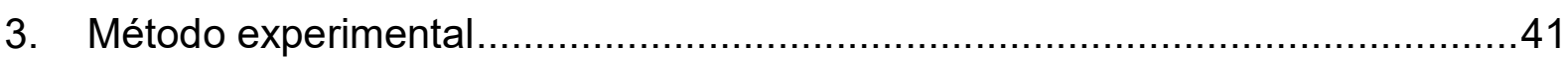

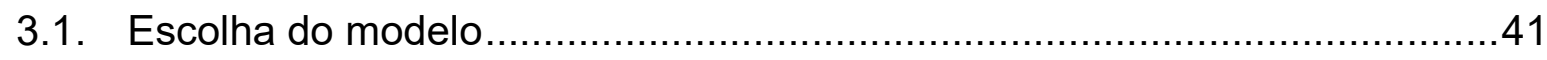

3.2. Cálculos de resistência e capacitância usando o ADC do microcontrolador 41

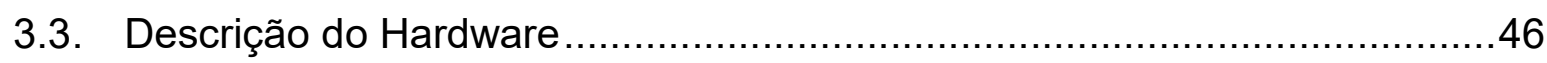

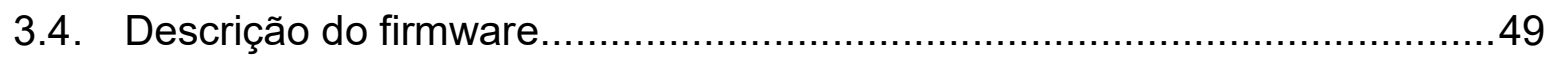

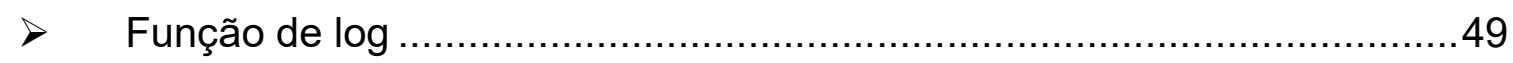

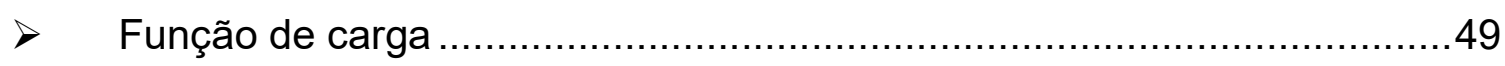

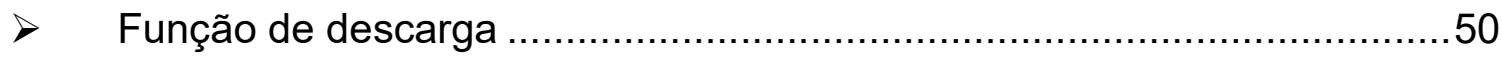

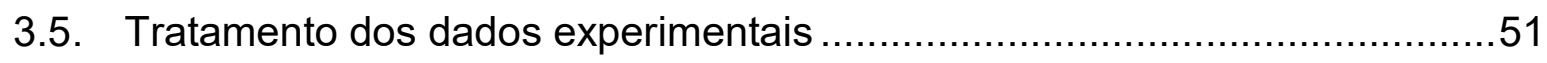

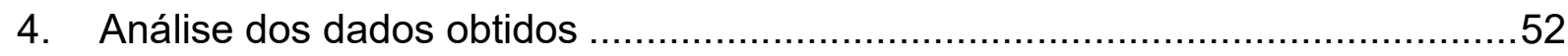

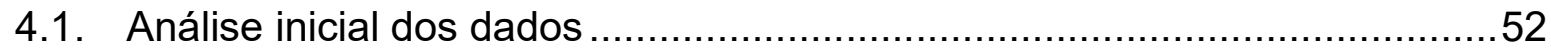

4.2. Problemas e imperfeições na medição …………………………….......56

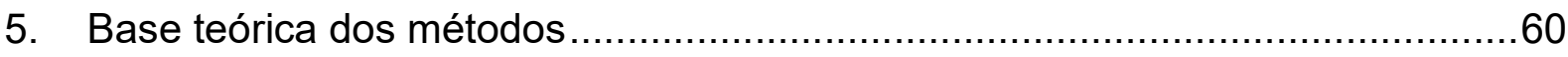

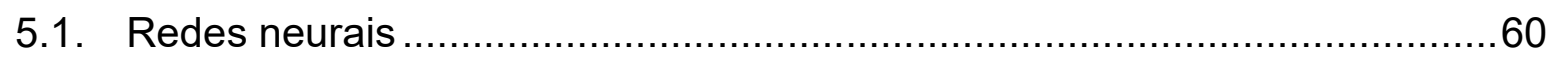

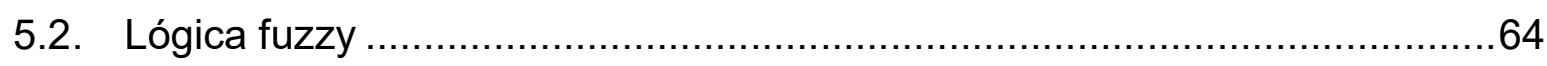

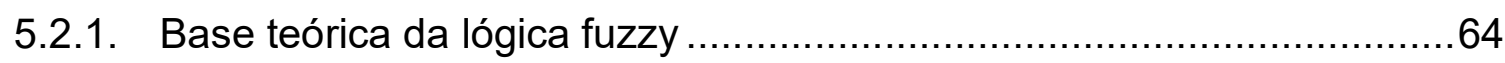

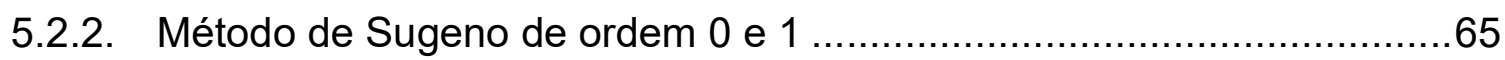

5.2.3. Exemplos de análise de baterias usando lógica fuzzy .........................68

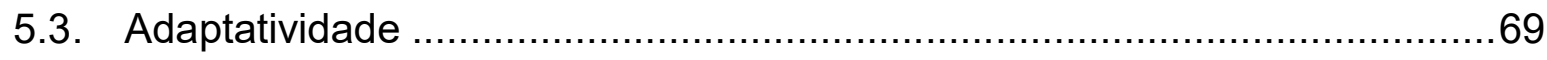

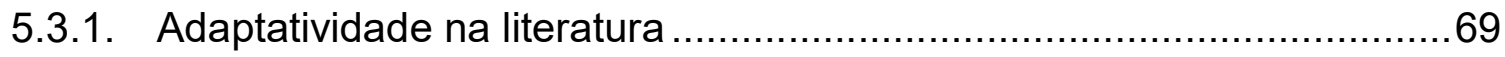

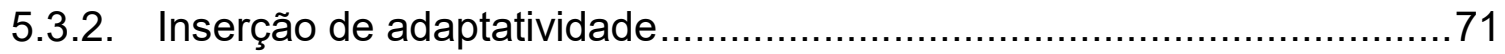

5.4. Melhorando a previsão de EOL com redes neurais …...............................73

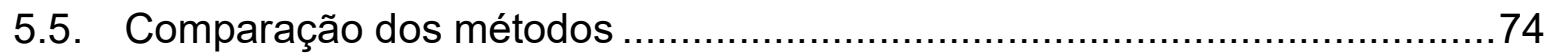

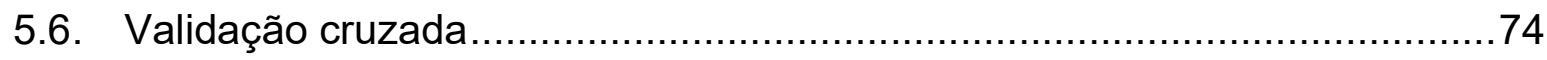

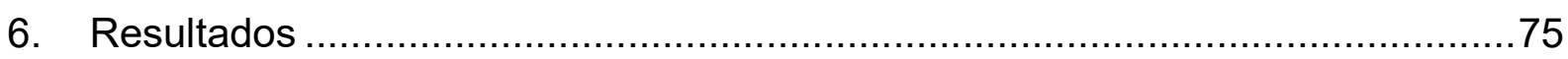

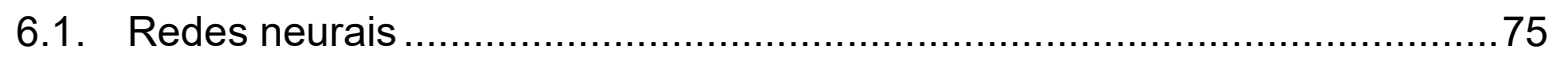




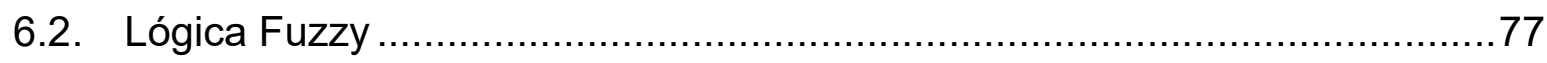

6.2.1. Resultados para o modelo de Sugeno de ordem 0 .............................77

6.2.2. Resultados para o modelo de Sugeno de ordem 1 1............................79

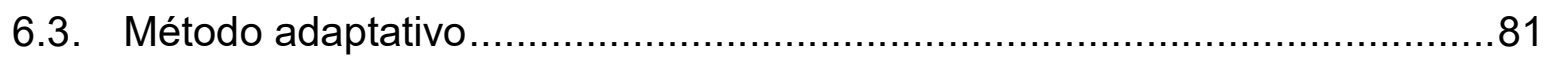

6.3.1. Primeira versão do algoritmo adaptativo ............................................. 81

6.3.2. Resultados da versão final do algoritmo adaptativo ..............................85

6.4. Melhoramento das previsões da rede neurais ............................................89

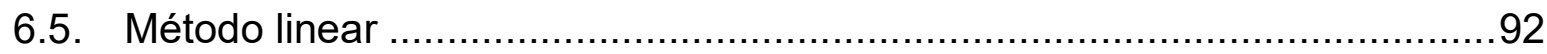

6.5.1. Resultados considerando somente a resistência.................................92

6.5.2. Resultados considerando somente a capacitância................................94

6.5.3. Resultados considerando a resistência e a capacitância .......................95

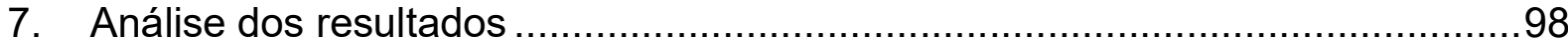

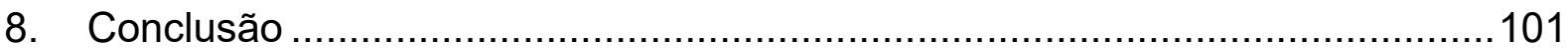

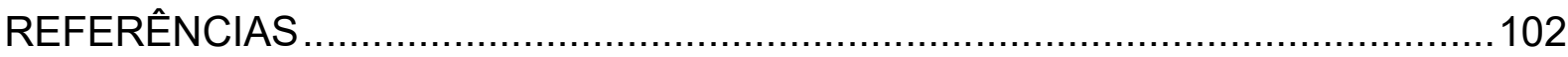

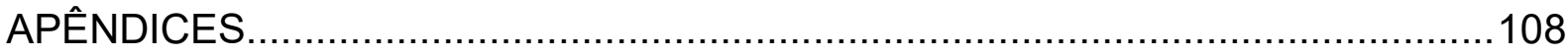

APÊNDICE A - Funções do FW de aquisição de dados....................................108

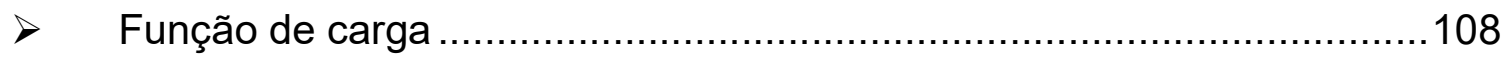

> Função de descarga nos primeiros 400ms ..........................................109

$>\quad$ Função de descarga .......................................................................110

APÊNDICE B - Extratos do SW criado para análise com rede neural .................111

APÊNDICE C - Extratos do SW criado para análise com lógica fuzzy ...............113

$>\quad$ Função de pertinência triangular.......................................................113

$>\quad$ Função de pertinência gaussiana .......................................................113

$>\quad$ Cálculo das funções de pertinência ......................................................114

$>\quad$ Cálculo do SOH estimado para o Sugeno de ordem 0 ……..................114

APÊNDICE D - Extratos do SW criado para análise com algoritmo adaptativo...117 APÊNDICE E - Extratos do SW criado para análise usando método linear.........120 
APÊNDICE F - Pesos da rede neural 9x5.

APÊNDICE G - Tabela de regras obtidas para o modelo de Sugeno de ordem 0 124

APÊNDICE H - Tabela de regras obtidas para o modelo de Sugeno de ordem 1 125

APÊNDICE I - Pesos obtidos para a segunda rede neural do método de melhoramento de EOL 128

APÊNDICE J - Pesos obtidos para a terceira rede neural do método de melhoramento de EOL 131

APÊNDICE K - Relação dos modelos de baterias utilizados 133

APÊNDICE L -Relação dos dados obtidos. 134

ANEXOS 145

ANEXO A - Extratos do datasheet da bateria Expower (PN: 483757) de 1000mAH 145

ANEXO B - Extratos do datasheet da bateria Expower (PN: 483759) de 1000mAH 147

ANEXO C - Extratos do datasheet da bateria HCT (PN: HCT053759P) de 1150mAH 148

ANEXO D - Extratos do datasheet do LPC1778 150

ANEXO E - Extratos do datasheet do FS312F-P. .154 


\section{Introdução}

\subsection{Objetivo}

O objetivo principal da pesquisa é analisar métodos com baixa complexidade computacional, que possam ser usados em diversos soluções com microcontroladores embarcados, que estimem a capacidade de baterias estacionárias de íons de lítio, que garantem o funcionamento de aplicações críticas em segurança. Para essa finalidade uma rede neural foi treinada e testada usando os dados recolhidos por um microcontrolador. Uma análise usando lógica fuzzy e adaptatividade também foi feita, podendo assim comparar os resultados entre as diferentes técnicas. Assim o microcontrolador conseguiria prever a capacidade da bateria e estimar a taxa de envelhecimento podendo indicar quando é necessário realizar a manutenção.

\subsection{Relevância das baterias estacionárias}

Baterias de íons de lítio são essenciais para diversos projetos em eletrônica e o seu uso se popularizou na primeira década deste século. Em 2008, a revista The Economist (2008) já indicava que estas baterias representavam $70 \%$ do mercado para celulares. Brodd (2001) mostrava que o crescimento das baterias de lítio foi exponencial durante essa década com o uso delas nesse mercado como mostra a figura 1:

Figura 1 - Crescimento do mercado de baterias de íons de lítio.

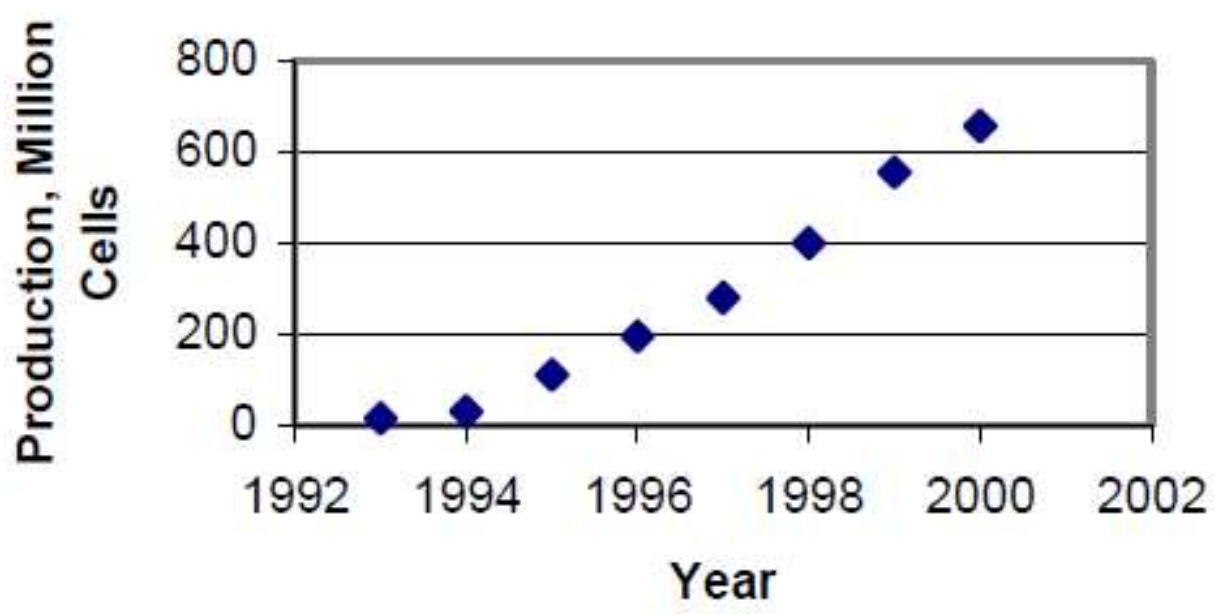

Fonte: Brodd (2001) 
Taiyou Research (2013) previu que o mercado de bateria recarregáveis cresceu de 10 bilhões de dólares em 2010 para cerca de 50 bilhões de dólares em 2020 graças ao crescimento dos veículos elétricos e dos sistemas de armazenamento de energia (usina eólicas por exemplo). A figura 2 mostra o crescimento do valor de mercado das baterias de lítio:

Figura 2 - Crescimento do mercado de baterias de lítio.

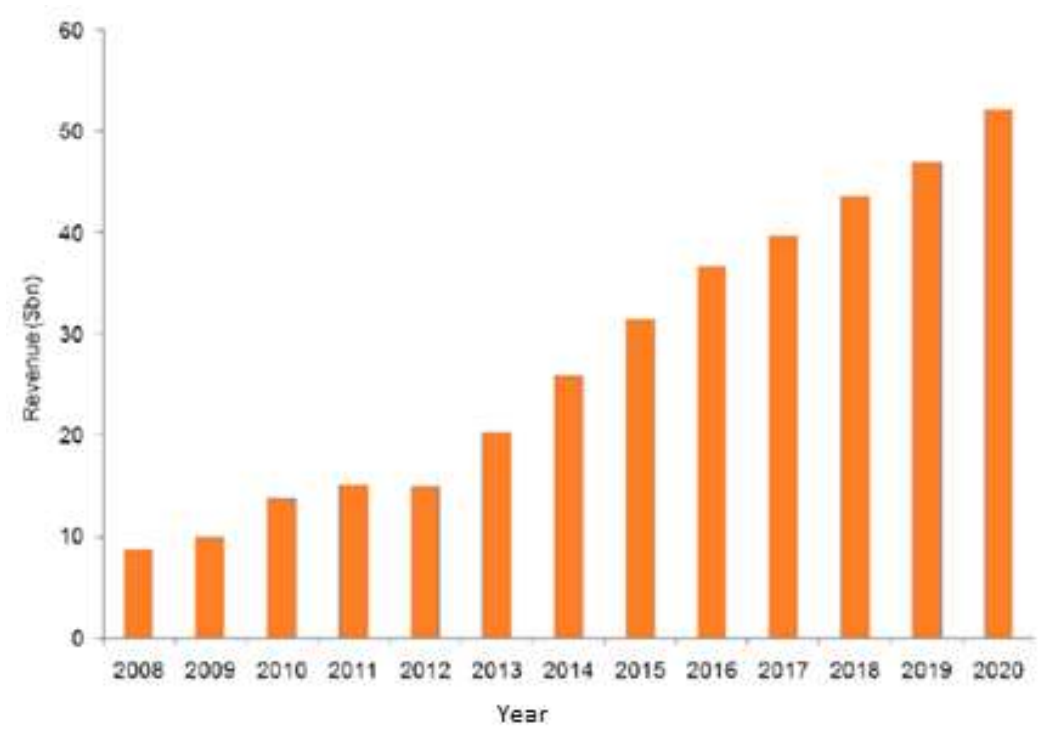

Fonte: Taiyou Research (2013).

Além dessa previsão de crescimento, a bateria de íon de lítio é o tipo de bateria mais importante no mercado atualmente como mostra a figura 3 :

Figura 3 - Mercado de baterias.

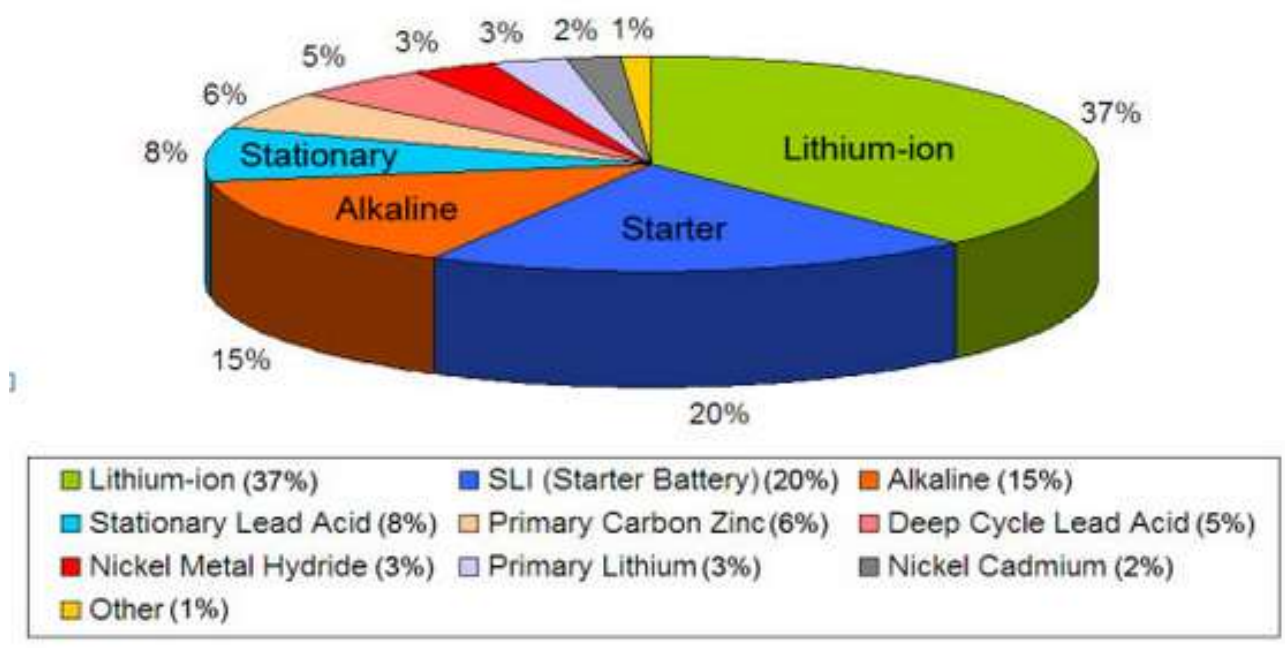

Fonte: Buchmann (2015). 
Para Taiyou Research (2013), o setor de eletrônicos de consumo é o que mais usa baterias de íons de lítio. Porém é possível encontrar esse tipo de bateria em diversas outras indústrias como, por exemplo, a indústria automobilística com os HEVs (Hybrid Eletric Vehicles) e EVs (Eletric Vehicles). Inclusive Chen et al (2013) argumenta que "as baterias de lítio são a única solução viável" para esse tipo de veículo. Passerini e Scrosati (2016) lembram que sistemas de armazenamento de energia sustentável como usinas de energia solar ou eólica dependem dessas baterias porque a produção da energia é irregular durante o tempo.

Essa é uma das aplicações de baterias estacionárias, que fornecem energia quando a fonte principal não está presente, ou seja, elas funcionam como uma reserva (buffer) para a fonte principal. Baterias estacionárias são também importante para sistemas de segurança se protegerem de quedas de energia. Buchmann (2015) cita entre outros exemplos, a bateria do carro que aciona o sistema de ignição, baterias em sistemas de produção de energia por energia solar e baterias de emergência em automação residencial. Este projeto focará em baterias de íon de lítio sendo usadas como baterias estacionárias em sistemas de segurança. Nesse tipo de aplicação ela só é utilizada em situações de emergência e presume-se que a bateria fique sempre carregada, sendo testada apenas em eventuais ciclos de manutenção preventiva.

Yanga et al (2017) explicam que essas baterias são usadas como estacionárias graças a maior densidade energética e o maior ciclo de vida. Para Choi e Aurbach (2016), densidade energética é a principal propriedade de uma bateria recarregável. Segundo Armand e Tarascon (2008), a densidade de uma bateria de íons de lítio é de até $180 \mathrm{Wh} / \mathrm{kg}$, ou seja, 5 vezes mais que as de chumbo ácido. Essa comparação é feita também por Buchmann (2015) e Linden e Reddy (2015) nas figuras 4 e 5 : 
Figura 4 - Comparação da densidade energética entre tecnologias.

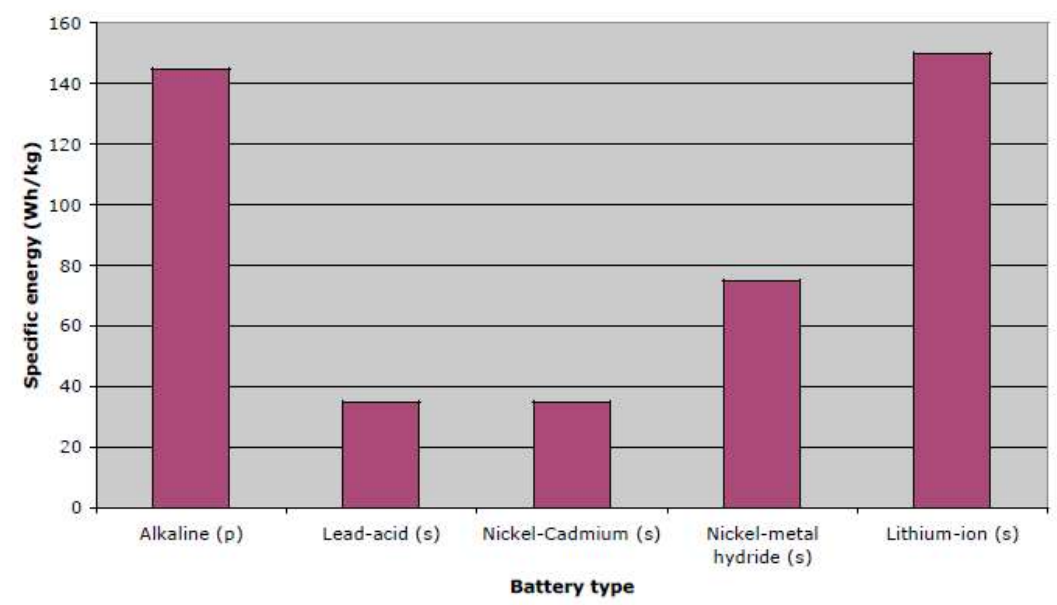

Fonte: Linden e Reddy (2015)

Figura 5 - Comparação da densidade energética entre tecnologias.

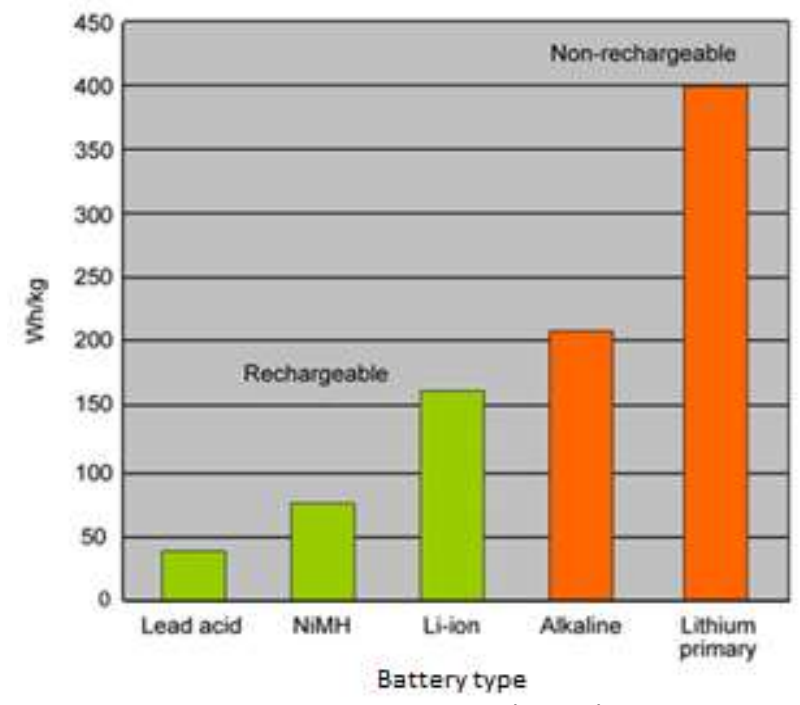

Fonte: Buchmann (2015).

As baterias de íons de lítio são mais caras que as outras baterias, porém para Buchmann (2015) as baterias de íons de lítio têm um custo por ciclo menor já que possuem baixa necessidade de manutenção e maior número de ciclo durante a sua vida útil. Esse maior número de ciclos é mostrado na figura 6 : 
Figura 6 - Comparação do número de ciclos entre tecnologias.

\section{Typical cycle life}

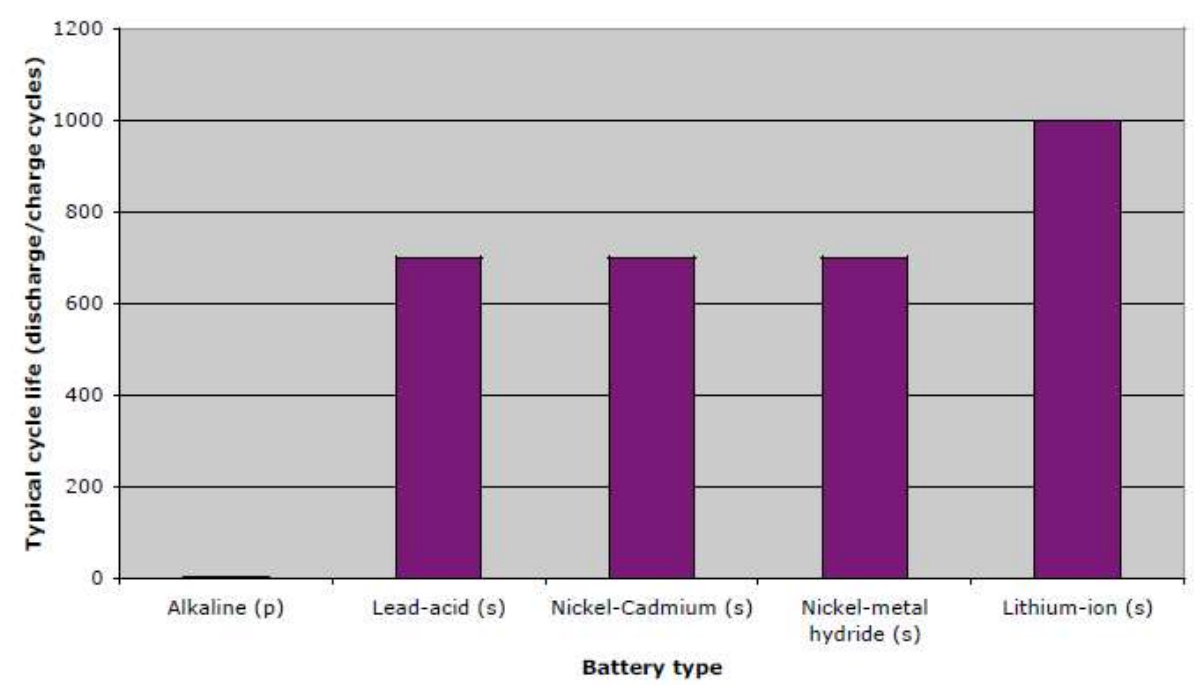

Fonte: Linden e Reddy (2015).

Além disso, o custo da bateria de íons de lítio vem caindo ano a ano como mostra a figura 7:

Figura 7 - Evolução do preço em $\cup \$ \$ / W h$ de diferentes formatos.

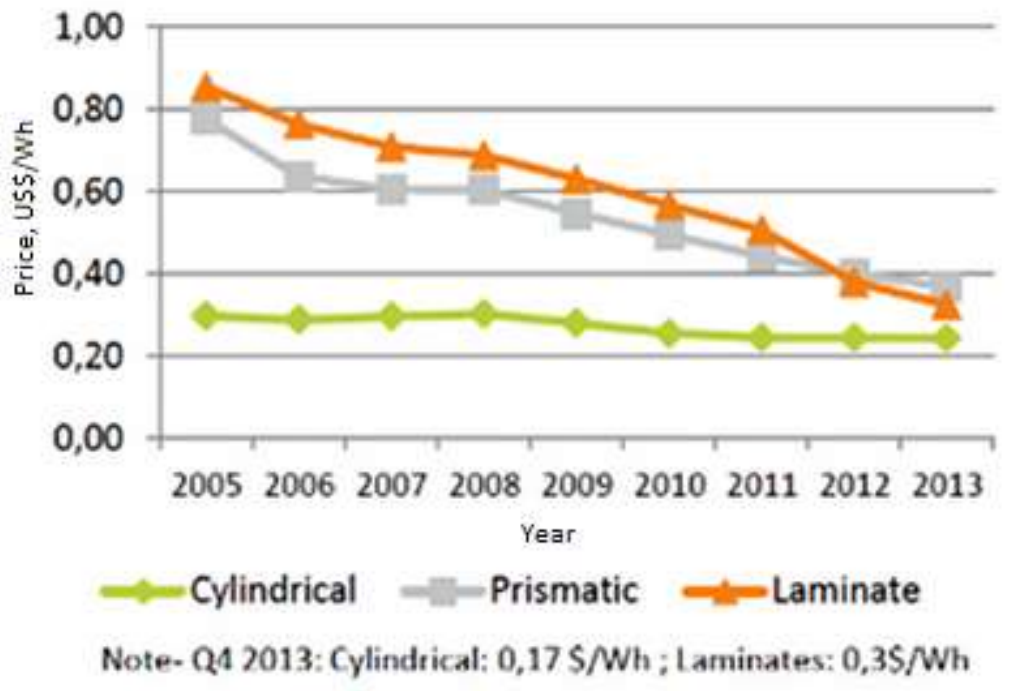

Fonte: Buchmann (2015)

Outra vantagem desse tipo de bateria de acordo com Armand e Tarascon (2008), é que "o mesmo íon ( $\left.\mathrm{LI}^{+}\right)$participa das reações nos dois eletrodos". Isso permite que a bateria seja mais simples e mais leve. 
Esses avanços tecnológicos são importantes também por oferecerem uma solução à combustíveis fósseis. Armand e Tarascon (2008) defendem que para substituir os combustíveis fósseis, "as baterias de íons de lítio deverão diminuir a sua pegada de carbono". Wu, Zhang e Chen (2016) também indicam que o fato de ser menos poluente é uma das razões da popularidade das baterias de lítio.

Portanto as baterias de íons de lítio fazem parte de um mercado em expansão e possuem qualidades e vantagens que justificam essa expansão. Os avanços nessa área permitem que esse tipo de bateria seja cada vez mais usado e por isso é importante e necessário o estudo dessas baterias para diversas aplicações, principalmente em aplicações de segurança onde as baterias estacionárias normalmente funcionam como garantia da não interrupção do sistema. Além disso, Choi e Aurbach (2016) dizem que a demanda por uma maior densidade energética continua alta e a dependência em baterias recarregáveis nos mercados de TI e transporte está aumentando, o que justificaria a intensa pesquisa no assunto. A figura 8 relata a importância dessa área com a quantidade de artigos sobre baterias de íons de lítio por país:

Figura 8 - Número de artigos sobre baterias de íons de lítio por país.

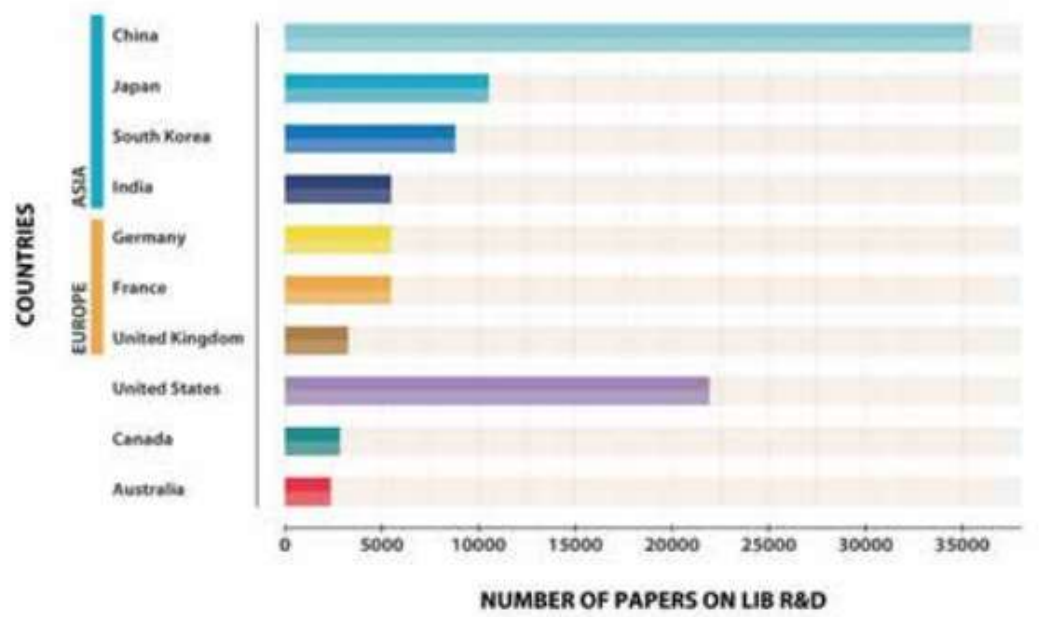

Fonte: Passerini e Scrosati (2016).

\subsection{Justificativas do projeto}

Além de garantir maior segurança para o usuário sobre o estado das baterias estacionárias, uma análise mais completa da qualidade e estado da bateria é imprescindível para evitar manutenção periódica desnecessária ou trocas. A medição do estado de vida de uma bateria está se tornando crucial já que um maior número de 
sistemas depende dessa fonte de energia (SBARUFATTI et al, 2017). Adicionalmente, com informações corretas sobre o estado da bateria, seria possível realizar a manutenção da bateria da maneira mais eficiente e com o menor custo além de gerar maior confiança na aplicação.

Portanto, a característica mais importante do projeto é a automação embarcada do processo de medição e, para tanto, é preciso que a solução tenha baixa complexidade computacional, ou seja, consiga ser executada com um microcontrolador sem que seja necessária mudança no projeto do sistema já embarcado. Assim seria possível utilizar a solução em um maior número de aplicações e com maior facilidade. Como argumentam Wu, Zhang e Chen (2016), as técnicas de gerenciamento de bateria estão precisando cada vez mais de hardware mais complexos. E Buchmann (2015) explica que apesar de muitas pesquisas usarem Espectroscopia de Impedância Eletroquímica (EIS - Electrochemical Impedance Spectroscopy), esse método envolve custo alto de equipamento, alto tempo de teste e eletrônica dedicada inviabilizando esse método em aplicações com ênfase em aplicações de segurança.

Por isso, os testes devem ser realizados com a bateria funcionando, pois, uma parte das aplicações de baterias estacionárias envolve circuitos de segurança e a bateria é a garantia da não interrupção do circuito. Uma forma de extrair os parâmetros em tempo real é necessária e é uma das principais preocupações dos artigos e trabalhos estudados (HE et al, 2011; JUANG, 2010; LE; TANG, 2011).

Para que o projeto apresente uma solução abrangente, é importante que a análise seja realizada apesar de mudanças na temperatura, ou no tempo de carga, ou na corrente de carga e descarga, ou em qualquer outro parâmetro que mude a capacidade de uma bateria. Esse objetivo é importante já que o usuário pode não ter controle sobre essas variáveis externas e como explicam Arora, White e Doyle (1998) e Dubarry et al (2017) essas variáveis mudam a capacidade da bateria. Por isso, a falta de um modelo matemático satisfatório que relaciona todas essas variáveis justifica o uso de inteligência artificial.

Wang et al (2016) lembram que o monitoramento da bateria é imprescindível para segurança do sistema já que a capacidade da bateria depende de tantos parâmetros internos e externos. É necessário que a solução funcione com fabricantes e modelos diferentes de bateria já que é possível que se tenha duas ou mais opções de bateria 
para a mesma aplicação. Como as baterias estão em constante evolução, é provável que a bateria de uma aplicação mude sem que o circuito mude. Portanto a solução não pode estar vinculada a um modelo de bateria. Assim a adaptatividade da solução é uma característica importante. Além disso, Dubarry et al (2017) lembram que materiais diferentes nos eletrodos fazem que as baterias de modelos diferentes envelheçam em ritmos diferentes. Além de apresentar soluções funcionais, é importante comparar as diferentes soluções usando rede neural, lógica fuzzy ou adaptabilidade, pois isso permite definir a solução ótima para determinada aplicação. Como as baterias de íons de lítio estão cada vez mais presentes no mercado e podem ser utilizadas em diversas aplicações, e elas fazem parte de diversos projetos, de baterias para celulares até HEV, há diversos estudos que tratam desse tema na literatura (COLEMAN; HURLEY; LEE, 2008; MUKHERJEE, 2003). Porém esses trabalhos normalmente não estabelecem um conjunto de parâmetros único já que a maioria foca em uma aplicação específica e no estudo de um determinado parâmetro de medição, não podendo ser comparados entre si. Isso resulta em uma falta de soluções para análise de baterias no mercado, como é mencionado por Xing et al (2011). 


\section{Modelos e parâmetros das baterias de íons de lítio}

\subsection{Descrição de uma bateria de lítio ("rocking chair")}

Existem diferentes tipos de baterias de íons de lítio e o que difere um tipo do outro é o material no catodo (BUCHMANN, 2015). Os materiais mais comuns são Cobalto e Manganês para o catodo e grafite para o anodo. Há também baterias de lítio polímero que possuem um eletrólito em gel que são normalmente montadas em embalagens de plástico (pouch cells). As vantagens das baterias pouch cell são a maior flexibilidade do formato (uma célula pode ter espessura de $1 \mathrm{~mm}$ ), pois pode ser organizada em lâminas que não precisam ser comprimidas, e a sua maior segurança à sobrecarga. Segundo Taiyou Research (2013), essas razões justificam o seu maior uso em veículos elétricos. Porém Choi e Aurbach (2016) lembram que as baterias cilíndricas possuem uma densidade energética $20 \%$ maior que as baterias do tipo pouch cell. Os três formatos mais populares são apresentados na figura 9:

Figura 9 - Formatos de bateria: a - cilíndrico. b - prismático. c - laminado ou pouch.
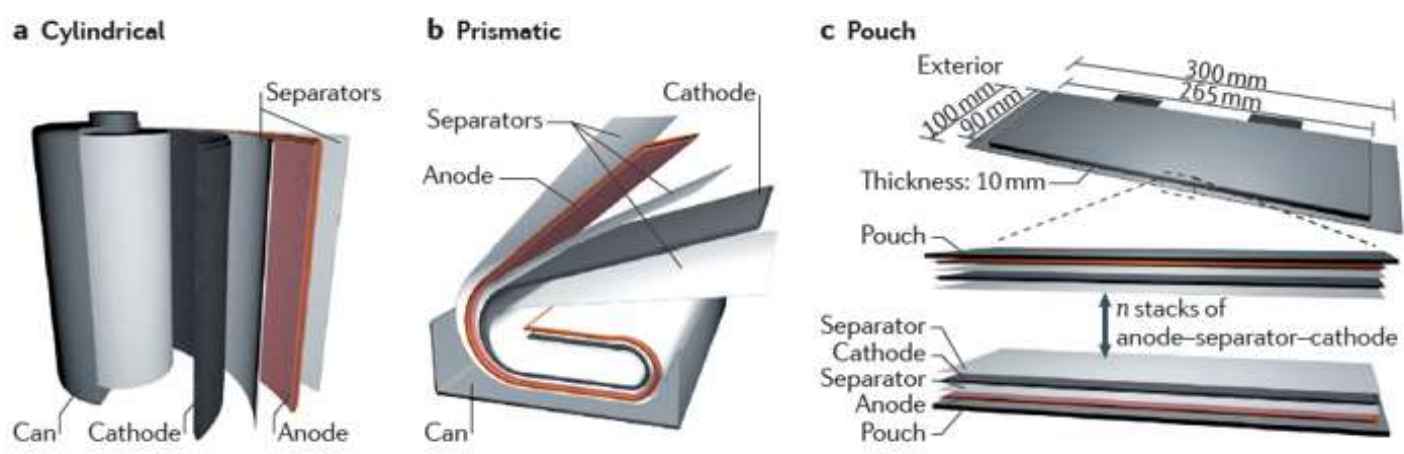

Fonte: Choi e Aurbach (2016).

O anodo é o eletrodo onde a reação de oxidação ocorre, liberando elétrons para o circuito e o catodo é o eletrodo onde a reação de redução ocorre, recebendo elétrons do circuito. Para uma bateria, o eletrodo positivo se comporta como catodo durante a descarga e anodo durante a carga. O contrário ocorre no eletrodo negativo. O padrão é chamar os eletrodos considerando a etapa de descarga, ou seja, o eletrodo positivo é o catodo e o negativo é o anodo.

Scorsati, Croce e Panero (2001) explicam que uma bateria de íons de lítio é produzida juntando materiais "pobres em lítio" que reagem com os íons de lítio no anodo (grafite é o mais comum $\mathrm{LiC}_{6}$ ) com materiais "ricos em lítio" no catodo (exemplo: oxido de lítio 
cobalto: $\mathrm{LiCoO}_{2}$ ). Além disso, o eletrólito é formado por uma solução com íons de lítio (exemplo: LiPF6). Essa troca de íons de lítio nas duas reações químicas (a de redução e a de oxidação) é uma vantagem desse tipo de bateria. Esse movimento de ida e volta dos íons de lítio ficou conhecido como o conceito da "rocking chair" (cadeira de balanço), onde os volumes de íons de lítio do catodo e anodo mudam durante a carga e a descarga e por isso a analogia com a cadeira de balanço (The Economist, 2008; BRODD, 2001).

ĺons de lítio são armazenados no anodo entre camadas de carbono criando um potencial eletroquímico baixo o suficiente para o material se comportar como eletrodo negativo (IDOTA et al, 1997). Buchmann (2015) mostra diferentes tipos de catodos, vale ressaltar os mais usados: o oxido de lítio cobalto que é organizado em camadas como mostra a figura 10:

Figura 10 - Estrutura do catodo de Lítio-Cobalto.

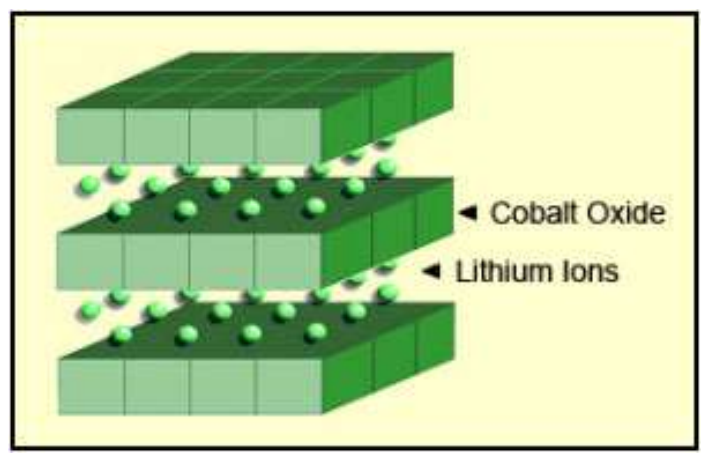

Fonte: Buchmann (2015).

Outro exemplo é o oxido de Lítio Manganês que é organizado em cristais tridimensionais como mostra a figura 11:

Figura 11 - Estrutura do catodo de Lítio-Manganês.

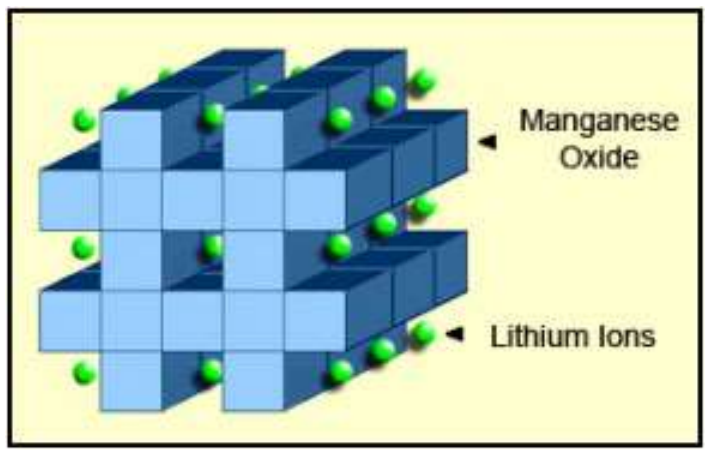

Fonte: Buchmann (2015) 
As equações (2.1 a 2.3) que descrevem as baterias de íons de lítio durante a carga são mostradas por Arora, White e Doyle (1998) e Gold Peak Industries Ltd (2003):

$$
\begin{gathered}
\text { Eletrodo positivo: } \mathrm{xLi}^{+}+\mathrm{x}^{-}+\mathrm{C}_{6} \rightarrow \mathrm{Li}_{x} \mathrm{C}_{6} \\
\text { Eletrodo negativo: } \mathrm{LiCoO}_{2} \rightarrow \mathrm{yLi}^{+}+\mathrm{ye}^{-}+\mathrm{Li}_{1-y} \mathrm{CoO}_{2} \\
\text { Geral: } \mathrm{LiCoO}_{2}+\mathrm{C}_{6} \rightarrow \mathrm{Li}_{x} \mathrm{C}_{6}+\mathrm{Li}_{1-x} \mathrm{CoO}_{2}
\end{gathered}
$$

Essas equações valem para as baterias de oxido de lítio cobalto $\left(\mathrm{LiCoO}_{2}\right)$. As equações para os outros tipos de bateria são análogas.

\subsection{Descrição dos parâmetros de medição da bateria}

A capacidade de uma bateria é medida em Amperes-horas (Ah) ou miliamperes-horas (mAh). Os fornecedores usam também a escala em $C$ para indicar a corrente que a bateria deve operar. 1C é a corrente que uma bateria precisa para carregar ou descarregar totalmente em uma hora. Por exemplo, uma bateria com capacidade de $1000 \mathrm{mAh}$ com uma carga de $0.25 \mathrm{C}$ (ou $250 \mathrm{~mA}$ ) deve funcionar por 4 horas com a sua capacidade nominal. Ou seja, a capacidade da bateria representa a quantidade de energia que ela possui. A resistência interna da bateria transforma parte da corrente em calor, por isso há uma pequena diferença entre o tempo de descarga prático e o teórico, quão maior é a corrente de descarga em relação a nominal, menor é o tempo de descarga prático (BUCHMANN, 2015). Esse processo é descrito na figura 12 que compara a capacidade atingida com as diferentes correntes de descarga:

Figura 12 - Capacidade em função da descarga.

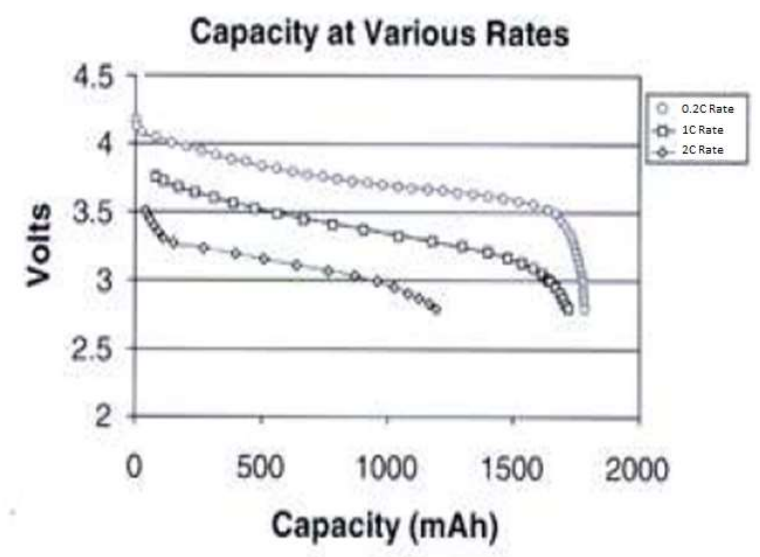

Fonte: Gold Peak Industries Ltd (2003). 
Por isso os fabricantes normalmente indicam qual a corrente de descarga padrão para obter a capacidade nominal. Juang (2010) explica que esse fenômeno é descrito pela lei de Peukert:

$$
K \times I^{n-1}=\text { contante } \quad, \mathrm{n}>1
$$

K é a capacidade da bateria em Ah, I é a corrente de descarga e $n$ depende das características da bateria. Com $n=1$ representa a bateria ideal que não muda de acordo com a corrente de descarga. Segundo Juang (2010), n varia entre 1,2 e 1,5 para baterias comerciais.

Dois parâmetros essenciais para a análise do estado da bateria são o SOC (State Of Charge ou estado da carga) e o SOH (State Of Health ou estado de saúde).

\section{SOC: State of Charge}

O SOC descreve o quanto a bateria já foi descarrega em relação a sua capacidade nominal. Como esse parâmetro é escrito em porcentagem, 100\% representa uma bateria com a carga igual a sua capacidade nominal e $0 \%$ representa uma bateria totalmente descarregada (LU et al, 2013).

Coleman, Hurley e Lee (2008) descrevem mais formalmente o SOC como a carga que resta na bateria descrita pela equação 2.5 .

$$
\operatorname{SOC}(t)=\operatorname{SOC}\left(t_{0}\right)-\frac{1}{A H C} \int_{t_{0}}^{t} i(\tau) d \tau
$$

$\mathrm{Na}$ equação AHC é a capacidade nominal em Ah. Portanto o SOC é dependente da corrente que já foi fornecida, por isso um dos métodos para prever o SOC é contagem de Coulomb (JUANG, 2010). Medindo a corrente de saída é possível prever o quanto resta de carga na bateria. Para Hu et al (2015) o método de Coulomb necessita de uma medição de corrente precisa e normalmente de um ciclo completo de carga e descarga. Os outros métodos são a estimativa pela tensão nos terminais ou pela impedância de saída. O problema de estimar o SOC pela tensão nos terminais é que a impedância da descarga ou da carga influencia na medição e muitas vezes não é possível medir a tensão em circuito aberto (JUANG, 2010). 
Xing et al (2011) enumeram os diferentes métodos para estimar o SOC, além da contagem de Coulomb é citado métodos que utilizam o filtro de Kalman, lógica fuzzy ou um modelo auto regressivo de médias móveis.

\section{SOH: State Of Health}

Porém o SOC é inadequado para mostrar o estado de vida da bateria, pois uma bateria carregada terá um SOC de 100\% independente se ela está envelhecida ou nova. Para isso serve o $\mathrm{SOH}$. $\mathrm{O} \mathrm{SOH}$ é definido como a relação entre a capacidade atual da bateria e a capacidade nominal da bateria (CHEN et al, 2013; LE; TANG, 2011).

$$
S O H=\frac{C_{\text {bat }}}{C_{\text {nominal }}} \times 100 \%
$$

Lu et al (2013) lembram que o SOH pode ser definido usando outros parâmetros da bateria como impedância, taxa de auto descarga ou densidade energética. Como o $\mathrm{SOH}$ é expresso em porcentagem, fica mais fácil comparar o estado de baterias com cargas nominais diferentes. Portanto o $\mathrm{SOH}$ quantifica o quanto a bateria envelheceu, tanto que Lu et al (2013) preferem chamar o parâmetro de SOL (State of Life ou estado de vida). Diferentes fontes (YANGA et al, 2017; LU et al, 2013; GOLD PEAK INDUSTRIES LTD, 2003; GROOT, 2012; XING et al, 2011; HE et al, 2011) explicam que a bateria atinge o fim da vida (EOL - End Of Life) quando o SOH chega entre 70 e $80 \%$. He et al (2011) e Yanga et al (2017) lembram que uma bateria com SOH menor que $80 \%$ tem ciclos de carga e descarga muito instáveis, pois a sua perda de capacidade tem um caráter exponencial como é visto na figura 13:

Figura 13 - Capacidade em função do número de ciclos.

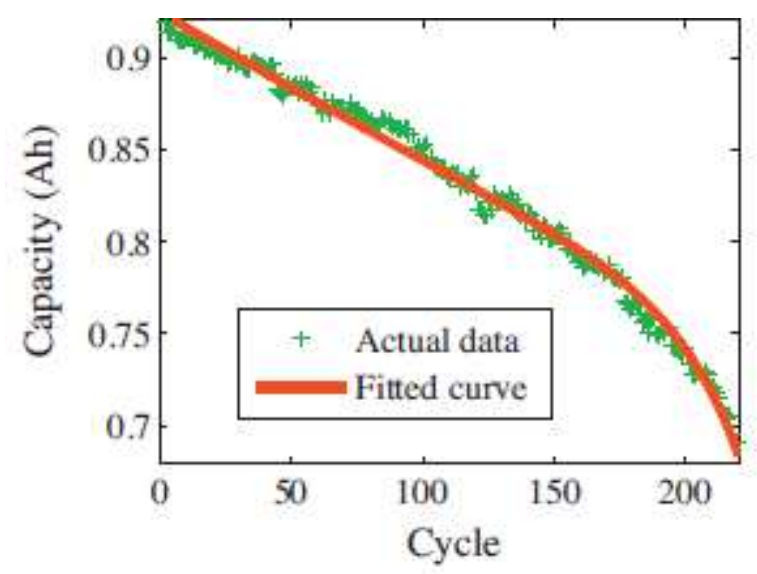

Fonte: He et al (2011). 
He et al (2011) e Chen e Pecht (2012) descrevem a perda da capacidade em função do tempo com a equação 2.7 .

$$
K=a e^{b t}+c e^{d t}
$$

$\mathrm{Na}$ equação $\mathrm{K}$ é a capacidade da bateria, $\mathrm{t}$ é o número de ciclos, a e b são relacionados à impedância interna e c e d são relacionados à taxa de envelhecimento (HE et al, 2011).

Xing et al (2011) relacionam três componentes comerciais que gerenciam baterias (Maxim DS2726, TI BQ78PL114 e O2 Micro OZ890) e nenhum deles faz o cálculo do SOH apesar de este parâmetro ser importante para definir quando é necessário trocar a bateria. Para Xing et al (2011), isso se deve pela dificuldade de medir o $\mathrm{SOH}$, pois o SOH varia de acordo com a temperatura e com a corrente de descarga como é visto nas figuras 14 e 15.

Figura 14 - Evolução do SOH em função da carga e da temperatura.

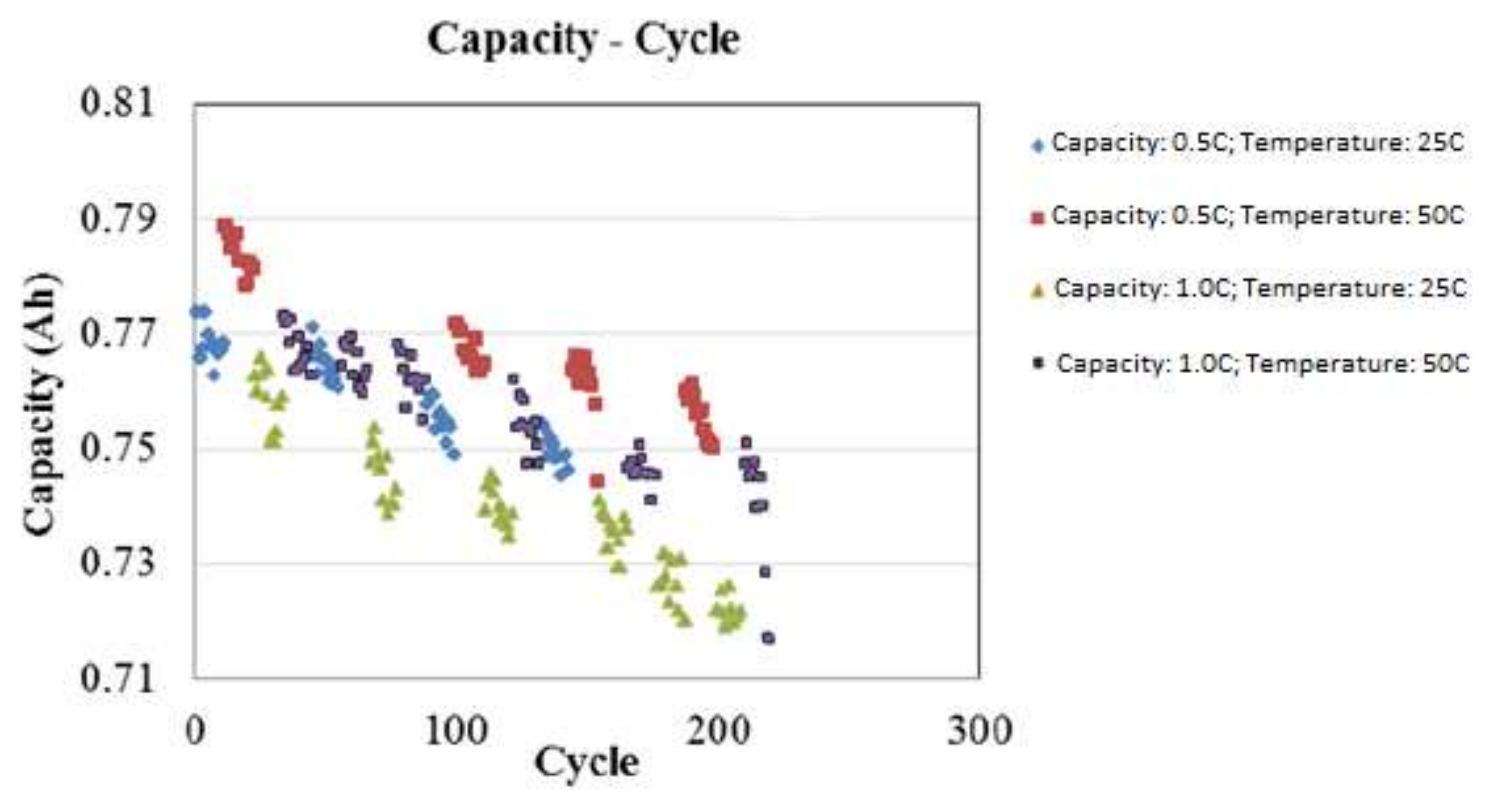

Fonte: Xing et al (2011).

Figura 15 - Evolução do SOH em função da carga. 


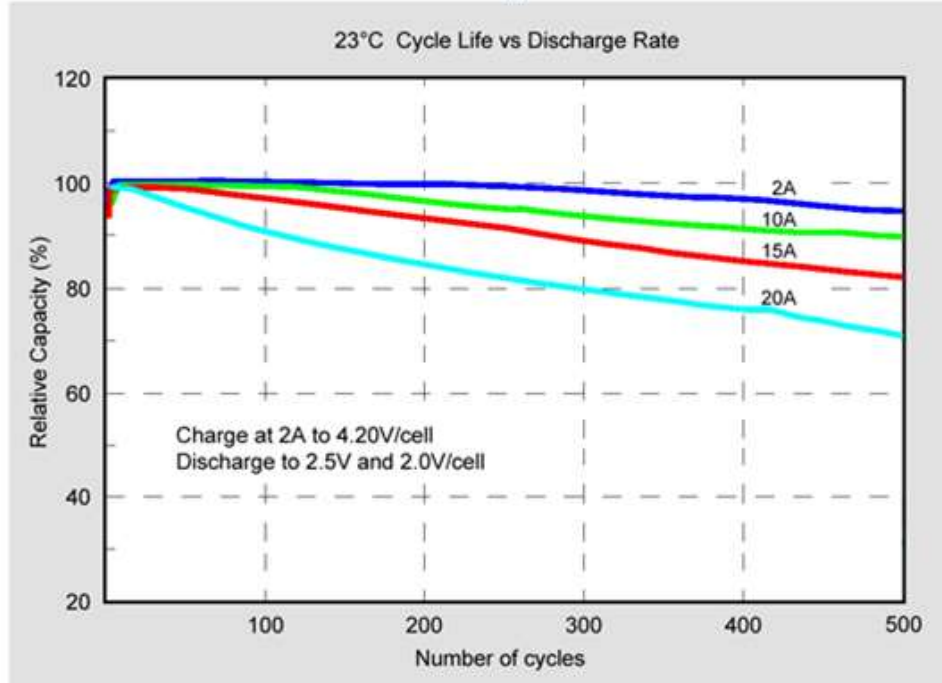

Fonte: Buchmann (2015).

Apesar disso, a Apple fornece nas versões posteriores ao IOS 11.3 informações sobre o SOH da bateria do Iphone (APPLE, 2019). Apesar da informação de como esse valor é calculado não ser divulgada, é possível inferir pelas informações descritas no site (APPLE, 2019) que o celular conta o número de ciclos de carga e descarga e compara com o uso em celulares parecidos. Além disso, a Apple (2019) descreve um ciclo de carga e descarga de uma forma diferente do que é comum na literatura e nos datasheets de baterias (anexos A, B e C). Para a Apple (2019), um ciclo completo é considerado quando a bateria for descarregada com uma quantidade equivalente à sua capacidade nominal independente se houve carga da bateria durante esse processo. Na literatura, um ciclo completo é considerado quando a bateria é descarregada completamente após uma carga completa. Como na maioria das aplicações não é possível obter o controle do processo e a quantidade de dados que a Apple possui das baterias usadas, a medição do $\mathrm{SOH}$ continua um desafio, principalmente em baterias estacionárias voltadas à segurança que normalmente não são descarregadas constantemente.

Além disso, Dubarry et al (2017) concluem que o SOH é dependente das condições de vida da bateria e que por isso cada bateria degrada de forma diferente, o que justifica uma análise profunda do $\mathrm{SOH}$ para um melhor gerenciamento da bateria, principalmente em aplicações de segurança que possuem condições particulares comparadas a outras aplicações. E Remmlingera et al (2011) argumentam que o 
conhecimento do $\mathrm{SOH}$ permite identificar uma degradação abrupta da bateria e é necessário para uma operação segura.

Portanto é complicado utilizar os estudos em laboratórios em uma solução comercial onde há variação na temperatura, na corrente de descarga e no modelo de bateria principalmente em casos onde essas informações não são conhecidas. Por isso Saha, Goebel e Christophersen (2009) argumentam que um sistema confiável de estimativa de SOC e SOH traria uma ajuda significativa a qualquer sistema envolvendo baterias.

\section{Outros parâmetros}

Outro conceito usado no estudo de baterias é o SOF (State of Function ou estado de funcionalidade). Para Lu et al (2013), o SOC mostra o quanto a bateria difere de uma bateria carregada e o $\mathrm{SOH}$ mostra o quanto uma bateria difere de uma bateria nova. Para Lu et al (2013) o SOF pode ser medido em porcentagem e indica a performance atual da bateria. Juang (2010) classifica o SOF como uma pergunta de Sim ou Não que indica se a bateria está apta a realizar determinada função de uma aplicação. 0 exemplo mais comum é se a bateria do carro tem carga suficiente para ligá-lo. O SOF depende do SOH e principalmente do SOC. Lu et al (2013) usam a figura 16 para explicar a relação entre esses parâmetros:

Figura 16 - Relação entre o SOC, o SOH e o SOF.

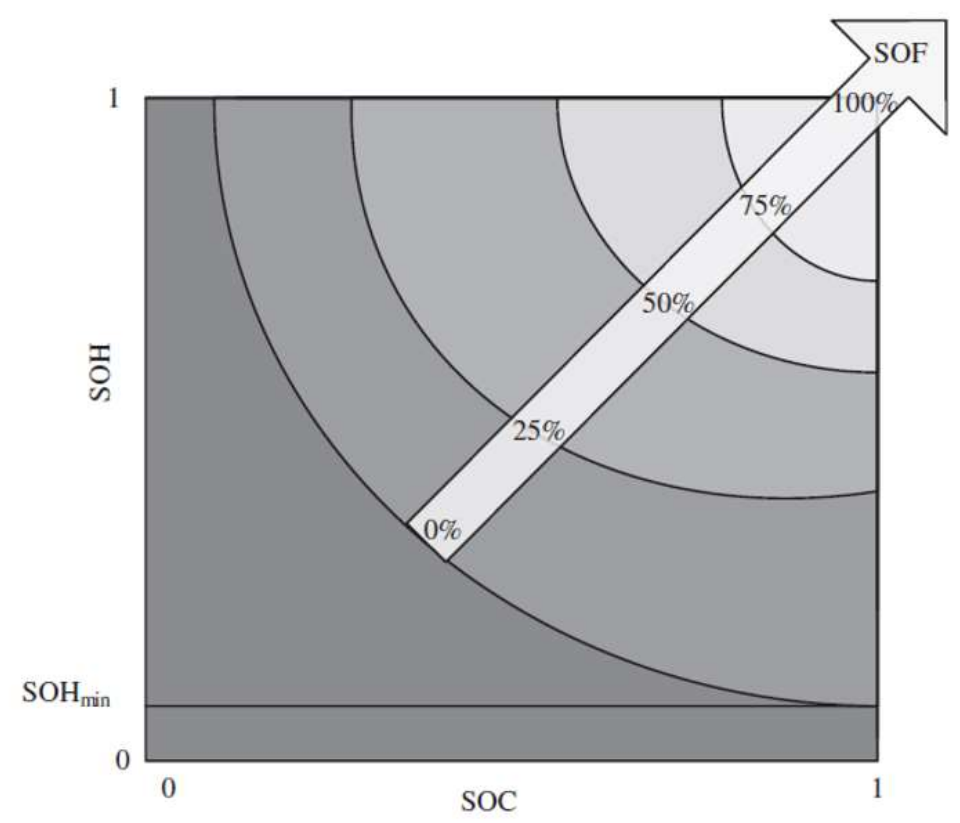

Fonte: Lu et al (2013). 
Na figura 16, o SOH mínimo representa o ponto de fim de vida (EOL) e as diferentes curvas representam os diferentes SOF, ou seja, os diferentes estados de performance da bateria. Portanto quanto mais velha a bateria fica (menor $\mathrm{SOH}$ ), menor a sua performance (SOF) para um mesmo estado de carga (SOC). Para Chiang, Sean e Ke (2011), o SOF é mais importante para o gerenciamento de baterias, principalmente em veículos elétricos, para evitar falhas durante a operação.

Os estudos do SOC e do SOH fazem parte de um estudo mais amplo: gerenciamento de prognósticos e saúde (Prognostics and health management ou PHM). Na literatura fala-se também do BMS (Battery Management System) que engloba o estudo do SOC, do SOH e do SOF. Lu et al (2013) argumentam que não há um consenso sobre a exata definição de BMS, já que o sistema pode envolver diversas soluções eletrônicas ou mecânicas e a bateria gerenciada pode ser de qualquer tipo ou ser um conjunto de baterias. He et al (2011) explicam que o PHM utiliza de métodos e tecnologias para garantir o bom funcionamento de um produto evitando falhas e riscos durante a sua vida útil, ou seja, o PHM utiliza sensores e modelos da bateria para garantir a qualidade da bateria e melhorar a manutenção (SBARUFATTI et al, 2017). Para He et al (2011), o PHM de uma bateria pode ser dividido em 2 métodos: física da falha (physics-of-failure ou PoF) e orientação por dados. Enquanto o método PoF é mais completo pois estima os problemas e riscos que a bateria pode sofrer com 0 conhecimento de sua física, o método por orientação por dados utiliza de estudos estatísticos de diferentes parâmetros da bateria para estimar possíveis falhas. Um dos objetivos desses métodos é prever a vida útil remanescente (remaining useful life ou RUL). O RUL é o tempo remanescente até um ponto de falha ou EOL considerando o sistema em que a bateria está inserida.

Como já foi explicado, a bateria atinge o EOL entre 70\% (XING et al, 2011) e 80\% (YANGA et al, 2017; CHEN; PECHT, 2012) de SOH dependendo da fonte e dependendo da aplicação (por exemplo, baterias de satélites têm um EOL menor porque são carregadas periodicamente). Juang (2010) lembra que esse ponto de corte é arbitrário e vários elementos da aplicação devem ser levados em conta ao determina-lo (como curva de corrente, temperatura e números de ciclos). O nome usado por Xing et al (2011) para o conceito do EOL é estado de vida (State of Life ou SOL). Para esse trabalho definiu-se o EOL em $72 \%$. Esse valor foi escolhido levando em conta os dados obtidos das baterias testadas, a aplicação dessas baterias em 
soluções de segurança e os comportamentos obtidos com cada método escolhido. É importante notar que para aplicações de segurança, a escolha do valor de EOL é crucial já que ao mesmo tempo garante o SOF da bateria para determinada aplicação e otimiza o momento de troca da bateria, reduzindo custo. Essa escolha, portanto, varia de caso a caso e não influi nos métodos utilizados mas deve ser projetada considerando que abaixo desse valor as baterias têm um comportamento instável em relação à sua capacidade.

Os autores Chen e Pecht (2012) e Saha, Goebel e Christophersen (2007, 2009) utilizam modelos parecidos para calcular o RUL. Eles introduzem um erro Gaussiano à equação de decaimento da capacidade e com teoria estatística Bayesiana, eles descrevem uma função de densidade probabilística (probability density function ou PDF) do RUL. Como o erro introduzido foi Gaussiano, a PDF será uma distribuição gaussiana (SAHA; GOEBEL; CHRISTOPHERSEN, 2009). Essa previsão é atualizada com os novos dados para compensar variações de cada caso usando o algoritmo de particle filtering (PF). He et al (2011) realizam algo parecido para prever a evolução do SOH e descrever a PDF do RUL, obtendo gráficos como o gráfico da figura 17.

Figura 17 - Previsão da evolução do SOH.

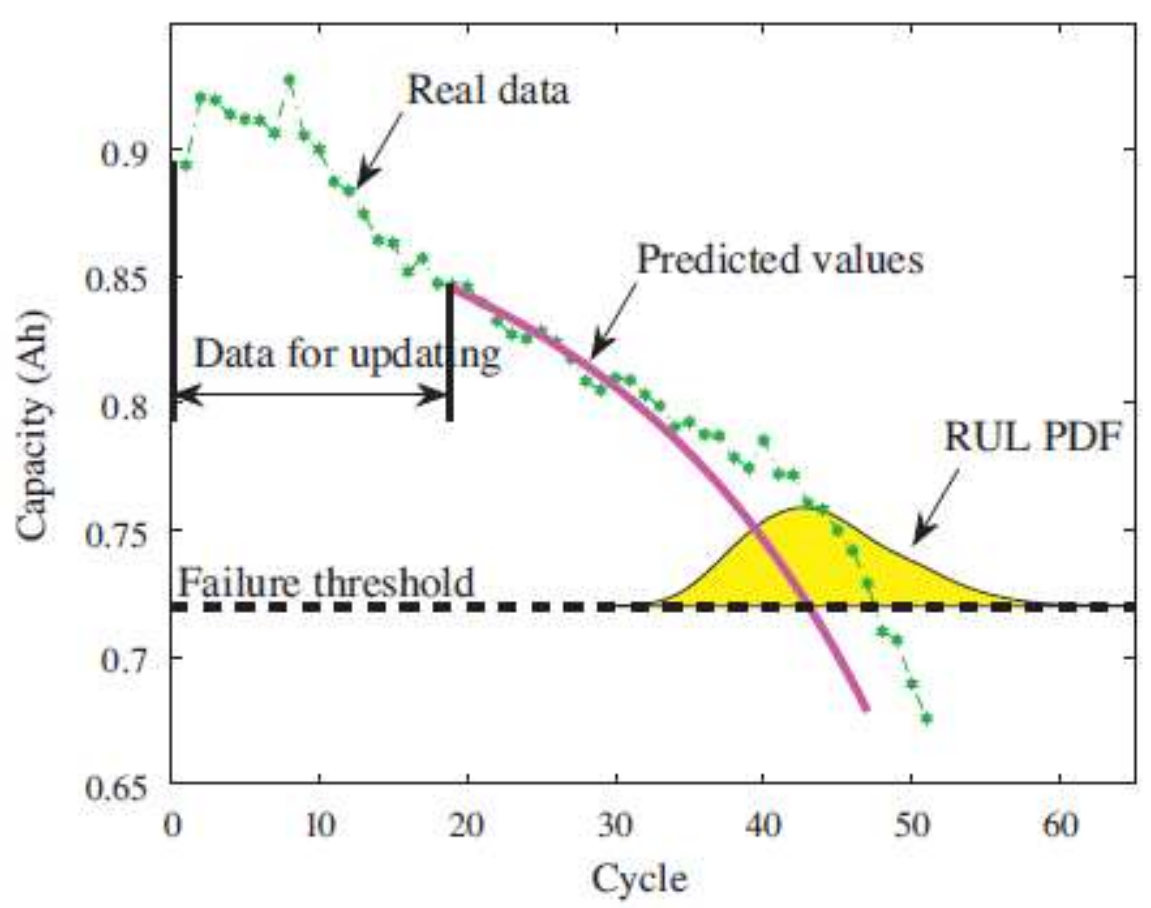

Fonte: He et al (2011). 


\subsection{Justificativas para o envelhecimento das baterias}

As baterias de íons de lítio devem funcionar em uma faixa de temperatura e tensão que depende do material usado na fabricação. Por exemplo, as baterias usadas nesse projeto podem ser descarregadas em temperaturas de -20 a $60^{\circ} \mathrm{C}$ e carregadas entre 0 e $45^{\circ} \mathrm{C}$ (ver anexos A, B e C). Segundo Lu et al (2013), se a bateria operar fora dessas faixas de temperatura e tensão, a performance e a segurança da bateria são comprometidas.

Arora, White e Doyle (1998) descrevem detalhadamente os diferentes processos eletroquímicos que podem causar perda da capacidade da bateria, ou seja, envelhecimento da bateria:

\section{Sobrecarga}

É possível ter sobrecarga nos eletrodos positivo e negativo e no processo de oxidação do eletrólito (ARORA, WHITE E DOYLE, 1998). Além de problemas na perda de capacidade da bateria, a sobrecarga pode criar sérios problemas de segurança com a liberação de gases inflamáveis dentro da bateria, estufando a bateria. Portanto a tensão máxima de carga influi na evolução do $\mathrm{SOH}$ como mostra a figura 18.

Figura 18 - Evolução do SOH em função da tensão máxima de carga.

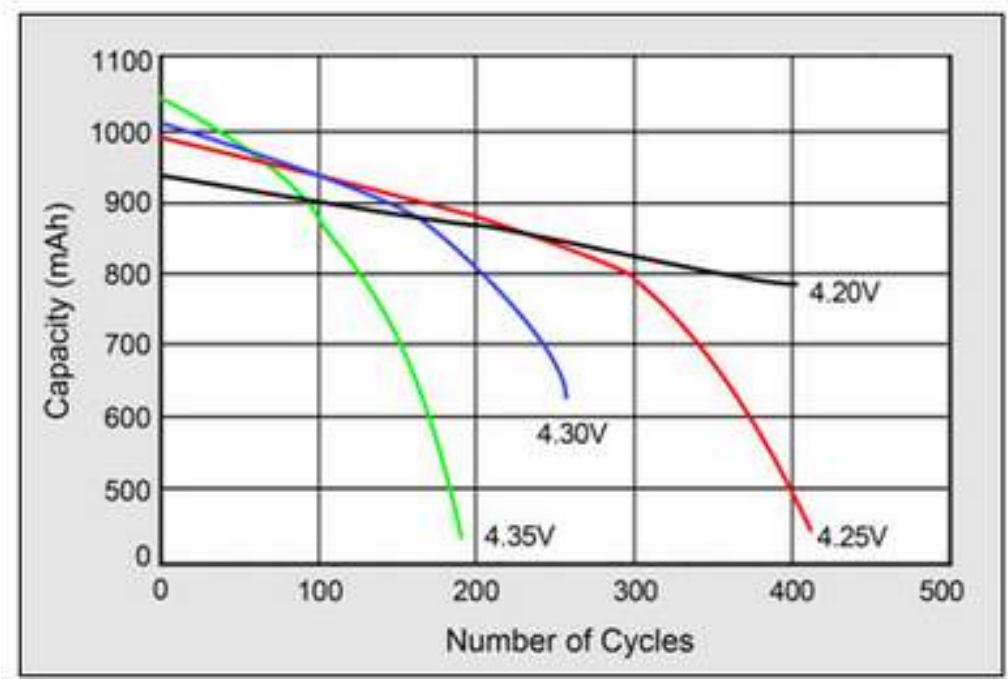

Fonte: Buchmann (2015)

\section{Coletores de corrente}

Cobre (no eletrodo negativo) e alumínio (no eletrodo positivo) são os mais usados coletores de corrente (ARORA, WHITE E DOYLE, 1998). E se esses metais sofrerem 
corrosão, a resistência interna da bateria aumentará diminuindo a capacidade da bateria.

\section{Auto descarga}

Esse processo acontece em todos os tipos de bateria e ele é mais crítico em outros tipos de bateria (BUCHMANN, 2015). Se a bateria não for carregada nem descarregada, ela perderá um pouco de carga ao longo do tempo. Auto descargas longas ou repetidas podem desregular a razão entre as massas de lítio ativas em cada eletrodo afetando a carga da bateria e consequentemente a sua capacidade máxima (ARORA, WHITE E DOYLE, 1998). A auto descarga aumenta com a temperatura e com a tensão da bateria, por isso as baterias não podem ser armazenadas em temperaturas acima de $60^{\circ} \mathrm{C}$ (GOLD PEAK INDUSTRIES LTD, 2003). Elas também devem ser armazenadas com $50 \%$ a $70 \%$ da carga. Em certas aplicações a bateria não é carregada até a carga máxima para prolongar o tempo de vida, mantendo a bateria com uma fração da capacidade máxima. A tensão de carga máxima para uma vida máxima da bateria de íons de lítio é de $3,92 \mathrm{~V}$, porém isso diminui a capacidade da bateria em até $60 \%$ (BUCHMANN, 2015). Além disso, os fabricantes vendem as baterias com $40 \%$ a $60 \%$ da carga total para diminuir o efeito da auto descarga enquanto a bateria não for usada. A auto descarga é de cerca de $5 \%$ nas primeiras $24 \mathrm{hs}$ e de 1 a $2 \%$ por mês (BUCHMANN, 2015). Portanto o aumento da temperatura ou o armazenamento em alto SOC envelhecem mais a bateria como pode ser observado na figura 19.

Figura 19 - Evolução da Capacidade em função da temperatura e SOC.

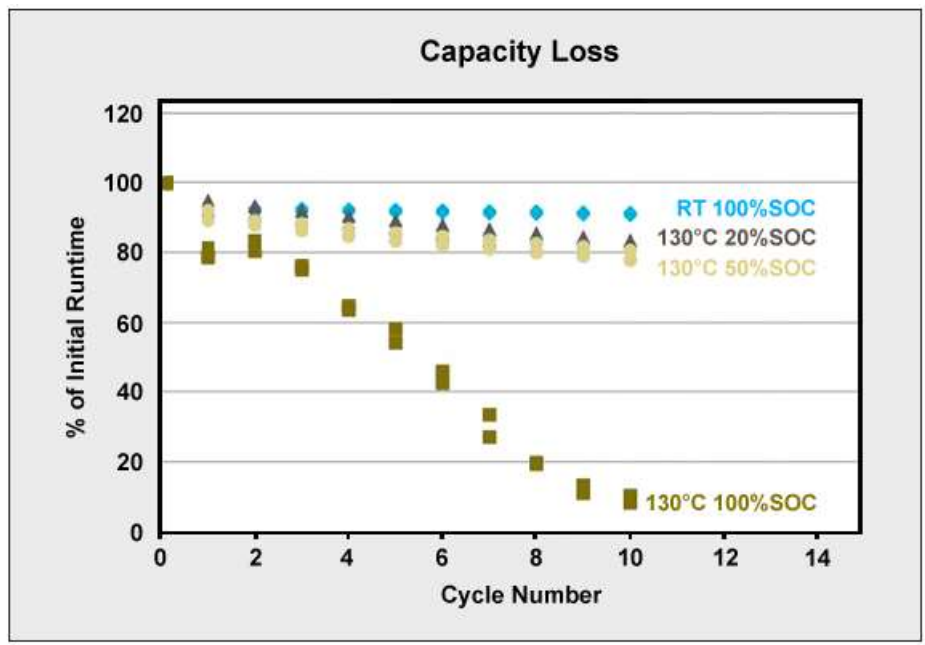

Fonte: Buchmann (2015). 


\section{Redução do eletrólito}

Arora, White e Doyle (1998) explicam que a redução do eletrólito é um processo esperado em células com eletrodo com carbono como base. Além de poder causar perda de capacidade da bateria, a redução do eletrólito pode causar liberação de gases em temperaturas mais altas reduzindo a temperatura máxima de segurança.

\section{Período de formação e passivação}

Nos primeiros ciclos de carga e descarga ocorre o processo de passivação do eletrodo de carbono reduzindo a massa de lítio ativa nesse eletrodo, desestabilizando assim a relação entre massas de lítio ativas em cada eletrodo (ARORA, WHITE E DOYLE, 1998), que diminuiu a capacidade da bateria. Por isso os fabricantes costumam utilizar um pouco mais de lítio no catodo do que o cálculo teórico para compensar essa desestabilização.

A passivação cria também uma perda da capacidade das baterias nos primeiros ciclos de carga e descarga (ARORA, WHITE E DOYLE, 1998). A consequência disso é que os fabricantes precisam realizar alguns ciclos de carga e descarga em temperaturas definidas até que a capacidade da bateria se estabilize, esses ciclos são necessários para evitar o aumento da resistência interna da bateria (BUCHMANN, 2015).

\section{Dissolução do material ativo}

Quando o material do eletrodo positivo foi totalmente descarregado, pode ocorrer a dissolução do material (exemplo do manganês) diminuindo a quantidade de material ativo. Além de diminuir a capacidade da bateria, isso pode causar aumento da resistência interna (ARORA, WHITE E DOYLE, 1998).

\section{Troca de fase}

Segundo Arora, White e Doyle (1998), o processo de troca de fase é inevitável com a diminuição dos íons de lítio no eletrodo positivo. A estrutura do material muda de forma de acordo com a quantidade de íon de lítio e do material do catodo. O problema pode acontecer quando há sobrecarga ou descarga excessiva no eletrodo positivo, piorando a dissolução do material ativo. Se as baterias forem descarregadas com uma corrente maior que o especificado, elas envelhecem mais rápido como é visto na figura 20. 
Figura 20 - Evolução da Capacidade em função da corrente de descarga.

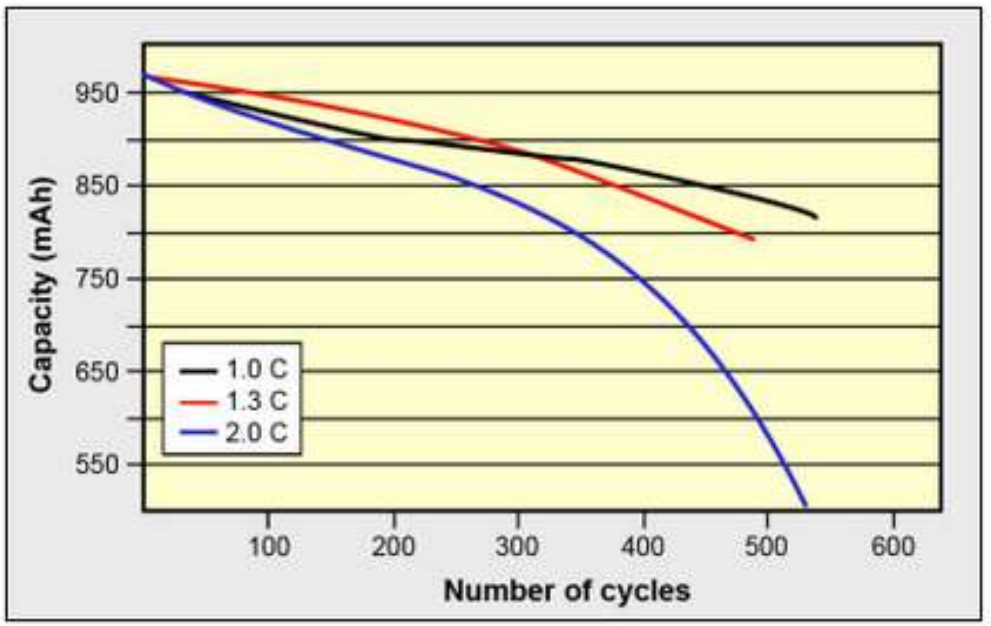

Fonte: Buchmann (2015)

\subsection{Modelo da bateria}

Diversas fontes (XING et al, 2011; SAHA; GOEBEL; CHRISTOPHERSEN, 2007, 2009) usam o modelo de Randle para modelar uma bateria de íons de lítio enquanto Juang (2010) usa o mesmo modelo para uma bateria de chumbo ácido. O modelo pode ser visto na figura 21 :

Figura 21 - Modelo de Randle de uma bateria.

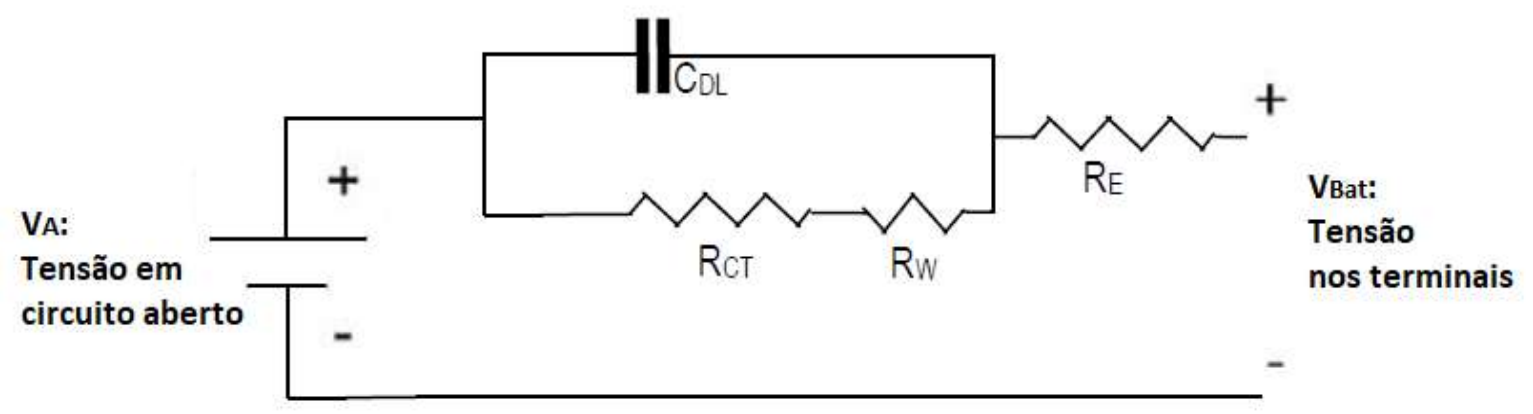

Fonte: Autor

Segundo Saha, Goebel e Christophersen (2009), os parâmetros do modelo são:

- A capacitância de dupla camada CDL

- A resistência de transferência de carga $R_{C T}$

- A resistência de Warburg Rw

- A resistência do eletrólito $R_{E}$.

Juang (2010) explica que a resistência RE é responsável pela resposta dinâmica a uma carga e que o capacitor $C_{D L}$ e as resistências $R_{W}$ e $R_{C T}$ representam as reações 
nos eletrodos. A resistência Rw e a capacitância CDL são normalmente ignoradas para estimar a perda de capacidade de uma bateria e há uma correlação entre a perda da capacidade e a resistência $\mathrm{R}_{\mathrm{CT}}+\mathrm{R}_{\mathrm{E}}$ (SAHA; GOEBEL; CHRISTOPHERSEN, 2007, 2009). Xing et al (2011) e Juang (2010) ignoram a resistência Rw ou incorporam em R $\mathrm{CT}$. Algumas causas do envelhecimento da bateria resultam em um aumento da resistência interna, por isso é possível estimar parâmetros da bateria com a resistência interna. Chiang, Sean e Ke (2011) citam outros modelos que utilizam circuitos RC em série ou composto somente de resistências. Porém Remmlingera et al (2011) argumentam que a resistência interna é mais dependente da temperatura do que da degradação da bateria, ou seja, não seria o método ideal se a temperatura for uma variável considerável.

\subsection{Exemplo de circuito de proteção}

Para garantir a segurança contra variações de temperatura e corrente, as baterias possuem circuitos de proteção. Buchmann (2015) descreve vários tipos de circuitos de proteção que podem ser usados em conjunto: fusíveis que abrem com uma corrente determinada, um circuito PTC (positive thermal coeficient) que aumenta a resistência interna com alta corrente, circuito CID (circuit interrupt device) que corta o circuito em caso de alta pressão na célula, chaves de estado solido que desconectam o circuito se necessário e um sistema de ventilação caso seja necessário liberar gás. O circuito também protege contra curto circuito dos terminais das baterias. Na foto da figura 22 é possível visualizar o circuito de proteção de uma bateria:

Figura 22 - Circuito de proteção em bateria de íons de lítio.

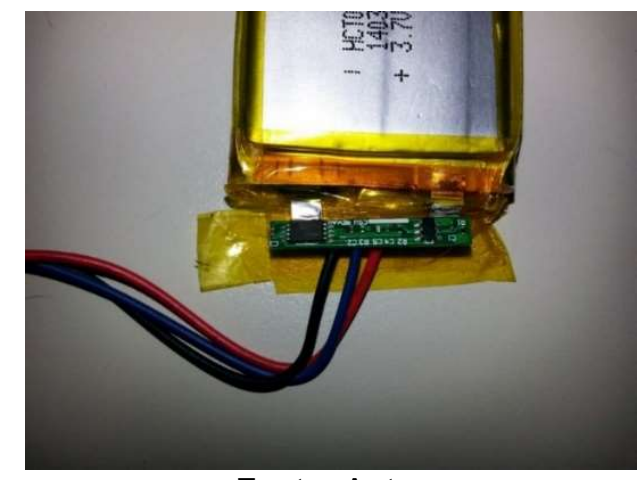

Fonte: Autor

O circuito de proteção dessa bateria possui dois chips, o FS312F-P e o FS8205A, ambos da empresa Fortune. O FD8205A é um chip que possui dois MOSFET canal N 
com o dreno curto circuitado. O FS312F-P que faz o controle da bateria, o circuito padrão está na figura 23.

Figura 23 - Circuito de proteção da bateria de íons de lítio.

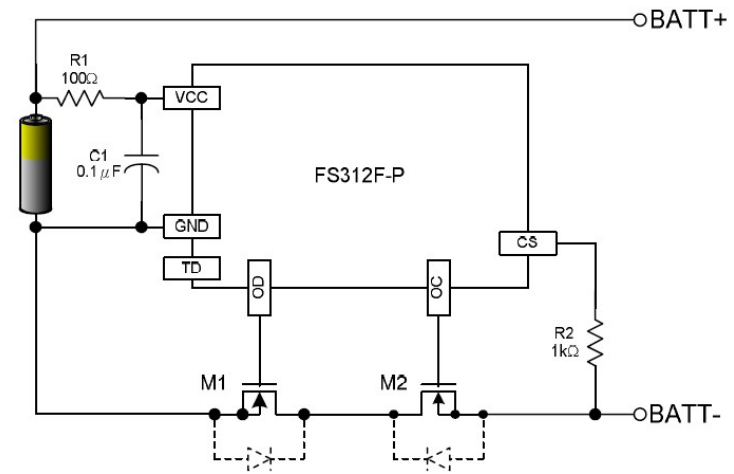

Fonte: Datasheet FS312F-P (Anexo E)

O chip FS312F-P controla a carga e a descarga da bateria acionando ou desligando os MOSFETs de carga e descarga. O pino CS detecta a carga da bateria e também uma sobre corrente. No datasheet que se encontra no anexo $\mathrm{E}$ há as diferentes situações que fazem o chip agir (por exemplo: tensão de sobre carga). Esse circuito contribui para a auto descarga da bateria, pois há uma corrente quiescente de $3 \mu \mathrm{A}$. 


\section{Método experimental}

\subsection{Escolha do modelo}

Para atender os objetivos do projeto, optou-se por utilizar o modelo da bateria de Randle sem diferenciar a resistência de transferência de carga $R_{c T}$ e a resistência de Warburg Rw. Como o objetivo é utilizar um firmware e hardware que possa ser incorporada à solução já embarcada, não foi possível fazer uma análise que possa diferenciar todos os componentes do modelo. Yanga et al (2017), Sbarufatti et al (2017) e Dubarry et al (2017) diferenciam modelos baseados em dados (rede neural é um exemplo) de modelos baseados em técnicas experimentais e teóricas (exemplo: EIS) que são mais custosos. Por isso o teste mais comum é a medição da soma de todas as resistências internas como é feito por Saha, Goebel e Christophersen (2009).

\subsection{Cálculos de resistência e capacitância usando o ADC do microcontrolador}

Para estimar as resistências e a capacitância do modelo de Randle, a tensão da bateria foi medida de $20 \mathrm{em} 20$ milissegundos pelo ADC do microcontrolador depois de inserir uma resistência conhecida. Essa diferença de tempo foi escolhida para que o ADC possa medir e o microcontrolador registrar a tensão da bateria. Essa técnica é parecida à técnica descrita por Buchmann (2015) e utilizada por Saha, Goebel e Christophersen (2009).

O objetivo é estimar a resistência $R_{E}$, a resistência $R_{D}\left(R_{D}=R_{C T}+R_{W}\right)$ e a capacitância CDL. A resistência de carga $R c$ escolhida foi de $9,333 \Omega$, formada por seis resistores de $56 \Omega$ em paralelo. O valor de Rc foi escolhido para que a queda de tensão fosse grande o suficiente para ser medida pelo ADC. O circuito equivalente é o da figura 24 , gerado no software Pspice. Os valores de $R_{E}, C$ e RD são os valores medidos de uma bateria usada como exemplo. $O$ valor da tensão em aberto da bateria escolhido $(4,19 \mathrm{~V})$ é o mesmo da tensão de corte da carga das baterias. E o valor de "tClose $=20 \mathrm{~ms}$ " indica quando a chave é fechada. 
Figura 24 - Circuito modelo do teste para estimar os parâmetros da bateria.

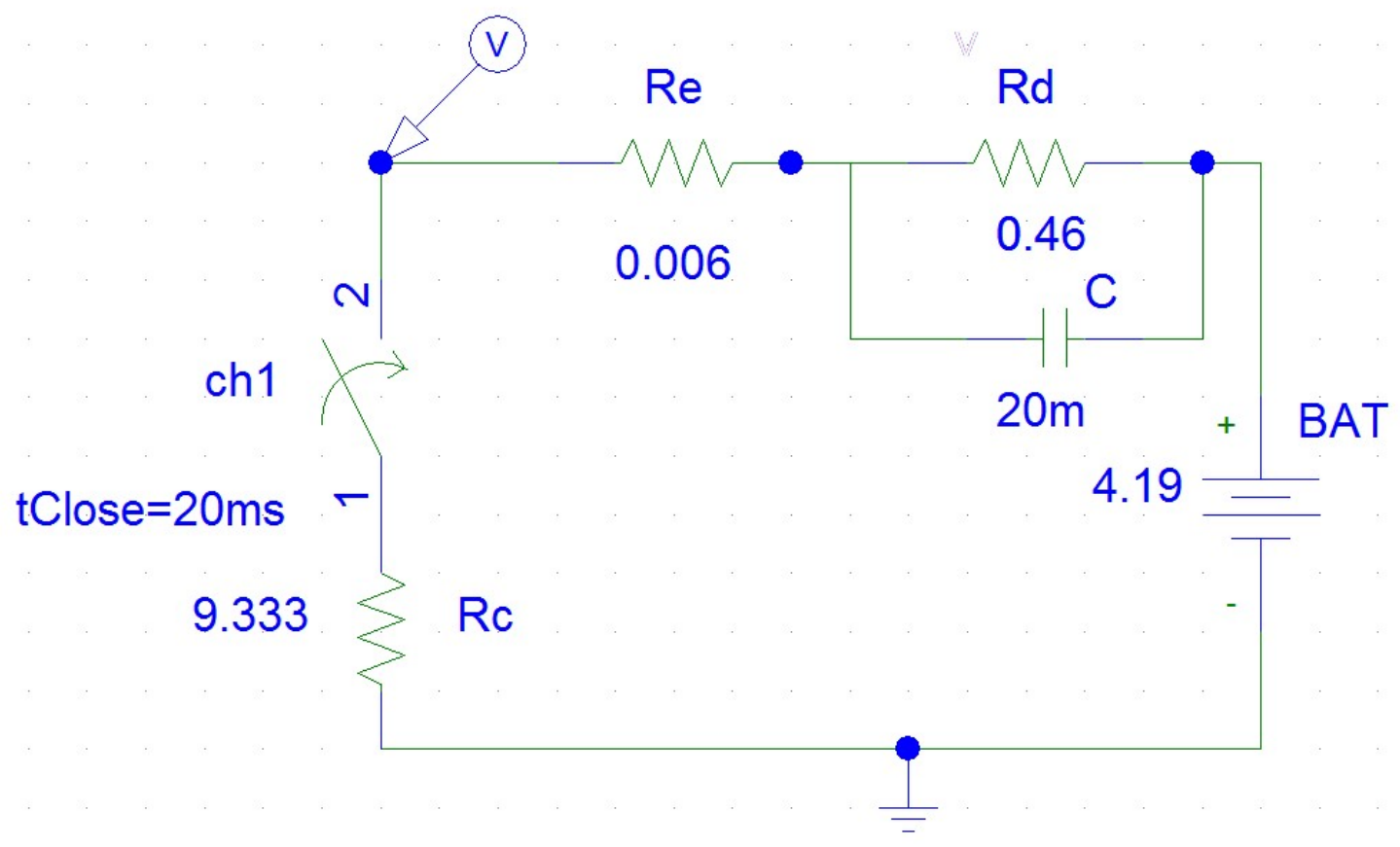

Fonte: Autor

No Pspice foi gerado o gráfico da figura 25 simulando a curva de tensão na saída da bateria (ponto "V" na figura 24).

Figura 25 - Tensão na saída da bateria durante o teste.

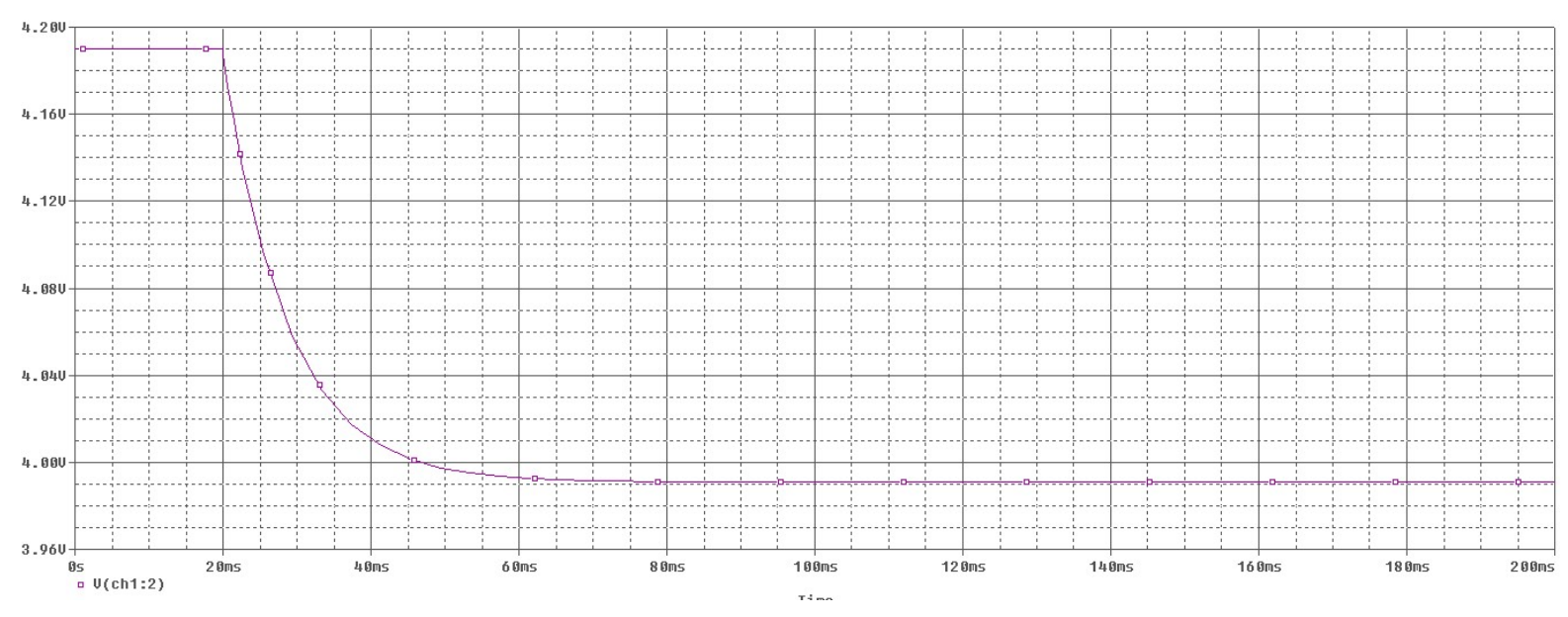

Fonte: Autor

O capacitor se comporta como um curto no instante $\mathrm{t}=0^{+}$, portanto há uma queda imediata na tensão de saída até uma tensão $V_{d}$. Essa tensão é representada pela fórmula da equação 3.1 . 


$$
V d=\frac{V_{B A T} \times R c}{R e+R c}
$$

Como é conhecido Rc é possível determinar Re com a equação 3.2.

$$
R e=\frac{R c \times\left(V_{B A T}-V d\right)}{V d}
$$

Após a carga completa do capacitor, a tensão de saída da bateria VBAT só depende das resistências internas, portanto a tensão de regime $V_{x}$ é calculada pela equação 3.3.

$$
V x(\infty)=\frac{V_{B A T} \times R c}{R d+R e+R c}
$$

Considerando $\mathrm{Rx}=\mathrm{Rd}+\mathrm{Re}$, é obtido o valor de $\mathrm{Rx}$ pela equação 3.4.

$$
R x=\frac{R c \times\left(V_{B A T}-V x\right)}{V x}
$$

Para obter-se a equação da capacitância, é preciso definir o circuito equivalente de Thévenin para simplificar a análise. A resistência equivalente de Thévenin Rth é definida pela resistência equivalente entre os pontos A e B do circuito da figura 26.

Figura 26 - Circuito equivalente para o cálculo da resistência de Thévenin.

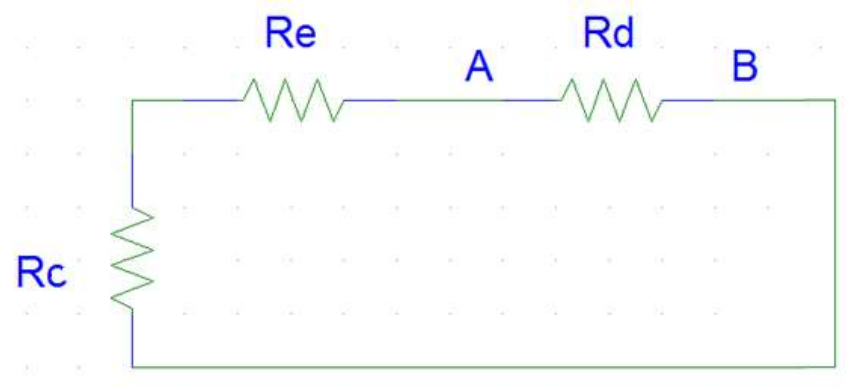

Fonte: Autor

Portanto Rth é definida pela equação 3.5.

$$
R t h=\frac{R d \times(R e+R c)}{R d+R e+R c}
$$

A tensão de Thévenin é igual à tensão em aberto $\mathrm{V}_{\mathrm{BAT}}$ da bateria nesse caso. $\mathrm{O}$ circuito resultante é mostrado na figura 27. 
Figura 27 - Circuito equivalente de Thévenin.

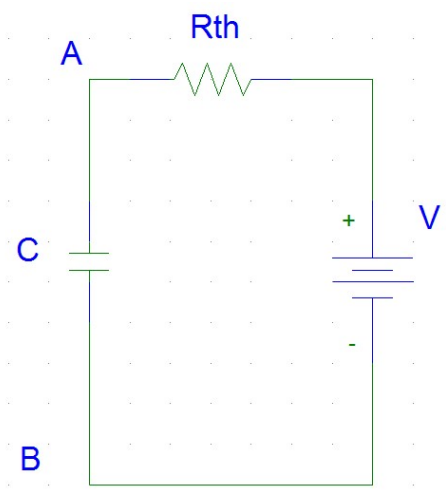

Fonte: Autor

A tensão $\mathrm{Vc}(\mathrm{t})$ no capacitor terá a fórmula 3.6.

$$
V c(t)=V c(\infty) \times\left(1-e^{\frac{-t}{R t h \times C}}\right)
$$

Sendo $\mathrm{Vc}(\infty)$ definido pela fórmula 3.7.

$$
V c(\infty)=V_{B A T} \times\left(1-\frac{R e+R}{R d+R e+R c}\right)
$$

Assim, é definida a tensão de saída da bateria $V_{x}$ em função de $V_{c}$ pela equação 3.8.

$$
V x(t)=\left(\frac{R c}{R e+R c}\right) \times\left(V_{B A T}-V c(t)\right)
$$

Portanto é possível estimar a capacitância com a curva da tensão de saída da bateria. Para validar o método, comparou-se os valores medidos da tensão de uma bateria com simulações Spice empregando os valores de capacitância e resistência estimados. Os valores medidos estão na tabela 1 e os parâmetros calculados na tabela 2. 
Tabela 1 - Registro das tensões da bateria nos primeiros 400ms de teste exemplo

\begin{tabular}{|c|c|}
\hline Tempo em milissegundos & Tensão medida em Volts \\
\hline 0 & 4.159 \\
\hline 20 & 4.156 \\
\hline 40 & 4.037 \\
\hline 60 & 4.035 \\
\hline 80 & 4.035 \\
\hline 100 & 4.035 \\
\hline 120 & 4.035 \\
\hline 140 & 4.035 \\
\hline 160 & 4.037 \\
\hline 180 & 4.034 \\
\hline 200 & 4.034 \\
\hline 220 & 4.035 \\
\hline 240 & 4.034 \\
\hline 260 & 4.037 \\
\hline 280 & 4.035 \\
\hline 300 & 4.037 \\
\hline 320 & 4.035 \\
\hline 340 & 4.03 \\
\hline 360 & 4.032 \\
\hline 380 & 4.032 \\
\hline & \\
\hline & Fontor \\
\hline
\end{tabular}

Fonte: Autor

Tabela 2 - Valores estimados do modelo de Randle de um teste exemplo.

\begin{tabular}{|c|c|}
\hline Parâmetros & Valor \\
\hline Re & $0.006737 \Omega$ \\
\hline Rd & $0.281358 \Omega$ \\
\hline Rx & $0.288095 \Omega$ \\
\hline Rc & $9.333333 \Omega$ \\
\hline Vbat & $4.159 \mathrm{~V}$ \\
\hline C & $0.018918 \mathrm{~F}$ \\
\hline
\end{tabular}

Fonte: Autor

Com esses valores, é obtido o gráfico medido pelo microcontrolador e o gráfico da simulação do Pspice para comparar na figura 28 : 
Figura 28 - Comparação entre a simulação e os valores medidos.

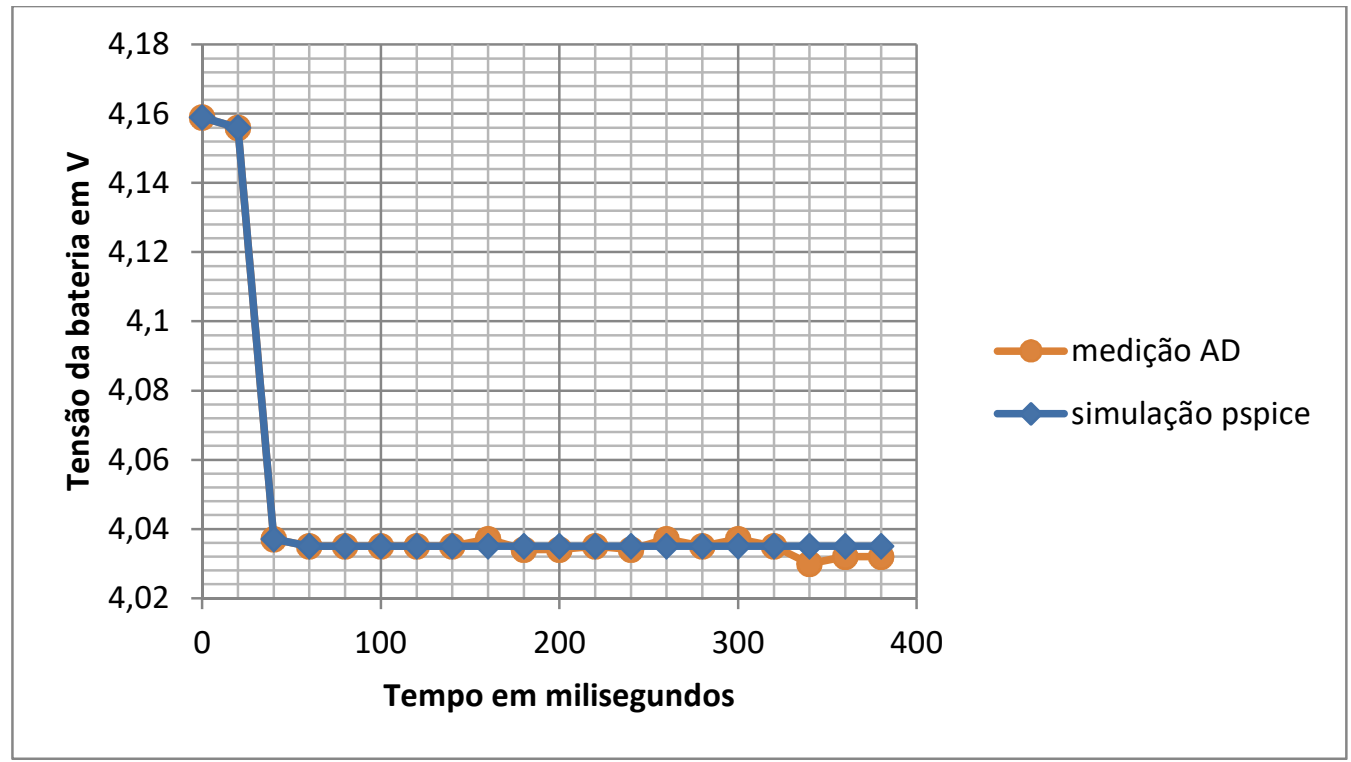

Fonte: Autor

\subsection{Descrição do Hardware}

Um microcontrolador foi utilizado para realizar as medições descritas anteriormente. O microcontrolador utilizado foi o LPC1778 da NXP, um microcontrolador de arquitetura ARM Cortex M3. As especificações deste microcontrolador estão no anexo D. Seria possível realizar as mesmas operações com um microcontrolador com uma capacidade computacional mais simples, já que para as únicas funcionalidades necessárias são uma medição de ADC e pinos para comunicar com uma memória externa. Uma EEPROM de 512kB de memória foi utilizada para armazenar os dados que eram transferidos para o PC via USB. Além disso, foi utilizado uma fonte externa AC/DC de 24V que alimentava o circuito de carga e descarga da bateria e a fonte de alimentação do microcontrolador. O circuito de carga está na figura 29: 
Figura 29 - Circuito de carga das baterias.

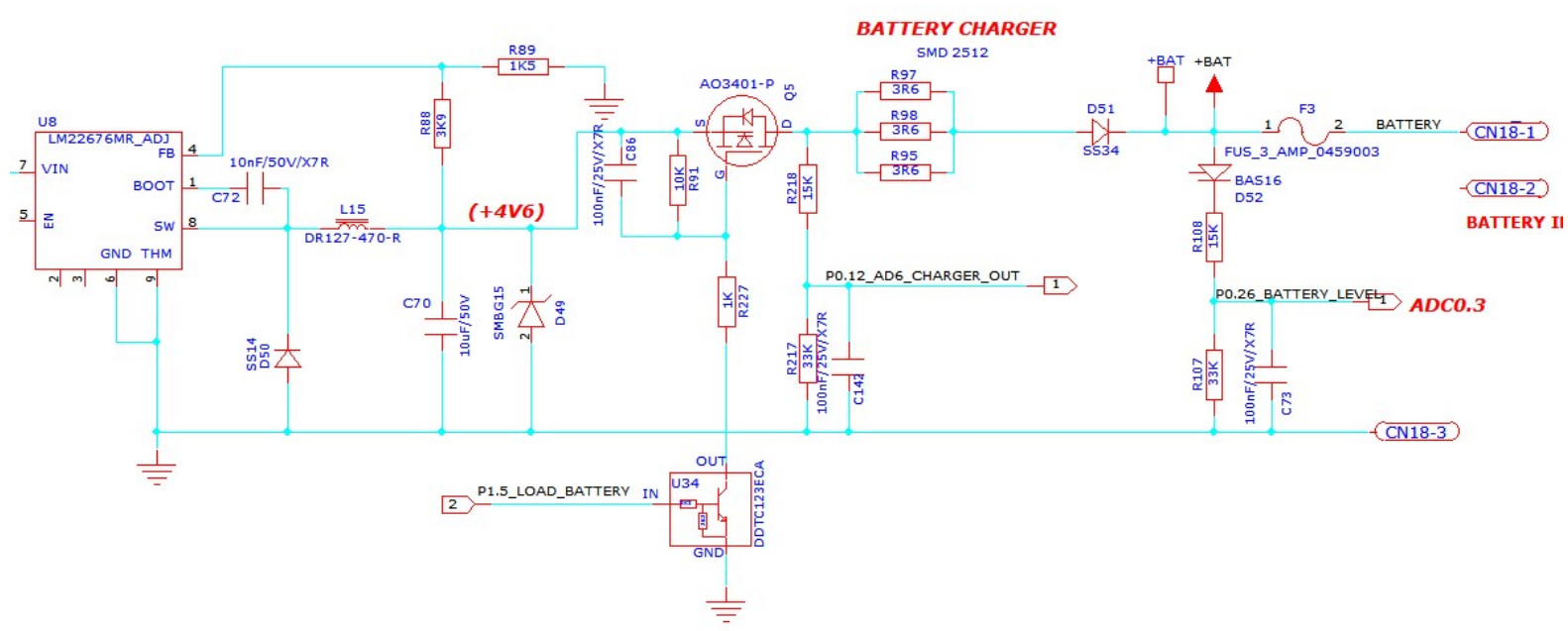

Fonte: Autor

O sinal "LOAD_BATTERY" é usado para chavear o carregador controlando assim a corrente de carga e mantendo a potência dissipada nos resistores de carga. $\mathrm{O}$ circuito de descarga tem funcionamento parecido como é mostrado figura 30 :

Figura 30 - Circuito de descarga das baterias.

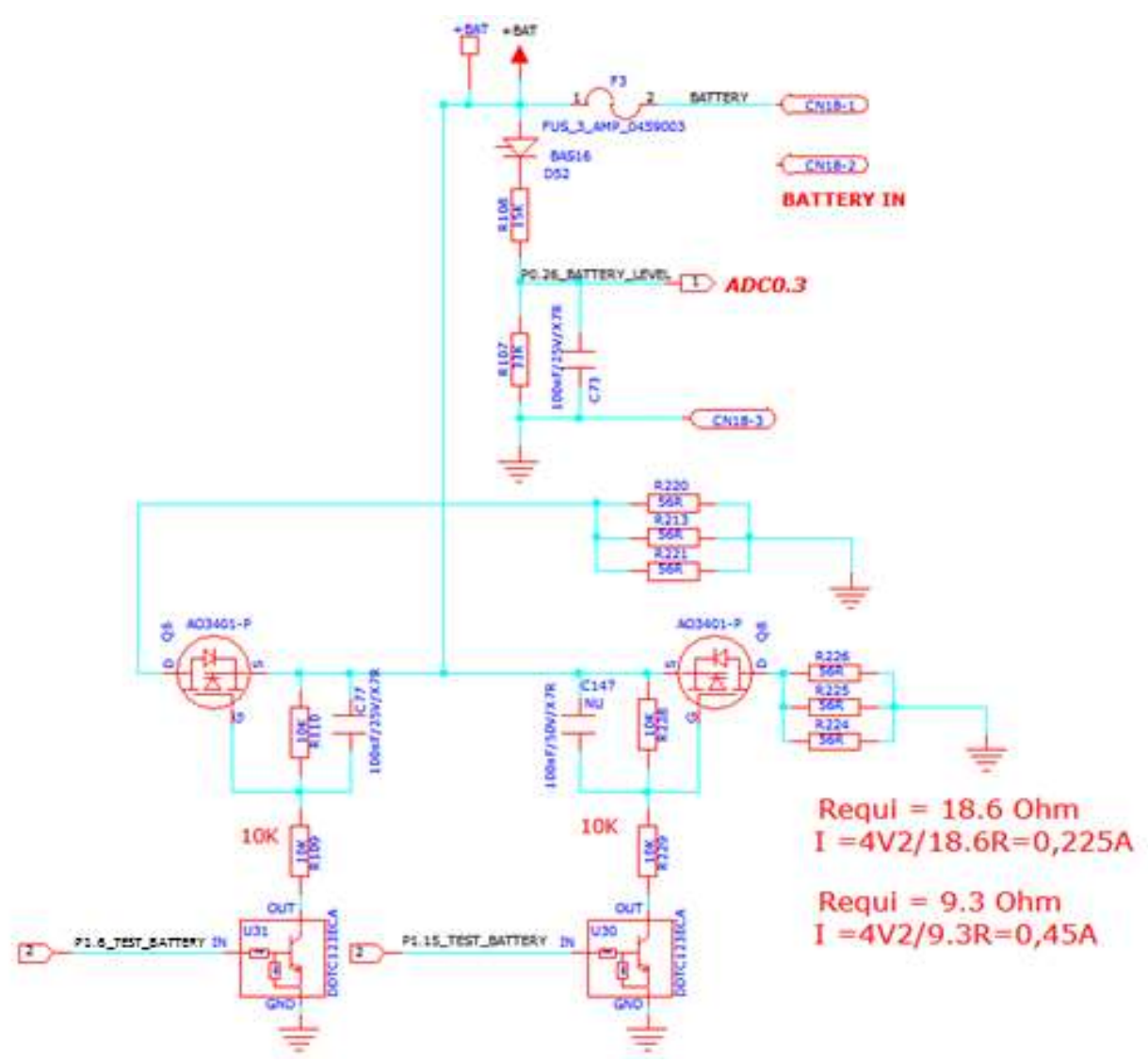

Fonte: Autor 
Os sinais "P1.6_TEST_BATTERY" e "P1.15_TEST_BATTERY" são usados para controlar a corrente média de descarga em $250 \mathrm{~mA}$ ou em $450 \mathrm{~mA}$.

21 baterias de três tipos diferentes foram testadas, todas de lítio polímero:

7 baterias da HCT (PN: HCT053759P) de 1150mAH.

$>7$ baterias da Expower (PN: 483757) de 1000mAH.

$>7$ baterias da Expower (PN: 483759) de 1000mAH.

Os datasheets dessas baterias se encontram em anexo (anexos A, B e C).

Na figura 31 é mostrada uma foto de todos os modelos de baterias que são todas do tipo pouch:

Figura 31 - Foto dos 3 tipos de baterias testadas.

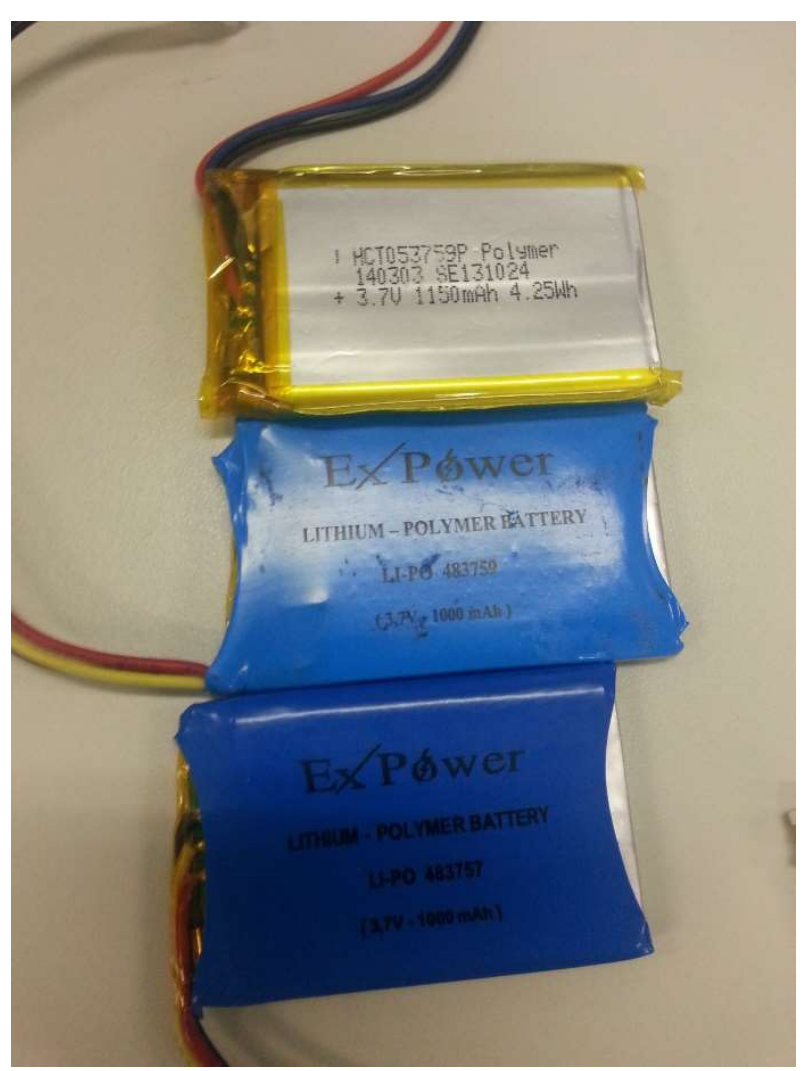

Fonte: Autor 


\subsection{Descrição do firmware}

O firmware de teste possui três conjuntos de funções. Extratos do FW estão no apêndice $A$.

\section{Função de log}

A leitura do log é executada no fim de cada ciclo de carga e descarga. O log é enviado via USB para o PC e esse log é tratado por um programa de planilhas. O log possui até 2048 registros de 8 bytes cada. Cada registro possui os seguintes parâmetros:

- Hora do log

- Minuto do log

- Segundo do log

- Registro não utilizado

- Valor do ADC da bateria ( $1^{\circ}$ byte)

- Valor do ADC da bateria ( $2^{\circ}$ byte)

- Valor do ADC do carregador da bateria ( $1^{\circ}$ byte)

- Valor do ADC do carregador da bateria $\left(2^{\circ}\right.$ byte $)$

Apesar de registrar o valor do ADC do carregador da bateria ("AD6_Charger_Out"), essa informação não é considerada na análise. Antes de cada ciclo de carga e descarga, o log é apagado.

Nos primeiros 400 milissegundos de descarga, o valor do log é computado, mas os bytes que indicam o horário são ignorados já que são irrelevantes para essas medidas.

\section{Função de carga}

O FW lê a tensão da bateria para determinar qual é a corrente de carga atual. O objetivo é limitar a potência média nos resistores de carga. A cada 30 segundos um log é registrado. A curva de carga não é importante para a análise, mas é importante que todas as baterias tenham sido carregadas da mesma forma, já que a forma de carregar influencia na capacidade da bateria. Quando a tensão da bateria atinge 4,19V o FW inicia automaticamente a função de descarga. Esse valor foi escolhido pois inicialmente a carga seria realizada com o carregador de baterias MCP73871 da Microchip e este é valor de carga máxima desse componente. Ao mudar a forma de carga das baterias, manteve-se o valor. 


\section{Função de descarga}

Antes da descarga, o carregador é desligado e a bateria é mantida em aberto por 2 segundos para que o carregador não influa na medida. $O$ valor da bateria é registrado antes de iniciar a descarga. Nos primeiros 400ms a bateria é descarregada com 450mA para medir a resistência e capacitância do modelo de Randle e durante esse processo o valor da tensão da bateria é armazenada na memória a cada $20 \mathrm{~ms}$. $\mathrm{Na}$ segunda etapa, a bateria é descarregada com uma corrente média de 250mA. Para garantir essa corrente média, o FW controla a descarga da bateria chaveando os sinais "P1.6_TEST_BATTERY" e "P1.15_TEST_BATTERY" com uma forma de onda quadrada de período de 50 milissegundos. O fator de ciclo (duty cycle) desse sinal é definido pela tensão da bateria para que a corrente de descarga seja próxima de $250 \mathrm{~mA}$ e todas as baterias sejam descarregadas da mesma forma. A tabela 3 mostra os valores de fator de ciclo e as respectivas correntes de descarga.

Tabela 3 - Definição do fator de ciclo da descarga.

\begin{tabular}{|c|c|c|c|}
\hline Tensão da bateria em Volts & $\begin{array}{c}\text { Tempo em } \\
\text { estado ativo } \\
\text { em } \\
\text { milissegundos }\end{array}$ & $\begin{array}{l}\text { Fator de ciclo em } \\
\text { porcentagem }\end{array}$ & $\begin{array}{c}\text { Corrente de descarga em } \\
\text { miliAmperes }\end{array}$ \\
\hline 3 & 39,00 & 0,78 & 252 \\
\hline 3,06 & 38,00 & 0,76 & 250 \\
\hline 3,12 & 37,00 & 0,74 & 248 \\
\hline 3,18 & 37,00 & 0,74 & 253 \\
\hline 3,24 & 36,00 & 0,72 & 251 \\
\hline 3,3 & 35,00 & 0,7 & 248 \\
\hline 3,36 & 35,00 & 0,7 & 253 \\
\hline 3,42 & 34,00 & 0,68 & 250 \\
\hline 3,48 & 33,00 & 0,66 & 247 \\
\hline 3,54 & 33,00 & 0,66 & 251 \\
\hline 3,6 & 32,00 & 0,64 & 248 \\
\hline 3,66 & 32,00 & 0,64 & 252 \\
\hline 3,72 & 31,00 & 0,62 & 248 \\
\hline 3,78 & 31,00 & 0,62 & 252 \\
\hline 3,84 & 30,00 & 0,6 & 248 \\
\hline 3,9 & 30,00 & 0,6 & 252 \\
\hline 3,96 & 29,00 & 0,58 & 247 \\
\hline 4,02 & 29,00 & 0,58 & 251 \\
\hline 4,08 & 28,00 & 0,56 & 246 \\
\hline 4,14 & 28,00 & 0,56 & 249 \\
\hline 4,2 & 28,00 & 0,56 & 253 \\
\hline
\end{tabular}

Fonte: Autor 
A cada 30 segundos a tensão da bateria é registrada no log e quando a tensão da bateria atinge $3 \mathrm{~V}$, o ciclo é interrompido.

\section{5. $\quad$ Tratamento dos dados experimentais}

O cálculo das resistências do modelo de Randle é feito em uma tabela de Excel. Um documento de Excel foi criado para cada bateria, assim é possível compilar todas as curvas de carga e descarga e compará-las. Além disso, são compiladas nesses documentos as curvas do início da descarga de cada bateria. Com a curva de carga e descarga é possível determinar a capacidade da bateria pelo tempo de descarga, e assim estimar o $\mathrm{SOH}$ da bateria. 


\section{Análise dos dados obtidos}

\subsection{Análise inicial dos dados}

Foram feitas 1050 medições divididas em 50 medições por bateria.

Para cada bateria foi gerado um gráfico com as curvas de carga e descarga e as curvas dos primeiros $400 \mathrm{~ms}$ de descarga. A seguir, nas figuras 32 e 33 são apresentados esses gráficos para a bateria 2 .

Figura 32 - Curvas de carga e descarga da bateria 2.

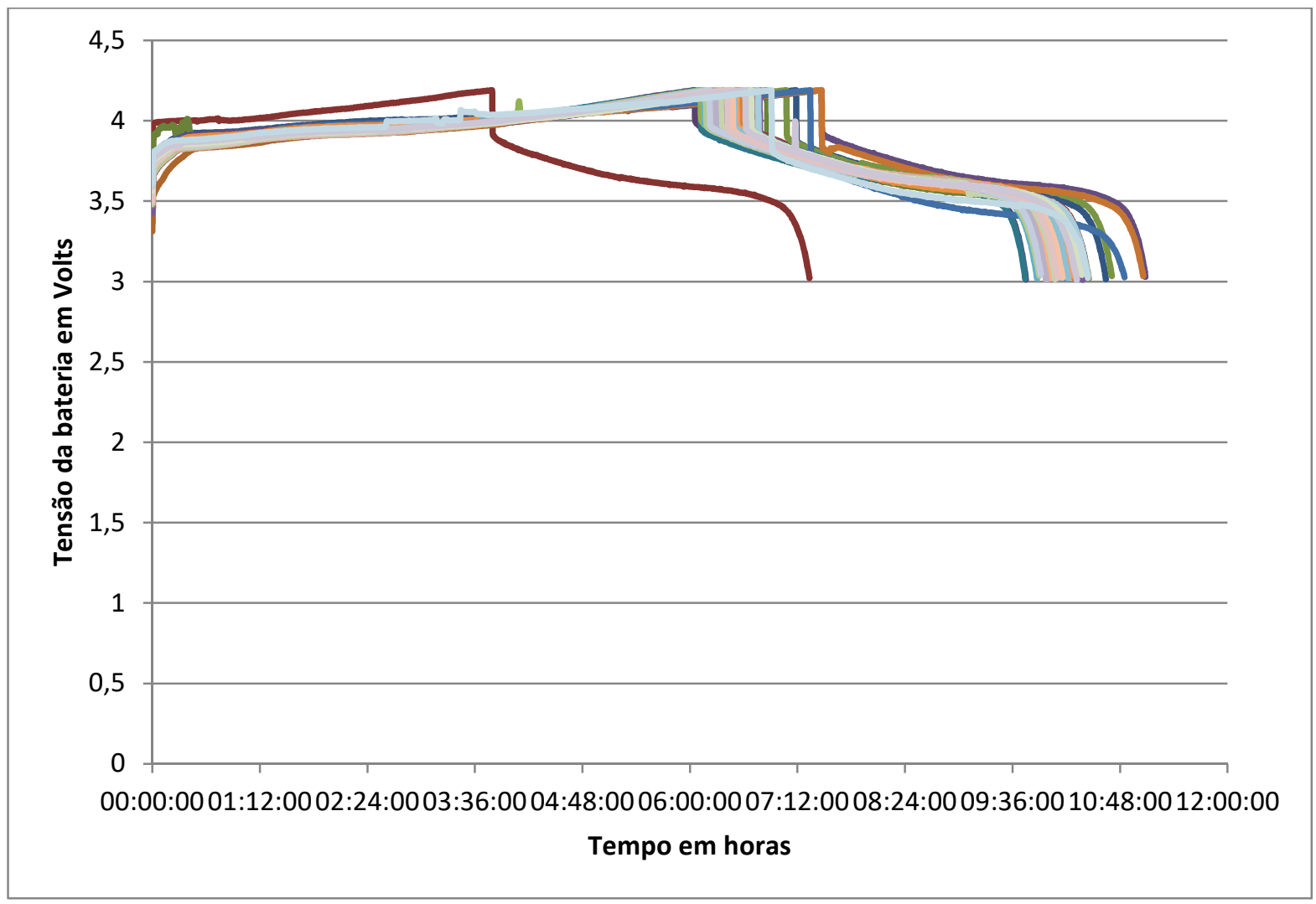

Fonte: Autor 
Figura 33 - Curvas do início da descarga da bateria 2.

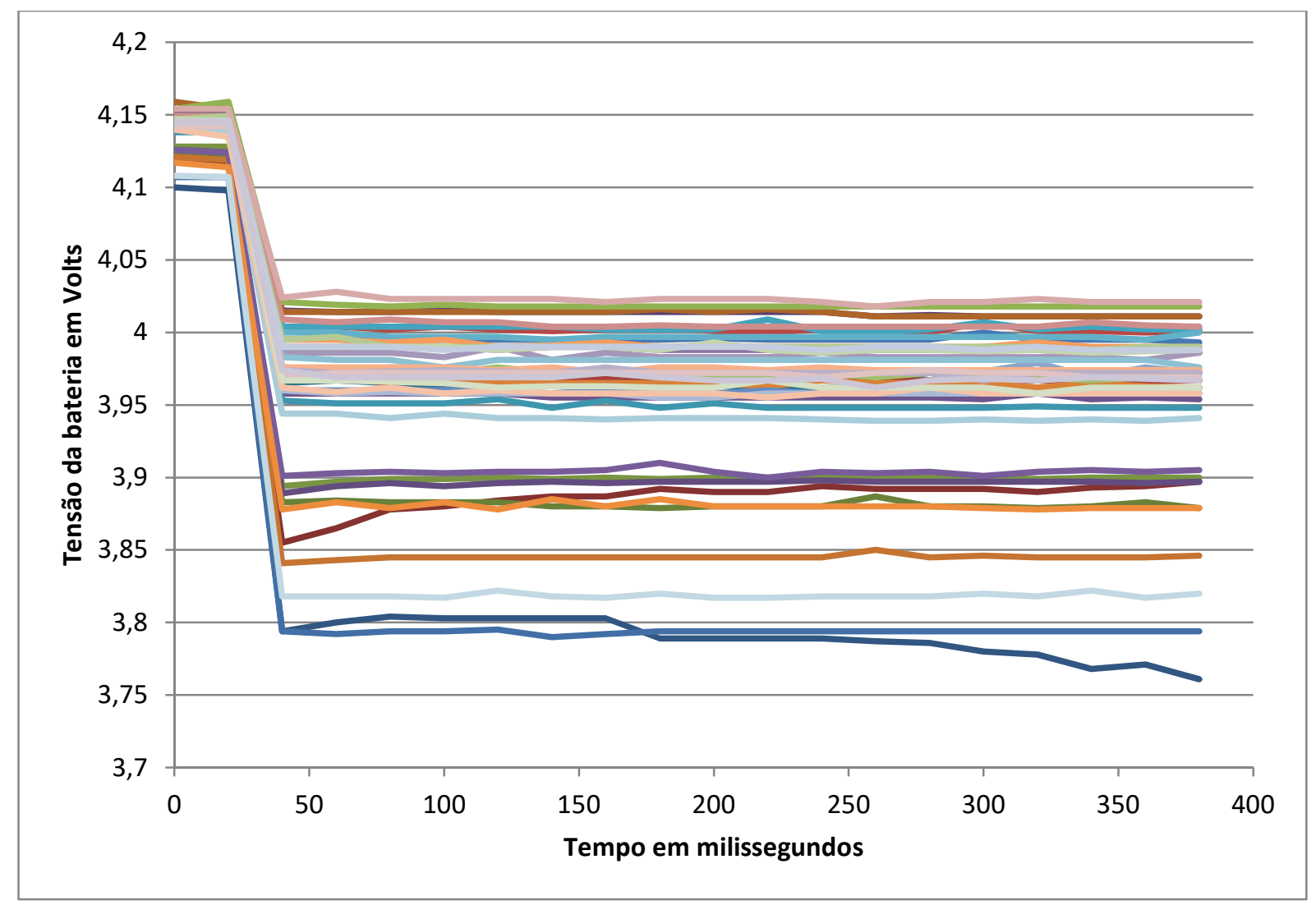

Fonte: Autor

A primeira curva de carga e descarga tem um tempo de carga menor porque a bateria já estava carregada. O tempo de descarga dessa bateria permaneceu entre 3hs30 e 3hs50 com pequenas variações, ou seja, o envelhecimento desta bateria ainda está controlado, ou seja, está variando pouco entre cada medida e não chegou no fim de vida. Outras baterias analisadas tiveram mudanças maiores entre os tempos de descarga. Porém a mudança sempre foi percebida no início da descarga. Quanto maior a queda de tensão no início e por consequência uma maior resistência, menor era o tempo de descarga, portanto menor era a capacidade da bateria.

Os gráficos da capacitância (figura 34) e da resistência (figura 35) em função do SOH mostram essa evolução. Para realizar a análise da evolução da resistência, a resistência $\mathrm{Rx}$, ou seja, a soma das resistências internas foi escolhida para obter uma análise mais precisa, já que nos cálculos, a resistência Re é menor e muito próxima de zero. 
Figura 34 - Relação entre a capacitância e o SOH.

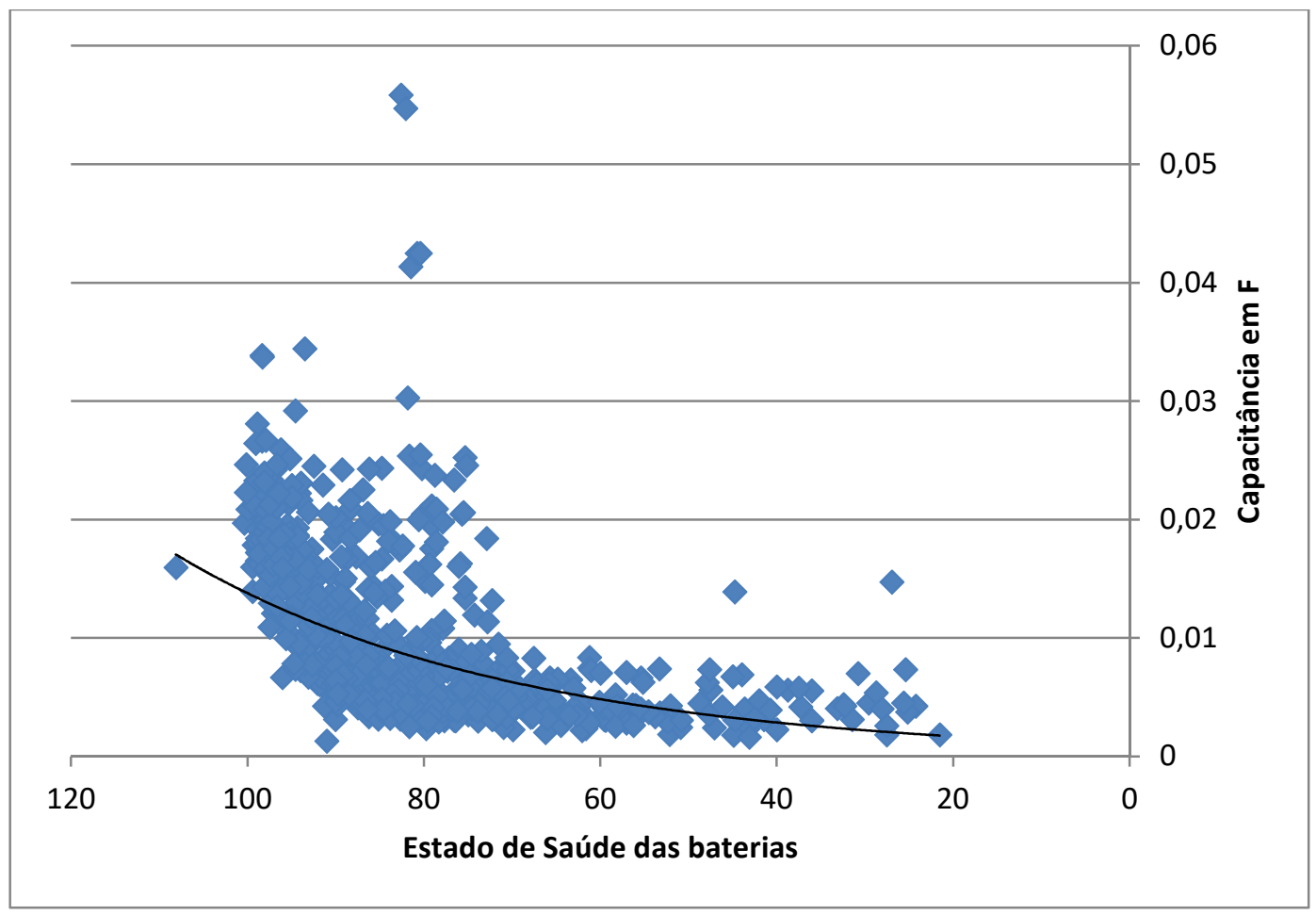

Fonte: Autor

Figura 35 - Relação entre a resistência Rx e o SOH.

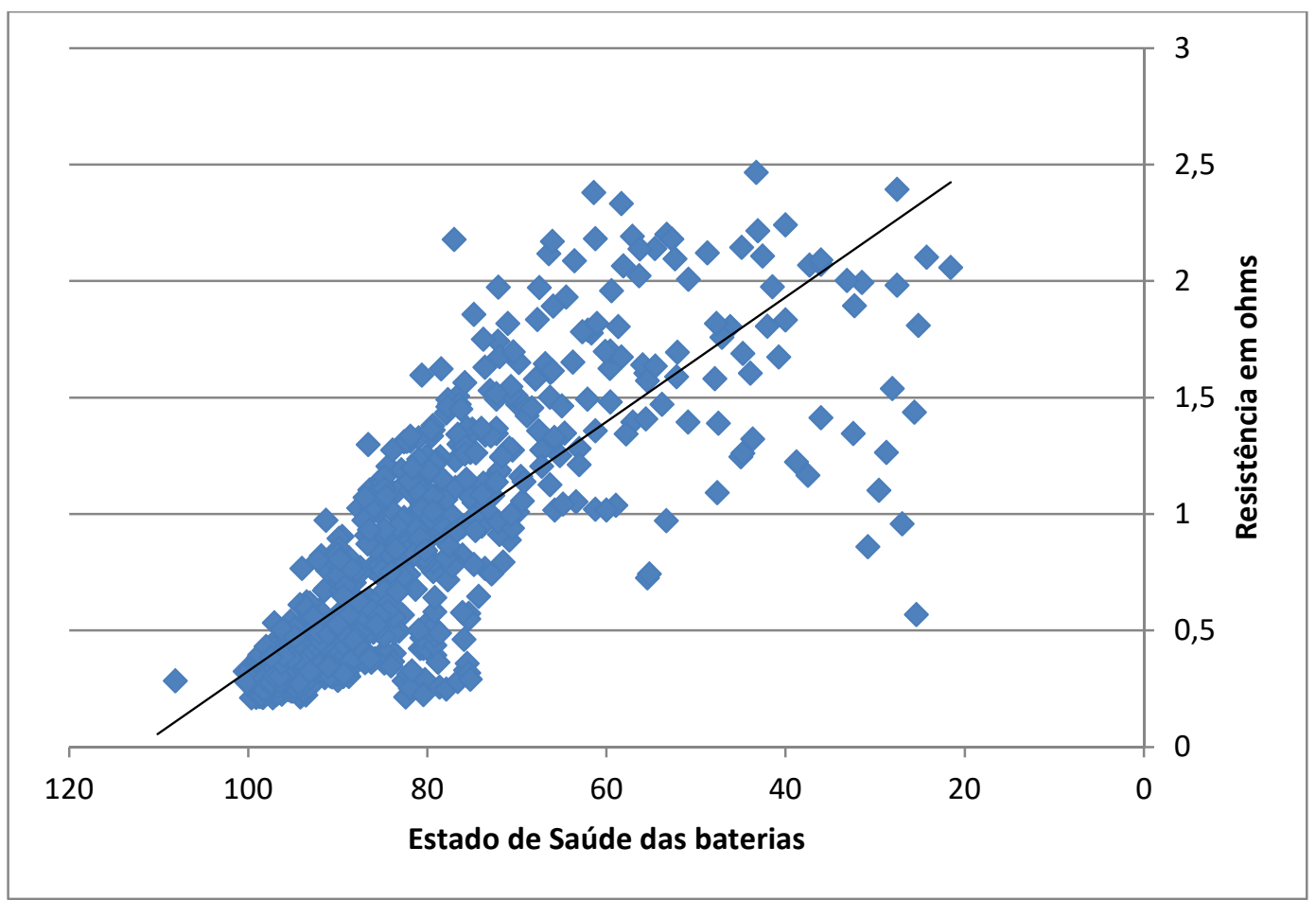

Fonte: Autor 
Os dados completos estão nos apêndices. Para comparar a resistência e capacitância entre os vários modelos de bateria, criou-se os gráficos das figuras 36 e 37, onde cada cor representa um modelo de bateria diferente.

Figura 36 - Relação entre a resistência Rx e o SOH dividido por modelo de bateria.

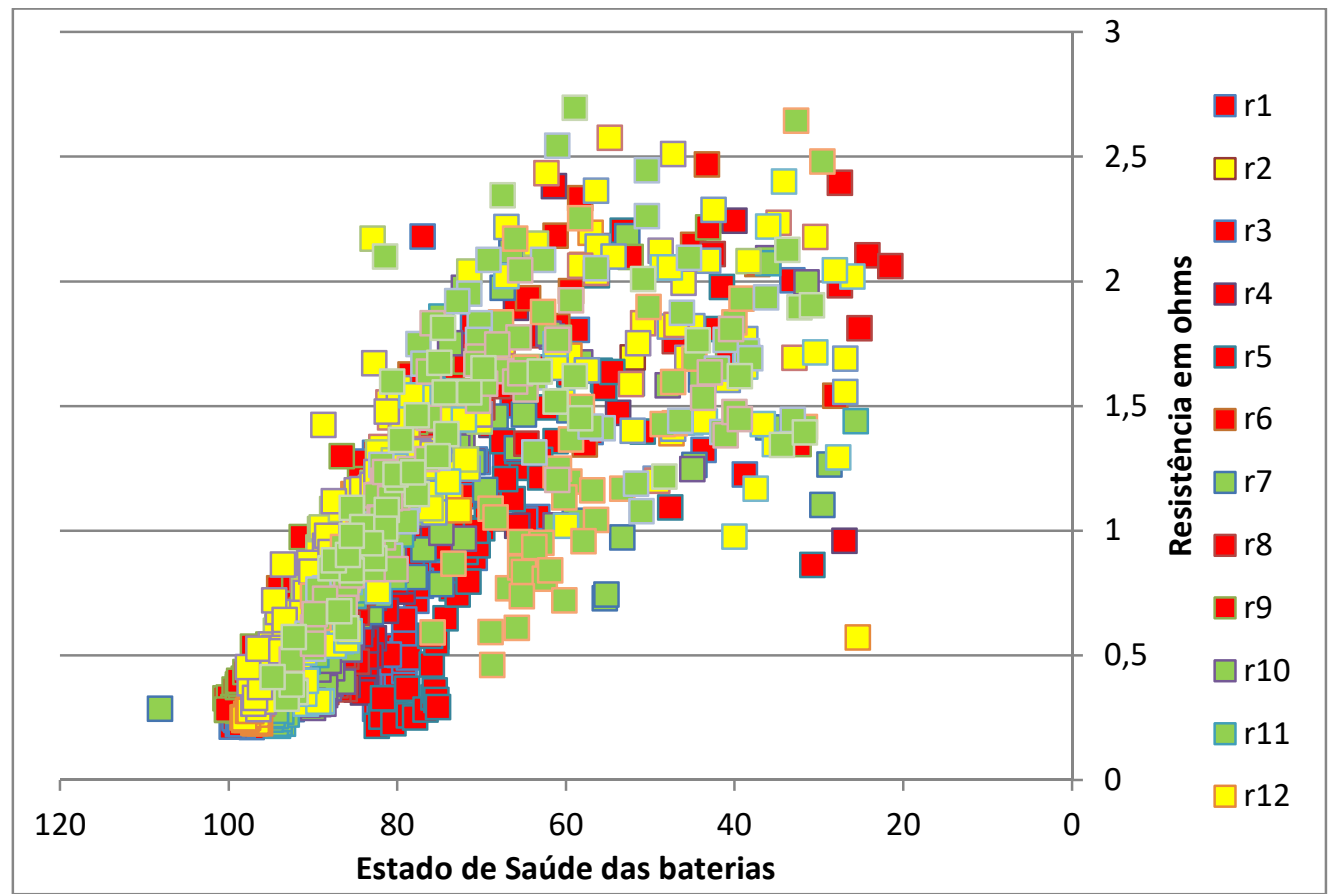

Fonte: Autor

Figura 37 - Relação entre a capacitância e o SOH dividido por modelo de bateria.

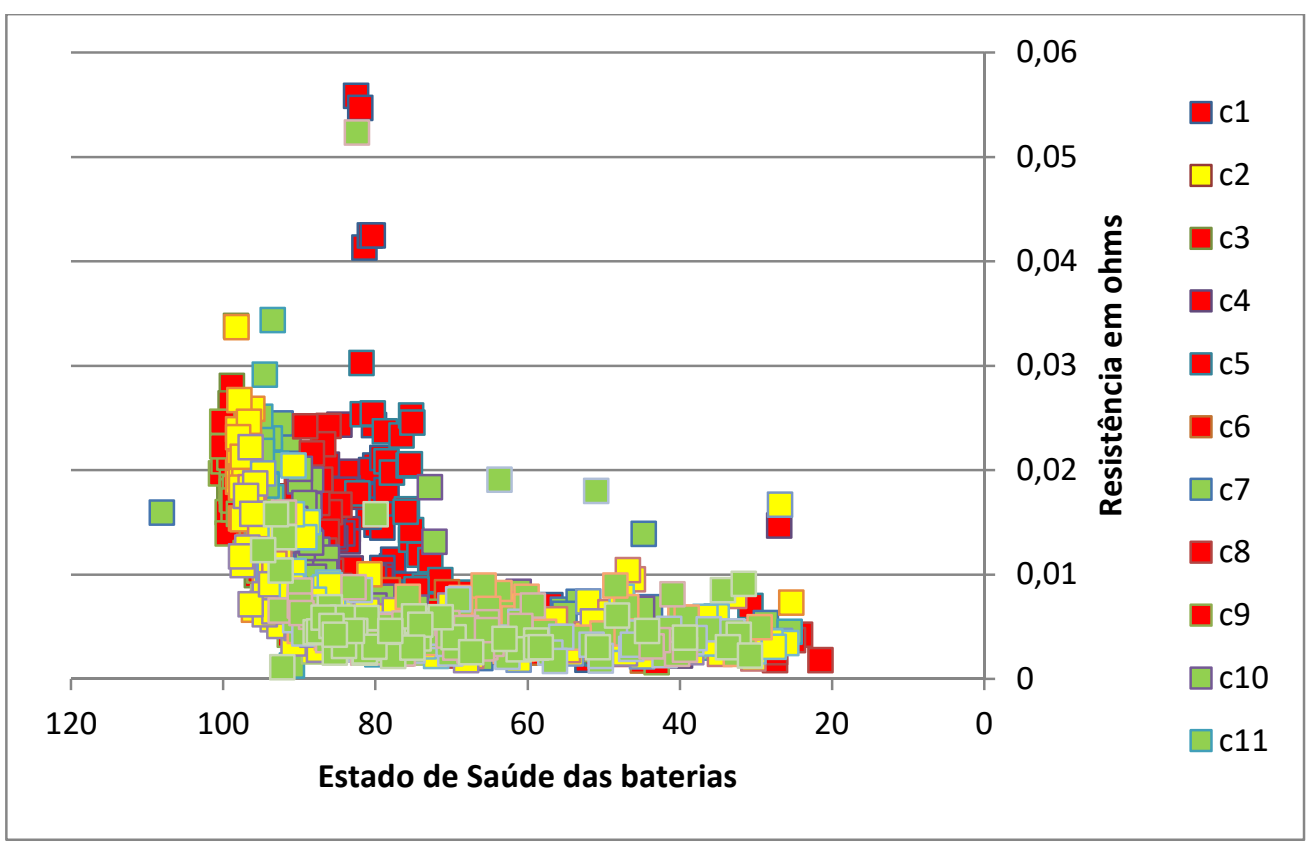

Fonte: Autor 
É possível observar que a resistência tem uma relação linear com o $\mathrm{SOH}$ e que a relação muda entre cada modelo de bateria, confirmando com o que é observado por Xing et al (2011). Por isso, é tão impreciso medir o SOH de uma bateria somente pela resistência. É possível analisar a evolução de uma bateria específica e estimar o seu $\mathrm{SOH}$ com o aumento da resistência interna, porém esse método não pode ser generalizado para um conjunto de baterias e, além disso, depende de uma confirmação do SOH real em cada medida. Esse método é inviável em muitas aplicações, principalmente em baterias estacionárias com ênfase em aplicações de segurança, onde não é possível descarregar a bateria para realizar os testes ou quando os testes devem ser realizados em tempo real.

A relação da capacitância e do SOH não é linear, porém é possível tirar as mesmas conclusões, pois há no gráfico áreas definidas pelos modelos das baterias. Portanto uma análise completa dos dados deveria levar em conta qual é o modelo da bateria, algo que não é factível em certas aplicações comerciais onde baterias de diferentes fabricantes são utilizadas.

\subsection{Problemas e imperfeições na medição}

Como foi mencionado anteriormente, o SOH é medido como a relação entre a capacidade atual da bateria e a capacidade nominal. O problema que isso causa para os testes realizados é que o $\mathrm{SOH}$ fica dependente da temperatura, da corrente de carga, da corrente de descarga e do modo que é realizada a carga (GOLD PEAK INDUSTRIES LTD, 2003; XING et al, 2011). Certas condições foram definidas para a realização dos testes, mesmo assim o $\mathrm{SOH}$ das baterias não evoluiu de uma maneira controlada nos testes realizados. O principal fator para isso deve ser a variação de temperatura já que os testes foram realizados em condições ambientes. Porém os valores dos componentes do modelo de Randle variaram de acordo ao tempo de descarga da bateria, mostrando-se, em um primeiro momento, uma forma eficiente de prever a capacidade atual da bateria. A capacidade de baterias com $\mathrm{SOH}$ baixo é instável, ou seja, a capacidade pode mudar de ciclo para ciclo. Algumas baterias foram escolhidas por estarem nessa condição, assim pode-se medir a resistência e capacitância para diferentes valores de SOH (de 20\% a 110\%). Foi medido valores de $\mathrm{SOH}$ acima de $100 \%$, e isso pode ser explicado por algumas baterias testadas serem novas e apresentarem capacidade um pouco acima da nominal. Isso acontece normalmente na primeira descarga. 
É possível visualizar essas imperfeições na diferença entre a variação do SOH em função dos ciclos de carga e descarga de duas baterias nas figuras 38 e 39 . Enquanto a bateria 3 apresenta uma diminuição do $\mathrm{SOH}$ regular até a $19^{\mathrm{a}}$ medida, o gráfico da bateria 1 é mais instável, mudando o $\mathrm{SOH}$ de ciclo a ciclo. Após a 19a medida, o gráfico da bateria 3 demonstra uma instabilidade, porém continua a sua tendência de queda. Essa diferença acontece mesmo com baterias do mesmo modelo (que é o caso das baterias 1 e 3). Porém é importante notar que a resistência e a capacitância medidas acompanham essas mudanças. Os valores das resistências e das capacitâncias estão em porcentagem em relação à primeira medida para melhor visualização:

Figura 38 - Evolução do SOH, da resistência Rx e da capacitância ciclo a ciclo da bateria 1.

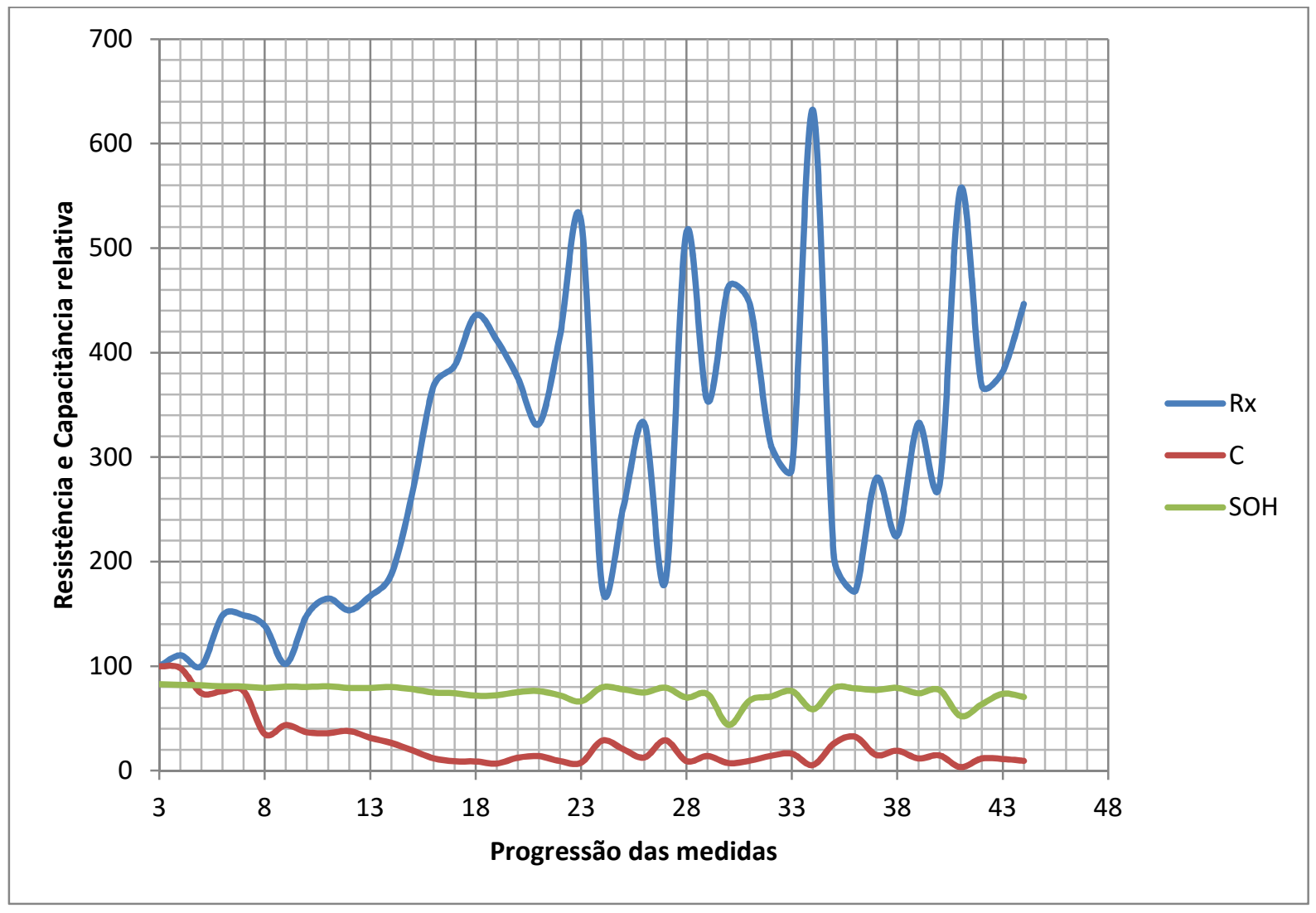

Fonte: Autor 
Figura 39 - Evolução do SOH, da resistência Rx e da capacitância ciclo a ciclo da bateria 3.

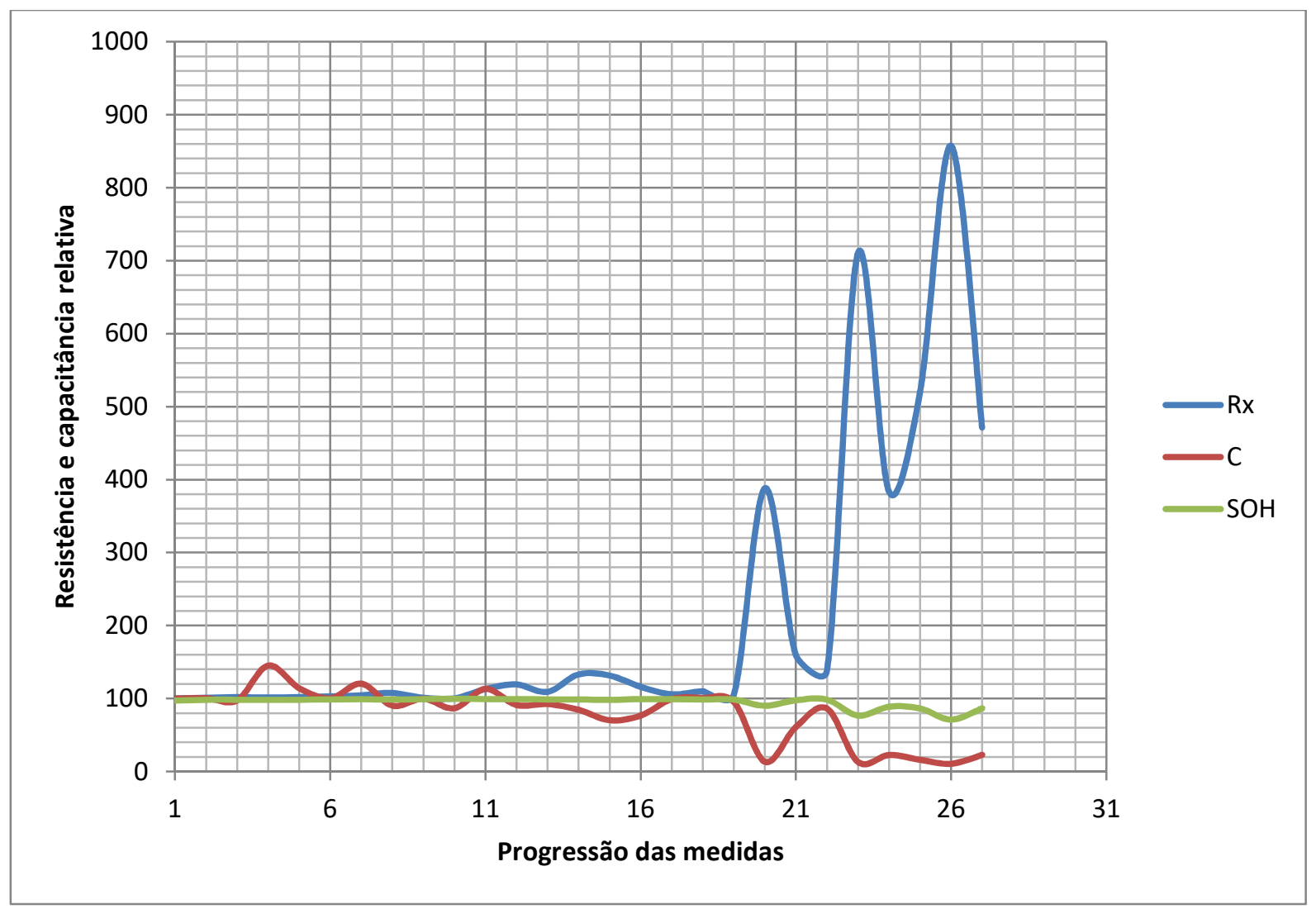

Fonte: Autor

Poucas medições atingiram $100 \%$ de SOH. O motivo principal foi que a tensão máxima de carga foi 4,19V e após a bateria atingir essa tensão, a carga foi interrompida. Normalmente, há uma segunda etapa da carga com tensão constante o que aumentaria a capacidade da bateria, porém aumentando ao mesmo tempo a taxa de envelhecimento. Ao carregar a bateria dessa maneira foi possível realizar testes em diversas faixas de $\mathrm{SOH}$.

As medições realizadas possuem outras limitações, como por exemplo, o tempo de medição do ADC. A medição não é imediata e tem um tempo de duração o que implica diretamente na estimativa dos componentes do modelo de Randle. O ciclo de medição (aquisição e gravação dos dados) não pode durar mais tempo que 20ms.

Além disso, o valor de Re é pequeno, por isso muitas vezes o valor estimado é $0 \Omega \mathrm{e}$ em alguns casos isso acarreta em erros nos cálculos. Por exemplo, a tensão medida entre o circuito em aberto e o tempo $t=0^{+}$sendo próxima de $0 \mathrm{~V}$, as vezes pode 
acontecer da segunda medição da tensão ser arredondada para cima, criando valores errados e negativos de $\operatorname{Re}$. Quando isso acontece, definiu-se $\operatorname{Re}=0$.

Outro problema acontece ao medir a tensão $\mathrm{Vx}(\infty)$, a tensão que o circuito se estabiliza depois de colocar a carga. Para fazer essa medição, é considerada a média das últimas 15 medidas, porém se essas medidas não variarem muito, esse método pode acarretar em erros na medição da capacitância do modelo.

Todos esses erros em certas medições são esperados já que foi escolhido um método de medição mais abrangente que prioriza a flexibilidade entre aplicações. Esses problemas ocorreriam em qualquer aplicação que tentar estimar esses parâmetros, portanto é necessário projetar soluções para reconhecer esses erros e corrigi-los. Em aplicações que possuem baterias estacionárias, esses problemas são facilmente contornados descartando as medidas e refazendo os testes já que os testes nesse tipo de bateria poderiam ser realizados em grandes intervalos de tempo. 


\section{Base teórica dos métodos}

\subsection{Redes neurais}

Mukherjee (2003) compila as diferentes publicações que usam redes neurais e lógica fuzzy em BMS, para medir principalmente o $\mathrm{SOC}$ e o $\mathrm{SOH}$ de baterias. Mukherjee (2003) e O'Gorman et al (2008) justificam que o uso de redes neurais para esse propósito é vantajoso porque a análise deixa de ser estática e depende menos dos dados adquiridos em laboratórios. Como os parâmetros variam de modelo para modelo e de bateria para bateria, o uso de redes neurais na análise seria uma maneira de contornar isso por ser um método adaptativo e como lembram Lu et al (2013) a rede neural não leva em consideração as particularidades de cada bateria, sendo assim um método mais generalista. Outra vantagem das redes neurais é ser um método que pode ser atualizado enquanto a aplicação está funcionando, se for possível comunicar com o microcontrolador. O'Gorman et al (2008) explicam outras vantagens das redes neurais como a baixa complexidade computacional desse método. Além disso, é dito que ao usar redes neurais não é necessário completo conhecimento de todos os processos químicos da bateria, nem é necessário descrever todos esses processos. Portanto usando redes neurais é possível economizar tempo na análise e na medição dos parâmetros (O'GORMAN et al, 2008). Uma desvantagem das redes neurais apontada por Lu et al (2013) é a dependência de uma grande base de dados por isso deve-se tomar cuidado para que a rede neural não seja dependente dos dados de treinamento evitando assim um sobre ajustamento (overfitting).

Chan, Lo e Shen (2000) usam uma rede neural para estimar a capacidade de uma bateria de chumbo ácido usando a corrente de descarga como entrada, a rede neural foi comparada com métodos usando a lei de Peukert. O'Gorman et al (2008) usam uma rede neural para simular o comportamento de uma bateria durante a descarga. O objetivo dessa rede neural é ser um modelo eletroquímico que conseguiria prever o comportamento da bateria com diferentes correntes de descarga.

A base de uma rede neural é um perceptron composto por um vetor $\{X\}$ de entrada $\left(\mathrm{X}_{1}, \mathrm{X}_{2}, \ldots, \mathrm{X}_{\mathrm{N}}\right)$. Cada entrada $\mathrm{X}_{\mathrm{N}}$ é multiplicada por um peso $\mathrm{W}_{\mathrm{N}}$ do vetor de pesos $\{\mathrm{W}\}$ e somada como exemplifica a figura 40 : 
Figura 40 - Modelo de um perceptron de uma rede neural.

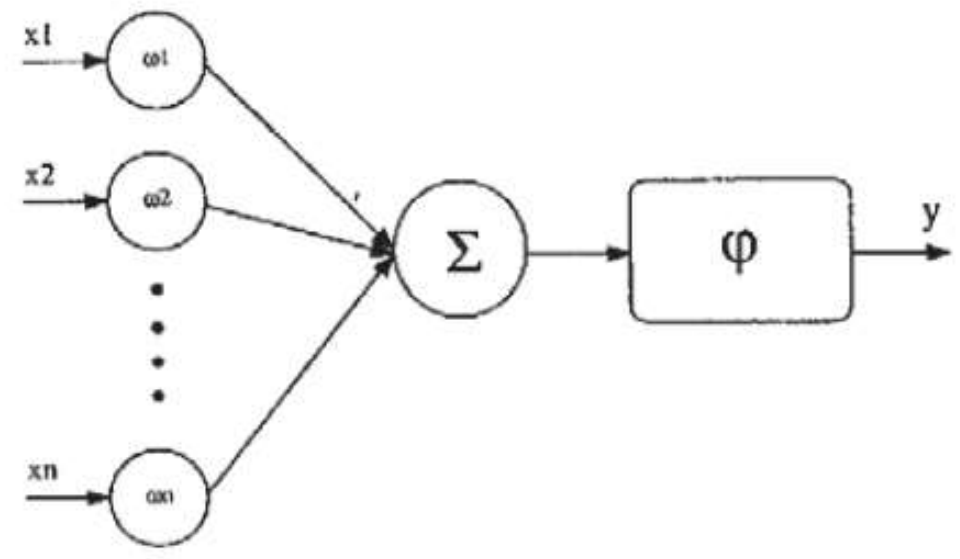

Fonte: Mukherjee (2003)

Depois da soma, há a função de ativação da rede neural. A função de ativação mais simples tem como saída "um" quando a soma passa por um determinado valor ou "zero". Segundo Mukherjee (2003) a função mais usada em redes neurais é a função sigmoide.

Para definir o vetor $\{W\}$ de pesos é preciso treinar o perceptron pela lei de treinamento do perceptron (Paplinski, 2005). O erro $\varepsilon(n)$ é defenido pela diferença entre a saída $d(n)$ esperada e o $y(n)$ calculado. Os pesos são corrigidos seguindo a seguinte equação:

$$
w(n+1)=w(n)+\eta \varepsilon(n) x(n)
$$

Onde $\eta$ é o ganho que controla taxa de aprendizado e precisa ser ajustado para garantir que o algoritmo convirja para o valor desejado. A cada interação o peso w é atualizado.

Esse método é adaptado para definir o vetor ótimo $\{W\}$ que minimiza o erro. Portanto o erro é calculado para cada peso do vetor e o objetivo é minimizar o erro médio quadrático dado pela equação 5.2 .

$$
J(w)=\frac{1}{2 N} \sum_{n=1}^{N} \varepsilon^{2}(n)
$$

O gradiente $\nabla \mathrm{J}(\mathrm{w})$ de $\mathrm{J}$ em relação à w é calculado para achar um mínimo local ou global de $\mathrm{J}(\mathrm{w})$. Esse mínimo é encontrado quando $\nabla \mathrm{J}(\mathrm{w})=0$. Para tanto, caminha-se 
na direção que $\nabla \mathrm{J}(\mathrm{w})$ é negativo, o método do gradiente decrescente. Esse processo é mostrado na equação 5.3 e na figura 41.

$$
\Delta w=-\eta \nabla J(w)
$$

Figura 41 - llustração do método de gradiente decrescente.

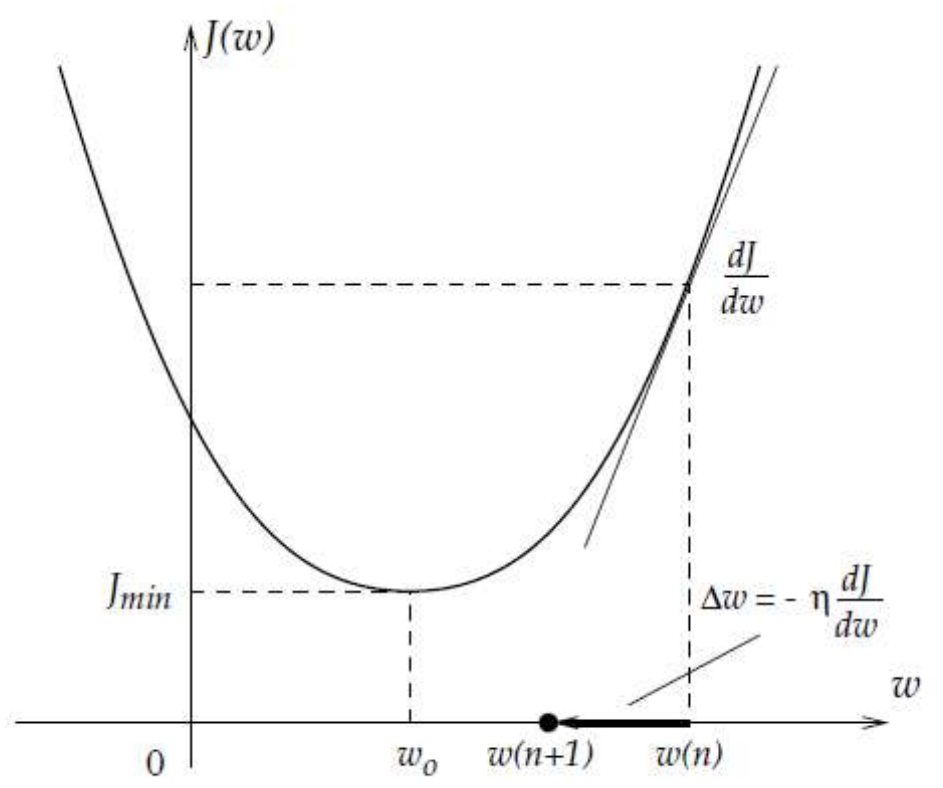

Fonte: Paplinski (2005)

Se esse ponto não é atingido, é necessário um critério de parada. Segundo Paplinski (2005), há dois critérios de parada mais populares: quando o erro é pequeno o suficiente ou quando a variação entre interações é suficientemente pequena.

Para simular funções não lineares é usada uma camada interna antes da camada de saída. Segundo O'Gorman et al (2008) e Paplinski (2005), duas camadas (uma interna e uma de saída) são suficientes para aproximar qualquer função não linear desde que se tenham dados suficientes para o treinamento. A figura 42 mostra uma rede neural com uma camada interna: 
Figura 42 - Rede neural com uma camada interna.

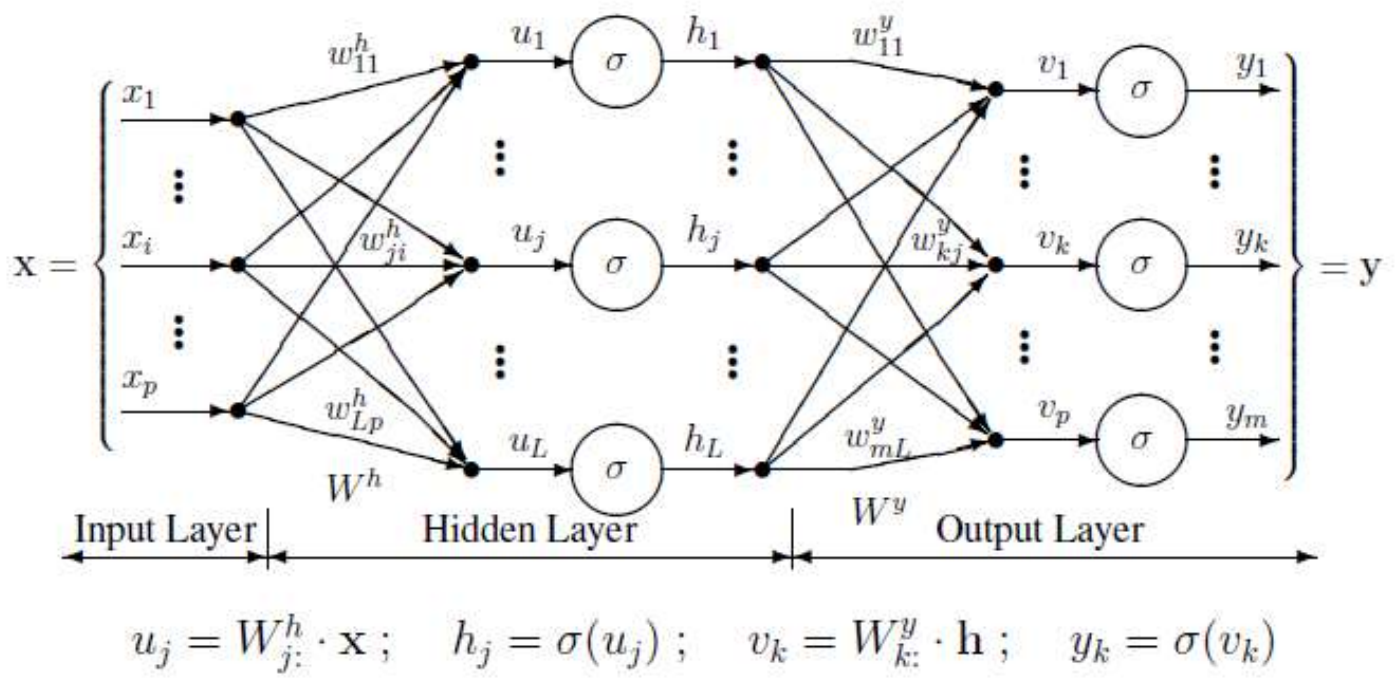

Fonte: Paplinski (2005)

Para treinar uma rede neural de multicamadas é necessário propagar o erro nas outras camadas (backpropagation) atualizando os pesos da camada interna. Portanto, os pesos de uma camada dependem dos valores da outra camada. Assim, é possível atualizar os pesos depois de cada dado de treinamento ou depois de todos os dados de treinamento forem usados (PAPLINSKI, 2005).

O número de nós em cada camada muda em cada caso. A rede neural usada por Chan, Lo e Shen (2000), por exemplo, possui uma camada interna com quatro nós. Segundo os autores esse modelo foi escolhido, pois apresentava uma melhor taxa de convergência e precisão. Sbarufatti et al (2017) explicam que um maior número de neurônios internos aumenta a capacidade e complexidade da rede neural, porém há o risco de sobre ajustamento (overfitting), o que diminui o aspecto genérico da rede neural.

Além da etapa de treinamento, há a etapa de testes. Por isso, os dados são separados aleatoriamente entre dados para treinamento e dados para testes, assim a rede neural pode ser validada. Portanto a análise usando uma rede neural pode ser útil para este projeto principalmente por causa da sua pequena demanda computacional. Além disso, um número alto de dados foi obtido. Em um primeiro momento será treinada uma rede neural para prever a capacidade de uma bateria ou o $\mathrm{SOH}$ de acordo com a resistência e a capacitância do modelo de Randle. 


\subsection{Lógica fuzzy}

\subsubsection{Base teórica da lógica fuzzy}

Salkind et al (1999) explica que a lógica fuzzy possibilita a modelagem de sistemas não lineares sem a necessidade de um modelo matemático explicito. $O$ exemplo clássico de lógica fuzzy usado em várias fontes (SALKIND et al, 1999; ZENATI; DESPREZ; RAZIK, 2010; SINGH; REISNER, 2002) é o da medição da temperatura de um quarto ou ambiente. É possível usar um valor concreto (crisp), como por exemplo, a temperatura de um ambiente medida por um termômetro, ou um valor incerto (fuzzy) para definir a temperatura usando uma expressão como, por exemplo, "quente" ou "frio". Ou seja, os dados são classificados em categorias (fuzzy subsets), e o quanto cada dado pertence a cada categoria é medido por uma "função de pertencimento" (membership function). Segundo Lu et al (2013), a lógica fuzzy tenta simular o pensamento e tomadas de decisões dos seres humanos, algo próximo da intuição. Uma vantagem do método é a sua simplicidade apesar de necessitar conhecimento prévio das baterias.

De acordo com Zenati, Desprez e Razik (2010), as funções de pertencimento mais comuns são as funções trapezoidal, triangular, em formato de sino e gaussiana como é mostrado na figura 43:

Figura 43 - formas de funções de pertencimento: a) trapezoidal, b) triangular, c) formato de sino e d) guassiana,
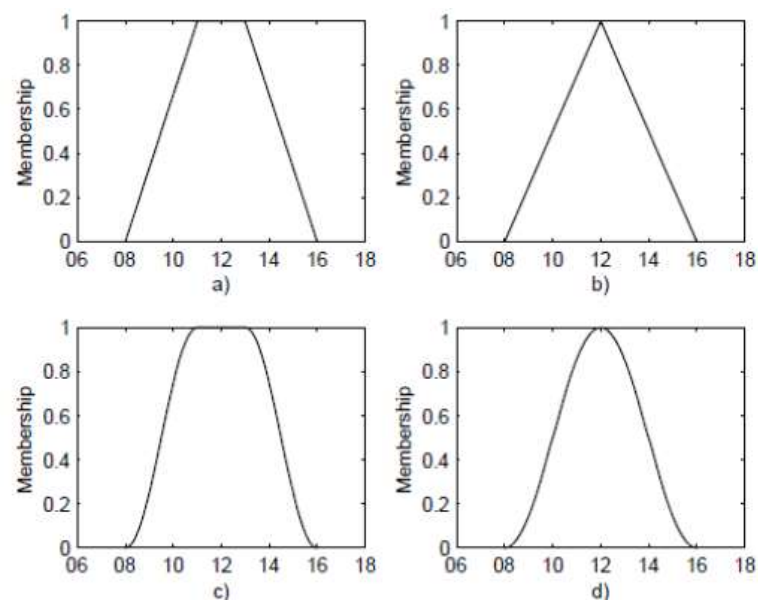

Fonte: Zenati, Desprez e Razik (2010).

A figura 44 resume os diferentes processos de um sistema baseado em lógica fuzzy. 
Figura 44 - Componentes de um sistema de lógica fuzzy,

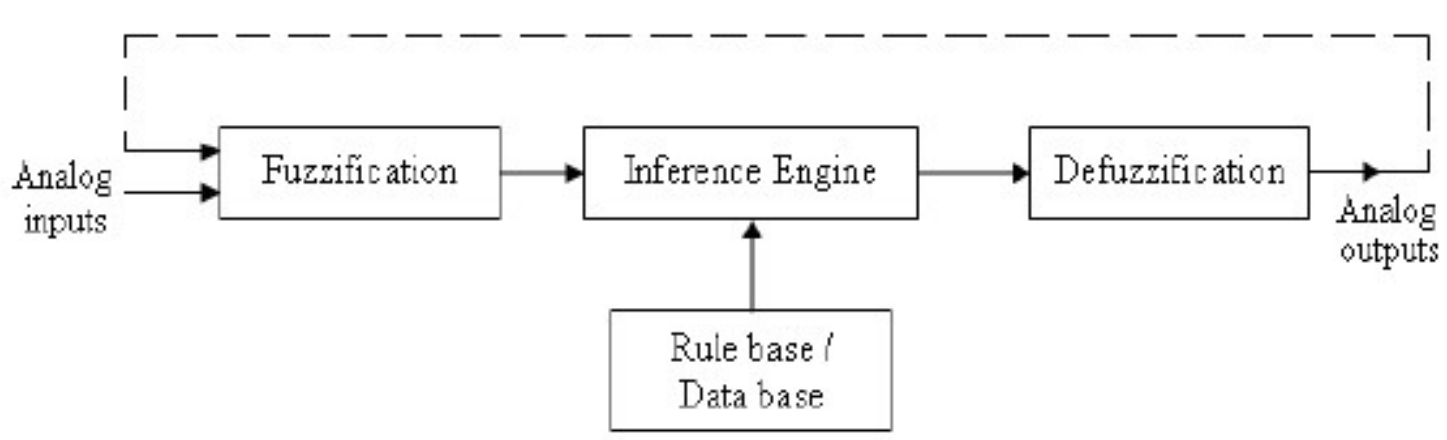

Fonte: Zenati, Desprez e Razik (2010).

Fuzificação (Fuzzification) é o processo de converter os dados analógicos (Analog inputs) em valores fuzzy usando funções de pertencimento pré-estabelecidas (ZENATI; DESPREZ; RAZIK, 2010). A segunda etapa consiste em definir o conjunto de regras (Rule base), que são baseadas no modelo "se x então y". Essas regras serão usadas no mecanismo de inferência (Inference Engine), que consiste em um método para determinar a saída dependendo dos valores de entrada. Salkind et al (1999) e Zenati, Desprez e Razik (2010) usam o modelo de Sugeno. Esse modelo consiste em achar a saída $z$ em função das entradas $x$ e y seguindo uma equação da forma $z=a x+b y+c$. A última etapa é a defuzificação (defuzzification), que transforma a saída em dados concretos (Analog Outputs), pois eles estão em forma fuzzy.

\subsubsection{Método de Sugeno de ordem 0 e 1}

Foram feitos testes com três funções de pertencimento: triangular, gaussiana e sino. As funções de pertencimento escolhidas foram a função gaussiana para o método de Sugeno de ordem 0 e a função triangular para o método de Sugeno de ordem 1 pois foram as funções de pertencimento que apresentaram melhores resultados. Nas figuras 45 a 48 são apresentados a fuzzificação dos dados de resistência e capacitância: 
Figura 45 - Funções de pertencimento triangulares para a análise da resistência.

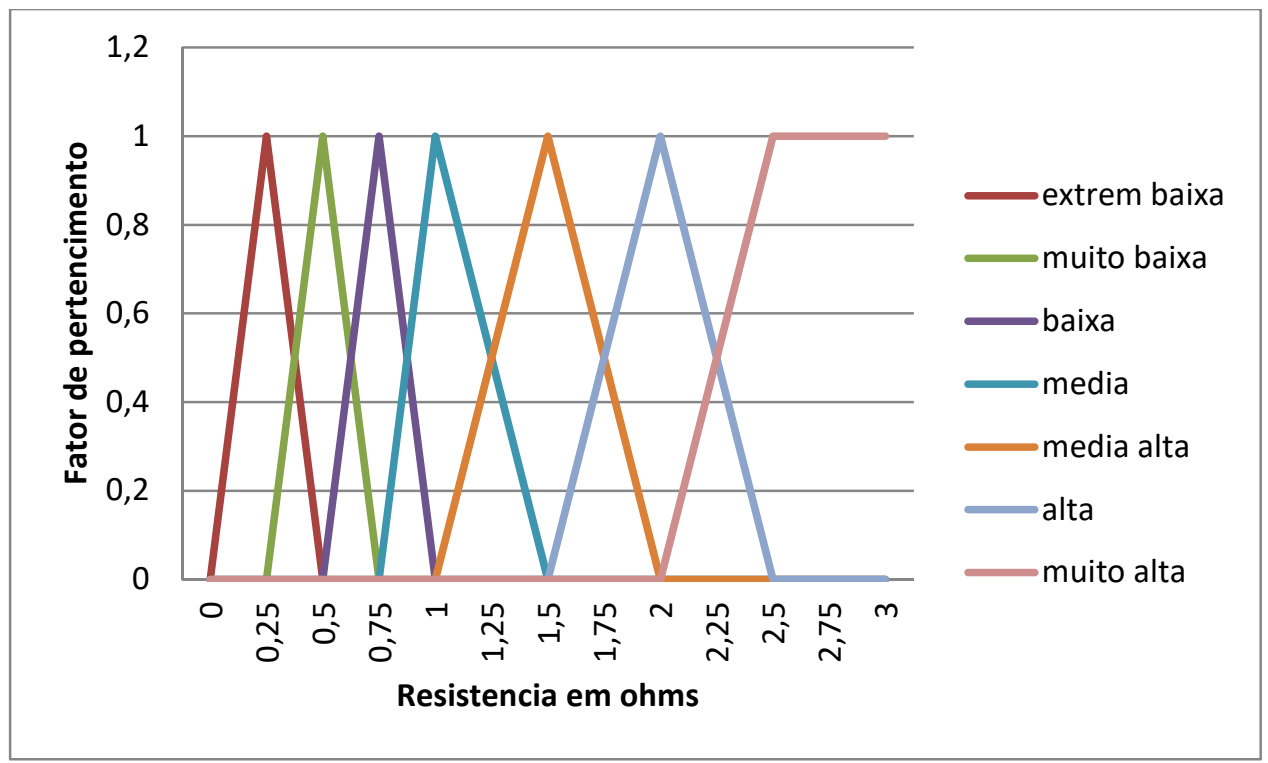

Fonte: Autor

Figura 46 - Funções de pertencimento triangulares para a análise da capacitância.

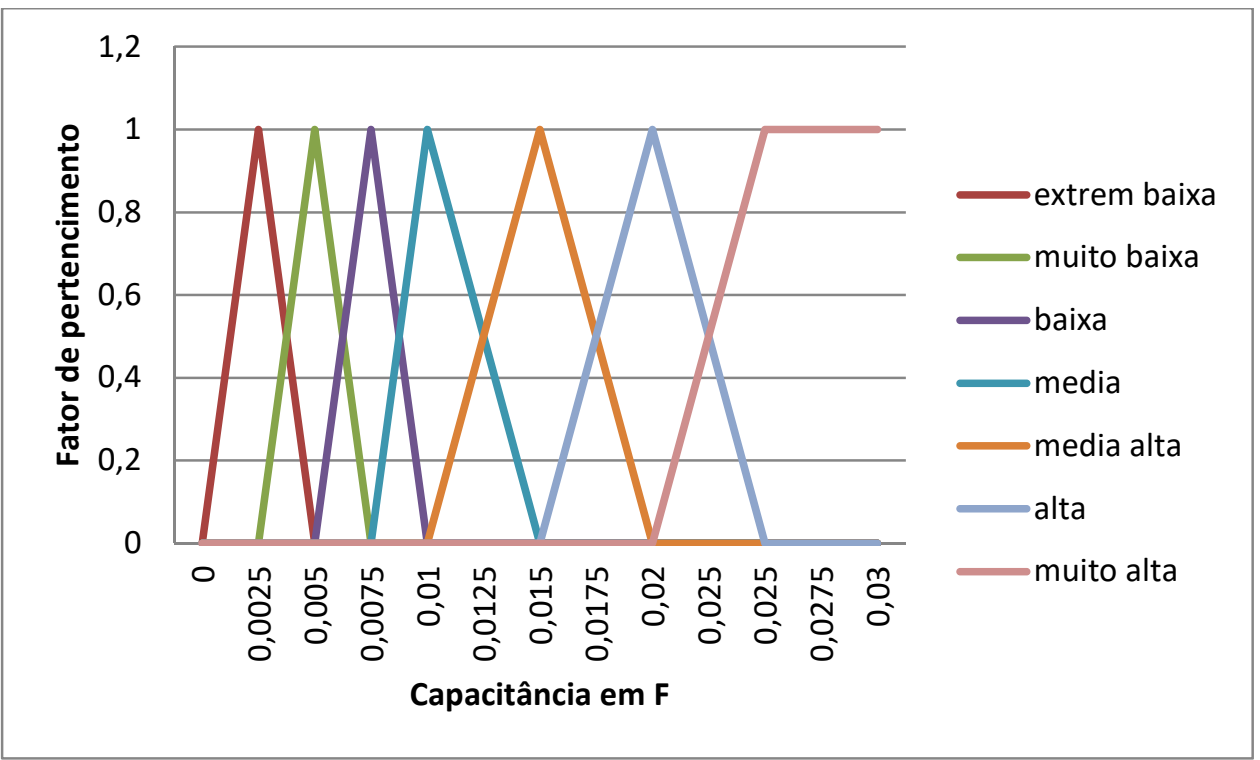

Fonte: Autor 
Figura 47 - Funções de pertencimento gaussianas para a análise da resistência.

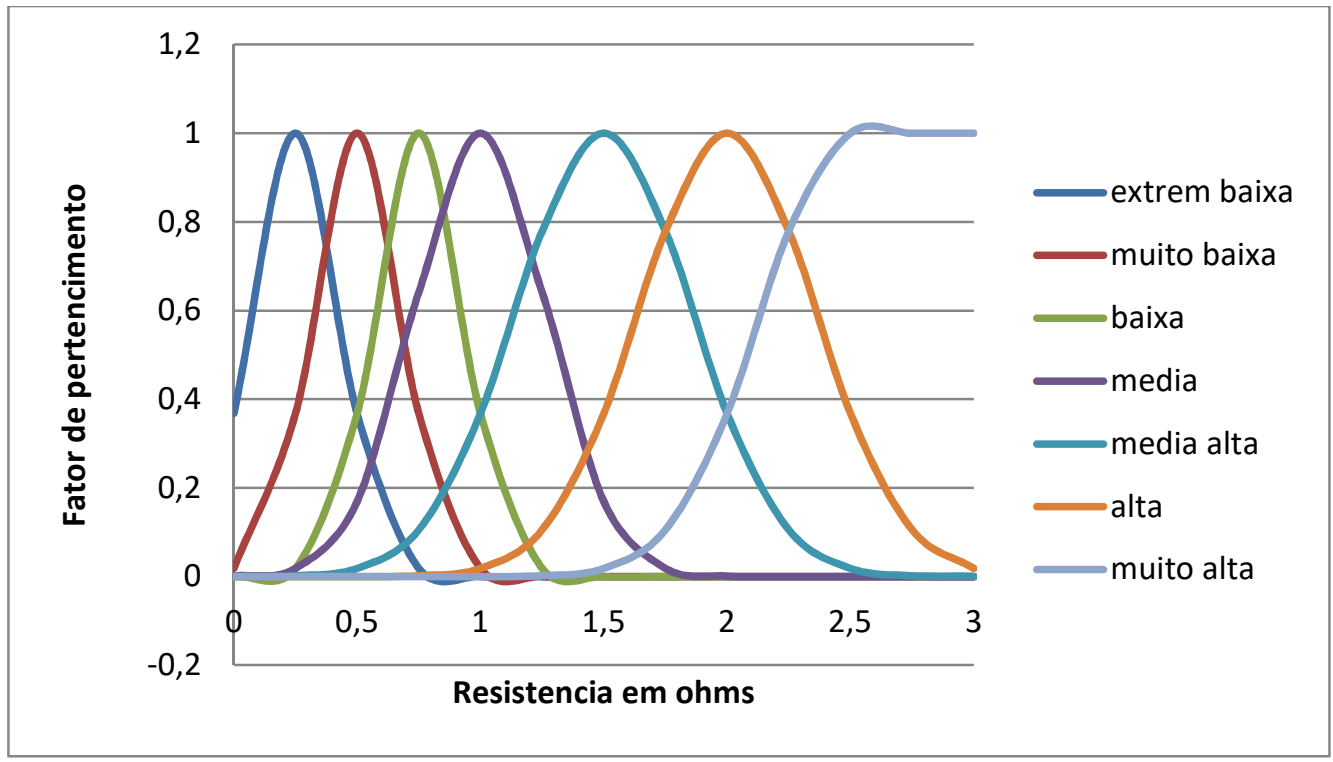

Fonte: Autor

Figura 48 - Funções de pertencimento gaussianas para a análise da capacitância.

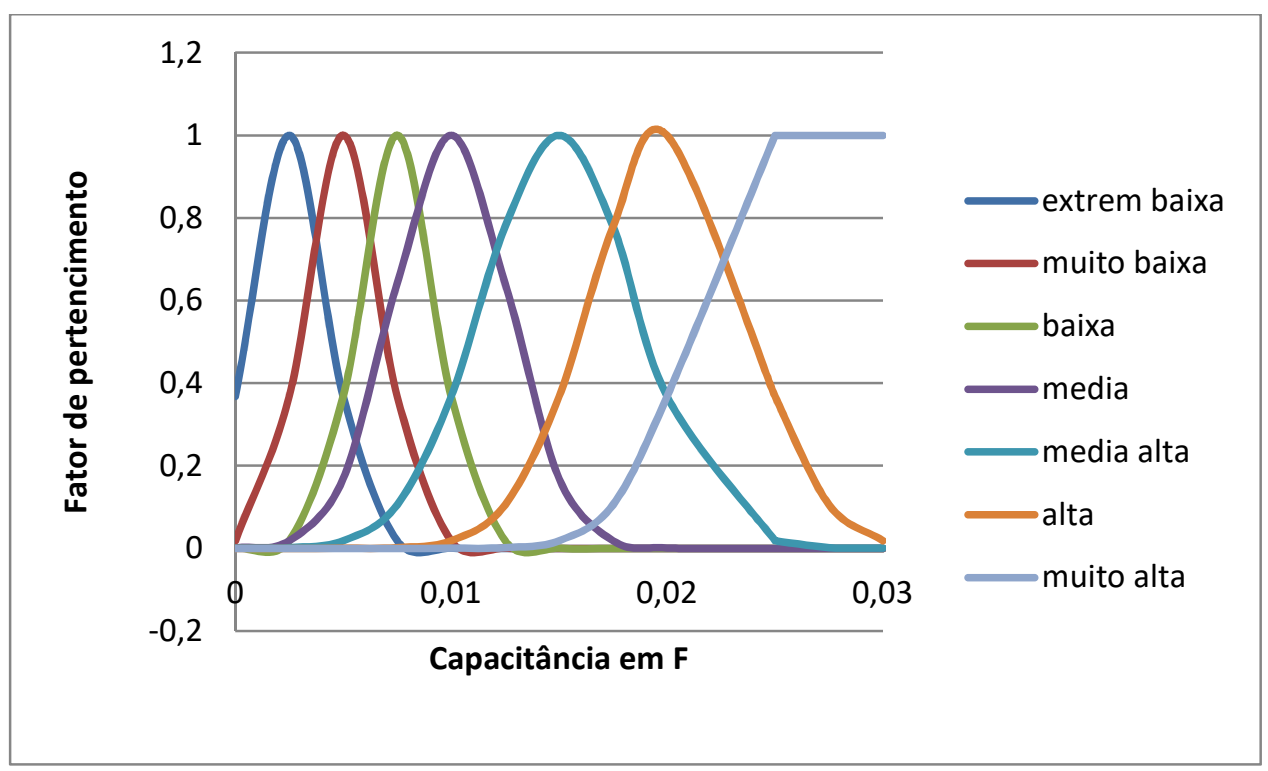

Fonte: Autor

Foram definidas 7 funções de pertencimento para cada dado de entrada: extremamente baixa, muito baixa, baixa, média, média alta, alta e muito alta.

Para cada conjunto \{resistência, capacitância\} criou-se uma regra "se x e y então z". Assim foi obtido uma tabela de regras com 49 regras. Usou-se o modelo de Sugeno 
de ordem 0 e de ordem 1 como foi descrito por Valle (2015). No modelo de ordem 0 , calculou-se o SOH de cada dado de treino usando a formula da equação 5.4.

$$
S O H=\frac{\sum_{i=1}^{49} w_{i} Q_{i}}{\sum_{i=1}^{49} w_{i}}
$$

Onde $w_{i}$ representa a ativação da regra i. Por exemplo, a ativação da regra entre as funções "extremamente baixa" da resistência e da capacitância é descrita pela formula 5.5.

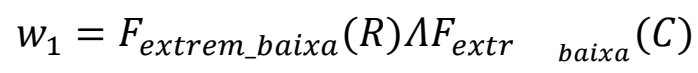

Onde $\Lambda$ representa a função mínimo. Qi é definido pela tabela de regras. No caso do modelo de Sugeno de ordem $0, Q_{i}$ é igual a uma constante.

As constantes $Q_{i}$ da tabela de regras foram atualizadas para diminuir o erro quadrático médio entre o valor de $\mathrm{SOH}$ obtido e valor medido nos dados de treino. Essa tabela de regras foi em seguida utilizada para inferir o $\mathrm{SOH}$ dos dados de teste.

No modelo de Sugeno de ordem 1 o processo é parecido. A diferença é que o SOH é calculado com a fórmula 5.6.

$$
S O H=\frac{\sum_{i=1}^{k} w_{i} f_{i}\left(x_{1}, x_{2}, \ldots, x_{n}\right)}{\sum_{i=1}^{k} w_{i}}
$$

Onde $f_{i}$ é um polinômio de ordem 1(equação 5.7).

$$
f_{1}=r_{1} * R+c_{1} * C+b_{1}
$$

Ou seja, são definidas 3 tabelas de regras para o modelo de Sugeno de ordem 1, uma tabela para cada valor de entrada (R e C) e uma tabela com constantes.

Os valores dessas tabelas de regras foram atualizados da mesma forma, ou seja, usando os dados de treino para obter o menor erro quadrático médio.

\subsubsection{Exemplos de análise de baterias usando lógica fuzzy}

Foram encontrados 4 artigos que usam lógica fuzzy em sistemas de análise de gestão de baterias. Singh e Reisner $(2002,2006)$ usam fuzzy para prever quantos pulsos a bateria de um desfibrilador aguenta. É uma previsão online do SOF da bateria com outra métrica. Salkind et al (1999) e Zenati, Desprez e Razik (2010) usam lógica fuzzy para prever o SOC e SOH de baterias. 


\section{3. $\quad$ Adaptatividade}

\subsubsection{Adaptatividade na literatura}

O conceito de adaptatividade é um conceito amplo, por isso existe na literatura várias formas de definir esse conceito.

Santos (2016) define um dispositivo adaptativo como um dispositivo que "pode ser representado por um conjunto de regras expressas em qualquer formato e um conjunto de operações associado a cada uma das regras". Com essa definição, a adaptatividade pode ser usada em diversas áreas basta que o algoritmo seja definido por um conjunto de regras. Assim as operações associadas a essas regras modificam as repostas do algoritmo.

Santos (2016) utiliza as formulações definidas por Neto (2001): o dispositivo adaptativo é composto por um dispositivo não adaptativo e um mecanismo adaptativo (NETO, 2001). Além disso para definir o dispositivo é preciso definir um conjunto de regras não adaptativas, um conjunto de regras adaptativas (tanto as regras usadas no estado k quanto as possíveis regras adaptativas), um conjunto de ações adaptativas e os mecanismos que relacionam uma ação adaptativa a uma regra adaptativa.

Canovas e Cugnasca (2016) retomam a ideia que um algoritmo adaptativo é composto por uma parte não adaptativa e dão o exemplo da Basic Adaptive Language (BADAL), uma linguagem de programação adaptativa onde a componente adaptativa é parte do código fonte, que pode ser alterado durante a execução do programa. Canovas e Cugnasca (2016) definem também o conceito de modelos adaptativos como "um modelo executável com capacidade de auto modificação ao longo de sua execução", e dividem esse modelo adaptativo em duas partes: parte adaptativa ("elementos que expressam em que situações deve ocorrer adaptatividade") e semântica adaptativa ("expressa quais são os efeitos decorrentes das ações adaptativas").

Whittle et al (2009) definem sistemas adaptativos como sistemas com a "capacidade de maneira autônoma de modificar o seu comportamento" durante a execução. Segundo Whittle et al (2009), um dos principais desafios de sistemas adaptativos é lidar com a incerteza, tanto a incerteza que deriva das entradas quanto a incerteza que deriva dos requisitos, que poderiam mudar de caso a caso. Para tratar problemas 
dessa ordem, os autores criaram uma linguagem que trata a incerteza dos requisitos da aplicação, modificando-os quando o ambiente muda.

Portanto, a adaptatividade é um conceito amplo que possui diferentes formulações e definições que dependem do problema que se deseja resolver. A criação de linguagens de programação adaptativas facilita a escolha dessas formulações, pois parte das formulações estão embutidas na criação da linguagem.

Porém a formulação escolhida em redes neurais adaptativas é um pouco diferente. Cichocki et al (1996) diminuem o tempo de convergência modificando a taxa de aprendizado de acordo com os dados de entradas. Diferente do que é feito em redes neurais não adaptativas onde é utilizada uma taxa de aprendizado fixa ou uma taxa de aprendizado que diminui com uma função pré-definida.

Amari e Cichocki (1998) fazem algo parecido ao usarem um algoritmo adaptativo para modificar o algoritmo de aprendizagem da rede neural em busca de maior eficiência estatística e menor tempo de convergência.

Lin e Lee (1991) apresentam uma solução parecida para um sistema híbrido entre rede neural e lógica fuzzy. O principal objetivo também é diminuir o tempo de convergência do algoritmo de aprendizado, o que é crítico principalmente para a inferência das regras do sistema de lógica fuzzy tradicional. Lin e Lee (1991) apresentam duas soluções hibridas e mostram que a adaptatividade do algoritmo de aprendizagem apresenta resultados melhores que o sistema tradicional.

Lin e Xu (2016) desenvolveram um sistema híbrido entre rede neural, lógica fuzzy e algoritmos genéticos. O sistema é dividido em dois componentes, um deles sendo a rede neural fuzzy que serve de entrada para o algoritmo genético. Um algoritmo adaptativo (self-clustering algorithm (SCA)) é utilizado para separar os dados em clusters. Esse algoritmo online se adapta aos valores de entrada.

O uso de adaptatividade em gerenciamento de baterias é diverso, muitas vezes usando outras terminologias. Hu et al (2015) descrevem o algoritmo usado por eles como "estimativa online", onde o método de Relevance Vector Machine (RVM) é usado para estimar e a dependência da capacidade da bateria com algumas características. 
Bai et al (2014) usam um algoritmo de "modelo livre" atualizando um modelo baseado em filtro de Kalman e redes neurais para se adaptar a novos dados obtidos da tensão da bateria e estimar o SOC e SOH.

Fleischer et al (2012) apresentam um algoritmo de "auto aprendizado" que combina diferentes algoritmos (redes neurais, lógica fuzzy e filtro de Kalman) para prever o SOF atual da bateria baseado no SOC, SOH e temperatura.

Lakkis et al (2015) desenvolvem um algoritmo com observador adaptativo não linear que se adapta tanto na carga quanto na descarga da bateria para estimar os níveis de SOC e SOH.

É possível concluir que a adaptatividade é usada em alguns momentos em redes neurais e outros algoritmos inspirados em sistemas biológicos para facilitar e simplificar o processo de aprendizado e diminuir o tempo de convergência. Em outros casos, a adaptatividade é usada em gerenciamento de baterias e inteligência artificial para melhorar os resultados do algoritmo para uma bateria específica, como é feito em outras áreas da adaptatividade.

\subsubsection{Inserção de adaptatividade}

Como foi dito antes, adaptatividade pode ser definida como um conjunto de regras modificáveis. Portanto foi inserida adaptatividade na rede neural depois da rede neural já ter os seus pesos definidos. Primeiramente, os dados de 20 baterias (1000 conjuntos de dados) foram usados para treinar e testar a rede neural, em seguida os 50 dados da bateria restante foram usados para testar o algoritmo adaptativo, mudando os pesos da rede neural (ou as regras de execução da rede neural) para que esta se adapte aos dados da última bateria, estimando com maior precisão a capacidade atual da bateria. Repetiu-se o processo 21 vezes, criando assim 21 redes neurais, uma para cada bateria, e o objetivo era atualizar esses dados de forma adaptativa e verificar que o erro médio fosse menor. Assim os resultados seriam mais confiáveis, pois não dependeriam da escolha da bateria.

Nesse processo, simulou-se a obtenção de dados que o dispositivo conectado à bateria conseguiria. Como a função da rede neural é prever a capacidade em um momento da bateria, e essa capacidade muda de tempo em tempo, o dispositivo terá que realizar diversas previsões. Assim, a vantagem de uma rede neural adaptativa é 
que as previsões ficam mais precisas para cada dado recebido. É importante notar que essa técnica só é possível em caso onde a capacidade da bateria pode ser medida de outra maneira. Por exemplo, a corrente de descarga for conhecida, é possível calcular a capacidade da bateria com o tempo de descarga. Uma aplicação que essa técnica pode ser utilizada é na medição da qualidade de baterias de desfibriladores. Quando a bateria está nova, é conhecido o número de vezes que o desfibrilador pode ser usado com uma carga cheia. Assim, um modo de calcular o $\mathrm{SOH}$ dessa bateria é o número vezes que o aparelho foi usado. Obtém-se assim a evolução do $\mathrm{SOH}$.

Portanto nesse estudo os dados possuem uma ordem definida e o objetivo será melhorar a previsão do próximo dado.

No final, o erro médio da rede neural adaptativa é comparado ao erro médio da rede neural inicial. O esperado era que a diferença entre o erro médio da rede neural inicial e o erro médio adaptativo fosse aumentando para cada dado. Porém esse aumento não é constante nem totalmente garantido.

Por isso, modificou-se o algoritmo adaptativo atualizando a rede somente quando a rede adaptativa tivesse um erro menor para o dado apresentado. Atualizou-se a rede neural com o algoritmo do gradiente decrescente, o mesmo usado para o treino da rede. A premissa é que os dados que obtiveram um erro maior na rede adaptativa são diferentes dos outros dados da bateria por razões aleatórias (temperatura ambiente, corrente de carga ou descarga, etc). Assim para obter um erro médio adaptativo menor, esse dado é ignorado na atualização da rede. Como lembram Fleischer et al (2012), o sobre ajustamento pode ser um problema para algoritmos adaptativos já que o ruído pode mudar o modelo mais que outros dados. Por isso, com um novo dado, o modelo adaptativo deve escolher se esse dado será usado ou não para atualizar o sistema. (FLEISCHER et al, 2012)

Além disso, o dispositivo pode escolher qual das duas redes (a adaptativa ou a inicial) ele utilizará para prever o próximo dado considerando qual tem um erro médio menor para os dados já adquiridos. 
Sendo assim, a premissa é que os dados mudam um pouco de bateria para bateria e que os dados de uma dada bateria são mais próximos entre si. O algoritmo busca se aproveitar dessa particularidade para obter resultados mais precisos.

Esse algoritmo é simples de ser implementado em microcontroladores, os mesmos necessários para fazer as medições da resistência e capacitância interna das baterias. Esse método é parecido com o método de aprendizado de transferência (transfer learning) descrito por Torrey e Shavlik (2000).

\subsection{Melhorando a previsão de EOL com redes neurais}

Ao usar redes neurais ou outros métodos para prever o $\mathrm{SOH}$, é possível estimar o EOL. Porém o objetivo principal do método não é estimar o EOL, portanto essa previsão não é sempre eficiente. Por exemplo, o método pode obter um erro pequeno na previsão e errar a previsão do EOL se o SOH real estiver muito próximo do EOL.

Por isso, se inspirando na técnica descrita por Matsumoto e Hernandez-del-Moral (2016), criou-se um método para melhorar a previsão do SOH e do EOL. Após a previsão do $\mathrm{SOH}$ com uma rede neural, utilizou-se uma segunda rede neural para prever se a bateria atingiu o EOL. As entradas dessa segunda rede neural são a resistência e a capacitância internas da bateria. Portanto as duas redes neurais podem obter resultados conflitantes.

Finalmente, criou-se uma terceira rede neural que trata somente os casos que tiveram esses resultados conflitantes. As entradas da rede neural são a resistência e capacitância internas, o SOH calculado pela primeira rede neural e o EOL calculado pela segunda rede neural. A saída da rede é um status. Esse status vale 0 se o $\mathrm{SOH}$ real é mais de $8 \%$ menor que o $\mathrm{SOH}$ calculado, vale 1 se o $\mathrm{SOH}$ é $8 \%$ maior que o $\mathrm{SOH}$ calculado ou vale 0,5 se o erro absoluto for menor que $8 \%$.

O objetivo dessa última rede neural é descobrir os casos que possuem um erro muito discrepante da média e corrigi-lo. Como foi feito por Matsumoto e Hernandez-delMoral (2016), o objetivo é corrigir as previsões que estão muito longe da média e assim melhorar o resultado da previsão final. 


\subsection{Comparação dos métodos}

Le e Tang (2011) definem o SOH de uma bateria em função da resistência interna ou em função da sua capacitância interna. Para medir a eficácia dos diferentes métodos usados, os resultados obtidos foram comparados com um método linear.

Como foi dito anteriormente, a resistência interna e a capacitância variam devido a vários fatores, não somente o $\mathrm{SOH}$. Assim analisou-se também a relação entre essas medidas e o SOH como é feito por Liu et al (2013).

A reta com o menor erro médio quadrático para essas duas variáveis é calculada. Essa é uma forma simples de estimar o $\mathrm{SOH}$ somente com a informação da resistência interna ou da capacitância interna.

Assim é possível quantificar a qualidade da análise por outros métodos comparando com uma análise mais simples de ser realizada. Essa análise estatística é feita com os mesmos dados.

\subsection{Validação cruzada}

Em todos os métodos foi utilizada a técnica de dez interações de validação cruzada (ten fold cross validation). Nessa técnica, divide-se o conjunto de dados em dez. Para cada interação, o sistema é treinado com nove décimos dos dados e os dados restantes servem como teste. O processo é repetido dez vezes, ou seja, sempre há um décimo dos dados que serve como conjunto de teste. No final são calculados o erro médio de cada conjunto de teste e o erro médio de todos os dados. A estimativa do processo com k interações de validação cruzada não é tendenciosa, pois os k conjuntos de dados são escolhidos aleatoriamente (KOHAVI, 1995). 


\section{Resultados}

\subsection{Redes neurais}

Foi desenvolvido um programa para computador em linguagem $\mathrm{C}++$ que treinasse $\mathrm{e}$ testasse a rede neural e algumas topologias foram testadas. $O$ código desse software está no apêndice B. A topologia que obteve melhores resultados foi escolhida. A rede neural criada possui duas camadas escondidas, a primeira com 9 neurônios e a segunda com 5 neurônios. A topologia dessa rede pode ser vista na figura 49 , que foi criada no software MBP (LOPES; RIBEIRO, 2011).

Figura 49 - Topologia da rede neural.

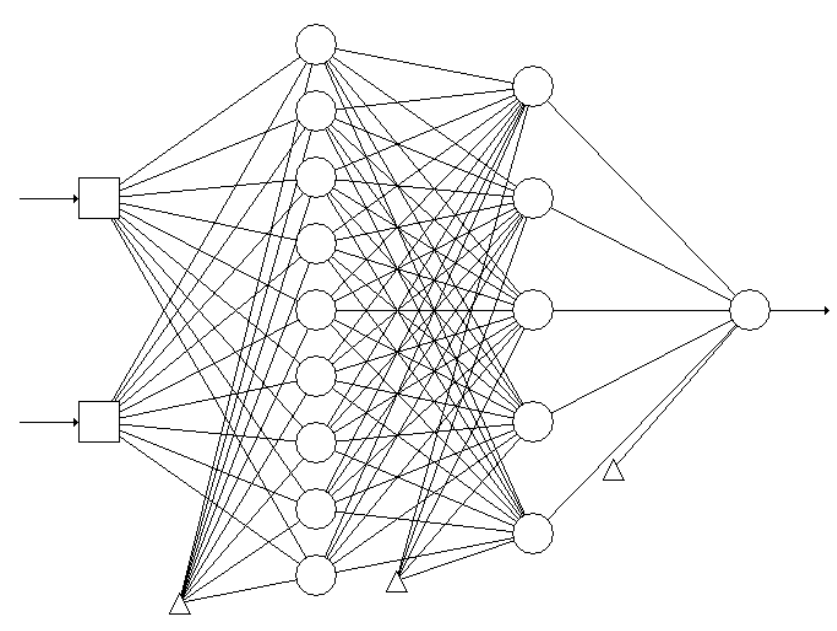

Fonte: Autor.

Todas as funções da rede neural são sigmoides. Os pesos finais obtidos estão indicados nos apêndices. 
Foram obtidos os resultados descritos na tabela 4 para a rede neural.

Tabela 4 - Resultados da rede neural.

\begin{tabular}{|c|c|}
\hline Erro médio do treino $\mathrm{n}$ 0 & 7,376 \\
\hline Erro médio do treino $\mathrm{n}$ 1 & 7,432 \\
\hline Erro médio do treino $\mathrm{n}$ 2 & 7,295 \\
\hline Erro médio do treino $\mathrm{n}$ 3 & 6,89 \\
\hline Erro médio do treino $\mathrm{n}$ 4 & 6,369 \\
\hline Erro médio do treino $\mathrm{n}$ 5 & 6,439 \\
\hline Erro médio do treino $\mathrm{n}$ 6 & 7,165 \\
\hline Erro médio do treino $\mathrm{n}$ 7 & 7,127 \\
\hline Erro médio do treino $\mathrm{n}$ 8 & 6,562 \\
\hline Erro médio do treino $\mathrm{n}$ 9 & 6,954 \\
\hline Erro médio de todos os dados & 6,958 \\
\hline
\end{tabular}

Fonte: Autor

Os valores dos erros estão em porcentagem do SOH. Percebeu-se que o erro é maior para os dados onde o SOH é menor, pois depois de certa capacidade a bateria fica muito instável. É possível visualizar isso na figura 50 , onde se calculou o erro médio por faixa de $\mathrm{SOH}$ :

Figura 50 - Gráfico do erro médio da rede neural por faixa de valor de $\mathrm{SOH}$.

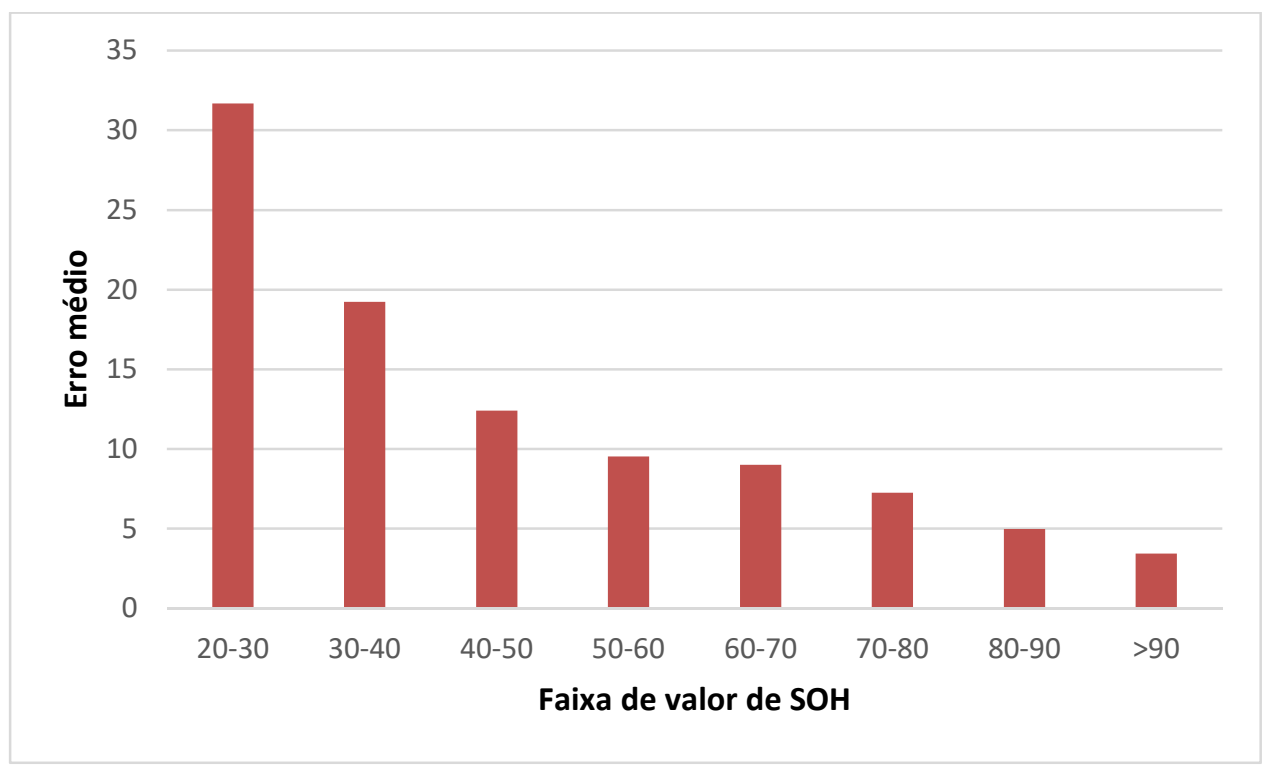

Fonte: Autor

Para baterias com capacidade maior que $80 \%$ o erro é inferior a $5 \%$. Essas baterias representam a maioria dos dados de teste. Para uma aplicação de baterias estacionarias esse dado pode ser útil, mas é importante que o sistema identifique 
baterias que chegaram ao fim da vida (EOL). Portanto calculou-se o número de diagnósticos errados de EOL que o sistema teria. Considerou-se que toda bateria que tinha SOH menor que o EOL estava no fim da vida. Definiu-se que houve erro de EOL quando o sistema calculou um $\mathrm{SOH}$ com erro maior que $5 \%$ e o status correspondente do EOL estava errado. Isso foi feito para que erros pequenos de $\mathrm{SOH}$ próximos do EOL não gerassem erros de EOL. O número de erros de EOL para a rede neural é apresentado na tabela 5 .

Tabela 5 - Resultados da rede neural.

\begin{tabular}{|c|c|c|c|}
\hline Intervalo & $\begin{array}{c}\text { Número de } \\
\text { medidas }\end{array}$ & $\begin{array}{c}\text { Erro médio } \\
\text { treino }\end{array}$ & $\begin{array}{c}\text { Número de erro } \\
\text { EOL }\end{array}$ \\
\hline $20-30$ & 17 & 31,676 & 3 \\
\hline $30-40$ & 36 & 19,230 & 1 \\
\hline $40-50$ & 56 & 12,424 & 1 \\
\hline $50-60$ & 60 & 9,542 & 3 \\
\hline $60-70$ & 100 & 9,018 & 15 \\
\hline $70-80$ & 190 & 7,251 & 59 \\
\hline $80-90$ & 317 & 4,990 & 55 \\
\hline$>90$ & 274 & 3,450 & 4 \\
\hline Total & 1050 & 6,958 & 141 \\
\hline
\end{tabular}

Fonte: Autor

\subsection{Lógica Fuzzy}

Um software foi criado para analisar com lógica fuzzy os dados. O código desse software está no apêndice $C$.

\subsubsection{Resultados para o modelo de Sugeno de ordem 0}

No modelo de Sugeno de ordem 0 usou-se funções de pertinência gaussianas tanto para a resistência quanto para a capacitância. A regra de ativação usada foi a função mínimo (equação 5.5). A tabela de regras obtida para o modelo de Sugeno de ordem 0 pode ser visto nos apêndices. 
Os resultados da tabela 6 foram obtidos para o modelo de Sugeno de ordem 0:

Tabela 6 - Resultados do modelo de Sugeno de ordem 0.

\begin{tabular}{|c|c|}
\hline Erro médio do treino $\mathrm{n} 0$ & 7,397 \\
\hline Erro médio do treino $\mathrm{n} 1$ & 7,388 \\
\hline Erro médio do treino $\mathrm{n} 2$ & 7,189 \\
\hline Erro médio do treino $\mathrm{n} 3$ & 6,906 \\
\hline Erro médio do treino $\mathrm{n} 4$ & 6,966 \\
\hline Erro médio do treino $\mathrm{n} 5$ & 6,392 \\
\hline Erro médio do treino $\mathrm{n} 6$ & 7,144 \\
\hline Erro médio do treino $\mathrm{n} 7$ & 7,6 \\
\hline Erro médio do treino $\mathrm{n} 8$ & 6,691 \\
\hline Erro médio do treino $\mathrm{n} 9$ & 6,871 \\
\hline Erro médio de todos os dados & 7,053 \\
\hline
\end{tabular}

Como nos outros modelos, percebeu-se que o erro médio é maior quanto menor é o $\mathrm{SOH}$, como é mostrado na figura 51 :

Figura 51 - Gráfico do erro médio no modelo de Sugeno de ordem 0 por faixa de valor de SOH.

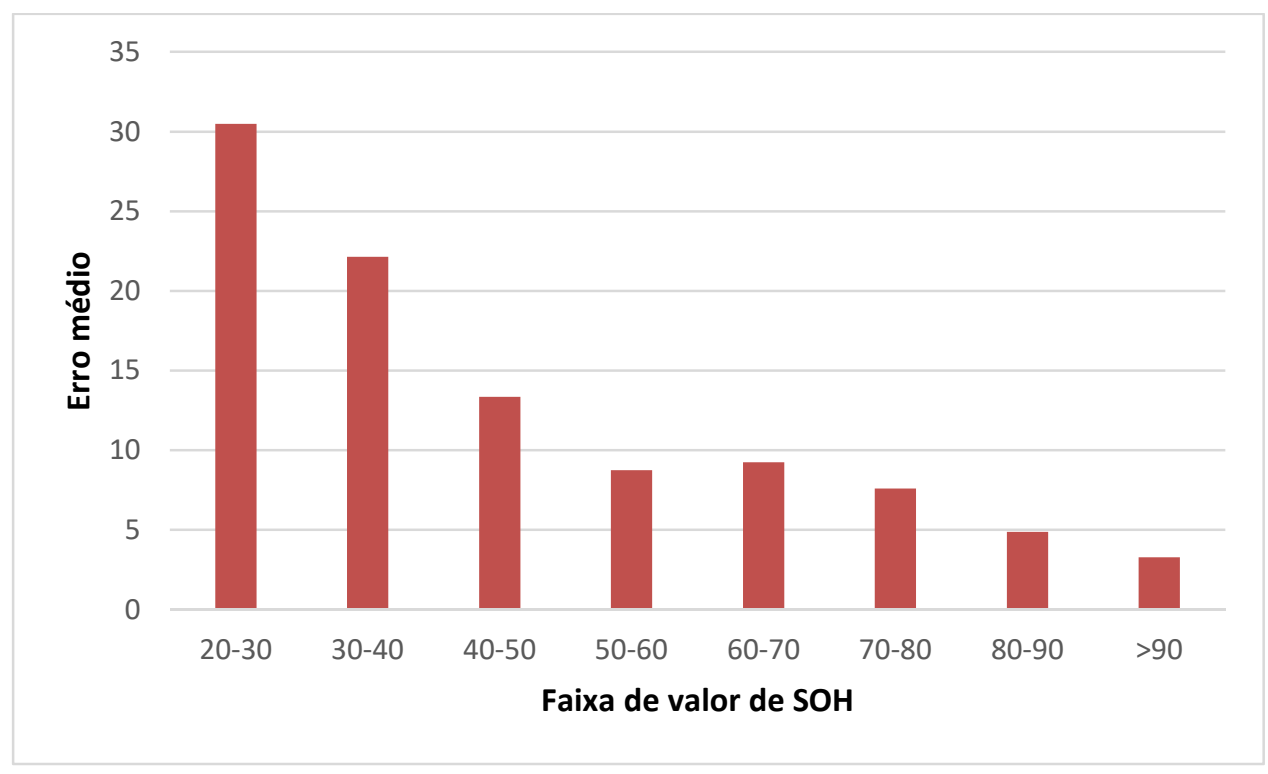

Fonte: Autor

Como nos outros modelos a análise do EOL foi realizada. No modelo de ordem 0 obteve-se os resultados da tabela 7 . 
Tabela 7 - Resultados do modelo de Sugeno de ordem 0.

\begin{tabular}{|c|c|c|c|}
\hline Intervalo & $\begin{array}{c}\text { Número de } \\
\text { medidas }\end{array}$ & $\begin{array}{c}\text { Erro médio } \\
\text { treino }\end{array}$ & $\begin{array}{c}\text { Número de erro } \\
\text { EOL }\end{array}$ \\
\hline $20-30$ & 17 & 30,489 & 3 \\
\hline $30-40$ & 36 & 22,150 & 1 \\
\hline $40-50$ & 56 & 13,360 & 1 \\
\hline $50-60$ & 60 & 8,761 & 3 \\
\hline $60-70$ & 100 & 9,256 & 16 \\
\hline $70-80$ & 190 & 7,595 & 61 \\
\hline $80-90$ & 317 & 4,881 & 59 \\
\hline$>90$ & 274 & 3,286 & 3 \\
\hline Total & 1050 & 7,053 & 147 \\
\hline
\end{tabular}

Fonte: Autor

É possível observar que a proporção de erro de EOL é pequena o suficiente para a aplicação já que a deterioração da bateria ocorreria em um longo período de tempo, portanto várias medidas poderiam ser realizadas e as medidas incorretas seriam excluídas. Além disso, as baterias com um maior erro estão com um $\mathrm{SOH}$ muito pequeno, então o sistema já teria identificado como EOL em uma medida anterior.

\subsubsection{Resultados para o modelo de Sugeno de ordem 1}

No modelo de Sugeno de ordem 1 usou-se funções de pertinência triangulares tanto para a resistência quanto para a capacitância. A regra de ativação usada foi a função mínimo (equação 5.5). As tabelas de regras obtidas para o modelo de Sugeno de ordem 1 são apresentadas nos apêndices.

Os resultados obtidos para o modelo de Sugeno de ordem 1 estão na tabela 8. 
Tabela 8 - Resultados do modelo de Sugeno de ordem 1.

\begin{tabular}{|c|c|}
\hline Erro médio do treino $\mathrm{n}$ 0 & 7,586 \\
\hline Erro médio do treino $\mathrm{n}$ 1 & 7,705 \\
\hline Erro médio do treino $\mathrm{n}$ 2 & 7,421 \\
\hline Erro médio do treino $\mathrm{n}$ 3 & 7,357 \\
\hline Erro médio do treino $\mathrm{n}$ 4 & 6,715 \\
\hline Erro médio do treino $\mathrm{n}$ 5 & 7,291 \\
\hline Erro médio do treino $\mathrm{n}$ 6 & 7,349 \\
\hline Erro médio do treino $\mathrm{n}$ 7 & 7,47 \\
\hline Erro médio do treino $\mathrm{n}$ 8 & 6,603 \\
\hline Erro médio do treino $\mathrm{n}$ 9 & 6,976 \\
\hline Erro médio de todos os dados & 6,818 \\
\hline
\end{tabular}

Fonte: Autor

Como no modelo anterior, o erro foi maior para $\mathrm{SOH}$ menores como é observado na figura 52.

Figura 52 - Gráfico do erro médio no modelo de Sugeno de ordem 1 por faixa de valor de SOH.

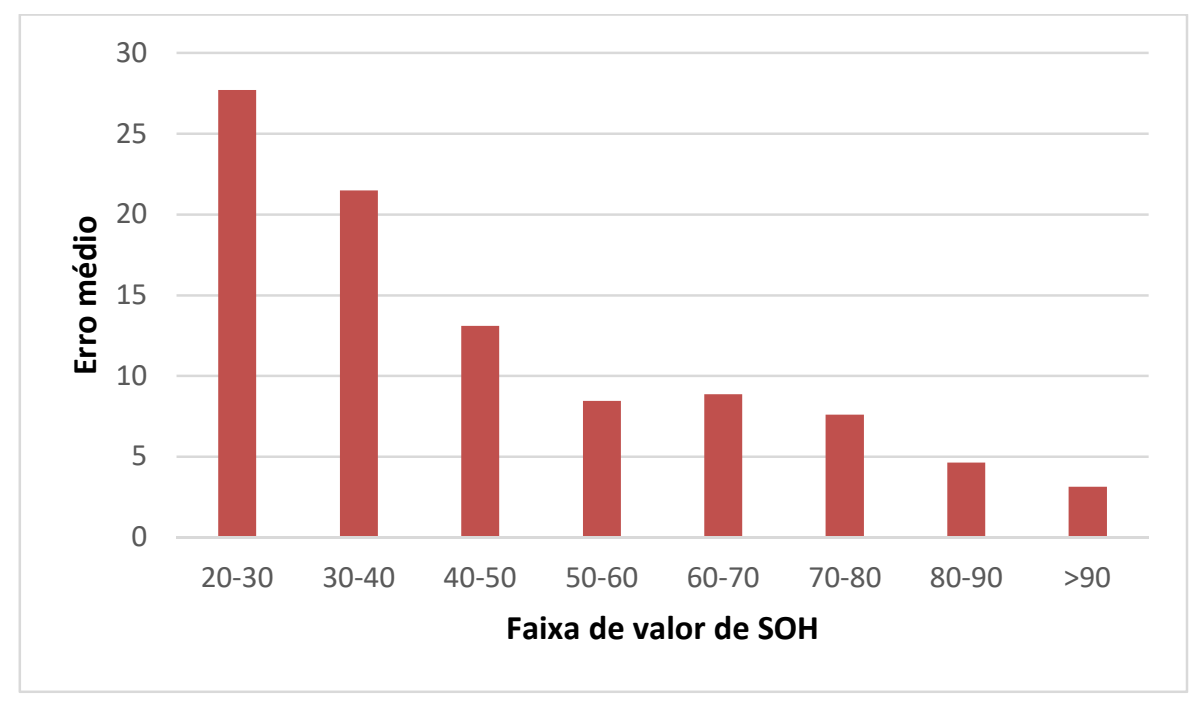

Fonte: Autor

É perceptível que o modelo de Sugeno de ordem 1 tem resultados parecidos com o de ordem 0 e que a proporção de erro de EOL continua pequena e suficiente para a aplicação como visto na tabela 9 . 
Tabela 9 - Resultados do modelo de Sugeno de ordem 0.

\begin{tabular}{|c|c|c|c|}
\hline Intervalo & $\begin{array}{c}\text { Número de } \\
\text { medidas }\end{array}$ & $\begin{array}{c}\text { Erro médio } \\
\text { treino }\end{array}$ & $\begin{array}{c}\text { Número de erro } \\
\text { EOL }\end{array}$ \\
\hline $20-30$ & 17 & 27,695 & 2 \\
\hline $30-40$ & 36 & 21,483 & 1 \\
\hline $40-50$ & 56 & 13,098 & 1 \\
\hline $50-60$ & 60 & 8,4699 & 2 \\
\hline $60-70$ & 100 & 8,869 & 16 \\
\hline $70-80$ & 190 & 7,613 & 61 \\
\hline $80-90$ & 317 & 4,654 & 64 \\
\hline$>90$ & 274 & 3,154 & 3 \\
\hline Total & 1050 & 6,818 & 150 \\
\hline & \multicolumn{2}{r|}{ Fonte: Autor } \\
\hline
\end{tabular}

Apesar do modelo de Sugeno de ordem 1 ter um número maior de erros de EOL, o erro médio do $\mathrm{SOH}$ previsto é menor. Apesar disso, o método é mais complexo que o Sugeno de ordem 0 . A escolha entre o método de ordem 0 e o método de ordem 1 depende da aplicação já que o método de ordem 1 precisa de mais memória e mais tempo para ser computado.

\subsection{Método adaptativo}

\subsubsection{Primeira versão do algoritmo adaptativo}

Em um primeiro passo, criou-se o algoritmo adaptativo que atualizava os pesos da rede neural treinada para cada dado obtido. O código desse software está no apêndice D. Na tabela 10 são apresentados os resultados dos erros médios de cada rede neural para os 50 dados de cada bateria. 
Tabela 10 - Erros médios da rede inicial e da rede adaptativa.

\begin{tabular}{|c|c|c|c|}
\hline Bateria & $\begin{array}{c}\text { Erro médio para os } 50 \\
\text { dados }\end{array}$ & $\begin{array}{c}\text { Erro médio adaptativo para os } 50 \\
\text { dados }\end{array}$ & $\begin{array}{c}\text { Diminuição em } \\
\%\end{array}$ \\
\hline 1 & 6,224 & 5,62 & 9,704 \\
\hline 2 & 1,837 & 1,855 & $-1,005$ \\
\hline 3 & 6,392 & 4,359 & 31,806 \\
\hline 4 & 6,535 & 6,026 & 7,795 \\
\hline 5 & 10,603 & 9,886 & 6,768 \\
\hline 6 & 8,497 & 5,655 & 33,45 \\
\hline 7 & 8,708 & 9,055 & $-3,826$ \\
\hline 8 & 5,991 & 5,672 & 5,328 \\
\hline 9 & 8,808 & 5,347 & 39,293 \\
\hline 10 & 6,734 & 6,472 & 3,886 \\
\hline 11 & 3,163 & 3,277 & $-3,454$ \\
\hline 12 & 5,495 & 4,634 & 15,665 \\
\hline 13 & 5,726 & 6,517 & $-12,137$ \\
\hline 14 & 7,32 & 6,324 & 13,607 \\
\hline 15 & 8,901 & 5,275 & 40,737 \\
\hline 16 & 8,281 & 6,002 & 27,526 \\
\hline 17 & 10,599 & 12,751 & $-16,876$ \\
\hline 18 & 15,268 & 9,769 & 36,015 \\
\hline 19 & 10,209 & 10,13 & 0,78 \\
\hline 20 & 8,059 & 7,808 & 3,106 \\
\hline 21 & 8,906 & 10,122 & $-12,011$ \\
\hline Média & 7,727 & 6,788 & 12,143 \\
\hline
\end{tabular}

Fonte: Autor

É possível ver na tabela 10 que algumas redes adaptativas possuem resultados piores que a rede neural inicial. A rede da bateria 17, por exemplo, tem um aumento do erro médio da ordem de $16,9 \%$. Entretanto a média de diminuição do erro é alta $(12,14 \%)$ e mostra que com poucos dados já é possível especializar a rede neural para a determinada bateria.

Outros resultados importantes são as evoluções do erro médio da rede neural adaptativa em comparação com o erro médio da rede inicial. Essa análise permitiu modificar o algoritmo adaptativo para otimizá-lo.

Era esperado que a diminuição do erro médio seguisse uma curva parecida à curva da bateria 3, que pode ser vista na figura 53. 
Figura 53 - Diminuição do erro médio da bateria 3 para cada dado novo.

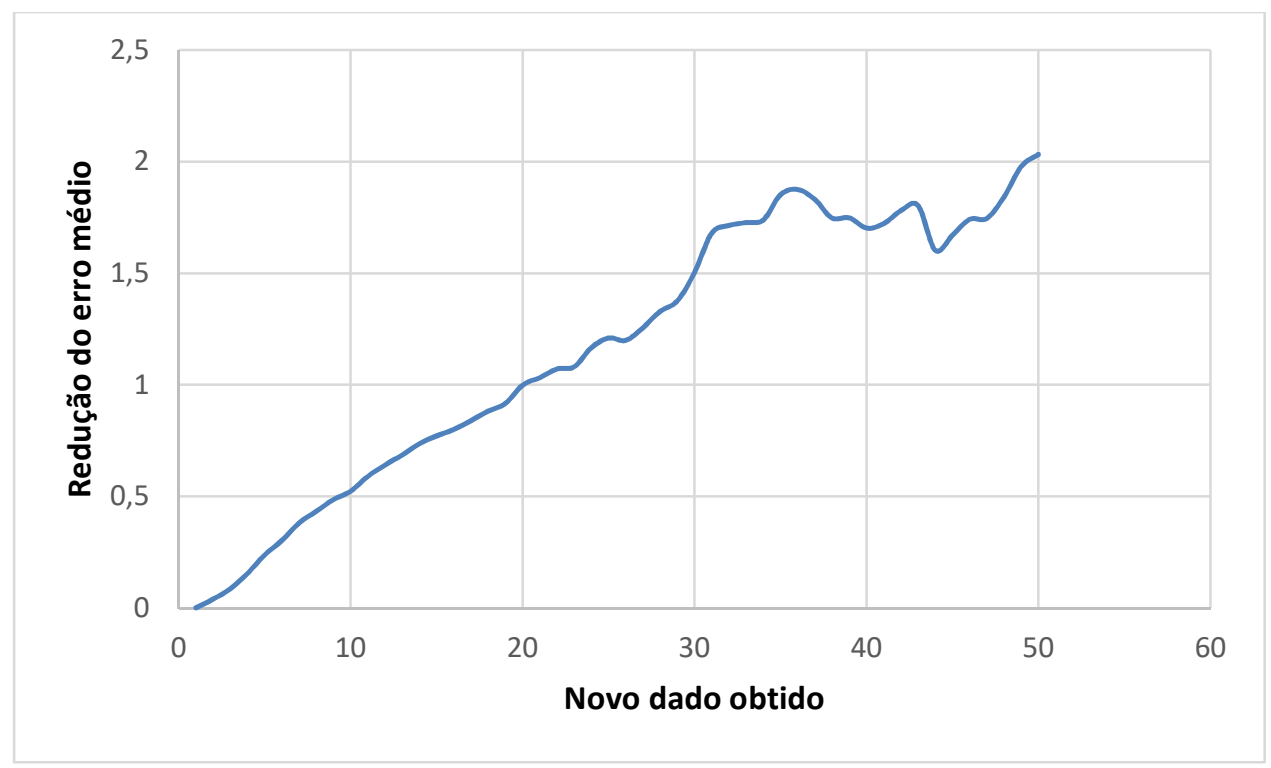

Fonte: Autor

A curva é em sua maior parte crescente monótona, indicando que o erro médio diminuiu constantemente. Porém não é o que foi visto na maioria dos casos. Um caso que pode ilustrar isso é o da bateria 5, como é visto na figura 54.

Figura 54 - Diminuição do erro médio da bateria 5 para cada dado novo.

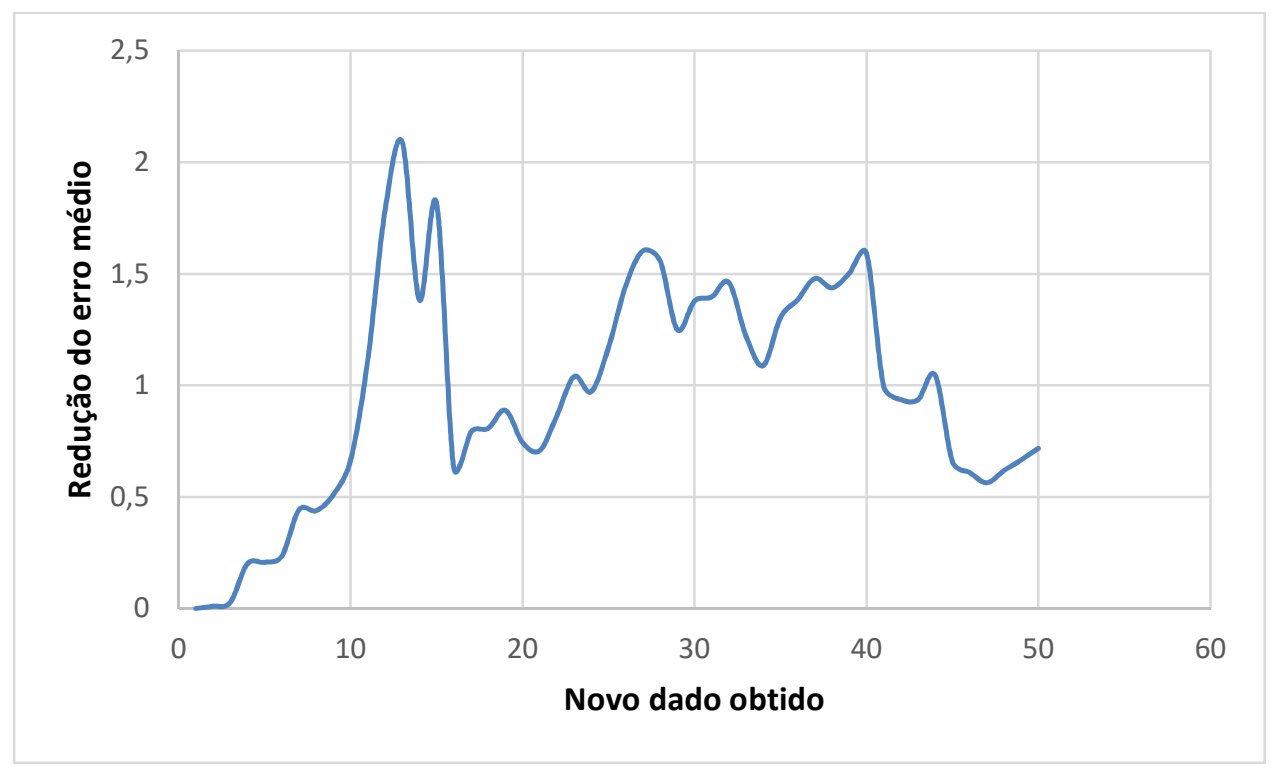

Fonte: Autor

Apesar do erro médio adaptativo sempre ser menor que o erro médio inicial, já que a curva sempre é positiva, a curva tem diversos pontos de inflexão. Ou seja, alguns 
dados aumentam o erro médio adaptativo e são melhores tratados pela rede neural inicial. Assim, as últimas previsões não são as mais precisas do conjunto de dado. Claramente há espaço para otimizar esse algoritmo.

Outros exemplos que mostram um ponto para aperfeiçoar essa primeira versão do algoritmo são os das baterias 11 e 17 (as figuras 55 e 56 respectivamente).

Figura 55 - Diminuição do erro médio da bateria 11 para cada dado novo.

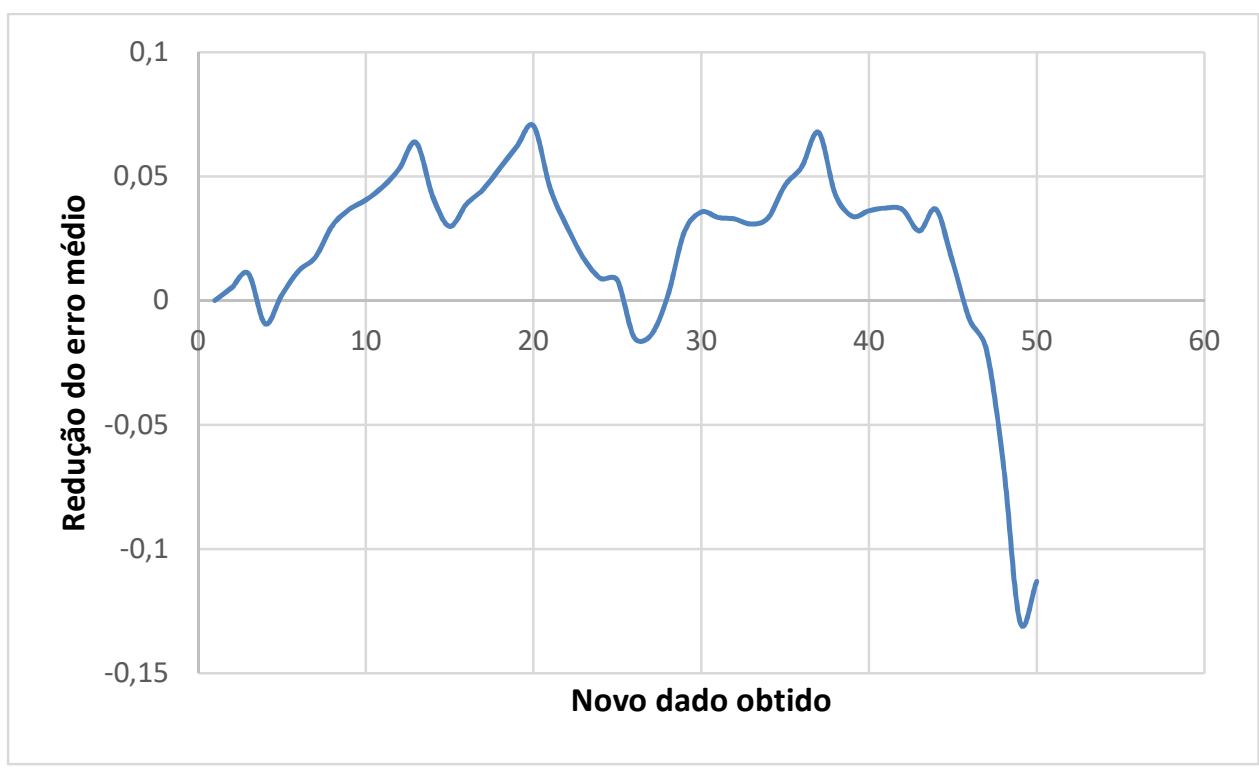

Fonte: Autor

Figura 56 - Diminuição do erro médio da bateria 17 para cada dado novo.

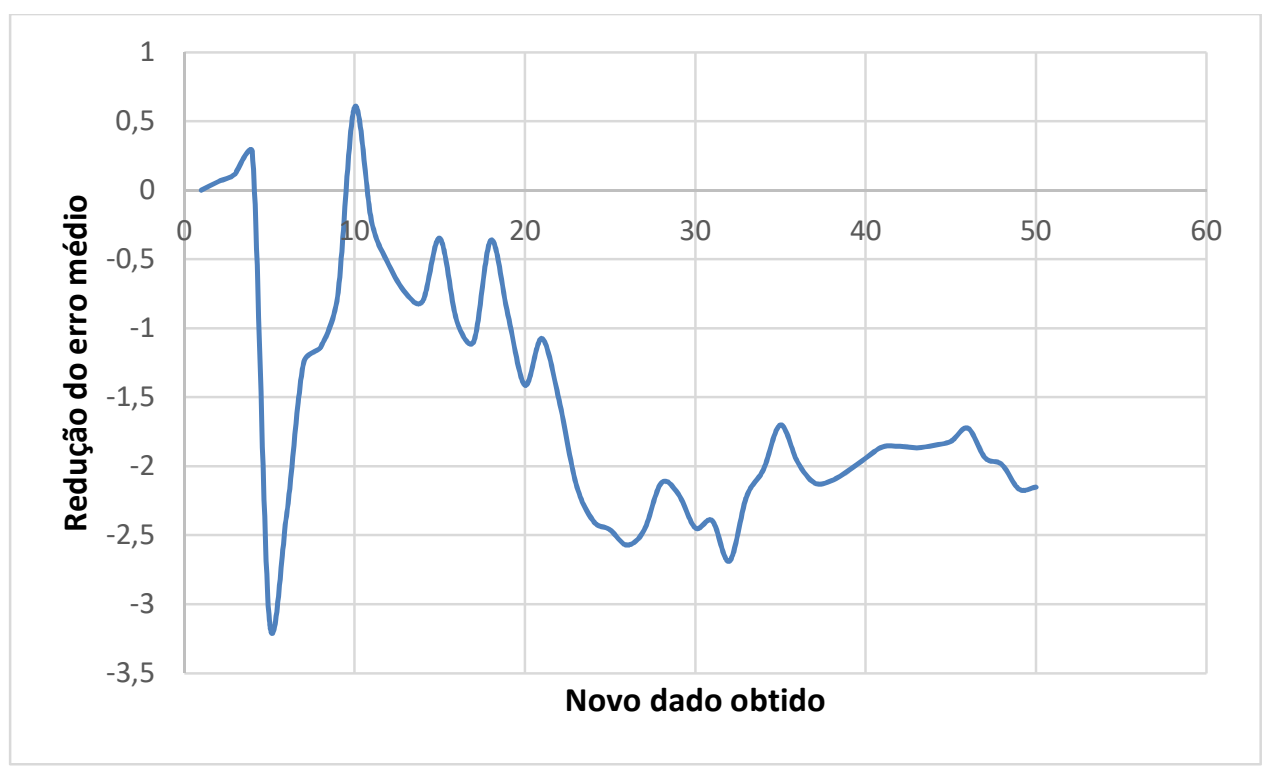

Fonte: Autor 
$\mathrm{Na}$ bateria 17, o erro médio da rede adaptativa foi quase sempre maior que o erro médio da rede inicial, portanto a adaptatividade não melhorou o resultado e poderia ser evitado. Para a bateria 11, o caso ilustra melhor o problema desse algoritmo adaptativo inicial. $O$ erro médio adaptativo foi em quase todo o teste menor que o erro da rede inicial, porém os últimos dados atrapalharam os resultados e poderiam ser excluídos.

Por isso modificou-se o algoritmo descrito anteriormente para aproveitar somente os dados que possuem melhor relação entre si.

\subsubsection{Resultados da versão final do algoritmo adaptativo}

Essa segunda versão do algoritmo não atualiza a rede neural adaptativa quando o novo dado diminuiu a diferença entre o erro médio da rede inicial com o erro médio da rede alternativa. Ou seja, toda vez que há uma queda na curva da diferença dos erros. O objetivo é que as curvas tenham uma forma mais parecida com a curva da bateria 3 da figura 53.

A tabela 11 apresenta os resultados dessa segunda versão do algoritmo e houve uma melhora em comparação com a primeira versão. 
Tabela 11 - Erros médios da rede inicial e da segunda versão da rede adaptativa.

\begin{tabular}{|c|c|c|c|}
\hline Bateria & Erro médio para os 50 dados & Erro médio adaptativo para os 50 dados & Diminuição em \% \\
\hline 1 & 6,786 & 6,383 & 5,951 \\
\hline 2 & 2,061 & 2,518 & $-18,152$ \\
\hline 3 & 6,222 & 4,318 & 30,6 \\
\hline 4 & 6,455 & 5,925 & 8,213 \\
\hline 5 & 10,646 & 8,249 & 22,519 \\
\hline 6 & 8,875 & 5,665 & 36,166 \\
\hline 7 & 8,267 & 8,816 & $-6,227$ \\
\hline 8 & 7,067 & 6,104 & 13,62 \\
\hline 9 & 9,047 & 5,749 & 36,447 \\
\hline 10 & 7,556 & 8,861 & $-14,721$ \\
\hline 11 & 2,676 & 2,797 & $-4,339$ \\
\hline 12 & 5,614 & 4,351 & 22,505 \\
\hline 13 & 6,705 & 7,077 & $-5,252$ \\
\hline 14 & 7,543 & 6,909 & 8,4 \\
\hline 15 & 8,886 & 4,429 & 50,16 \\
\hline 16 & 7,441 & 5,492 & 26,192 \\
\hline 17 & 10,243 & 11,565 & $-11,424$ \\
\hline 18 & 15,627 & 10,573 & 32,344 \\
\hline 19 & 10,269 & 10,551 & $-2,668$ \\
\hline 20 & 8,263 & 8,295 & $-0,377$ \\
\hline 21 & 9,882 & 10,652 & $-7,23$ \\
\hline Média & 7,911 & 6,918 & 12,553 \\
\hline & & & \\
\hline
\end{tabular}

Fonte: Autor

A curva da bateria 3 na figura 57 continuou com forma parecida.

Figura 57 - Diminuição do erro médio da bateria 3 na segunda versão do algoritmo.

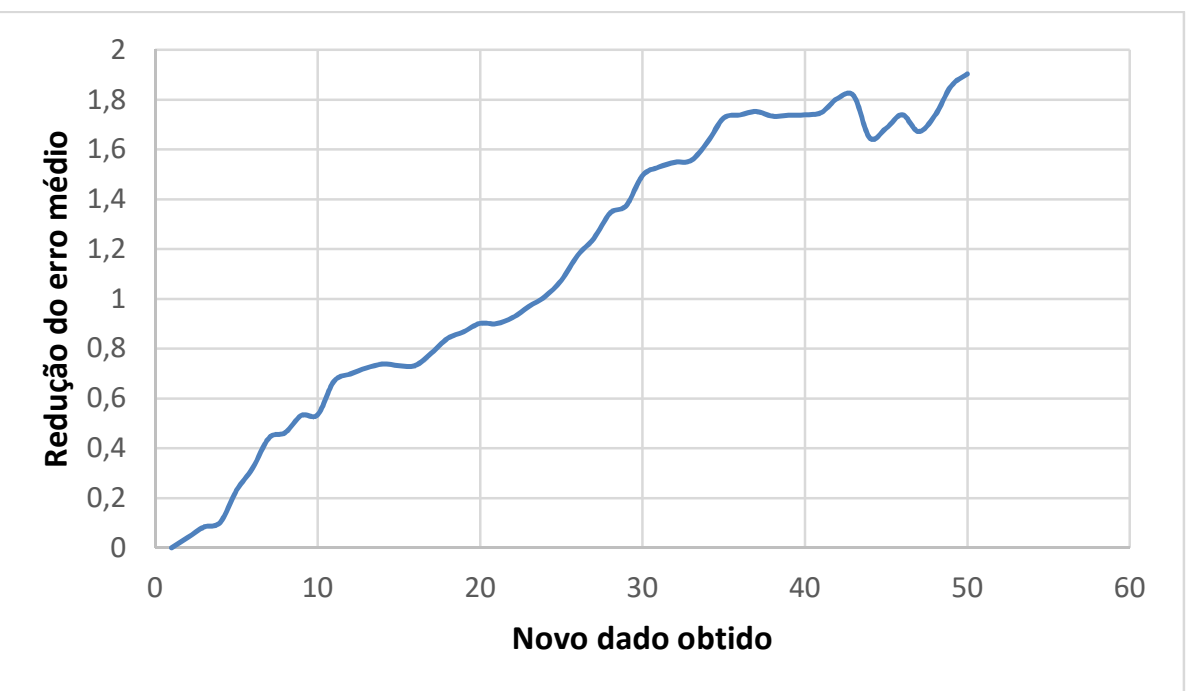

Fonte: Autor 
Em algumas baterias a segunda versão do algoritmo obteve um resultado pior, como é o caso da bateria 3, porém na média o resultado é melhor.

Uma das baterias que obtiveram uma melhora considerável foi a bateria 5 , como é possível visualizar comparando as figuras 58 e 59.

Figura 58 - Diminuição do erro médio da bateria 5 na primeira versão do algoritmo.

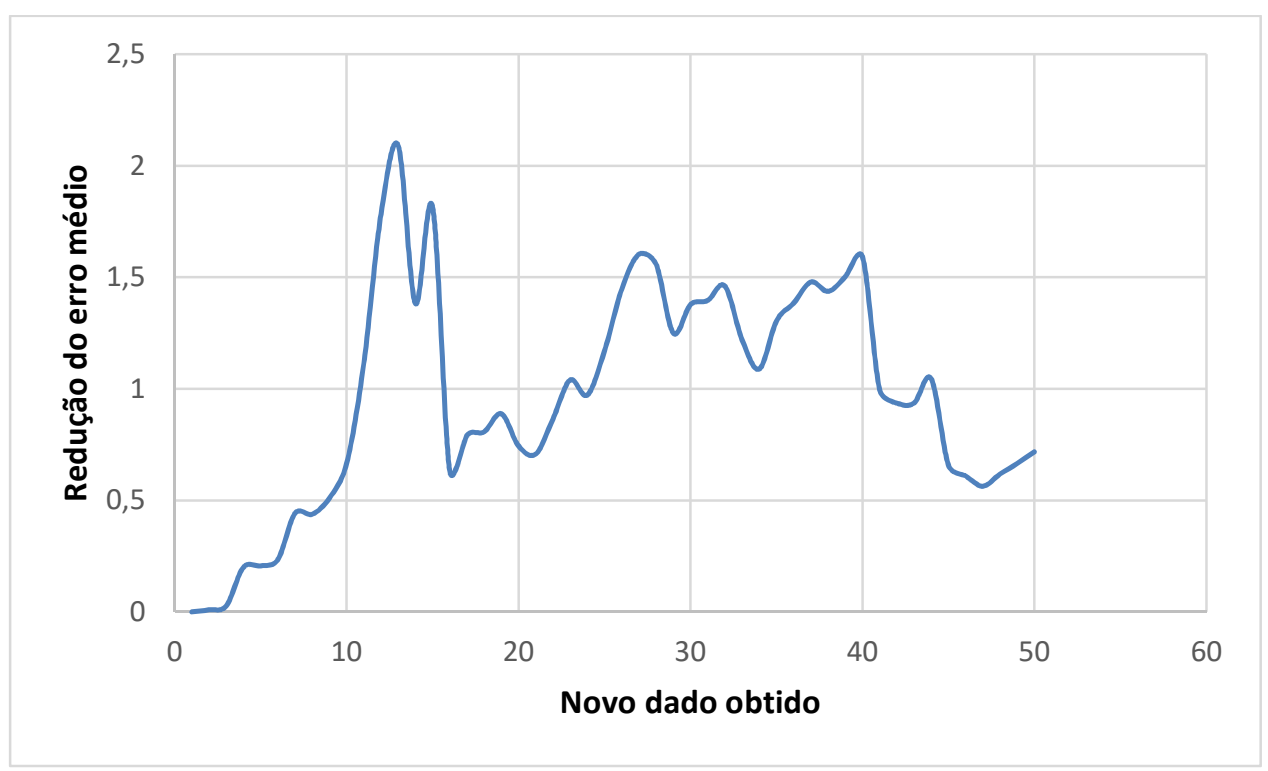

Fonte: Autor

Figura 59 - Diminuição do erro médio da bateria 5 na segunda versão do algoritmo.

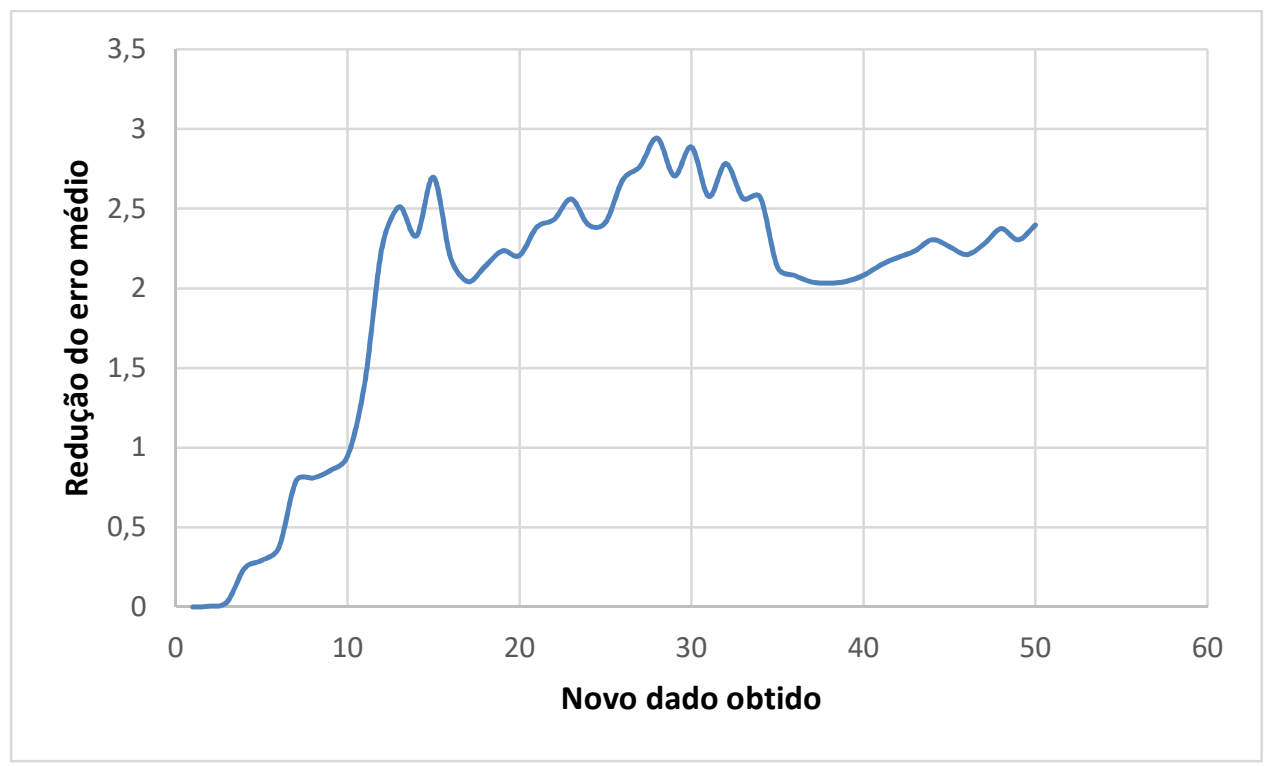

Fonte: Autor 
No exemplo da bateria 5 o objetivo da premissa foi atingido já que as quedas que ocorrem na curva na primeira versão do algoritmo ocorrem com menor intensidade na segunda versão, diminuindo os efeitos negativos de um dado que diverge do restante do conjunto de dados.

Nessa versão, é necessário calcular os resultados das duas redes neurais e algumas vezes a rede neural inicial obtém resultados melhores. Por isso, modificou-se o algoritmo uma última e terceira vez onde escolhe-se o resultado da rede neural (rede neural inicial ou rede neural adaptativa) que tem o menor erro médio no conjunto de dados já obtidos daquela bateria. Essa diferença é simples de projetar e evita que a previsão seja piorada.

Os resultados dessa versão final do algoritmo estão na tabela 12.

Tabela 12 - Erros médios da rede inicial e da rede adaptativa final.

\begin{tabular}{|c|c|c|c|}
\hline Bateria & $\begin{array}{c}\text { Erro médio para os 50 } \\
\text { dados }\end{array}$ & Erro médio adaptativo para os 50 dados & Diminuição em \% \\
\hline 1 & 6,786 & 6,383 & 5,951 \\
\hline 2 & 2,061 & 2,119 & $-2,725$ \\
\hline 3 & 6,222 & 4,318 & 30,6 \\
\hline 4 & 6,455 & 5,998 & 7,088 \\
\hline 5 & 10,646 & 8,249 & 22,519 \\
\hline 6 & 8,875 & 5,665 & 36,166 \\
\hline 7 & 8,267 & 8,269 & $-0,022$ \\
\hline 8 & 7,067 & 6,104 & 13,62 \\
\hline 9 & 9,047 & 5,749 & 36,447 \\
\hline 10 & 7,556 & 7,761 & $-2,635$ \\
\hline 11 & 2,676 & 2,808 & $-4,708$ \\
\hline 12 & 5,614 & 4,39 & 21,807 \\
\hline 13 & 6,705 & 6,845 & $-2,04$ \\
\hline 14 & 7,543 & 6,909 & 8,4 \\
\hline 15 & 8,886 & 4,429 & 50,16 \\
\hline 16 & 7,441 & 5,492 & 26,192 \\
\hline 17 & 10,243 & 10,795 & $-5,107$ \\
\hline 18 & 15,627 & 10,934 & 30,035 \\
\hline 19 & 10,269 & 10,643 & $-3,514$ \\
\hline 20 & 8,263 & 8,336 & $-0,868$ \\
\hline 21 & 9,882 & 10,013 & $-1,313$ \\
\hline Média & 7,911 & 6,772 & 14,401 \\
\hline
\end{tabular}


A tabela 12 mostra que a vantagem do algoritmo final em relação à segunda versão se dá nos conjuntos de dados onde a segunda versão do algoritmo é pior que a rede inicial. Para esses casos o erro diminuiu consideravelmente. Porém para os casos onde a segunda versão é melhor que a terceira (bateria 4 por exemplo), a diferença entre os duas é pequena.

É possível visualizar a vantagem do algoritmo adaptativo final no gráfico da bateria 10 (figura 60). Enquanto a segunda versão é melhor que a rede inicial, o algoritmo final não oferece vantagem, porém a partir da $34^{a}$ medida, ele evita que erro aumente.

Figura 60 - Comparação da diminuição do erro médio para o $2^{\circ}$ e $3^{\circ}$ algoritmo com a bateria 10.

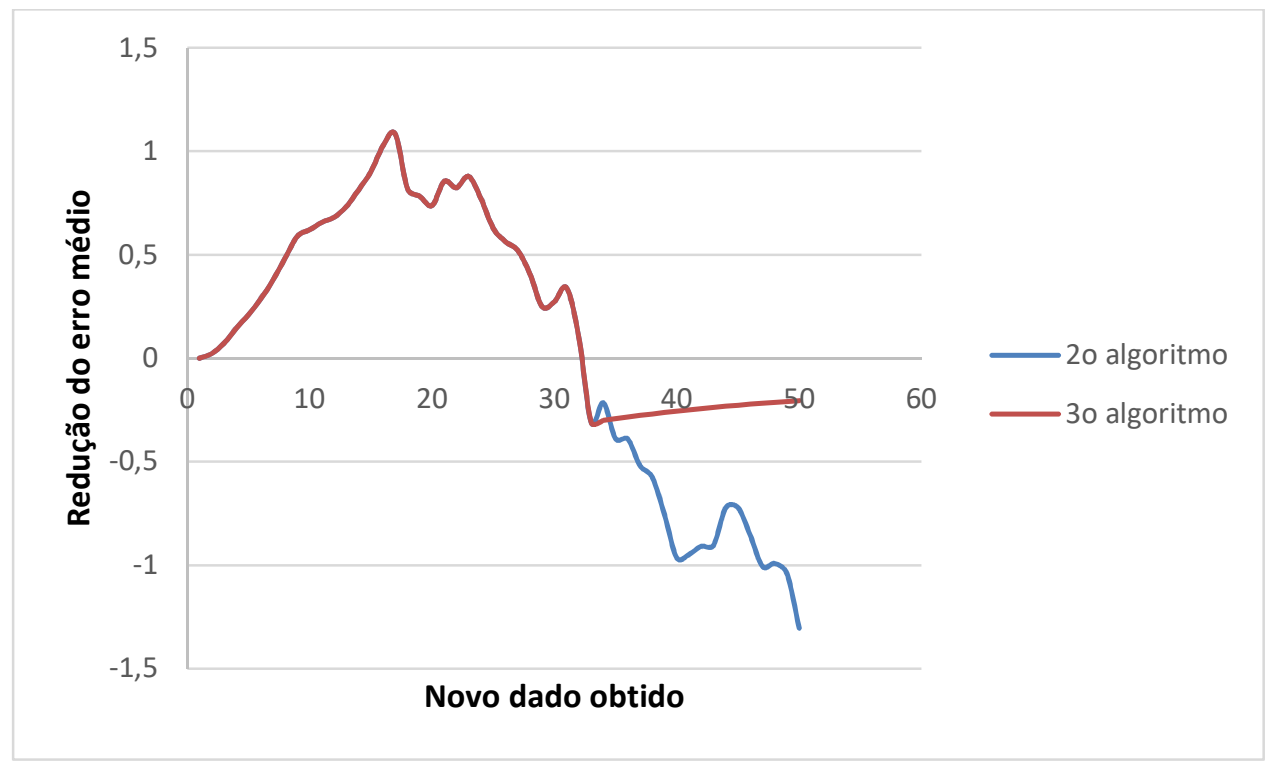

Fonte: Autor

Em suma, o algoritmo adaptativo foi atualizado para reduzir o erro médio da previsão do próximo dado a ser coletado. A diminuição do erro médio obtida pelo algoritmo adaptativo final para os 21 conjuntos de dados foi de 14,4\%. Esse algoritmo pode ser inserido no código do microcontrolador que controla a bateria.

\subsection{Melhoramento das previsões da rede neurais}

Criou-se uma segunda rede neural para prever somente o EOL das baterias. Para essa rede neural, somente uma camada interna com 25 neurônios foi usada (figura 61). A figura 61 foi criada no software MBP (LOPES; RIBEIRO, 2011). 
Figura 61 - Topologia da segunda rede neural para previsão do EOL.

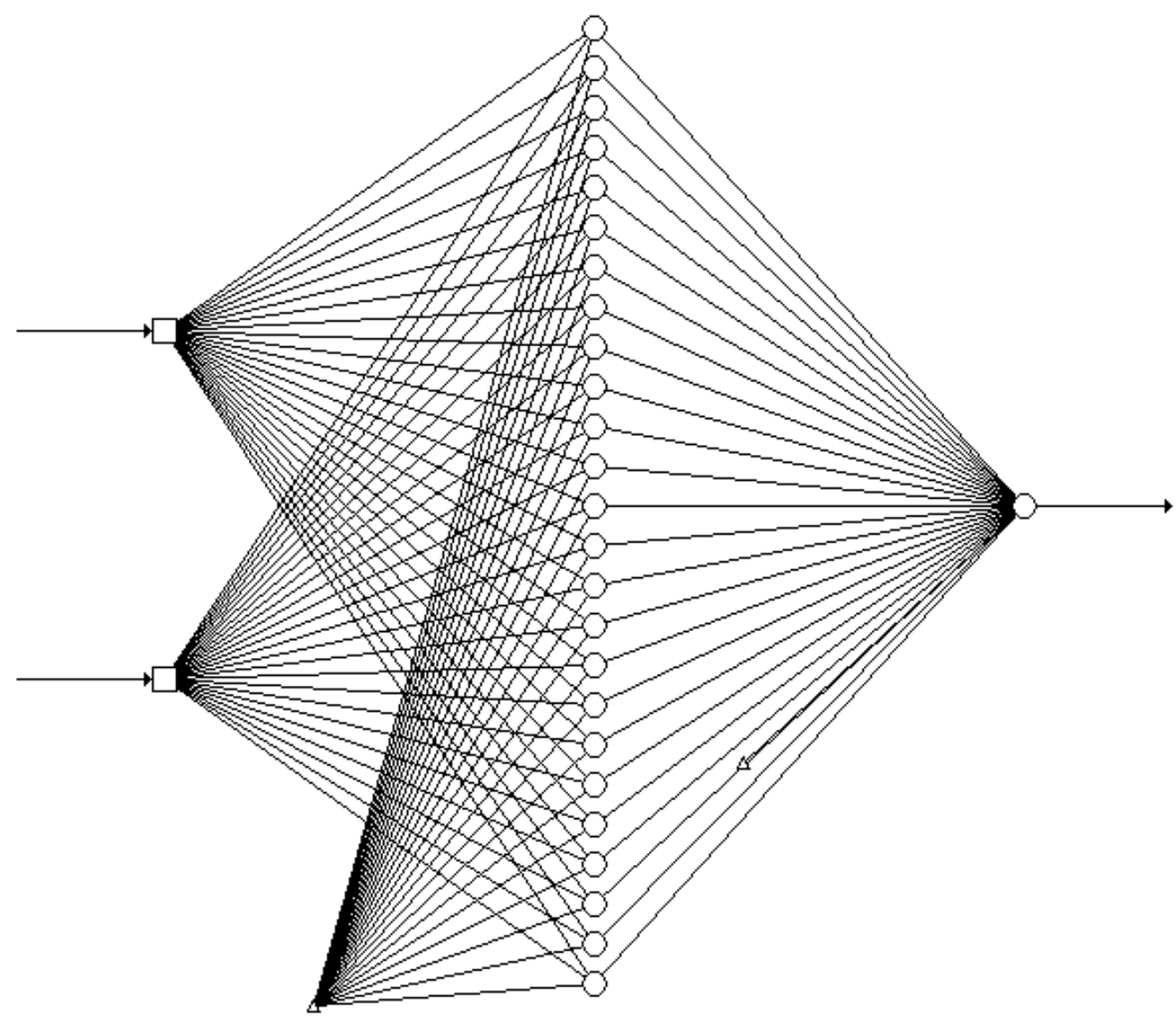

Fonte: Autor.

Os pesos obtidos para essa rede neural podem ser vistos nos apêndices.

Como era de se esperar, o maior número de erros na previsão de EOL se encontra nas baterias que possuem um $\mathrm{SOH}$ próximo do valor de definição do EOL. Os resultados dessa rede são apresentados na tabela 13. 
Tabela 13 - Resultados da segunda rede neural.

\begin{tabular}{|c|c|c|c|}
\hline & & & \\
\hline Intervalo & Número de medidas & Erro EOL & Porcentagem \\
& & & \\
\hline $20-30$ & 17 & 4 & 23,53 \\
\hline $30-40$ & 36 & 5 & 13,89 \\
\hline $40-50$ & 56 & 4 & 7,14 \\
\hline $50-60$ & 60 & 2 & 3,33 \\
\hline $60-70$ & 100 & 19 & 19 \\
\hline $70-80$ & 190 & 53 & 27,894 \\
\hline $80-90$ & 317 & 27 & 8,52 \\
\hline$>90$ & 274 & 1 & 0,36 \\
\hline Total & 1050 & 115 & 10,95 \\
\hline
\end{tabular}

Fonte: Autor

Além disso, o número de erros de EOL foi menor que o número previsto pela primeira rede neural. Isso também era esperado.

Finalmente, separou-se 80 casos que eram discrepantes para poder criar a terceira rede neural. Essa terceira rede neural possui duas camadas internas, uma com 10 neurônios e outra com 2 neurônios (figura 62). A figura 62 foi criada no software MBP (LOPES; RIBEIRO, 2011).

Figura 62 - Topologia da terceira rede neural.

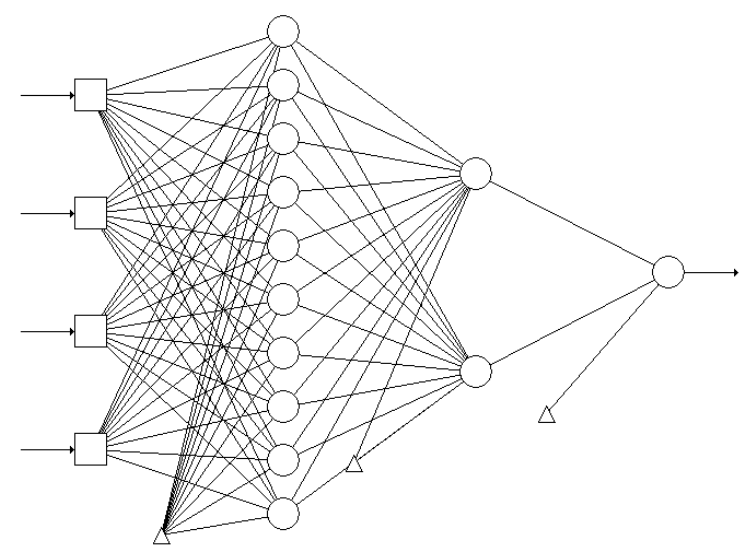

Fonte: Autor

Os pesos obtidos para essa rede neural estão nos apêndices. 
Quando a terceira rede neural indicava que o erro da previsão do SOH era maior que $8 \%$, corrigiu-se o erro. Como a terceira rede neural não indica o quanto o SOH está incorreto, somente que ele está incorreto mais ou menos que $8 \%$, foi preciso definir um fator de correção arbitrário. Esse valor foi determinado para obter o menor erro médio nesses 80 dados e um menor número de erro de EOL. Quando a rede indicava que o erro de $\mathrm{SOH}$ era $8 \%$ maior que o real, diminuiu-se o $\mathrm{SOH}$ em 9\%. Quando a rede indicava que o erro de $\mathrm{SOH}$ era $8 \%$ menor que o real, aumentou-se o $\mathrm{SOH}$ em $16 \%$. Os resultados desses 80 dados estão na tabela 14 .

Tabela 14 - Comparando os resultados da primeira e terceira redes neurais.

\begin{tabular}{|c|c|c|}
\hline & Resultados para a 1a rede neural & Resultados após a 3a rede neural \\
\hline Média do SOH dos 80 dados & 10,90 & 8,21 \\
\hline Desvio padrão dos 80 dados & 8,69 & 7.88 \\
\hline Número de erro de EOL & 53 & 34 \\
\hline
\end{tabular}

Fonte: Autor

É possível observar uma melhora na previsão do SOH e do EOL.

\subsection{Método linear}

Criou-se um programa parecido ao programa com lógica fuzzy para definir 3 equações que relacionassem a resistência e a capacitância ao $\mathrm{SOH}$. O código desse software está no apêndice $\mathrm{E}$. Ou seja, definiu-se os coeficientes $a, b, d, e, x, y$ e $z$ que obtivessem o menor erro médio para as seguintes equações:

$$
\begin{aligned}
& S O H=a * R+b \\
& S O H=d * C+e \\
& S O H=x * R+y * C+z
\end{aligned}
$$

Os mesmos dados foram para comparar os outros métodos com esse método mais simples de ser obtido.

\subsubsection{Resultados considerando somente a resistência}

Nesse modelo obteve-se a equação de reta entre a resistência e o SOH com o menor erro médio. Os valores dos coeficientes são: $a=-24.175312$ e $b=101.316772$

Os resultados são apresentados na tabela 15. 
Tabela 15 - Resultados do $1^{\circ}$ modelo linear.

\begin{tabular}{|c|c|}
\hline Erro médio do treino n 0 & 8,456 \\
\hline Erro médio do treino n 1 & 8,255 \\
\hline Erro médio do treino n 2 & 7,799 \\
\hline Erro médio do treino n 3 & 7,696 \\
\hline Erro médio do treino n 4 & 7,89 \\
\hline Erro médio do treino n 5 & 7,18 \\
\hline Erro médio do treino n 6 & 7,822 \\
\hline Erro médio do treino n 7 & 8,097 \\
\hline Erro médio do treino n 8 & 7,517 \\
\hline Erro médio do treino n 9 & 7,615 \\
\hline Erro médio de todos os dados & 7,812 \\
\hline
\end{tabular}

Como nos modelos anteriores, o erro foi maior para $\mathrm{SOH}$ menores (figura 63):

Figura 63 - Gráfico do erro médio no $1^{\circ}$ modelo linear por faixa de valor.

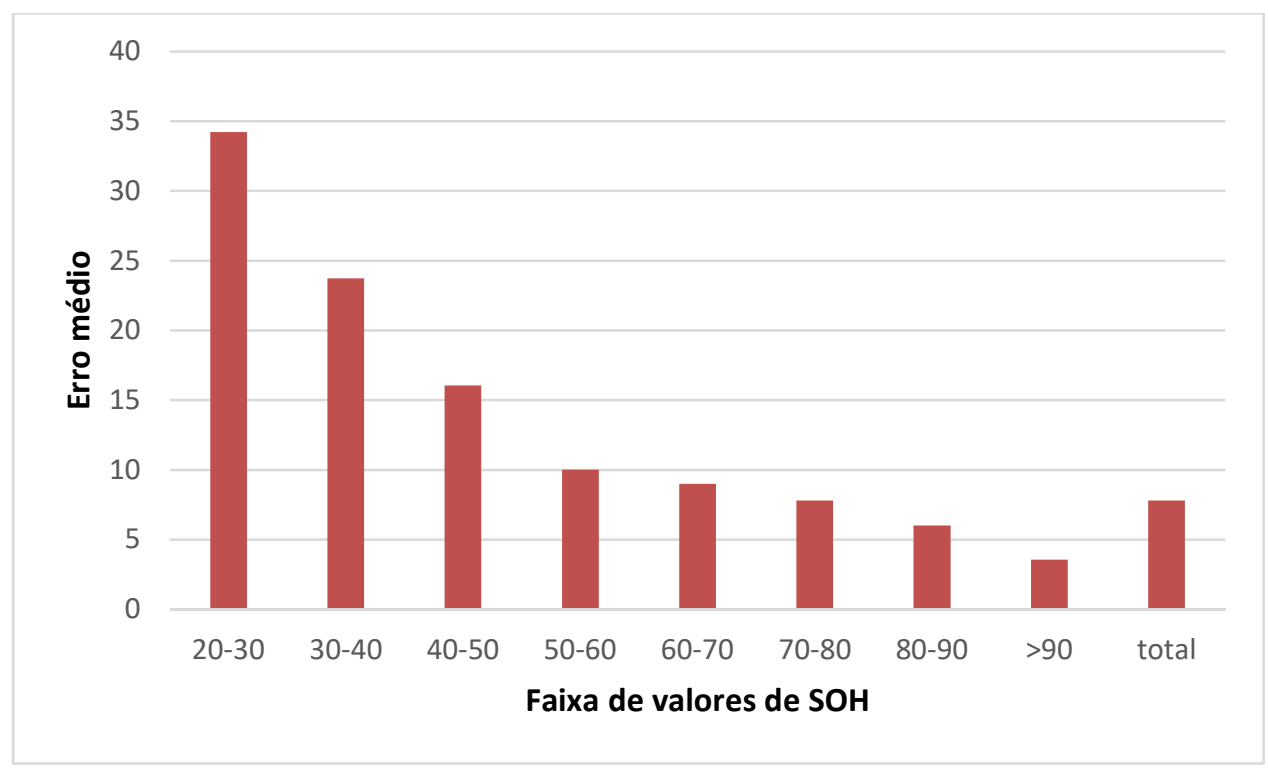

Fonte: Autor

A proporção de erros de EOL está na tabela 16. 
Tabela 16 - Resultados do $1^{\circ}$ modelo linear.

\begin{tabular}{|c|c|c|c|}
\hline Intervalo & $\begin{array}{c}\text { Número de } \\
\text { medidas }\end{array}$ & Erro médio treino & $\begin{array}{c}\text { Número de erro } \\
\text { EOL }\end{array}$ \\
\hline $20-30$ & 17 & 34,238 & 3 \\
\hline $30-40$ & 36 & 23,727 & 2 \\
\hline $40-50$ & 56 & 16,057 & 1 \\
\hline $50-60$ & 60 & 10,010 & 6 \\
\hline $60-70$ & 100 & 9,009 & 16 \\
\hline $70-80$ & 190 & 7,813 & 62 \\
\hline $80-90$ & 317 & 6,019 & 63 \\
\hline$>90$ & 274 & 3,553 & 155 \\
\hline Total & 1050 & 7,812 & \\
\hline & & & \\
\hline
\end{tabular}

\subsubsection{Resultados considerando somente a capacitância}

Nesse modelo obteve-se a equação de reta entre a capacitância e o SOH com o menor erro médio. Os valores dos coeficientes são: $d=1139.419189$ e e $=67.582153$

Os resultados estão na tabela 17.

Tabela 17 - Resultados do $2^{\circ}$ modelo linear.

\begin{tabular}{|c|c|}
\hline Erro médio do treino $\mathrm{n}$ 0 & 11,999 \\
\hline Erro médio do treino $\mathrm{n} 1$ & 12,489 \\
\hline Erro médio do treino $\mathrm{n} 2$ & 10,641 \\
\hline Erro médio do treino $\mathrm{n} 3$ & 12,652 \\
\hline Erro médio do treino $\mathrm{n} 4$ & 12,435 \\
\hline Erro médio do treino $\mathrm{n} 5$ & 11,39 \\
\hline Erro médio do treino $\mathrm{n} 6$ & 12,409 \\
\hline Erro médio do treino $\mathrm{n} 7$ & 11,705 \\
\hline Erro médio do treino $\mathrm{n} 8$ & 12,997 \\
\hline Erro médio do treino $\mathrm{n}$ 9 & 12,861 \\
\hline Erro médio de todos os dados & 12,157 \\
\hline
\end{tabular}

Fonte: Autor

Como em todos os modelos, o erro foi maior para $\mathrm{SOH}$ menores: 
Figura 64 - Gráfico do erro médio no $2^{\circ}$ modelo linear por faixa de valor.

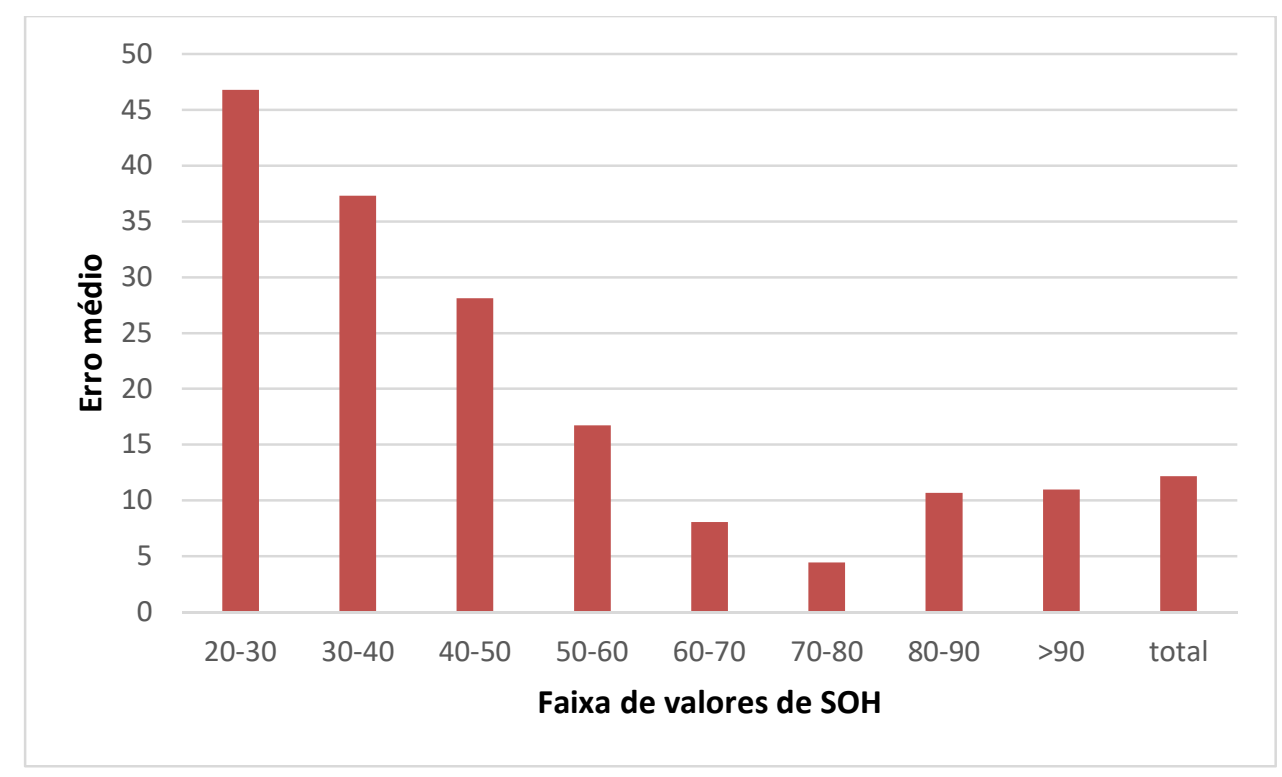

Fonte: Autor

A proporção de erros de EOL pode ser vista na tabela 18.

Tabela 18 - Resultados do $2^{\circ}$ modelo linear.

\begin{tabular}{|c|c|c|c|}
\hline Intervalo & $\begin{array}{c}\text { Número de } \\
\text { medidas }\end{array}$ & Erro médio treino & $\begin{array}{c}\text { Número de erro } \\
\text { EOL }\end{array}$ \\
\hline $20-30$ & 17 & 46,811 & 8 \\
\hline $30-40$ & 36 & 37,332 & 16 \\
\hline $40-50$ & 56 & 28,134 & 15 \\
\hline $50-60$ & 60 & 16,715 & 24 \\
\hline $60-70$ & 100 & 8,080 & 40 \\
\hline $70-80$ & 190 & 4,440 & 84 \\
\hline $80-90$ & 317 & 10,684 & 74 \\
\hline$>90$ & 274 & 10,979 & 12 \\
\hline Total & 1050 & 12,157 & 273 \\
\hline
\end{tabular}

Fonte: Autor

\subsubsection{Resultados considerando a resistência e a capacitância}

Nesse modelo obteve-se a equação do plano entre a resistência e a capacitância e o SOH com o menor erro médio. Os valores dos coeficientes são: $x=-26.324219, y=-$ 283.652344 e z=105.864311. Os resultados estão na tabela 19. 
Tabela 19 - Resultados do $3^{\circ}$ modelo linear.

\begin{tabular}{|c|c|}
\hline Erro médio do treino $\mathrm{n}$ 0 & 8,169 \\
\hline Erro médio do treino $\mathrm{n}$ 1 & 8,011 \\
\hline Erro médio do treino $\mathrm{n}$ 2 & 7,758 \\
\hline Erro médio do treino $\mathrm{n} 3$ & 7,491 \\
\hline Erro médio do treino $\mathrm{n}$ 4 & 7,514 \\
\hline Erro médio do treino $\mathrm{n} 5$ & 7,056 \\
\hline Erro médio do treino $\mathrm{n}$ 6 & 7,608 \\
\hline Erro médio do treino $\mathrm{n} 7$ & 8,031 \\
\hline Erro médio do treino $\mathrm{n}$ 8 & 7,297 \\
\hline Erro médio do treino $\mathrm{n}$ 9 & 7,545 \\
\hline Erro médio de todos os dados & 7,625 \\
\hline
\end{tabular}

Como em todos os modelos, o erro foi maior para SOH menores (figura 65).

Figura 65 - Gráfico do erro médio no $3^{\circ}$ modelo linear por faixa de valor.

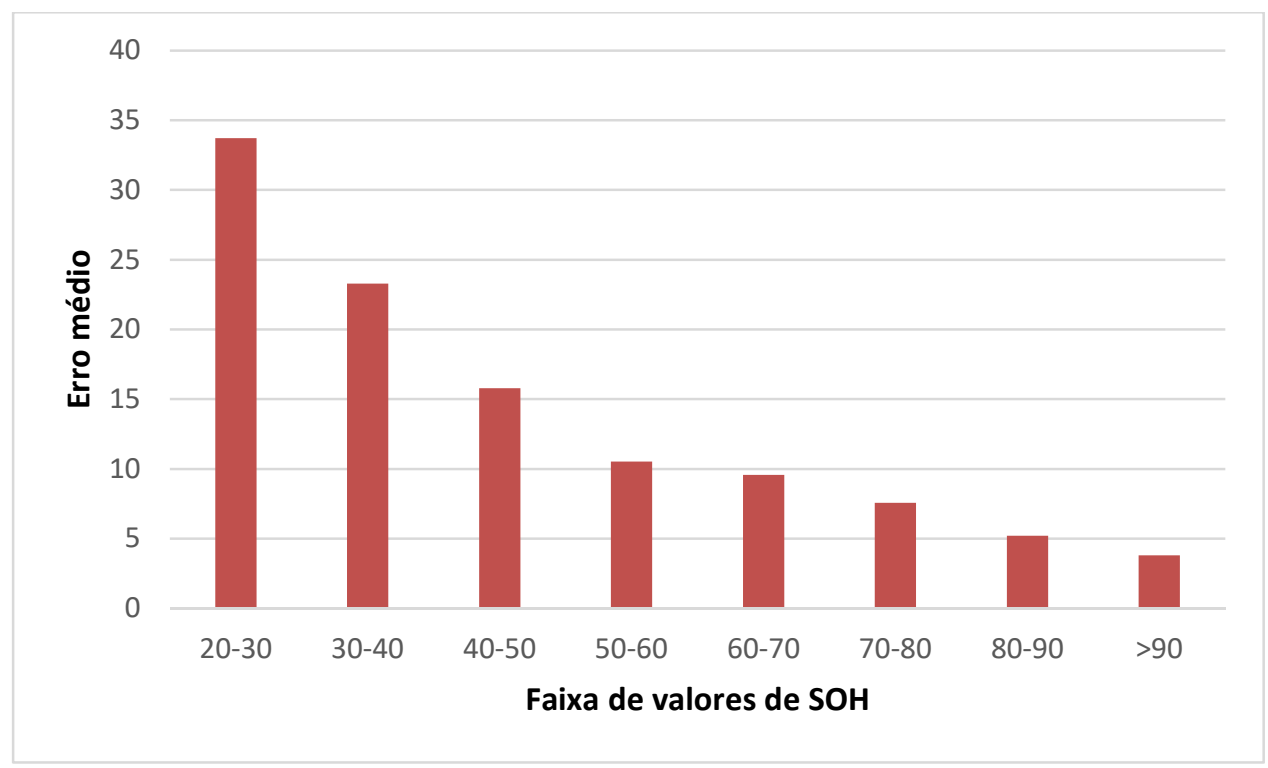

Fonte: Autor

Esse é o melhor modelo linear. A proporção de erros de EOL está na tabela 20. 
Tabela 20 - Resultados do $3^{\circ}$ modelo linear.

\begin{tabular}{|c|c|c|c|}
\hline Intervalo & $\begin{array}{c}\text { Número de } \\
\text { medidas }\end{array}$ & $\begin{array}{c}\text { Erro médio } \\
\text { treino }\end{array}$ & $\begin{array}{c}\text { Número de erro } \\
\text { EOL }\end{array}$ \\
\hline $20-30$ & 17 & 33,703 & 3 \\
\hline $30-40$ & 36 & 23,280 & 2 \\
\hline $40-50$ & 56 & 15,7696 & 2 \\
\hline $50-60$ & 60 & 10,510 & 7 \\
\hline $60-70$ & 100 & 9,575 & 19 \\
\hline $70-80$ & 190 & 7,567 & 62 \\
\hline $80-90$ & 317 & 5,193 & 53 \\
\hline$>90$ & 274 & 3,794 & 2 \\
\hline Total & 1050 & 7,625 & 150 \\
\hline
\end{tabular}

Fonte: Autor

Esse é método mais simples computacionalmente tanto para treinamento quanto para implementação e serve de parâmetro de comparação. 


\section{Análise dos resultados}

A tabela 21 compara os resultados da rede neural, lógica fuzzy e do método linear:

Tabela 21 - Comparação dos resultados.

\begin{tabular}{|c|c|c|c|}
\hline Método & Erro médio & Número de erro de EOL & Erro de EOL em \% \\
\hline Rede neural & 6,958 & 141 & 13,43 \\
\hline Fuzzy ordem 0 & 7,053 & 147 & 14 \\
\hline Fuzzy ordem 1 & 6,818 & 150 & 14,29 \\
\hline Linear - resistência & 7,812 & 155 & 14,76 \\
\hline Linear - capacitância & 12,157 & 273 & 26 \\
\hline $\begin{array}{c}\text { Linear - resistência e } \\
\text { capacitância }\end{array}$ & 7,625 & 150 & 14,29 \\
\hline
\end{tabular}

Fonte: Autor

O método que apresentou os melhores resultados foi a rede neural já que obteve o segundo menor erro médio e o menor número de erros de previsão de EOL. O método utilizando lógica fuzzy ofereceu resultados melhores que os métodos lineares, o que justifica o seu uso já que é um método de implementação mais simples que a rede neural, pois o treinamento do sistema fuzzy é mais simples de ser feita e não necessita de um grande conjunto de dados. Adicionalmente, o método de lógica fuzzy de ordem 1 obteve o menor erro médio.

Quanto maior a complexidade do método, menor o erro médio. Isso é possível de ser visto já que o método usando lógica fuzzy de ordem 0 tem o maior erro seguido da lógica fuzzy de ordem 1 e finalmente a rede neural. Para analisar a complexidade do método deve se levar em conta tanto a complexidade do treinamento, a quantidade necessária de dados prévios já que isso envolve testes extensos e a complexidade da implementação do método no sistema embarcado que monitora a bateria estacionária.

Quando usados em microcontroladores atuais, essas técnicas não usam muito espaço de memória. A rede neural estudada, por exemplo, tem 83 parâmetros. Nesse aspecto, o método de Sugeno de ordem 1 da lógica fuzzy é o mais ineficiente já que usa 147 parâmetros. Isso poderia ser evitado diminuindo o número de funções de pertencimento, utilizando 3 ou 5 categorias em vez de 7. Isso diminuiria a complexidade do método e provavelmente não influenciaria tanto o resultado já que o método de Sugeno de ordem 0 teve um resultado parecido. Além disso, alguns parâmetros não são utilizados já que não há dados que possuem a característica definida e poderiam ser eliminados. Por exemplo, não há dado de bateria com 
resistência na categoria "muito alta" e capacitância na categoria "muito alta", ou seja, não há necessidade de definir um parâmetro para um caso como esse.

Além disso, é possível perceber que um erro médio menor na previsão do SOH não necessariamente significa um número menor de erro de previsão de EOL. Como o objetivo era minimizar o erro médio, os dados que estão longe da média apresentam erros maiores. Isso pode ser visualizado nos gráficos que separam o erro médio pela faixa de valor de $\mathrm{SOH}$. Como foi obtido um maior número de medidas que não tinham atingido o EOL, esses valores possuem erro médio menor. O número de erro de definição de EOL é alto para todos os métodos pois há um número alto de dados que estão próximos do valor de definição. Isso pode ser contornada em soluções de baterias estacionárias com ênfase em aplicações de segurança já que essa aplicação permite diversas medições em um longo período de tempo. Portanto o registro da evolução dessa medição seria útil para corrigir discrepâncias relacionadas aos métodos.

O método de redução de erro de EOL é útil para reduzir essa distorção e é o método mais efetivo para obter o SOF da bateria. Esse seria o melhor método para avisar quando a bateria precisa ser trocada e pode ser usado em aplicações ligadas à segurança onde a bateria não é usada a maioria do tempo (a bateria do desfibrilador por exemplo). Esse método pode ser útil também em aplicações onde não é possível realizar muitos testes com a bateria. Outra utilidade desse método é otimizar a escolha do valor de EOL que é uma escolha crítica para aplicações de segurança.

Criou-se outro método para diminuir os erros da rede neural, o método adaptativo, que foca em diminuir o erro médio do conjunto de teste. Esse método tem objetivos diferentes e deve ser usado para casos diferentes. O método adaptativo só pode ser usado em casos que é possível calcular a capacidade da bateria de outra forma ou inferir ela de alguma forma. Esse método pode ser útil em baterias estacionárias que podem ser descarregadas de tempo em tempo, calculando assim a real capacidade da bateria. Para essas baterias o valor correto do SOH pode ser mais útil que o SOF.

Uma desvantagem presente nos métodos baseados em redes neurais é a necessidade de um conjunto de teste treinado e isso demanda tempo e custo. Uma forma de minimizar essa desvantagem é estabelecer uma comunicação com o microcontrolador para atualizar os parâmetros toda vez que novos dados são obtidos. 
Para o método adaptativo isso pode ser benéfico pois o número de dados obtidos aumenta consideravelmente.

Outra desvantagem que a análise não levou em conta é a influência da temperatura e da corrente de descarga na estimativa do SOH. Essas variáveis influenciam e podem restringir o uso desses métodos em certas aplicações. Para medir a influência dessas variáveis seria necessário obter um número maior de dados de testes e aumentar a complexidade do circuito de medição. Essas variáveis podem ser analisadas em trabalhos futuros como também o embarque do sistema em uma aplicação específica. O método usando lógica fuzzy pode ser melhor em situações que possuem alta variedade de temperatura já que esse método não é tão dependente da base de dados e incluir mais uma variável ao sistema aumentaria o custo e a dificuldade na obtenção de dados.

No final, apresentou-se diversos métodos, cada um com suas vantagens. A vantagem dessa diversidade de métodos que usam inteligência artificial é a possibilidade de adaptar cada método a sua aplicação. Quanto mais complexo o método, melhor são os resultados, porém todos são possíveis de implementar usando um microcontrolador em um sistema embarcado. 


\section{Conclusão}

Em suma, o objetivo foi atingindo já que os diversos métodos apresentados estimam a capacidade atual de uma bateria estacionária com dados coletados em um teste feito por um microcontrolador.

Como foi visto, novas soluções para monitoramento de baterias de íons de lítio são intensivamente estudadas já que esse tipo de bateria é cada vez mais útil para diversas aplicações. O mercado para esse tipo de bateria está crescendo a cada ano graças às diversas vantagens que esse tipo de bateria apresenta. Isso também justifica a busca por novas formas de gerenciar e monitorar essas baterias.

Nesse trabalho foram mostradas as diversas vantagens das baterias de íon de lítio e as suas características. Além disso as diferentes causas do envelhecimento dessas baterias foram descritas.

A teoria dos métodos de rede neural, lógica fuzzy e adaptatividade foi estudada e diversos métodos usando essas teorias foram apresentados e os resultados analisados. Esses métodos oferecem soluções simples e diferenciadas para a medição do $\mathrm{SOH}$ de baterias de íon de lítio.

Portanto a escolha do método a ser utilizado depende da aplicação e das limitações do projeto e do microcontrolador. Apesar de todos os métodos serem simples, alguns dependem de maior tratamento dos dados coletados (como por exemplo, a rede neural) e outros dependem de condições específicas da aplicação (como por exemplo, o método adaptativo). 


\section{REFERÊNCIAS}

AMARI, S.; CICHOCKI, A. Adaptive Blind Signal Processing - Neural Network Approaches, PROCEEDINGS OF THE IEEE, vol. 86, n. 10, 1998.

APPLE. Disponível em: https://support.apple.com/pt-br/HT208387. Acesso em: 04 abr. 2019.

ARMAND, M.; TARASCON, J.-M. Building better batteries, Nature, n. 451, 2008, p $652-657$.

ARORA, P.; WHITE, R.; DOYLE, M Capacity fade mechanisms and side reactions in lithium-ion batteries, Journal of the Electrochemical Society, n. 145.10, 1998, p 36473667.

BAI, G. et al, A generic model-free approach for lithium-ion battery health management, Applied Energy, n. 135, 2014, p247-260.

BRODD, R. J. Comments on the History of Lithium-Ion Batteries. Broddarp of Nevada, 2001. Disponível em: https://www.electrochem.org/dl/ma/201/pdfs/0259.pdf Acesso em: 02 dez. 2020.

BUCHMANN, I. Battery University- Disponível em: http://batteryuniversity.com/learn/. Acesso em: 30 de jul. 2015.

CANOVAS, S.R.M.; CUGNASCA, C.E. Um Mapeamento de Modelos Adaptativos para Dispositivos Adaptativos Guiados por Regras, X Workshop de Tecnologia Adaptativa, São Paulo, 2016.

CHAN C.C.; LO E.W.C.; SHEN W. The available capacity computation model based on artificial neural network for lead-acid batteries in electric vehicles, Journal of Power Sources, n. 87, 2000, p 200-204.

CHEN, C.; PECHT, M. Prognostics of lithium-ion batteries using model-based and data-driven methods, Proceedings of the IEEE 2012 Prognostics and System Health Management Conference, Beijing, 2012, p. 1-6.

CHEN, Z. et al. Online battery state of health estimation based on Genetic Algorithm for electric and hybrid vehicle applications, Journal of Power Sources, n. 240, 2013, p 184-192. 
CHIANG, Y-H.; SEAN, W-Y.; KE J-C. Online estimation of internal resistance and open-circuit voltage of lithium-ion batteries in electric vehicles, Journal of Power Sources, n. 196, 2011, p 3921-3932.

CHOI, J. W.; AURBACH, D. Promise and Reality of Post-Lithium-lon Batteries with High Energy Densities, Nature Reviews Materials, March 2016.

CICHOCKI, A. et al. Self-Adaptive Neural Networks For Blind Separation Of Sources, 1996 IEEE International Symposium on Circuits and Systems. Circuits and Systems Connecting the World. ISCAS 96, Atlanta, GA, USA, 1996, p. 157-160 vol.2.

COLEMAN, M.; HURLEY, W.G; LEE, C.K. An improved battery characterization method using a two-pulse load test, IEEE Transactions on Energy Conversion, 2008, vol. 23, no. 2, p. 708-713.

DUBARRY, M. et al. State of health battery estimator enabling degradation diagnosis Model and algorithm description, Journal of Power Sources, n. 360, 2017, p 59-69.

FLEISCHER, C. et al, Self-learning state-of-available-power prediction for lithium-ion batteries in electrical vehicles, IEEE Vehicle Power and Propulsion Conference, Seoul, 2012, pp. 370-375.

GOLD PEAK INDUSTRIES LTD, Lithium lon technical handbook, 2003. Disponível em http://large.stanford.edu/courses/2015/ph240/uang2/docs/li-handbook.pdf. Acessado em 02 dez de 2020.

GROOT, J. State-of-Health Estimation of Li-ion Batteries: Cycle Life Test Methods, Chalmers University Of Technology, 2012. Dissertação - CHALMERS UNIVERSITY OF TECHNOLOGY Göteborg, Sweden 2012

HE, W. et al. Prognostics of lithium-ion batteries based on Dempster-Shafer theory and the Bayesian Monte Carlo method, Journal of Power Sources, n. 196, 2011, p 10314- 10321.

HU, C. et al, Online estimation of lithium-ion battery capacity using sparse Bayesian learning, Journal of Power Sources, n. 289, 2015, p 105-113.

IDOTA, Y. et al. Tin-Based Amorphous Oxide: A High-Capacity Lithium-Ion-Storage Material, Science, n. 276, 1997, p 1395-1397. 
JUANG, L. W. Online battery monitoring for state-of-charge and power capability prediction, 2010, 157p. Dissertação (Mestrado) - University of Wisconsin - Madison 2010.

KOHAVI, R. A Study of Cross-Validation and Bootstrap for Accuracy Estimation and Model Selection, International Joint Conference on Articial Intelligence (IJCAI), 1995.

LAKKIS, M.E. et al, Combined battery SOC/SOH estimation using a nonlinear adaptive observer, 14th annual European Control Conference, Linz, Austria, 2015.

LE, D.; TANG, X. Lithium-ion Battery State of Health Estimation Using Ah-V Characterization, Annual conference of the prognostics and health management society, 2011 , vol. 73 , no. 3, p. 367-373..

LIN, C.; LEE, C.S., Neural Network Based Fuzzy Logic Control And Decision System, IEEE TRANSACTIONS ON COMPUTERS, 1991, vol. 40, n. 12, p. 1320-1336.

LIN, C.J.; XU, Y.L. A Self-Adaptive Neural Fuzzy Network With Group-Based Symbiotic Evolution And Its Prediction Applications, Fuzzy Sets and Systems, n. 157, 2016, p 1036 - 1056.

LINDEN; REDDY. Product Engineering Processes. Disponível em: http://web.mit.edu/2.009/www/resources/mediaAndArticles/batteriesPrimer.pdf. Acesso em: 28 de jul. 2015.

LIU, D. et al. Satellite lithium-ion battery remaining cycle life prediction with novel indirect health indicator extraction, Energies, n. 6, 2013, p 3654-3668.

LOPES, N.; RIBEIRO, B. An evaluation of multiple feed-forward networks on GPUs, International Journal of Neural Systems (IJNS), n. 21, 2011, p 31-47.

LU, L. et al. A review on the key issues for lithium-ion battery management in electric vehicles, Journal of Power Sources, n. 226, 2013, p 272-288.

MATSUMOTO, E.Y.; HERNANDEZ-DEL-MORAL, E. Improving regression predictions using individual point reliability estimates based on critical error scenarios, Information Sciences, n. 374, 2016, p 65-84. 
MUKHERJEE, A. Advances in battery management using neural networks and fuzzy logic, School of Electrical and Computer Engineering, Cornell University, Ithaca, 2013.

NETO, J.J. Adaptative rule-driven devices - general formulation and a case study, CIAA'2001 Sixth International Conference on Implementation and Application of Automata, Pretoria, South Africa, 2001, p 234-250.

O'GORMAN, C.C. et al. Artificial Neural Network Simulation of Battery Performance, Proceedings of the Thirty-First Hawaii International Conference on System Sciences, 1998, vol. 5, pp. 115-121.

PAPLINSKI, A.P. Neuro-Fuzzy Computing, 2005.

PASSERINI, S.; SCROSATI B. Lithium and Lithium-Ion Batteries: Challenges and Prospects. Electrochemical Society Interface. 2016 Jan, p 85-87.

REMMLINGERA, J. et al. State-of-health monitoring of lithium-ion batteries in electric vehicles by on-board internal resistance estimation, Journal of Power Sources, n. 196, 2011, p 5357-5363.

SAHA, B. et al. An integrated approach to battery health monitoring using bayesian regression and state estimation, IEEE Autotestcon 2007 Sep 17 p. 646-653.

SAHA, B.; GOEBEL, K.; CHRISTOPHERSEN, J. Comparison of Prognostic Algorithms for Estimating Remaining Useful Life of Batteries, Transactions of the Institute of Measurement and Control. 2009 Jun, p.293-308.

SALKIND, A.J. et al. Determination of state-of-charge and state-of-health of batteries by fuzzy logic methodology, Journal of Power Sources, n. 80, 1999, p 293-300.

SANTOS, J.M.N. Dispositivos Adaptativos Cooperantes, X Workshop de Tecnologia Adaptativa, São Paulo, 2016.

SBARUFATTI, C. et al. Adaptive prognosis of lithium-ion batteries based on the combination of particle filters and radial basis function neural networks, Journal of Power Sources, n. 344, 2017, p 128-140.

SCORSATI, B.; CROCE, F.; PANERO, S. Progress in lithium polymer battery R\&D, Journal of Power Sources, n. 100, 2001, p 93-100. 
SINGH, P.; REISNER, R. Fuzzy Logic-Based State-of-Health Determination of Lead Acid Batteries, 24th Annual International Telecommunications Energy Conference 2002 Oct p. 583-590.

SINGH, P. et al Design and implementation of a fuzzy logic-based state-of-charge meter for Li-ion batteries used in portable defibrillators, Journal of power sources, 2006.

SUOZZO, C. Lead-acid battery aging and state of health diagnosis, Tese (doutorado), The Ohio State University, 2008

TAIYOU RESEARCH. Global Market for Lithium-Ion Batteries - Forecast, Trends and Opportunities 2014-2020, 2013

THE ECONOMIST. In search of the perfect battery 2008- Disponível em:

http://www.economist.com/sciencetechnology/tq/PrinterFriendly.cfm?story id=10789 409. Acesso em: 28 jul. 2015.

TORREY, L.; SHAVLIK, J. (2009). Transfer learning. Handbook of Research on Machine Learning Applications, IGI Global, 2009.

VALLE, M.E. O Método de Inferência de Takagi-Sugeno para Sistemas Baseados em Regras Fuzzy, Unicamp, 2015.

WANG, Y. et al. Probability based remaining capacity estimation using data-driven and neural network model, Journal of Power Sources, n. 315, 2016, p 199-208.

WHITTLE, J. et al. RELAX: Incorporating Uncertainty into the Specification of SelfAdaptive Systems, 17th IEEE International Requirements Engineering Conference 2009 Aug 31 p. 79-88.

WU. J.; ZHANG, C.; CHEN, Z. An online method for lithium-ion battery remaining useful life estimation using importance sampling and neural networks, Applied Energy, n. 173, 2016, p 134-140.

XING, Y. et al. Battery management systems in electric and hybrid vehicles, Energies, n. 4, 2011, p 1840-1857. 
YANGA, D. et al. A neural network based state-of-health estimation of lithium-ion battery in electric vehicles, Energy Procedia, n. 105, 2017, p 2059-2064.

ZENATI, A.; DESPREZ, P.; RAZIK, H. Estimation of the SOC and the SOH of li-ion batteries, by combining impedance measurements with the fuzzy logic inference, IECON, Glendale, United States, Nov 2010, p.1767-1772. 


\section{APÊNDICES}

\section{APÊNDICE A - Funções do FW de aquisição de dados.}

\section{Função de carga}

if( BatTest == 3 ) //Bateria em carga

\{

$v=$ BatteryCharge();// definição do PWM de carga e retorna a tensão da bateria.

// Bateria é medida no primeiro ciclo do PWM, nos demais ciclos retorna FFFF

//Ou seja, retorna o valor da bateria a cada 0.1 segundos

if $(v !=0 x f f f)$

\{

$t=$ TickCount \% 300; // 1 log a cada 30 segundos (medição de ADC a cada 0.1s)

TickCount++;

vmed $=v m e d+v$;

vmedcount++;

$/ / v=(v * 1611+2048) / 4096+49$

// Registrar tensão do carregador da bateria

// Divisor de tensão 47kOhms + 12kOhms

$v 1=A D R e a d(1<<6) ; / / A D 0.6$ Charger out

if $(t==0 \& \& v m e d !=0)$

\{

$v=v m e d / v m e d c o u n t$

$v \operatorname{vmed}=0$;

vmedcount $=0$;

LogWrite $(v>>8, v \& 0 x F F, v 1>>8, v 1$ \& 0xFF, 0x01); // Registrar medição da bateria LogCount++;

$v=(v * 3291 * 48) / 4095 / 330+46 ; / /$ tensao em centivolts

if $(v>418)$ // Tensão que para de carregar

BatTest $=4$;

$v=(v+5) / 10 ; / /$ tensao em decivolts

$v=((v / 10)<4)+\quad$ // algarismo das dezenas

( $v \% 10) ; \quad$ // algarismo das unidades

Display7Seg( v, 3 ); //coloca no display a tensao

\}

\} // if $v$

\} // if BatTest 


\section{Função de descarga nos primeiros $400 \mathrm{~ms}$}

switch( BatTest)

\{

case 4:

CLR_IO( 1, 5 ); // P1.5 Load Battery - Desliga carregador da bateria $j 1++$;

if(j1>100) //Espera 2 segundos

\{

BatTest=5;

$j 1=0$;

$v=A D R e a d(1<<3) ; / / A D 0.3$ Bat

LogWrite $(v>>8, v \&$ OxFF, 0x00, 0x00, 0x03 ); // Registrar medição da bateria LogCount++;

\}

break;

case 5: // inicia a descarga e faz a primeira medição após

SET_IO( 1, 15); // Aciona o primeiro transistor de controle da descarga

SET_IO( 1, 6 ); // Aciona o segundo transistor de controle da descarga

BatTest $=6$;

$v=A D R e a d(1<<3) ; / / A D 0.3 B$ Bat

LogWrite $(v>>8, v \&$ OxFF, 0x00,0x00, 0x03 ); // Registrar medição da bateria LogCount++;

break;

case 6: //medição a cada $20 \mathrm{~ms}$ até o final

$v=$ ADRead $(1<<3) ; / / A D 0.3$ Bat

LogWrite ( $v>>$ 8, v \& 0xFF, 0x00, 0x00, 0x03 ); // Registrar medição da bateria LogCount++;

j1++;

if(j1>17)

\{

BatTest=2; //regime de descarga normal

$j 1=0$;

\}

break;

\} //fim do switch 


\section{Função de descarga}

if( BatTest == 2)// função de descarga

\{

$v=$ BatteryCheck(); // Mede tensão da bateria e controla o PWM de acordo devolve a tensão da bateria a cada 250ms ou devolve Oxffff

if $(v !=0 x f f f)$

\{

$t=$ TickCount \% 120; // 1 log a cada 30 segundos

TickCount++;

if $(t==0)$

\{

LogWrite ( $v$ >> 8, v \& 0xFF, 0x00, 0x00, 0x02 ); // Registrar medição da bateria LogCount++;

\}

$v=(v * 3291 * 48) / 4095 / 330+46 ; / /$ tensao em centivolts

if $((v<=300)$ || (LogCount $>2047))$ //tensão menor que 3V ou log cheio

\{

BatTest $=0 ; / /$ Estado final do teste

\}

$v=(v+5) / 10 ; / /$ tensao em decivolts

$v=((v / 10)<4)+/ /$ algarismo das dezenas

( $v \% 10)$; // algarismo das unidades

Display7Seg( $v, 2)$;

\}//v! =0xfff;

\}$/ /$ if BatTest $==2$ 


\section{APÊNDICE B - Extratos do SW criado para análise com rede neural}

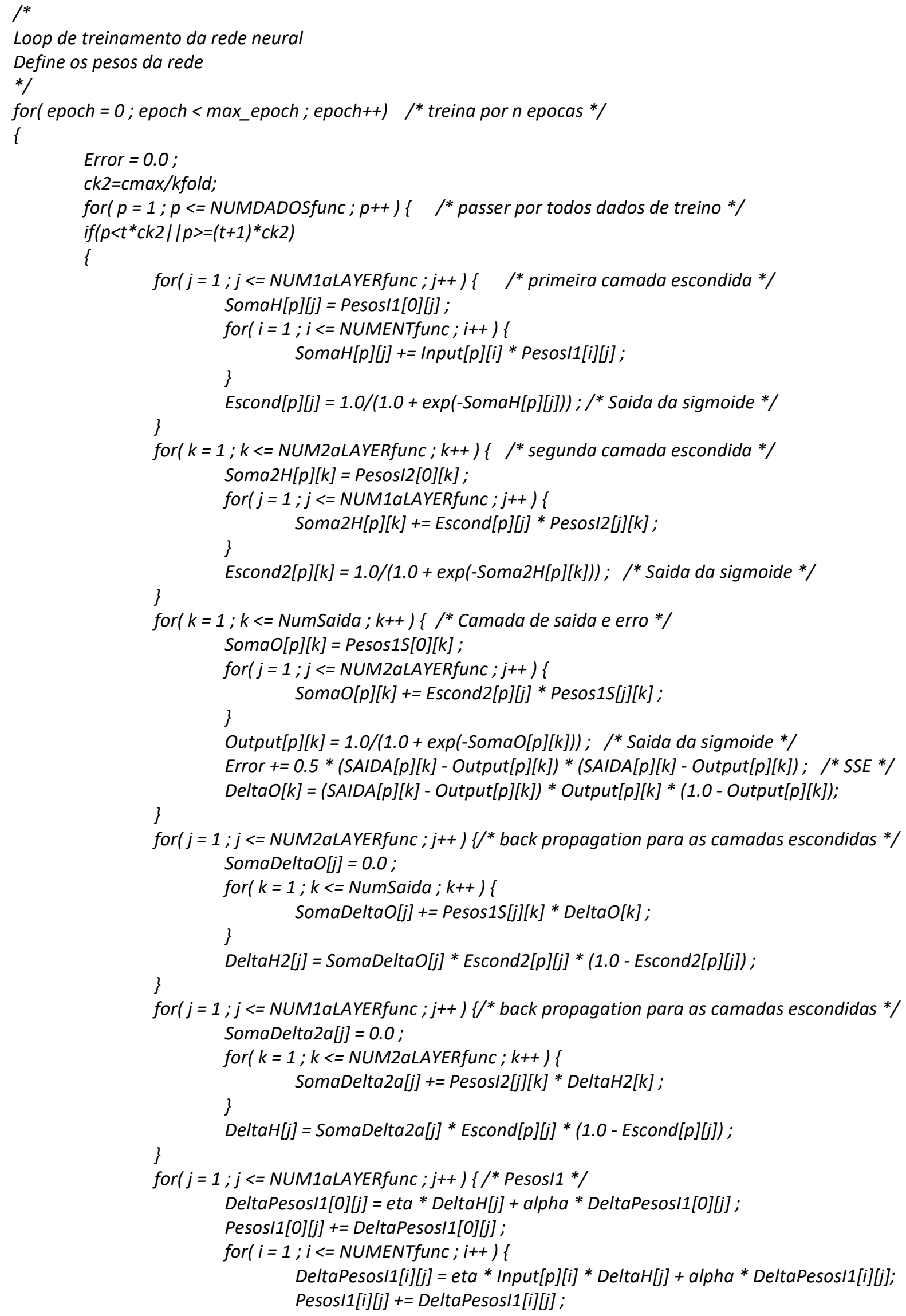




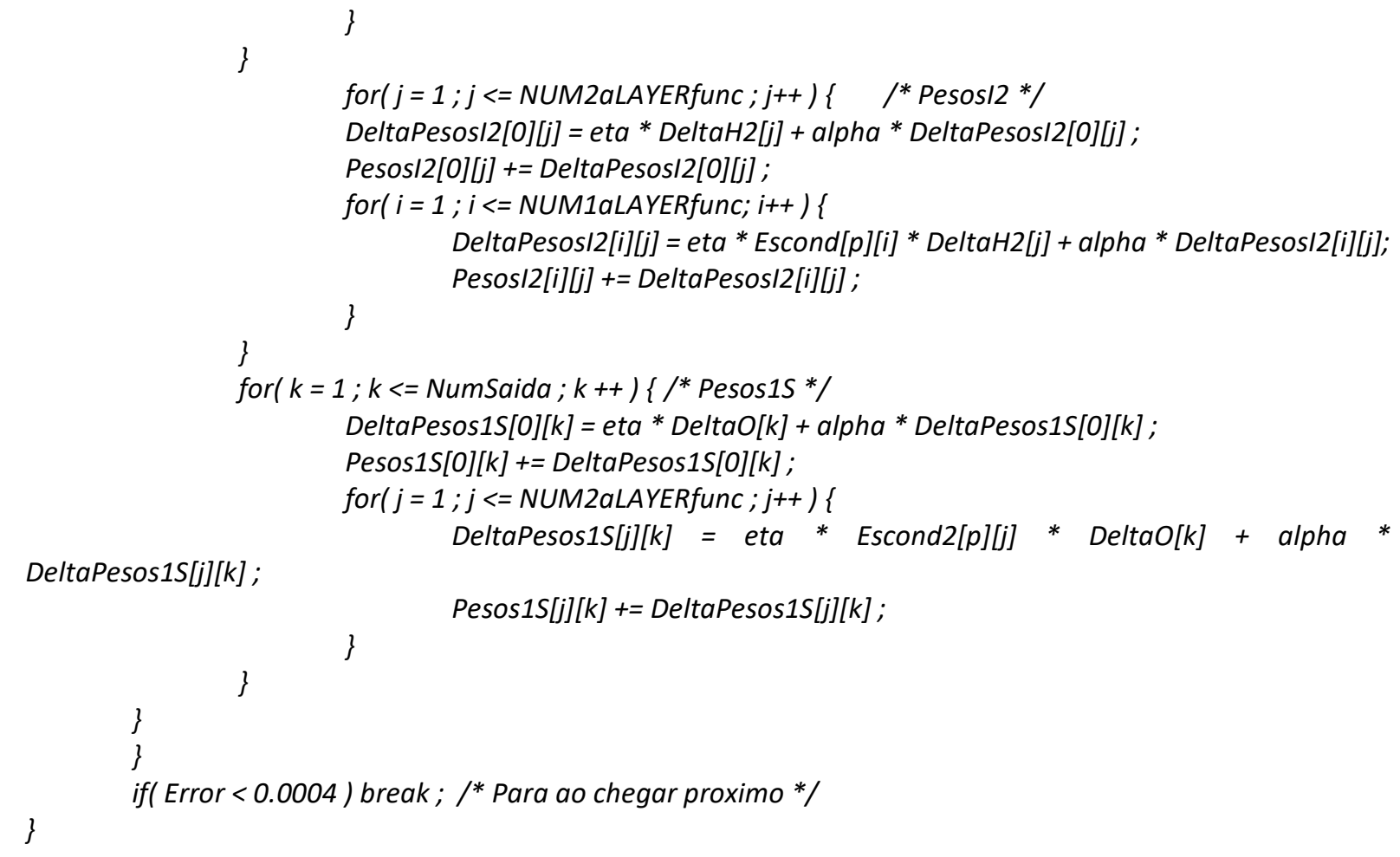




\section{APÊNDICE C - Extratos do SW criado para análise com lógica fuzzy}

\section{Função de pertinência triangular}

/* $^{*}$

Função de pertinencia triangulo

recebe $R$ de entrada, e os valores de inicio, pico e fim da função

retorna o peso segundo a função

se ini=pico, trapezio até 0 .

se pico=fim, trapezio até infinito.

*/

float funcao_triang(float $R$, float ini, float pico, float fim)

\{

float Rout=0;

if(ini==pico)

\{

if(R>fim)

Rout $=0$;

else if $(R<=$ pico $)$

Rout=1;

else

Rout=R*(1/(pico-fim))-(fim/(pico-fim));

\}

else if(fim==pico)

\{

if(R<ini)

Rout=0;

else if $(R<=$ pico)

Rout $=R^{*}(1 /($ pico-ini))-(ini/(pico-ini));

else

Rout=1;

\}

else if $(R<$ ini)

Rout=0;

else if( $R>$ fim)

Rout=0;

else if $(R<=p i c o)$

Rout=(R/(pico-ini))-(ini/(pico-ini));

else

return Rout;

Rout=(R/(pico-fim))-(fim/(pico-fim));

\}

\section{Função de pertinência gaussiana}

/*

Função de pertinencia gaussiana

recebe $R$ de entrada, e os valores de inicio, pico e fim da função

retorna o peso segundo a função gaussiana

*/

float funcao_gauss(float $R$, float ini, float pico, float fim)

\{

float Rout=0;

Rout=(R-pico)/(fim-ini);

Rout $=-4{ }^{*}$ Rout ${ }^{*}$ Rout;

Rout=exp(Rout);

return Rout; 


\section{Cálculo das funções de pertinência}

// lê a resistencia, a capacitancia e o $\mathrm{SOH}$ de cada dado e calcula todas as funções de pertinencia while $(c<c \max )$

\{

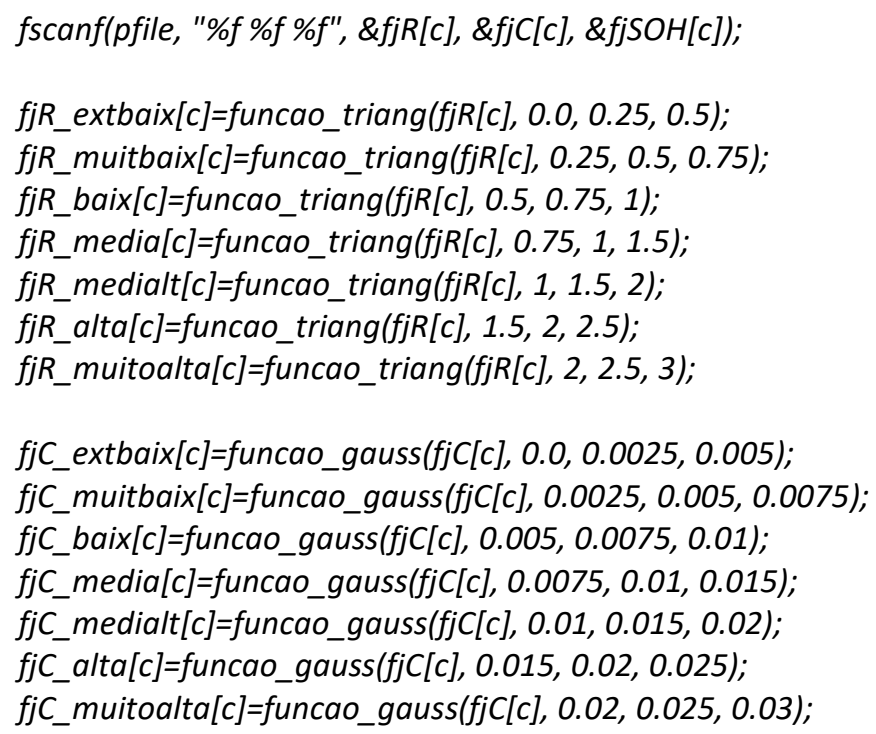

//printf("\%f \%f \%f \%f \%f \%f \%f \%f|n", fjR[c], fjR_extbaix[c], fjR_muitbaix[c], fjR_baix[c], fjR_media[c], fjR_medialt [c], fjR_alta[c], fjR_muitoalta[c]);

\section{Cálculo do SOH estimado para o Sugeno de ordem 0}

//calculo da previsão segundo a tabela de regras atual while (c<cmax)

fjres[c]=minimo(fjR_extbaix[c],fjC_extbaix[c] ${ }^{*}$ ftabela[0];

fjres $[c]=f j r e s[c]+$ minimo(fjR_extbaix[c],fjC_muitbaix[c])*ftabela[3];

fjres $[c]=f j r e s[c]+$ minimo(fjR_extbaix[c],fjc_baix[c] $)^{*}$ ftabela[1];

fjres $[c]=f j r e s[c]+$ minimo(fjR_extbaix[c],fjc_media[c])*ftabela[2];

fjres $[c]=$ fjres $[c]+$ minimo(fjR_extbaix[c],fjC_medialt $[c]) *$ ftabela[6];

fjres $[c]=f j r e s[c]+m i n i m o\left(f j R \_e x t b a i x[c], f j c \_a l t a[c]\right) * f t a b e l a[5]$;

fjres $[c]=f j r e s[c]+$ minimo(fjR_extbaix[c],fjc_muitoalta[c] $)^{*}$ ftabela[4];

fjres $[c]=f j r e s[c]+$ minimo(fjR_muitbaix $[c], f j C \_$extbaix $\left.[c]\right)^{*}$ ftabela $[8]$;

fjres $[c]=f j r e s[c]+$ minimo(fjR_muitbaix[c],fjC_muitbaix[c] $)^{*}$ ftabela[9];

fjres $[c]=f j r e s[c]+$ minimo(fjR_muitbaix[c],fjc_baix[c] $)^{*}$ ftabela[10];

fjres $[c]=$ fjres $[c]+$ minimo(fjR_muitbaix[c],fji__media[c])*ftabela[11];

fjres $[c]=f j r e s[c]+$ minimo(fjR_muitbaix[c],fjC_medialt[c])*ftabela[16];

fjres[c]=fjres[c]+minimo(fjR_muitbaix[c],fjC_alta[c])*ftabela[12]; 
fjres[c]=fjres[c]+minimo(fjR_muitbaix[c],fjC_muitoalta[c] $)^{*}$ ftabela[17]; fjres $[c]=f j r e s[c]+$ minimo(fjR_baix[c],fjC_extbaix[c] ${ }^{*}$ ftabela[18]; fjres $[c]=f j r e s[c]+$ minimo(fjR_baix[c],fjC_muitbaix[c] $)^{*}$ ftabela[13]; fjres[c]=fjres[c]+minimo(fjR_baix[c],fjc_baix[c])*ftabela[14]; fjres[c]=fjres[c]+minimo(fjR_baix[c],fjC_media[c])*ftabela[7]; fjres $[c]=f j r e s[c]+m i n i m o\left(f j R \_b a i x[c], f j C \_\right.$medialt $\left.[c]\right) * f t a b e l a[19] ;$ fjres $[c]=f j r e s[c]+$ minimo(fjR_baix[c],fjc_alta[c] $) * f t a b e l a[15]$; fjres[c]=fjres[c]+minimo(fjR_baix[c],fjC_muitoalta[c])*ftabela[22]; fjres[c]=fjres[c]+minimo(fjR_media[c],fjC_extbaix[c])*ftabela[21]; fjres $[c]=f j r e s[c]+$ minimo(fjR_media[c],fjC_muitbaix[c])*ftabela[20]; fjres $[c]=$ fjres $[c]+$ minimo(fjR_media[c],fjc_baix $[c]) *$ ftabela[23]; fjres $[c]=f j r e s[c]+$ minimo(fjR_media[c],fjc_media[c])*ftabela[26]; fjres[c]=fjres[c]+minimo(fjR_media[c],fjC_medialt[c])*ftabela[27]; fjres $[c]=f j r e s[c]+$ minimo(fjR_media[c],fjC_alta[c])*ftabela[25]; fjres $[c]=f j r e s[c]+$ minimo(fjR_media[c],fjC_muitoalta[c] $)^{*}$ ftabela[24]; fjres $[c]=f j r e s[c]+$ minimo(fjR_medialt $[c], f j\left[\_\right.$extbaix $\left.[c]\right) * f t a b e l a[28]$; fjres $[c]=$ fjres $[c]+$ minimo(fjR_medialt $[c]$,fjC_muitbaix[c])*ftabela[29]; fjres $[c]=f j r e s[c]+$ minimo(fjR_medialt $[c], f j C \_$baix $\left.[c]\right) *$ ftabela[30]; fjres $[c]=f j r e s[c]+$ minimo(fjR_medialt $[c], f j\left[\_\right.$media $\left.[c]\right) * f t a b e l a[33]$; fjres $[c]=f j r e s[c]+$ minimo(fjR_medialt $[c], f j C_{-}$medialt $\left.[c]\right) * f t a b e l a[31]$; fjres $[c]=f j r e s[c]+m i n i m o\left(f j R \_\right.$medialt $\left.[c], f j C \_a l t a[c]\right) *$ ftabela[32]; fjres $[c]=f j r e s[c]+$ minimo(fjR_medialt $[c], f j C \_$muitoalta $\left.[c]\right) *$ ftabela[34]; fjres[c]=fjres[c]+minimo(fjR_alta[c],fjC_extbaix[c])*ftabela[35]; fjres $[c]=$ fjres $[c]+$ minimo(fjR_alta[c],fjC_muitbaix[c])*ftabela[36]; fjres[c]=fjres[c]+minimo(fjR_alta[c],fjc_baix[c])*ftabela[37]; fjres $[c]=f j r e s[c]+$ minimo(fjR_alta[c],fji__media[c])*ftabela[38]; fjres $[c]=f j r e s[c]+$ minimo $\left(f j R_{-} a l t a[c], f j C \_\right.$medialt $\left.[c]\right) * f t a b e l a[39]$; fjres $[c]=f j r e s[c]+$ minimo(fjR_alta[c],fjC_alta[c])*ftabela[40]; fjres $[c]=$ fjres $[c]+$ minimo(fjR_alta[c],fjC_muitoalta[c] $)^{*}$ ftabela[41]; fjres $[c]=$ fjres $[c]+$ minimo(fjR_muitoalta[c],fjC_extbaix[c])*ftabela[42]; fjres[c]=fjres[c]+minimo(fjR_muitoalta[c],fjC_muitbaix[c])*ftabela[43]; fjres $[c]=f j r e s[c]+$ minimo(fjR_muitoalta[c],fjj_baix[c])*ftabela[44]; fjres $[c]=f j r e s[c]+$ minimo(fjR_muitoalta $[c], f j C_{-}$media $\left.[c]\right) *$ ftabela $[45]$; fjres $[c]=f j r e s[c]+$ minimo(fjR_muitoalta[c],fjc_medialt $[c]) * f t a b e l a[46]$; fjres[c]=fjres[c]+minimo(fjR_muitoalta[c],fjC_alta[c])*ftabela[47]; fjres $[c]=f j r e s[c]+$ minimo(fjR_muitoalta $[c], f j C_{-}$muitoalta[c] $)^{*}$ ftabela[48];

soma=minimo(fjR_extbaix[c],fjC_extbaix[c]); soma $=$ soma+minimo(fjR_extbaix[c],fjC_muitbaix[c]); soma $=$ soma+minimo(fjR_extbaix $\left.[c], f j C \_b a i x[c]\right)$; soma $=$ soma+minimo(fjR_extbaix $[c], f j C \_$media $\left.[c]\right)$; soma $=$ soma+minimo(fjR_extbaix[c],fjC_medialt $[c])$; soma=soma+minimo(fjR_extbaix[c],fjC_alta[c]); soma $=$ soma+minimo(fjR_extbaix[c],fjC_muitoalta[c]); soma=soma+minimo(fjR_muitbaix[c],fjC_extbaix[c]); soma $=$ soma+minimo(fjR_muitbaix $[c], f j C \_$muitbaix $\left.[c]\right)$; soma $=$ soma+minimo(fjR_muitbaix [c],fjC_baix[c]); soma $=$ soma+minimo(fjR_muitbaix[c],fjC_media[c]); soma $=$ soma+minimo(fjR_muitbaix $[c], f j C \_$medialt $\left.[c]\right)$; soma $=$ soma+minimo(fjR_muitbaix[c],fjC_alta[c]); soma $=$ soma+minimo(fji_muitbaix[c],fjC_muitoalta[c]); soma $=$ soma+minimo(fjR_baix $[c], f j C \_$extbaix $\left.[c]\right)$; 


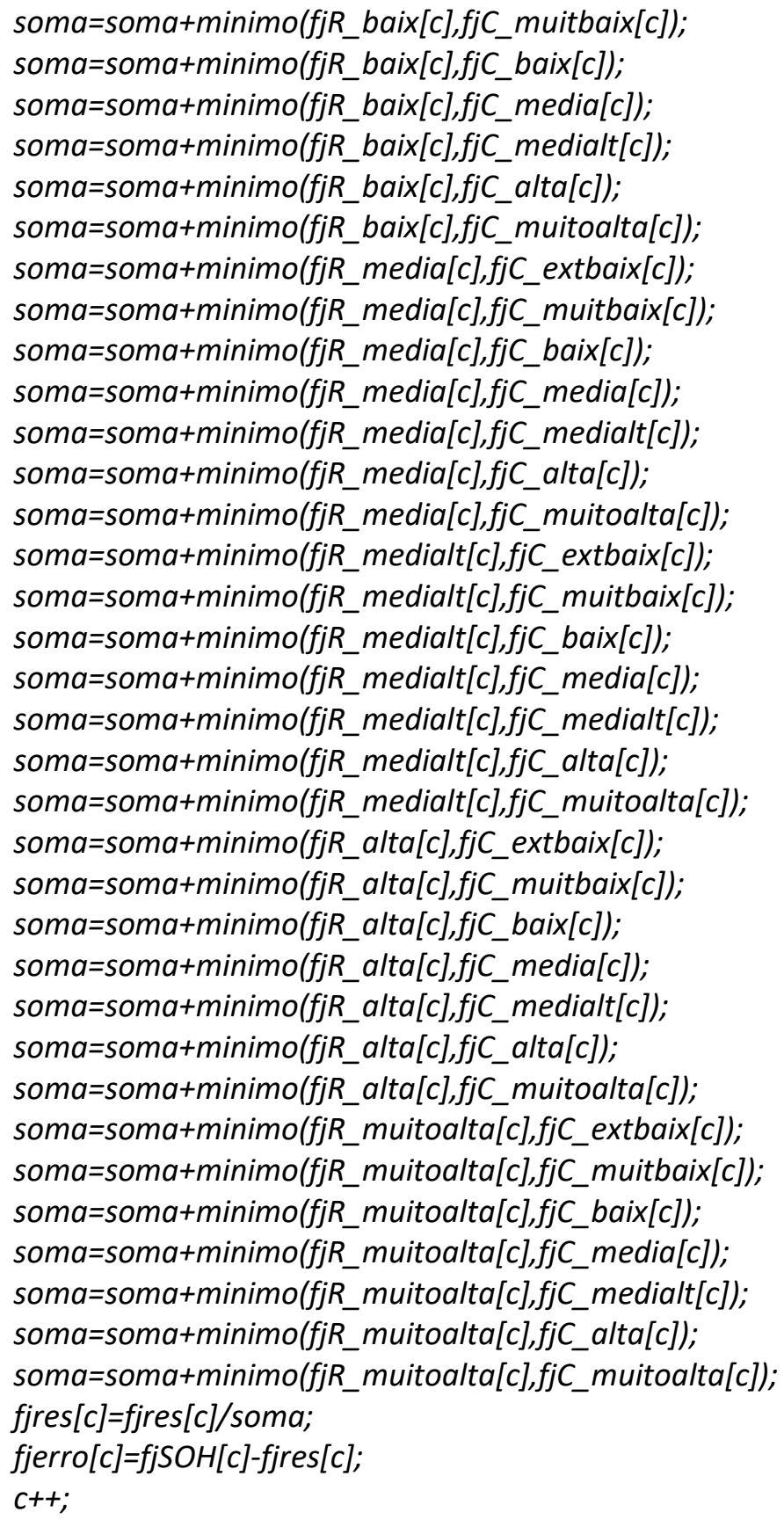




\section{APÊNDICE D - Extratos do SW criado para análise com algoritmo adaptativo.}

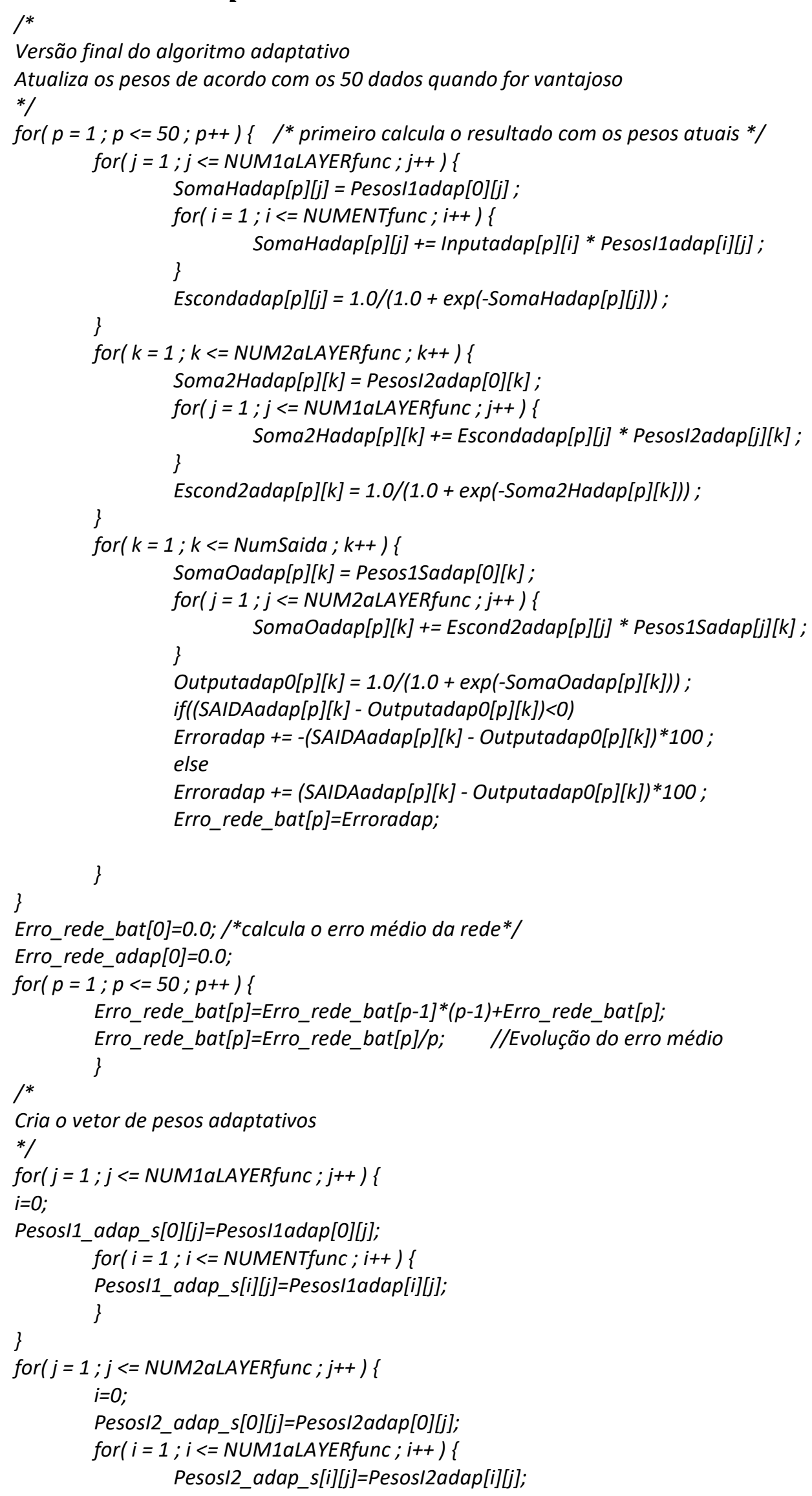




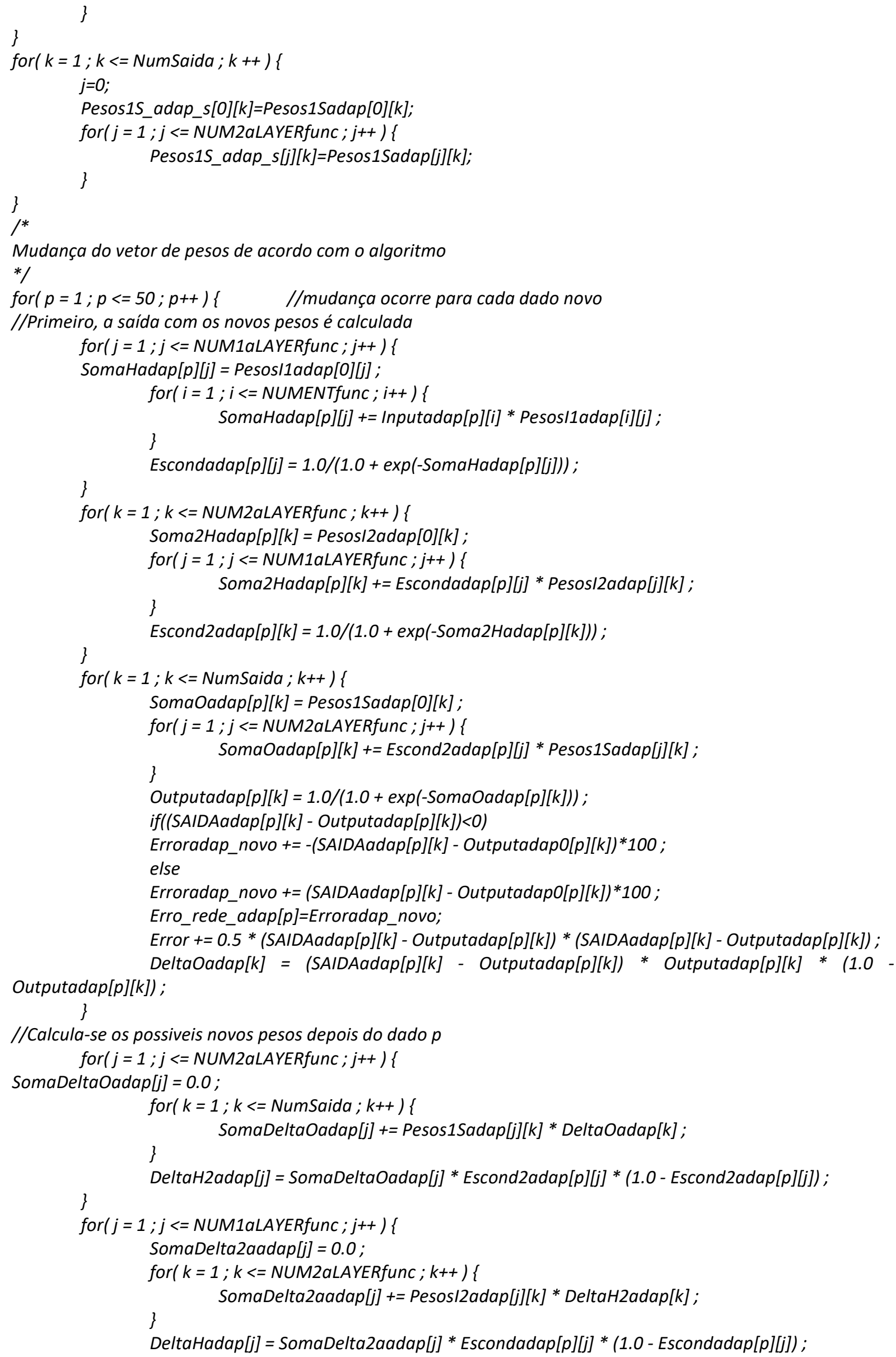


\}

for $(j=1 ; j<=$ NUM1aLAYERfunc $; j++)\{$

DeltaPesosI1adap[0][j] = etaadap * DeltaHadap[j] + alphaadap * DeltaPesosI1adap[0][j] ;

Pesosl1adap[0][j] += DeltaPesos/1adap[0][j] ;

for $(i=1 ; i<=$ NUMENTfunc $; i++)\{$

DeltaPesos/1adap $[i][j]=$ etaadap $*$ Inputadap[p][i] * DeltaHadap[j] + alphaadap *

DeltaPesos/1adap[i][j];

PesosI1adap[i][j] += DeltaPesosI1adap[i][j] ;

\}

for $(j=1 ; j<=$ NUM2aLAYERfunc $; j++)\{$

DeltaPesos/2adap[0][j] = etaadap * DeltaH2adap[j] + alphaadap * DeltaPesos/2adap[0][j] ;

Pesos/2adap[O][j] += DeltaPesos/2adap[0][j] ;

for $(i=1 ; i<=$ NUM1aLAYERfunc; $i++)\{$

DeltaPesos/2adap[i][j];

DeltaPesos/2adap[i][j] = etaadap * Escondadap[p][i] * DeltaH2adap[j] + alphaadap *

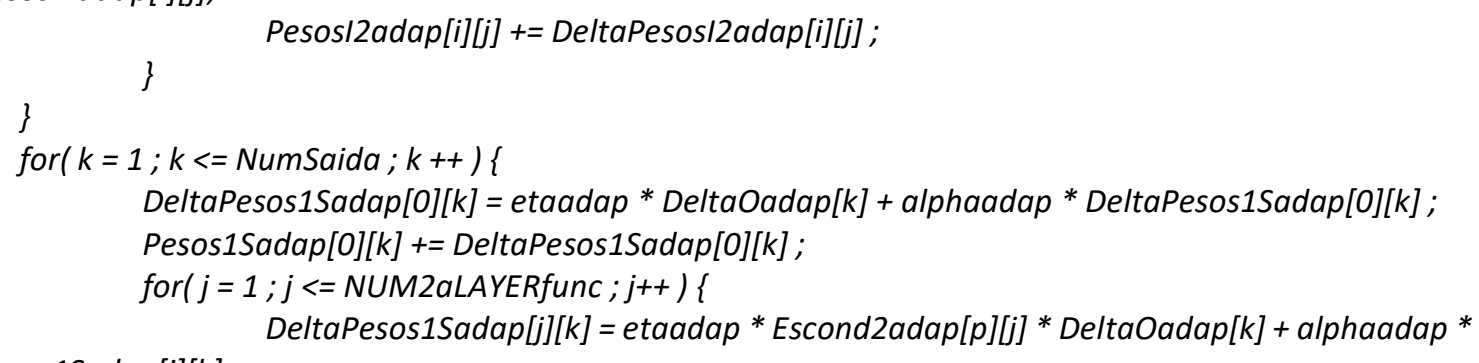

//Define-se o novo erro médio

Erro_rede_adap $[p]=E r r o \_r e d e \_a d a p[p-1]^{*}(p-1)+E r r o \_r e d e \_b a t[p] ;$

Erro_rede_adap[p]=Erro_rede_adap[p]/p; $\quad$ //Evolução do erro médio

//Se o novo erro é menor que o erro da rede inicial, os pesos são atualizados

if(Erro_rede_bat[p]-Erro_rede_adap[p]<Erro_rede_bat[p-1]-Erro_rede_adap[p-1])\{

for $(j=1 ; j<=$ NUM1aLAYERfunc $; j++)\{$

$i=0$;

Pesos/1adap[0][j]=Pesos/1_adap_s[0][j];

for $(i=1 ; i<=$ NUMENTfunc $; i++)\{$

PesosI1adap[i][j]=PesosI1_adap_s[i][j];

\}

\}

for $(j=1 ; j<=$ NUM2aLAYERfunc $; j++)\{$

$i=0$;

Pesos/2adap[0][j]=Pesos/2_adap_s[0][j];

for $(i=1 ; i<=$ NUM1aLAYERfunc $; i++)\{$

\}

Pesos/2adap[i][j]=Pesos/2_adap_s[i][j];

\}

for $(k=1 ; k<=$ NumSaida $; k++)\{$

$j=0$;

Pesos1Sadap[0][k]=Pesos1S_adap_s[0][k];

for $(j=1 ; j<=$ NUM2aLAYERfunc $; j++)\{$

Pesos1Sadap[j][k]=Pesos1S_adap_s[j][k];

\}

\}

\} 


\section{APÊNDICE E - Extratos do SW criado para análise usando método linear}

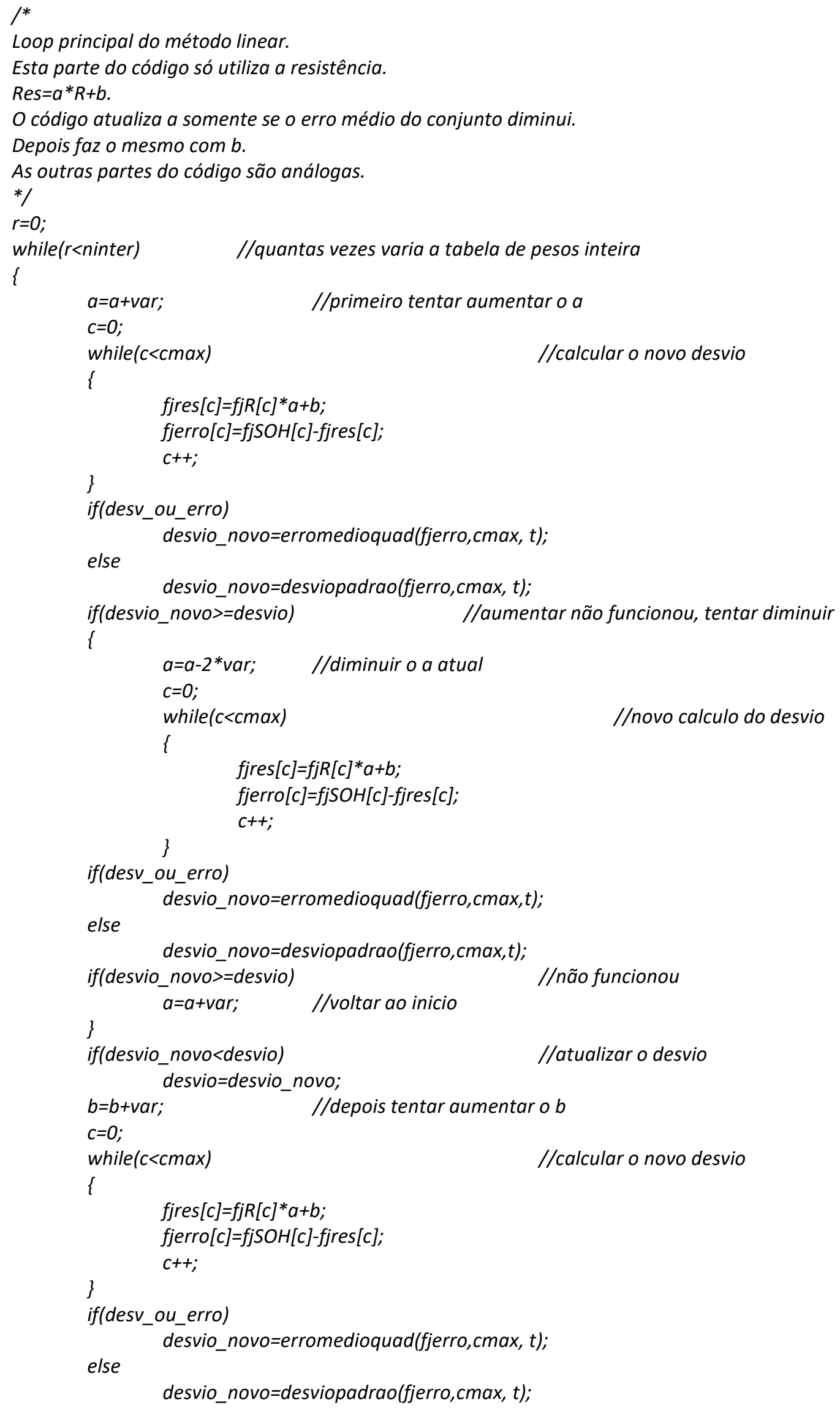




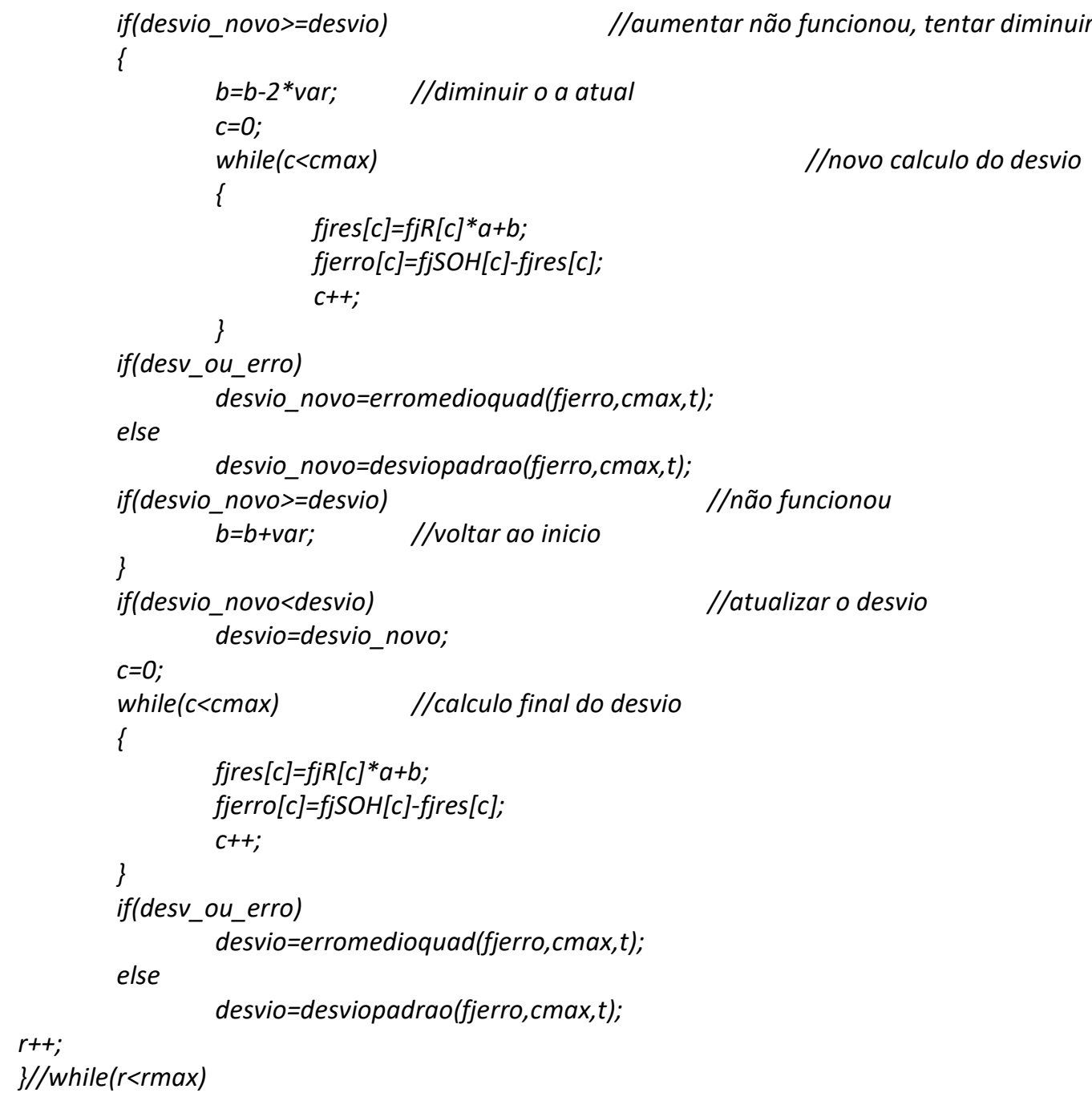




\section{APÊNDICE F - Pesos da rede neural 9x5}

Tabela 22 - Pesos da rede neural 9x5.

\begin{tabular}{|c|c|c|c|c|c|}
\hline \multicolumn{2}{|c|}{ Pesos para camada inicial } & \multicolumn{2}{|c|}{$\begin{array}{l}\text { Pesos para camada } \\
\text { interna }\end{array}$} & \multicolumn{2}{|c|}{ Pesos para camada de saída } \\
\hline PesosI1[0][1] & $-61,7903$ & Pesos/2[0][1] & $-39,4417$ & Pesos1S[0][1] & $-0,36222$ \\
\hline Pesosl1[1][1] & 34,2397 & PesosI2[1][1] & $-16,1667$ & Pesos1S[1][1] & 0,67579 \\
\hline PesosI1[2][1] & 34,01094 & PesosI2[2][1] & 1,601394 & Pesos1S[2][1] & 3,921868 \\
\hline PesosI1[0][2] & 0,280688 & Pesosl2[3][1] & $-8,00809$ & Pesos1S[3][1] & 0,568782 \\
\hline PesosI1[1][2] & $-5,13499$ & Pesosl2[4][1] & 27,66003 & Pesos1S[4][1] & 2,990406 \\
\hline PesosI1[2][2] & 51,42062 & Pesosl2[5][1] & $-33,1832$ & Pesos1S[5][1] & $-4,31418$ \\
\hline PesosI1[0][3] & $-81,6077$ & Pesosl2[6][1] & 34,61749 & & \\
\hline PesosI1[1][3] & 37,00368 & PesosI2[7][1] & 10,89328 & & \\
\hline Pesos/1[2][3] & $-2,52399$ & Pesos/2[8][1] & 3,198584 & & \\
\hline PesosI1[0][4] & 6,115082 & Pesosl2[9][1] & 73,09585 & & \\
\hline PesosI1[1][4] & $-3,0524$ & PesosI2[0][2] & $-46,3685$ & & \\
\hline PesosI1[2][4] & $-222,119$ & Pesosl2[1][2] & 72,85651 & & \\
\hline PesosI1[0][5] & 23,10449 & PesosI2[2][2] & 1,508359 & & \\
\hline Pesos/1[1][5] & $-5,15878$ & Pesos/2[3][2] & 26,68519 & & \\
\hline PesosI1[2][5] & 24,37525 & PesosI2[4][2] & 84,71968 & & \\
\hline PesosI1[0][6] & 10,21942 & Pesosl2[5][2] & $-51,7277$ & & \\
\hline PesosI1[1][6] & $-9,64069$ & Pesosl2[6][2] & 3,772577 & & \\
\hline PesosI1[2][6] & 124,3874 & PesosI2[7][2] & $-3,09644$ & & \\
\hline PesosI1[0][7] & 40,83768 & PesosI2[8][2] & $-1,74347$ & & \\
\hline PesosI1[1][7] & $-35,4888$ & Pesosl2[9][2] & 3,239309 & & \\
\hline PesosI1[2][7] & $-17,363$ & Pesos/2[0][3] & 16,87319 & & \\
\hline PesosI1[0][8] & 20,37579 & PesosI2[1][3] & 21,29228 & & \\
\hline PesosI1[1][8] & $-21,1929$ & PesosI2[2][3] & $-1,27536$ & & \\
\hline Pesosl1[2][8] & $-16,6286$ & Pesos/2[3][3] & 3,705578 & & \\
\hline Pesos/1[0][9] & 61,9107 & PesosI2[4][3] & $-12,6808$ & & \\
\hline Pesos/1[1][9] & $-43,9442$ & Pesosl2[5][3] & 8,185886 & & \\
\hline \multirow[t]{13}{*}{ PesosI1[2][9] } & $-15,7758$ & Pesosl2[6][3] & $-19,3325$ & & \\
\hline & & PesosI2[7][3] & $-5,7221$ & & \\
\hline & & Pesosl2 [8][3] & $-3,03526$ & & \\
\hline & & Pesos/2[9][3] & $-114,977$ & & \\
\hline & & Pesos/2[0][4] & $-2,09829$ & & \\
\hline & & Pesos/2[1][4] & $-73,2697$ & & \\
\hline & & PesosI2[2][4] & 7,432286 & & \\
\hline & & PesosI2[3][4] & $-4,7329$ & & \\
\hline & & Pesos/2[4][4] & 5,174763 & & \\
\hline & & Pesos/2[5][4] & $-2,80904$ & & \\
\hline & & PesosI2[6][4] & $-2,70199$ & & \\
\hline & & PesosI2[7][4] & 1,441699 & & \\
\hline & & PesosI2[8][4] & 0,995654 & & \\
\hline
\end{tabular}




\begin{tabular}{|l|l|l|l|l|l|} 
& & PesosI2[9][4] & $-0,08398$ & & \\
\hline & & PesosI2[0][5] & $-44,6804$ & & \\
\hline & & PesosI2[1][5] & 59,03354 & & \\
\hline & & PesosI2[2][5] & $-2,34564$ & & \\
\hline & & PesosI2[3][5] & 16,9085 & & \\
\hline & & PesosI2[4][5] & 60,6986 & & \\
\hline & & PesosI2[5][5] & $-32,3137$ & & \\
\hline & & PesosI2[6][5] & $-65,0158$ & & \\
\hline & & PesosI2[7][5] & $-1,20419$ & & \\
\hline & & PesosI2[8][5] & $-1,47339$ & & \\
\hline & & PesosI2[9][5] & $-12,4915$ & & \\
\hline
\end{tabular}

Fonte: Autor 


\section{APÊNDICE G - Tabela de regras obtidas para o modelo de Sugeno de ordem 0}

Tabela 23 - Regras do modelo de Sugeno de ordem 0.

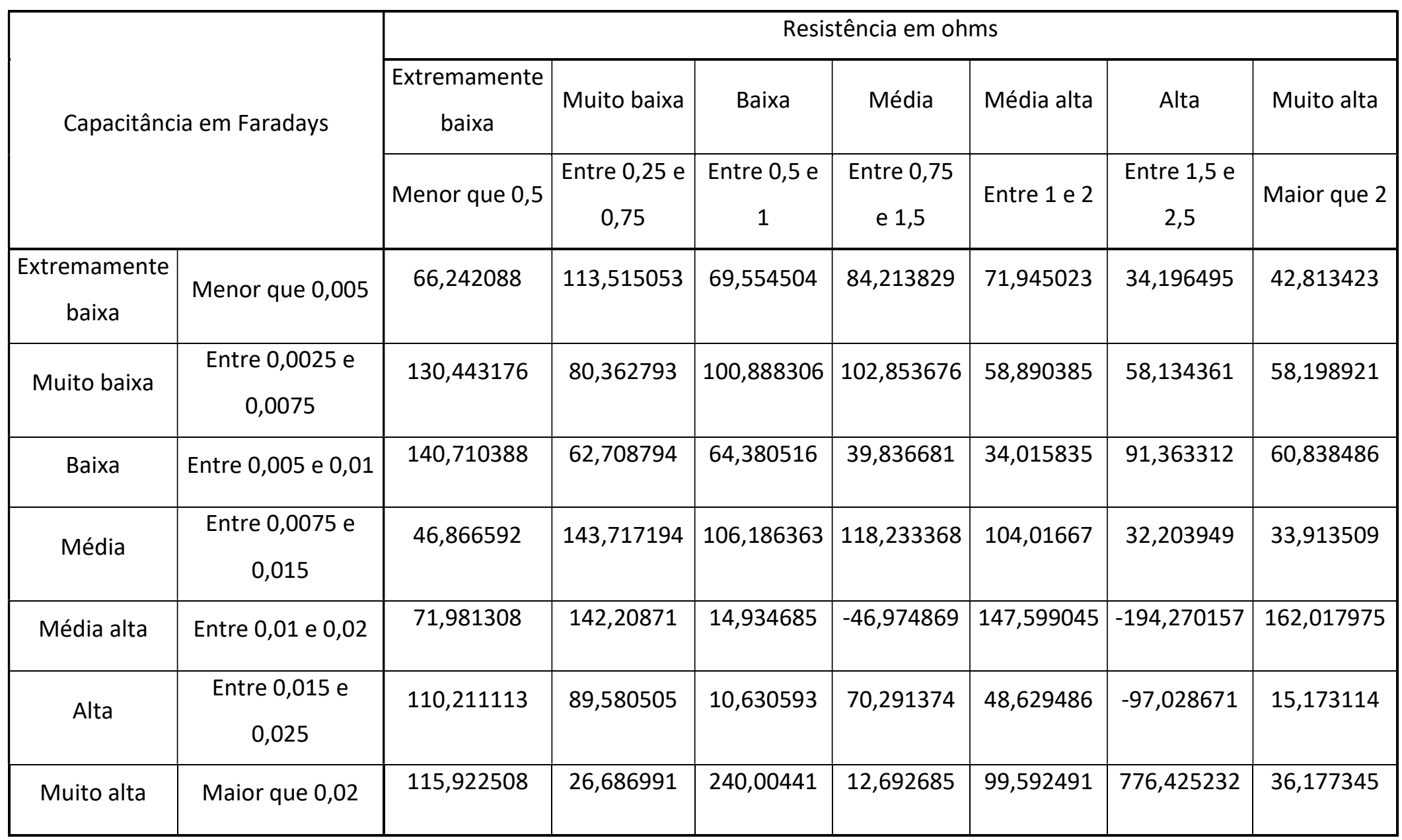




\section{APÊNDICE H - Tabela de regras obtidas para o modelo de Sugeno de ordem 1}

Tabela 24 - Regras do modelo de Sugeno de ordem 1, regras para $\mathrm{R}_{\mathrm{i}}$.

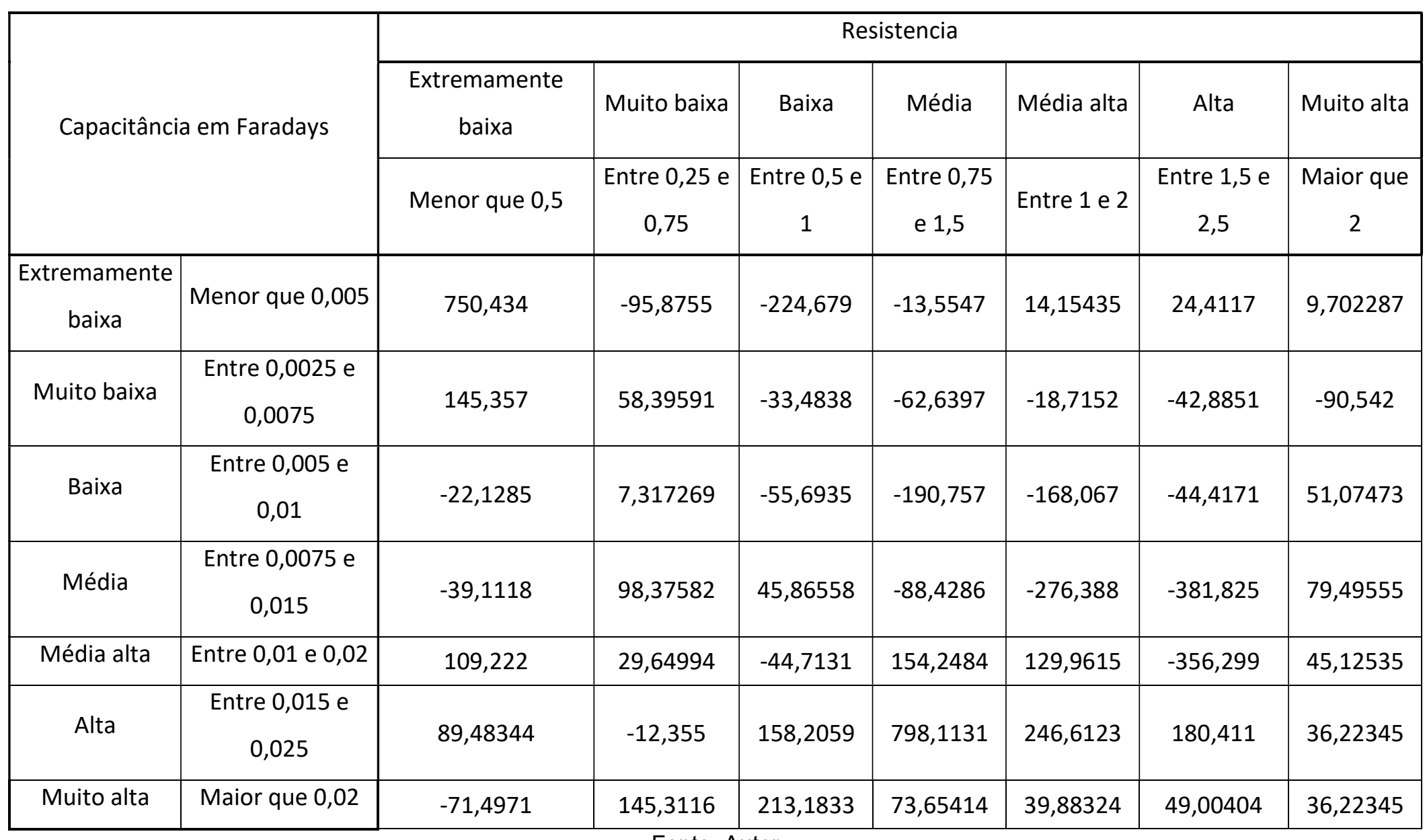

Fonte: Autor 
Tabela 25 - Regras do modelo de Sugeno de ordem 1, regras para $\mathrm{C}_{\mathrm{i}}$.

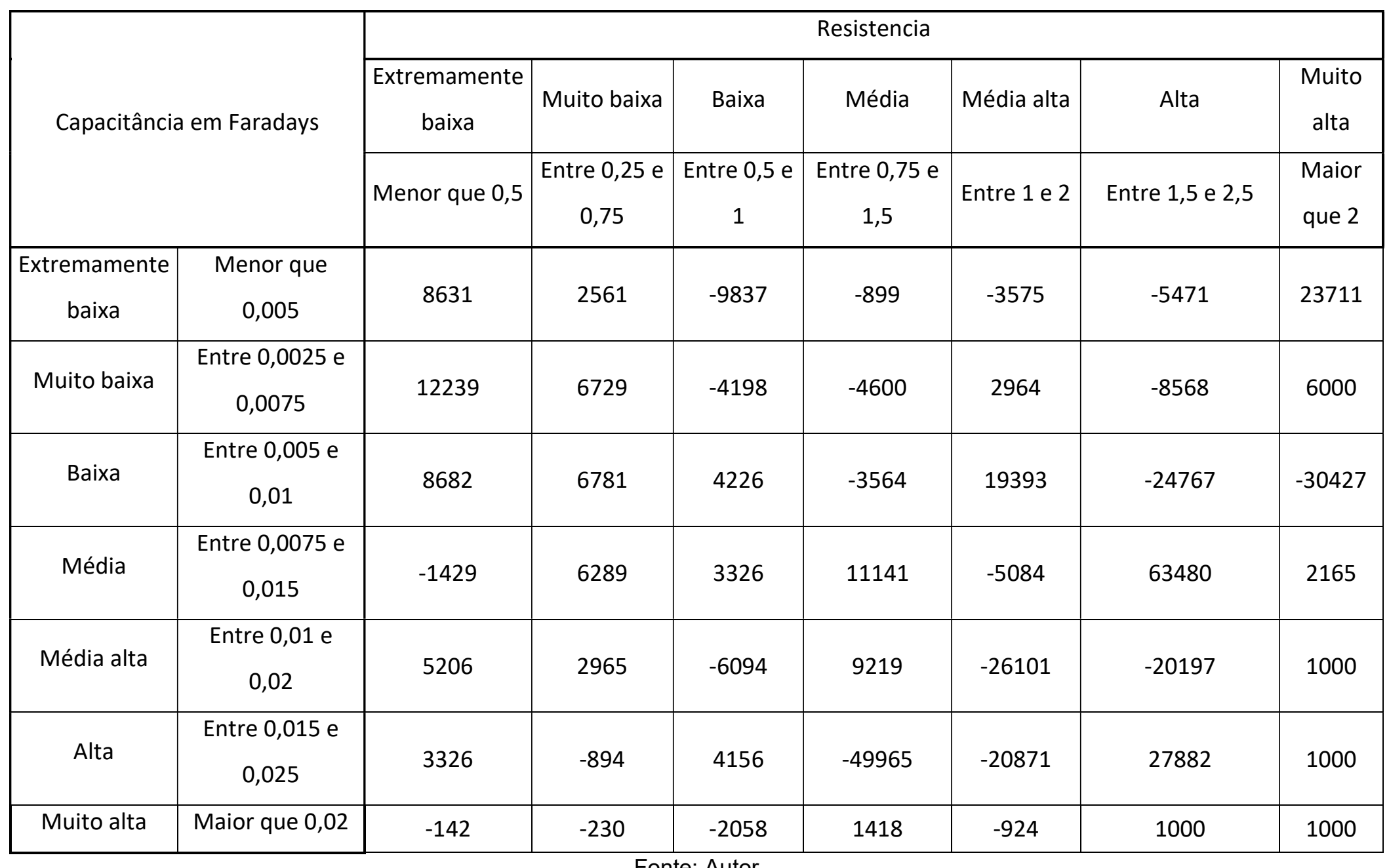


Tabela 26 - Regras do modelo de Sugeno de ordem 1, regras para $b_{i}$.

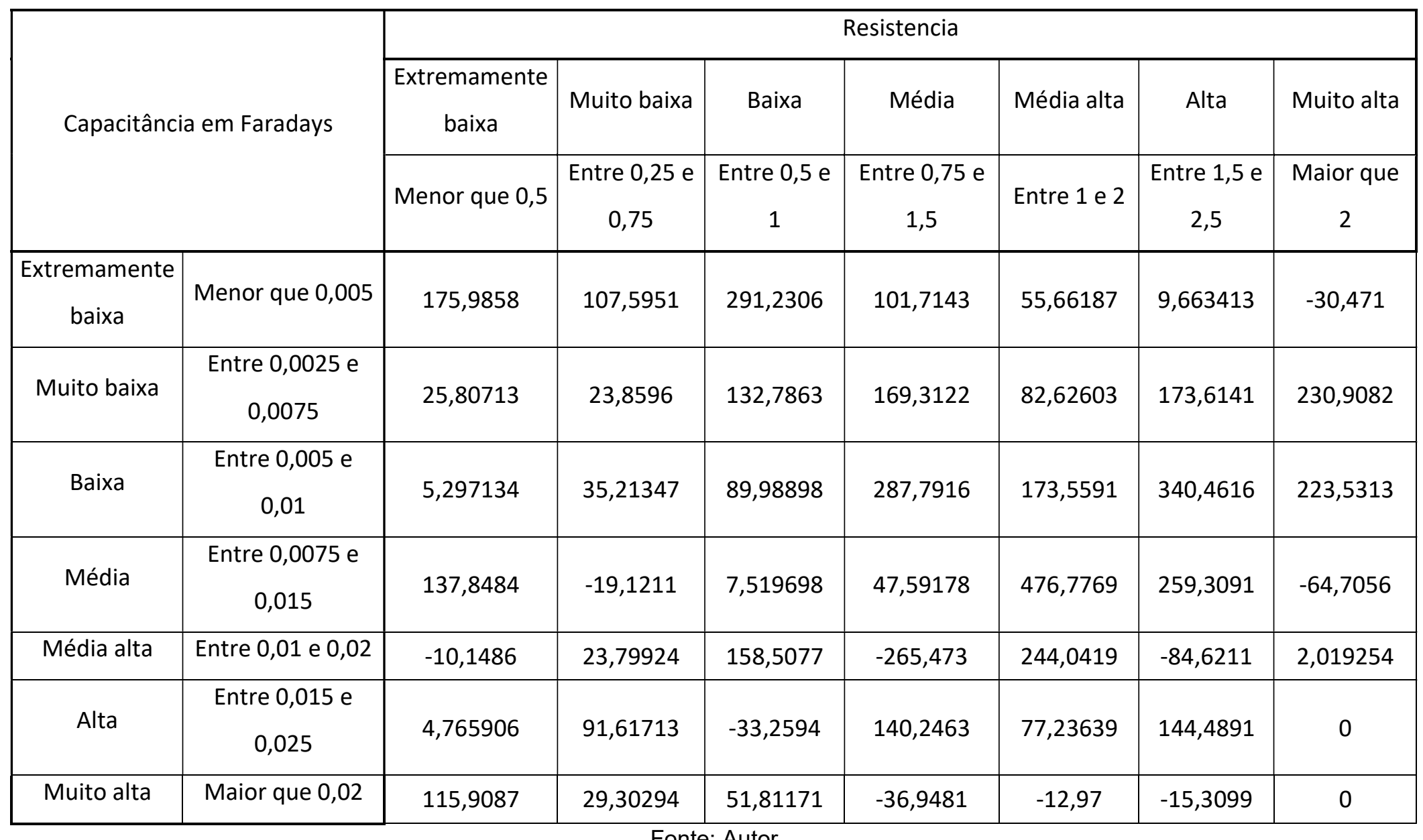

Fonte: Autor 


\section{APENNDICE I - Pesos obtidos para a segunda rede neural do método de melhoramento de EOL.}

Tabela 27 - Pesos da segunda rede neural.

\begin{tabular}{|c|c|c|c|}
\hline \multicolumn{2}{|c|}{ Pesos para camada inicial } & \multicolumn{2}{c|}{ Pesos para camada de saída } \\
\hline PesosI1[0][1] & 95,972503 & Pesos1S[0][1] & $-0,324029$ \\
\hline PesosI1[1][1] & $-138,114413$ & Pesos1S[1][1] & $-35,252237$ \\
\hline PesosI1[2][1] & 11,733063 & Pesos1S[2][1] & $-0,856673$ \\
\hline PesosI1[0][2] & $-2,170303$ & Pesos1S[3][1] & $-5,954368$ \\
\hline PesosI1[1][2] & $-6,285927$ & Pesos1S[4][1] & 4,858648 \\
\hline PesosI1[2][2] & $-0,908209$ & Pesos1S[5][1] & 15,603116 \\
\hline PesosI1[0][3] & 73,228816 & Pesos1S[6][1] & 2,415738 \\
\hline PesosI1[1][3] & $-70,398192$ & Pesos1S[7][1] & 55,598816 \\
\hline PesosI1[2][3] & 43,074535 & Pesos1S[8][1] & 14,340713 \\
\hline PesosI1[0][4] & 46,086164 & Pesos1S[9][1] & $-4,760764$ \\
\hline PesosI1[1][4] & $-34,15526$ & Pesos1S[10][1] & $-28,501614$ \\
\hline PesosI1[2][4] & 425,476742 & Pesos1S[11][1] & 1,688606 \\
\hline PesosI1[0][5] & $-8,725761$ & Pesos1S[12][1] & $-75,957276$ \\
\hline PesosI1[1][5] & 2,958712 & Pesos1S[13][1] & $-2,911083$ \\
\hline PesosI1[2][5] & $-48,88574$ & Pesos1S[14][1] & $-0,688902$ \\
\hline PesosI1[0][6] & 127,540824 & Pesos1S[15][1] & 2,31028 \\
\hline PesosI1[1][6] & $-130,212125$ & Pesos1S[16][1] & $-1,116536$ \\
\hline PesosI1[2][6] & 66,402548 & Pesos1S[17][1] & $-9,391836$ \\
\hline PesosI1[0][7] & 115,78452 & Pesos1S[18][1] & 9,598775 \\
\hline PesosI1[1][7] & $-97,571047$ & Pesos1S[19][1] & $-4,066406$ \\
\hline PesosI1[2][7] & $-227,304975$ & Pesos1S[20][1] & 36,800227 \\
\hline PesosI1[0][8] & 120,35499 & Pesos1S[21][1] & $-18,973737$ \\
\hline PesosI1[1][8] & $-114,169662$ & Pesos1S[22][1] & $-44,929058$ \\
\hline PesosI1[2][8] & $-41,999765$ & Pesos1S[23][1] & 0,080138 \\
\hline PesosI1[0][9] & 337,12661 & Pesos1S[24][1] & 72,25277 \\
\hline PesosI1[1][9] & $-242,395645$ & Pesos1S[25][1] & 1,751879 \\
\hline PesosI1[2][9] & $-6,895002$ & & \\
\hline PesosI1[0][10] & 14,906607 & & \\
\hline PesosI1[1][10] & $-31,076799$ & & \\
\hline PesosI1[2][10] & 34,477918 & & \\
\hline PesosI1[0][11] & 200,087935 & & \\
\hline PesosI1[1][11] & $-154,957786$ & & \\
\hline
\end{tabular}




\begin{tabular}{|c|c|l|l|}
\hline PesosI1[2][11] & $-63,195838$ & & \\
\hline PesosI1[0][12] & 202,578214 & & \\
\hline PesosI1[1][12] & $-189,595338$ & & \\
\hline PesosI1[2][12] & $-18,818559$ & & \\
\hline PesosI1[0][13] & 26,51939 & & \\
\hline PesosI1[1][13] & $-27,206125$ & & \\
\hline PesosI1[2][13] & $-3,221848$ & & \\
\hline PesosI1[0][14] & $-2,416869$ & & \\
\hline PesosI1[1][14] & $-5,955662$ & & \\
\hline PesosI1[2][14] & $-2,50939$ & & \\
\hline PesosI1[0][15] & $-2,746422$ & & \\
\hline PesosI1[1][15] & $-5,563742$ & & \\
\hline PesosI1[2][15] & 5,382628 & & \\
\hline PesosI1[0][16] & $-2,324926$ & & \\
\hline PesosI1[1][16] & $-6,567545$ & & \\
\hline PesosI1[2][16] & $-2,699763$ & & \\
\hline PesosI1[0][17] & 103,796153 & & \\
\hline PesosI1[1][17] & $-129,377382$ & & \\
\hline PesosI1[2][17] & $-47,471761$ & & \\
\hline PesosI1[0][18] & 70,208358 & & \\
\hline PesosI1[1][18] & $-88,89779$ & & \\
\hline PesosI1[2][18] & 165,087645 & & \\
\hline PesosI1[0][19] & $-1,027897$ & & \\
\hline PesosI1[1][19] & $-10,597628$ & & \\
\hline PesosI1[2][19] & $-3,720547$ & & \\
\hline PesosI1[0][20] & 39,657073 & & \\
\hline PesosI1[1][20] & $-58,714255$ & & \\
\hline PesosI1[2][20] & $-12,653011$ & & \\
\hline PesosI1[0][21] & 32,134449 & & \\
\hline PesosI1[1][21] & $-25,558412$ & & \\
\hline PesosI1[2][21] & $-562,738608$ & & \\
\hline PesosI1[0][22] & 248,532532 & & \\
\hline PesosI1[1][22] & $-211,545641$ & & \\
\hline PesosI1[2][22] & 329,356347 & & \\
\hline PesosI1[0][23] & $-2,419807$ & & \\
\hline PesosI1[1][23] & $-4,927977$ & & \\
\hline PesosI1[2][23] & $-2,104133$ & & \\
\hline PesosI1[0][24] & 321,663367 & & \\
\hline PesosI1[1][24] & $-301,271333$ & & \\
\hline
\end{tabular}




\begin{tabular}{|c|c|l|l|}
\hline PesosI1[2][24] & $-13,043971$ & & \\
\hline PesosI1[0][25] & $-340,187903$ & & \\
\hline PesosI1[1][25] & 216,191774 & & \\
\hline PesosI1[2][25] & 2,866445 & & \\
\hline
\end{tabular}

Fonte: Autor 


\section{APÊNDICE J - Pesos obtidos para a terceira rede neural do método de melhoramento de EOL.}

Tabela 28 - Pesos da terceira rede neural.

\begin{tabular}{|c|c|c|c|c|c|}
\hline \multicolumn{2}{|c|}{ Pesos para camada inicial } & \multicolumn{2}{|c|}{ Pesos para camada interna } & \multicolumn{2}{|c|}{ Pesos para camada de saída } \\
\hline Pesosl1[0][1] & 15,534962 & Pesosl2[0][1] & $-36,744452$ & Pesos1S[0][1] & $-25,699599$ \\
\hline Pesosl1[1][1] & $-7,145164$ & Pesosl2[1][1] & $-6,579665$ & Pesos1S[1][1] & 25,915149 \\
\hline Pesosl1[2][1] & 10,257556 & Pesosi2[2][1] & 40,554723 & Pesos1S[2][1] & 25,918824 \\
\hline Pesosl1[3][1] & $-9,445191$ & Pesosl2[3][1] & 88,113629 & & \\
\hline Pesosl1[4][1] & 1,212491 & Pesosi2[4][1] & 29,236552 & & \\
\hline Pesosl1[0][2] & 2,073742 & Pesosl2[5][1] & 18,149275 & & \\
\hline Pesosl1[1][2] & 29,577284 & Pesosi2[6][1] & $-46,745236$ & & \\
\hline Pesosl1[2][2] & 2,270529 & Pesosi2[7][1] & $-41,124444$ & & \\
\hline Pesosl1[3][2] & $-48,215026$ & Pesosl2[8][1] & $-82,095923$ & & \\
\hline Pesosl1[4][2] & $-0,682035$ & Pesosi2[9][1] & 48,275225 & & \\
\hline Pesosl1[0][3] & 0,446169 & PesosI2[10][1] & 28,757174 & & \\
\hline Pesosl1[1][3] & 59,456065 & Pesosi2[0][2] & $-3,20305$ & & \\
\hline Pesosl1[2][3] & $-2,435603$ & Pesosl2[1][2] & 19,452097 & & \\
\hline Pesosl1[3][3] & $-103,649053$ & Pesosl2[2][2] & $-18,304784$ & & \\
\hline Pesosl1[4][3] & 9,898563 & Pesosl2[3][2] & $-29,171596$ & & \\
\hline Pesosl1[0][4] & 62,560633 & Pesos/2[4][2] & 30,0321 & & \\
\hline Pesosl1[1][4] & $-43,291508$ & Pesosl2[5][2] & 45,47485 & & \\
\hline Pesosl1[2][4] & 17,87446 & Pesosl2[6][2] & $-41,574954$ & & \\
\hline Pesosl1[3][4] & $-17,062553$ & Pesosl2[7][2] & $-36,644281$ & & \\
\hline Pesosl1[4][4] & $-8,734034$ & Pesosl2[8][2] & 30,816271 & & \\
\hline Pesosl1[0][5] & 36,37777 & Pesosl2[9][2] & 47,901926 & & \\
\hline Pesosl1[1][5] & $-10,368285$ & PesosI2[10][2] & 0,679118 & & \\
\hline Pesosl1[2][5] & 25,932433 & & & & \\
\hline Pesosl1[3][5] & $-37,416801$ & & & & \\
\hline Pesosl1[4][5] & $-3,455386$ & & & & \\
\hline Pesosl1[0][6] & $-56,925067$ & & & & \\
\hline Pesosl1[1][6] & 27,53069 & & & & \\
\hline Pesosl1[2][6] & $-26,094798$ & & & & \\
\hline Pesosl1[3][6] & 35,622514 & & & & \\
\hline Pesosl1[4][6] & $-0,617971$ & & & & \\
\hline Pesosl1[0][7] & $-40,519731$ & & & & \\
\hline Pesosl1[1][7] & 1,124078 & & & & \\
\hline Pesosl1[2][7] & $-24,514711$ & & & & \\
\hline
\end{tabular}




\begin{tabular}{|c|c|l|l|l|l|}
\hline PesosI1[3][7] & 44,963145 & & & & \\
\hline PesosI1[4][7] & 6,595631 & & & & \\
\hline PesosI1[0][8] & 65,157298 & & & & \\
\hline PesosI1[1][8] & $-73,210071$ & & & & \\
\hline PesosI1[2][8] & 18,130531 & & & & \\
\hline PesosI1[3][8] & 52,810427 & & & & \\
\hline PesosI1[4][8] & $-19,538961$ & & & & \\
\hline PesosI1[0][9] & 92,835524 & & & & \\
\hline PesosI1[1][9] & $-62,681475$ & & & & \\
\hline PesosI1[2][9] & 24,156769 & & & & \\
\hline PesosI1[3][9] & $-12,489138$ & & & & \\
\hline PesosI1[4][9] & $-13,207605$ & & & & \\
\hline PesosI1[0][10] & 22,425751 & & & & \\
\hline PesosI1[1][10] & $-39,477559$ & & & & \\
\hline PesosI1[2][10] & $-0,059391$ & & & & \\
\hline PesosI1[3][10] & 34,191962 & & & & \\
\hline PesosI1[4][10] & $-2,978487$ & & & & \\
\hline
\end{tabular}

Fonte: Autor 


\section{APÊNDICE K - Relação dos modelos de baterias utilizados.}

Tabela 29 - Relação dos modelos de baterias utilizados.

\begin{tabular}{|c|c|}
\hline Número da Bateria & Modelo \\
\hline 1 & HCT \\
\hline 2 & Expower 59 \\
\hline 3 & HCT \\
\hline 4 & HCT \\
\hline 5 & HCT \\
\hline 6 & HCT \\
\hline 7 & Expower 57 \\
\hline 8 & HCT \\
\hline 9 & HCT \\
\hline 10 & Expower 57 \\
\hline 11 & Expower 57 \\
\hline 12 & Expower 59 \\
\hline 13 & Expower 59 \\
\hline 14 & Expower 59 \\
\hline 15 & Expower 59 \\
\hline 16 & Expower 59 \\
\hline 17 & Expower 59 \\
\hline 18 & Expower 57 \\
\hline 19 & Expower 57 \\
\hline 20 & Expower 57 \\
\hline 21 & Expower 57 \\
\hline Fonte: Autor \\
\hline
\end{tabular}


APÊNDICE L -Relação dos dados obtidos.

Tabela 30 - Dados obtidos.

\begin{tabular}{|c|c|c|c|c|}
\hline Bateria/Medida & $\begin{array}{c}\text { Resistência } \\
\text { interna }\end{array}$ & $\begin{array}{c}\text { Capacitância } \\
\text { interna }\end{array}$ & $\begin{array}{l}\text { Tempo de } \\
\text { descarga }\end{array}$ & $\mathrm{SOH}$ \\
\hline$\frac{1 / 1}{1 / 2}$ & 0,2858 & 0,0558 & $\begin{array}{l}03: 48: 00 \\
032: 463\end{array}$ & $\frac{82,61}{8,07}$ \\
\hline$\frac{1 / 2}{1 / 3}$ & $\frac{0,3154}{0,2864}$ & $\frac{0,0547}{0,0413}$ & $\begin{array}{l}03: 46: 350 \\
0334.50\end{array}$ & $\frac{82,07}{81,52}$ \\
\hline$\frac{1 / 4}{1 / 5}$ & $\frac{0.4247}{0.4227}$ & $\frac{0.0425}{0.0425}$ & $\begin{array}{l}03: 43: 00 \\
033: 42: 00\end{array}$ & $\frac{80,8}{80,43}$ \\
\hline $1 / 6$ & 0,3956 & $0,0,01955$ & 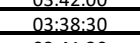 & $\begin{array}{l}\frac{10,45}{79,17} \\
7\end{array}$ \\
\hline$\frac{1 / 7}{1 / 8}$ & $\frac{0,2916}{0,4255}$ & $\frac{0,0243}{0,0205}$ & $\begin{array}{l}03: 41: 30 \\
0.3 \cdot 31.00\end{array}$ & $\begin{array}{r}80,25 \\
80,07\end{array}$ \\
\hline$\frac{1 / 8}{1 / 9}$ & $\frac{0.4255}{0.4707}$ & $\frac{0,0205}{0.02}$ & $\begin{array}{l}\frac{0344: 100}{03: 42: 30} \\
0\end{array}$ & $\begin{array}{l}80,07 \\
80,62\end{array}$ \\
\hline$\frac{1 / 10}{1 / 11}$ & $\frac{0,4383}{0,4777}$ & $\frac{0,0212}{0.0175}$ & $\begin{array}{l}\frac{03388: 30}{033: 38: 30} \\
0.58\end{array}$ & $\frac{79,17}{79,17}$ \\
\hline$\frac{1 / 12}{1 / 12}$ & 0.5362 & 0.0148 & $03: 03: 030$ & $\begin{array}{l}79.89 \\
7980\end{array}$ \\
\hline$\frac{1 / 13}{1 / 14}$ & $\frac{0,1597}{1,0506}$ & $\frac{0,0188}{0,0066}$ & $\begin{array}{l}0353: 5000 \\
03: 26: 30\end{array}$ & $\frac{11,99}{74,82}$ \\
\hline$\frac{1 / 15}{1 / 16}$ & $\frac{1,1066}{1,2451}$ & $\frac{0,005}{0,0049}$ & $\begin{array}{l}03: 2: 2: 30 \\
03: 18: 00\end{array}$ & $\begin{array}{ll}74,09 \\
71,74\end{array}$ \\
\hline $1 / 17$ & 1,1775 & 0,0038 & $03: 19: 00$ & 72,1 \\
\hline$\frac{1118}{1 / 19}$ & $\frac{1,0 / 41}{0,9477}$ & $\frac{0,0069}{0,0077}$ & $\begin{array}{l}03: 27.7 \\
03: 30\end{array}$ & $\frac{75,18}{76,09}$ \\
\hline$\frac{1 / 20}{1 / 21}$ & $\frac{1.1895}{1.5034}$ & $\frac{0,0051}{0.00042}$ & $03: 18: 30$ & $\frac{71.92}{692}$ \\
\hline$\frac{1 / 21}{1 / 22}$ & $\begin{array}{l}1,5354 \\
0.5014\end{array}$ & $\frac{0,0,42}{0,016}$ & $\begin{array}{ll}03: 400 \\
03: 40: 00\end{array}$ & $\frac{16,71}{79,71}$ \\
\hline$\frac{1 / 23}{1 / 24}$ & $\frac{0,7189}{0,9497}$ & $\frac{0,0114}{0,007}$ & $\begin{array}{l}\frac{03343: 30}{032: 30} \\
0.26\end{array}$ & $\begin{array}{ll}77,72 \\
74,82\end{array}$ \\
\hline $1 / 25$ & $0,0,517$ & 0,0162 & $\begin{array}{l}033: 39: 00 \\
03: 39\end{array}$ & 79,35 \\
\hline$\frac{1 / 26}{1 / 27}$ & $\frac{1,472}{10103}$ & $\frac{0,005}{0,007}$ & $\begin{array}{l}0.3: 32: 00 \\
03: 22: 00\end{array}$ & $\frac{69,93}{73,19}$ \\
\hline$\frac{1 / 28}{1128}$ & 1,3243 & 0,004 & 02::0::30 & 43,66 \\
\hline$\frac{1 / 29}{1 / 30}$ & $\frac{1,2761}{0.8908}$ & $\frac{0,0053}{0,0079}$ & $\begin{array}{l}03: 05: 30 \\
03: 15: 30\end{array}$ & $\frac{67,21}{70,83}$ \\
\hline$\frac{1 / 31}{11 / 22}$ & $\frac{0,8251}{18050}$ & 0 & $\begin{array}{ll}03: 30: 00 \\
0.0200\end{array}$ & $\frac{76,09}{5.57}$ \\
\hline $1 / 33$ & $\frac{1.80599}{0.5814}$ & $\frac{0,003}{0.0145}$ & $02: 4: 280$ & $\begin{array}{l}\frac{5.1}{79,17} \\
\end{array}$ \\
\hline$\frac{1 / 144}{1 / 35}$ & $\frac{0,4904}{0.7995}$ & $\frac{0,0181}{0,0083}$ & $\begin{array}{l}03: 37: 00 \\
033: 3: 00\end{array}$ & $\frac{78,62}{77,17}$ \\
\hline$\frac{1 / 36}{1 / 177}$ & 0,6426 & 0,0106 & $\begin{array}{l}03: 38: 30 \\
0.3230\end{array}$ & $\begin{array}{l}79,17 \\
7201\end{array}$ \\
\hline$\frac{1 / 37}{1 / 38}$ & $\frac{0,9535}{0,7788}$ & $\frac{0,0003}{0,0081}$ & $\begin{array}{l}05: 24.00 \\
03: 33: 00\end{array}$ & $\begin{array}{l}\frac{13,91}{77,17} \\
7\end{array}$ \\
\hline$\frac{1 / 39}{1 / 40}$ & $\frac{1,5916}{1,0538}$ & $\frac{0.0019}{0.0065}$ & $\begin{array}{l}02: 24: 00 \\
0225: 00\end{array}$ & $\frac{52,17}{63,41}$ \\
\hline$\frac{1 / 41}{1 / 42}$ & $\frac{1,0911}{1,2799}$ & $\frac{0.0062}{0.0052}$ & $03: 23: 00$ & $\frac{73.55}{70,47}$ \\
\hline$\frac{1 / 43}{1 / 43}$ & 0,9247 & 0,0073 & $\begin{array}{l}03: 32: 00 \\
\end{array}$ & 76,81 \\
\hline$\frac{1 / 44}{1 / 45}$ & $\frac{0.531}{0.036}$ & $\frac{0,0155}{0.074}$ & $\begin{array}{l}03: 43: 30 \\
0.3234 .00\end{array}$ & \\
\hline $1 / 46$ & 1,3959 & 0,0071 & $02: 37: 30$ & 57,07 \\
\hline$\frac{1 / 47}{1 / 48}$ & $\frac{1.1387}{20041}$ & $\frac{0,0057}{0.004}$ & 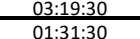 & $\frac{72.28}{33.15}$ \\
\hline $1 / 49$ & & & & \\
\hline $1 / 50$ & 1,2253 & 0,0056 & $\begin{array}{l}01: 47: 00 \\
\end{array}$ & $\begin{array}{l}38,77 \\
\end{array}$ \\
\hline
\end{tabular}

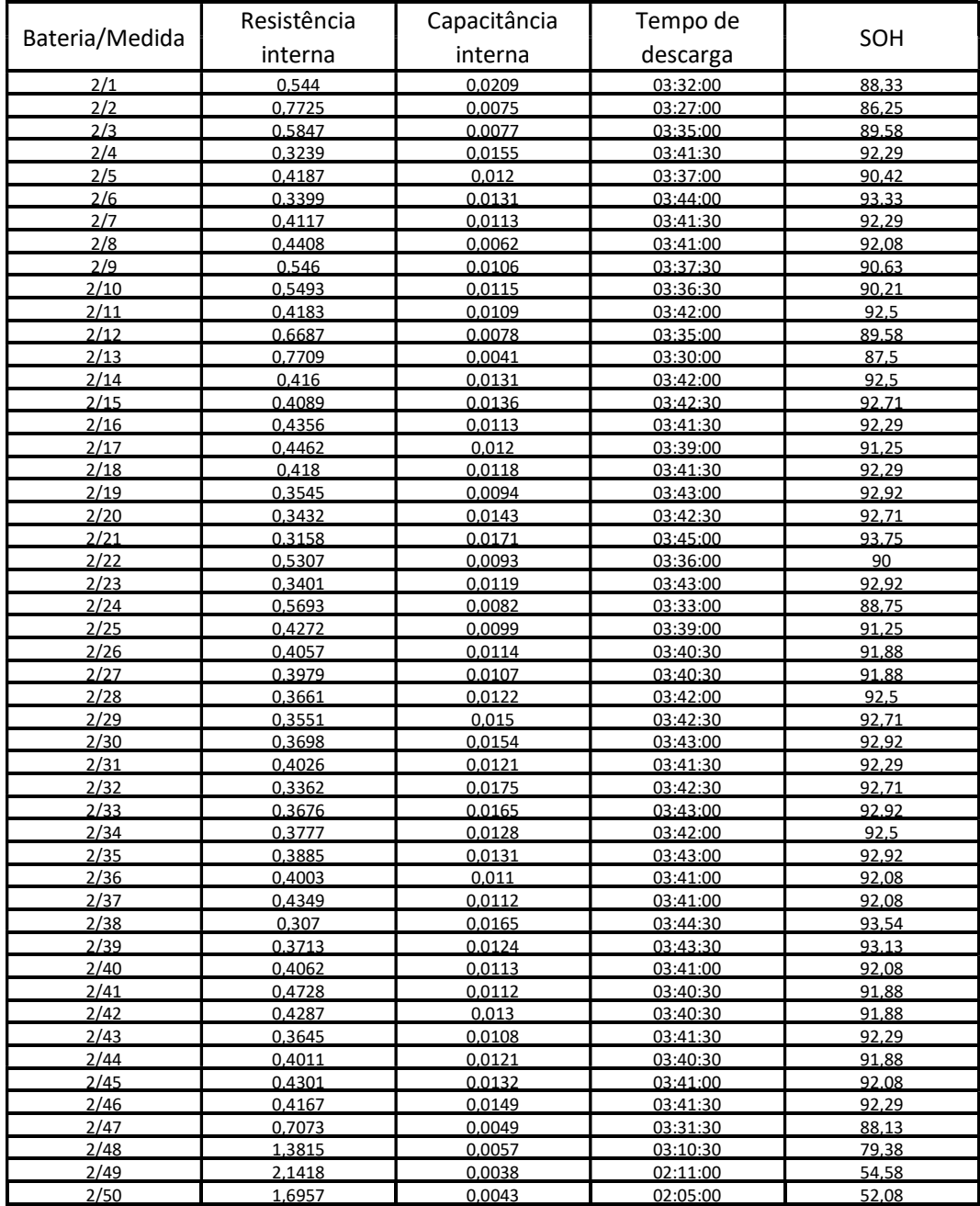



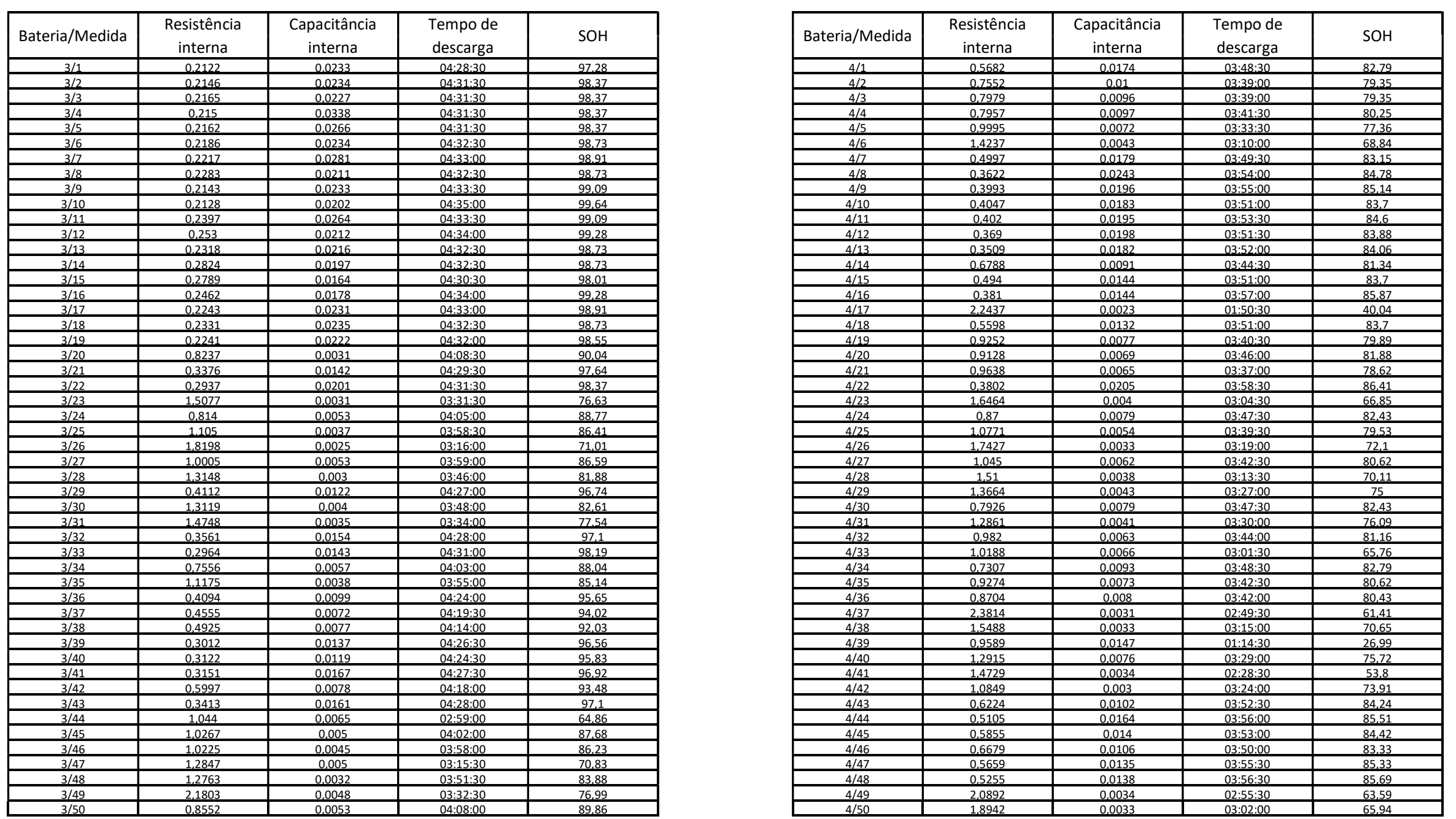

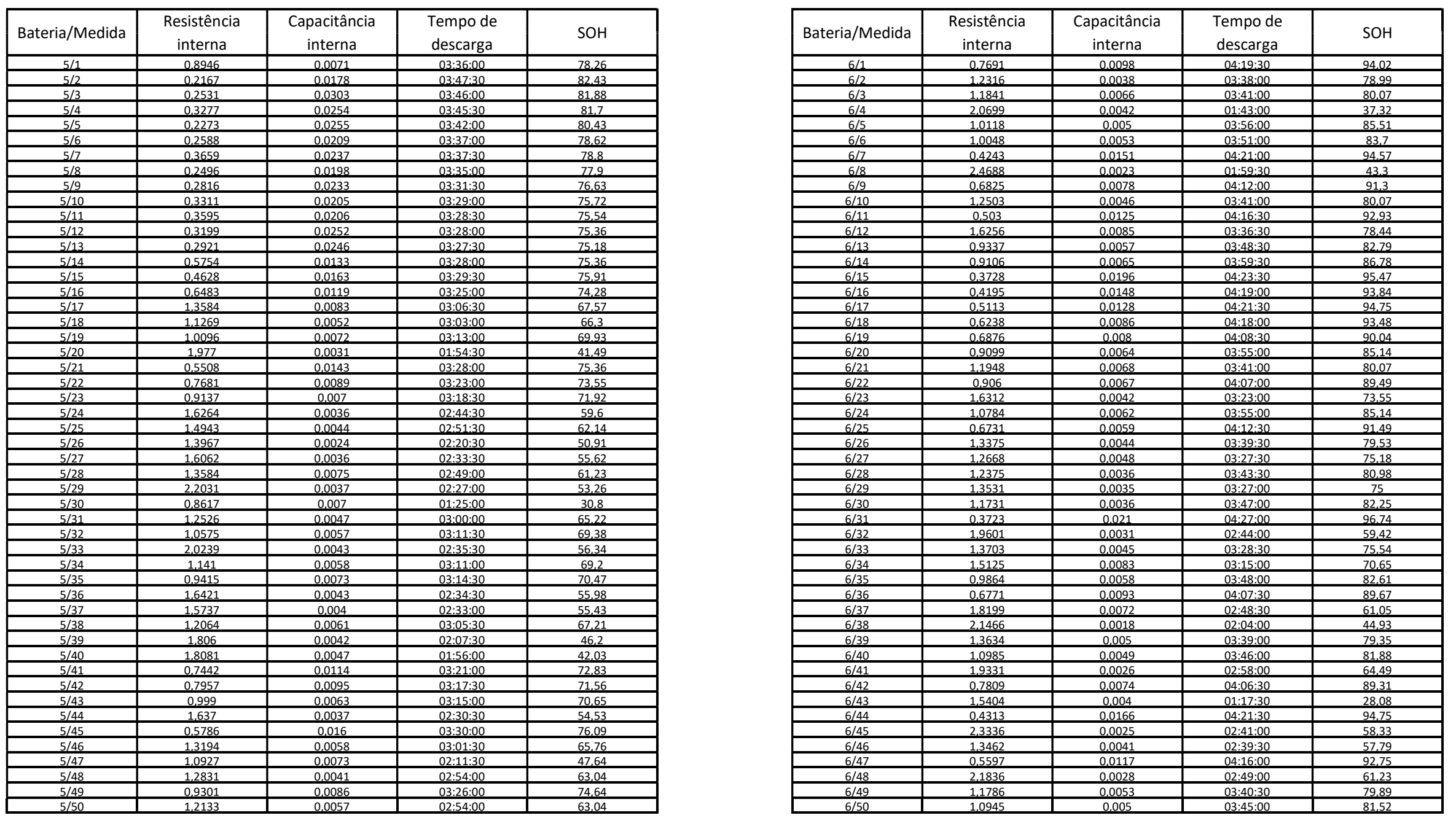

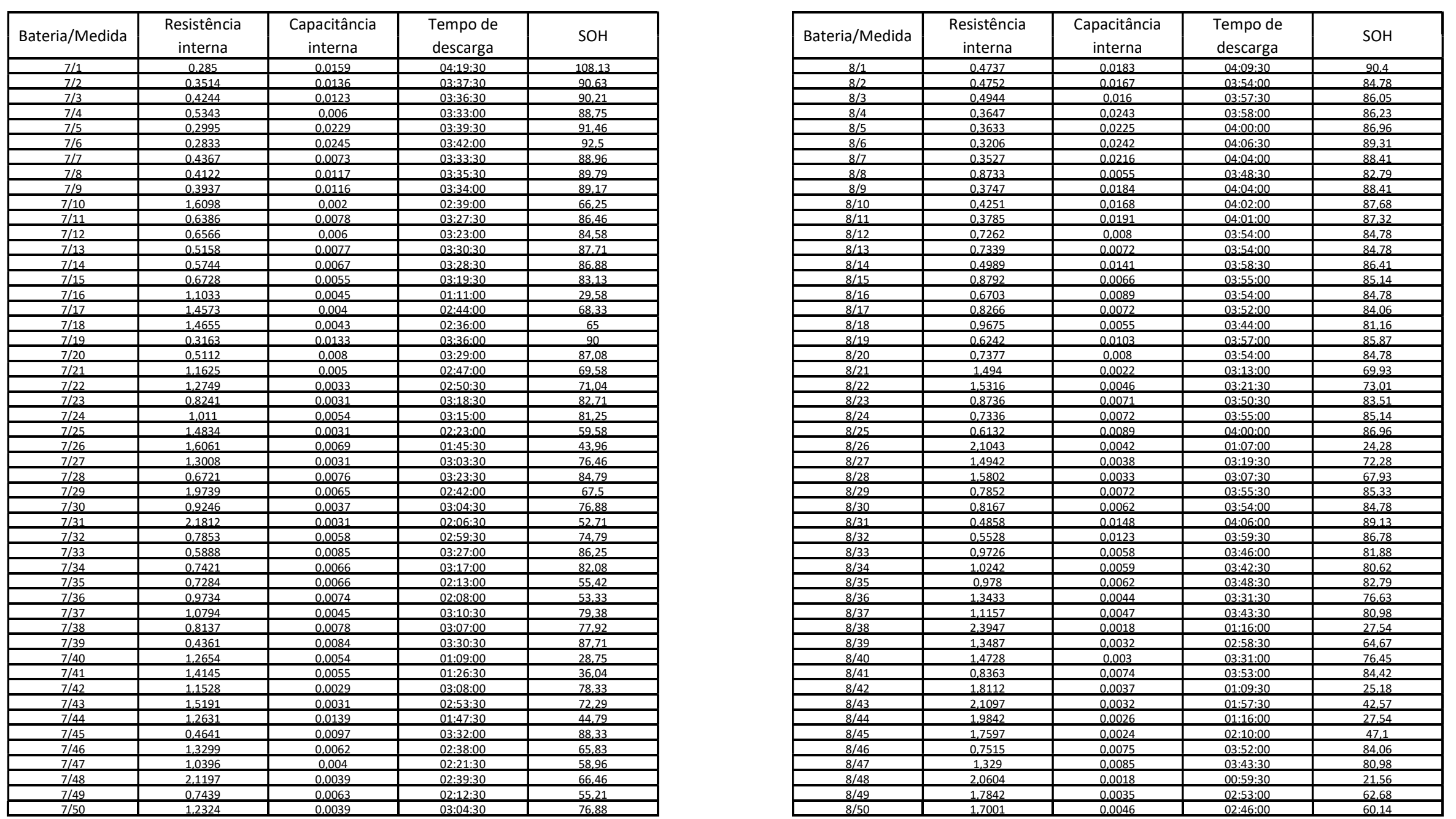


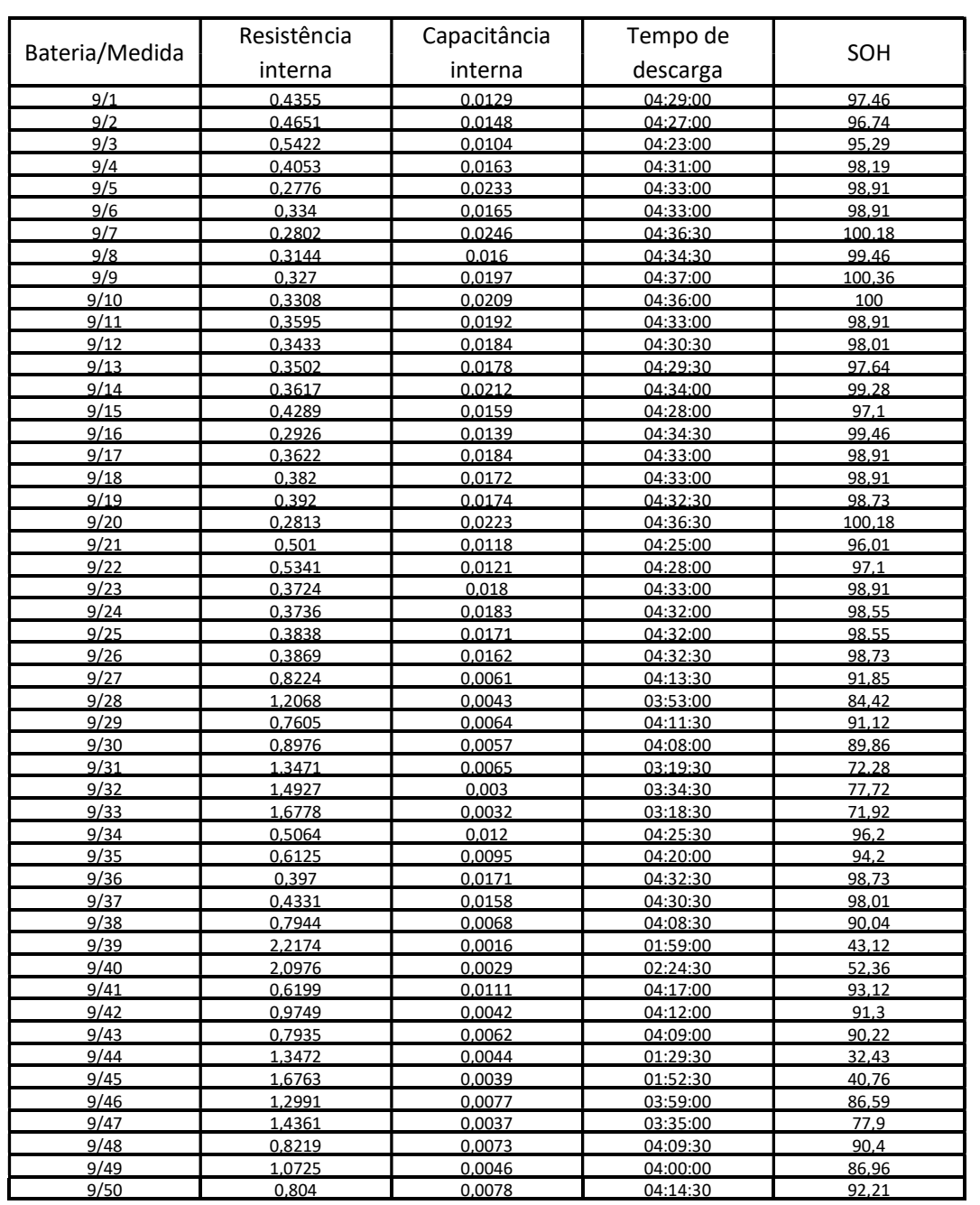

\begin{tabular}{|c|c|c|c|c|}
\hline Bateria/Medida & $\begin{array}{c}\text { Resistência } \\
\text { interna }\end{array}$ & $\begin{array}{l}\text { Capacitância } \\
\text { interna }\end{array}$ & $\begin{array}{l}\text { Tempo de } \\
\text { descarga }\end{array}$ & $\mathrm{SOH}$ \\
\hline $10 / 1$ & 0,3206 & 0,0133 & $03: 40: 00$ & 91,67 \\
\hline$\frac{10 / 2}{10 / 3}$ & $\begin{array}{l}0,3677 \\
0 ., 3855\end{array}$ & $\begin{array}{l}0,0117 \\
0,0116\end{array}$ & $\begin{array}{l}03: 31: 130 \\
033: 100\end{array}$ & $\begin{array}{l}88,13 \\
87,92 \\
\end{array}$ \\
\hline $10 / 4$ & 0,3411 & $\frac{0,0191}{0,0191}$ & 03:32:30 & 88,54 \\
\hline$\frac{10 / 5}{10 / 6}$ & $\frac{0.2881}{0,8742}$ & $\frac{0,0189}{0,0048}$ & $\begin{array}{l}\frac{03: 3: 00}{03: 14: 00} \\
0.330\end{array}$ & $\frac{90}{80,83}$ \\
\hline $10 / 7$ & 0,4342 & $\frac{0,0095}{0,012}$ & $\begin{array}{l}03: 31: 00 \\
03: 3100\end{array}$ & 87,92 \\
\hline$\frac{10 / 8}{10 / 9}$ & 0,4467 & 0,0124 & $\begin{array}{l}03: 30: 30 \\
030.30: 30\end{array}$ & $\frac{87,71}{8771}$ \\
\hline $10 / 10$ & $0,0,3052$ & $\frac{0,0112}{0,0167}$ & 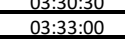 & $\frac{81,71}{88,75}$ \\
\hline$\frac{10 / 11}{10 / 12}$ & $\frac{0,3039}{0,3491}$ & 0,0169 & $\begin{array}{l}03: 34: 30 \\
0.333: 30\end{array}$ & $\begin{array}{r}89,38 \\
88.96\end{array}$ \\
\hline $10 / 13$ & $\begin{array}{l}0.3451 \\
0.0368 \\
0.03\end{array}$ & 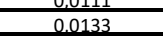 & $\begin{array}{l}\frac{0.535 .30}{03: 33: 00} \\
0.00\end{array}$ & $\frac{80,90}{88,75}$ \\
\hline $10 / 14$ & & 0.0116 & $03: 27: 3$ & 86,46 \\
\hline$\frac{10115}{10 / 16}$ & $\frac{0,443}{0,4469}$ & $\frac{0,0085}{0,0102}$ & $\begin{array}{l}03: 3: 2: 00 \\
0.330: 30\end{array}$ & $\frac{88,54}{87,71}$ \\
\hline $10 / 17$ & $\frac{0,3723}{10771}$ & 0,013 & $03: 32: 30$ & 88,54 \\
\hline$\frac{10118}{10 / 19}$ & $\frac{1,0774}{0,443}$ & $\frac{0,0009}{0,0083}$ & $\begin{array}{l}\frac{033: 10: 00}{03: 34: 30} \\
0.0\end{array}$ & \\
\hline$\frac{10 / 20}{1020}$ & 0.5751 & 0,0065 & $03: 25: 00$ & 85.42 \\
\hline $10 / 21$ & 0,4828 & $\frac{0,0097}{0,0097}$ & 0 & 88,54 \\
\hline$\frac{10 / 22}{1023}$ & 0,9676 & $\frac{0,0048}{0,0071}$ & $\begin{array}{l}02: 53: 00 \\
0.3212: 30\end{array}$ & $\frac{72,08}{80,21}$ \\
\hline $10 / 24$ & 1,0652 & 0,0052 & 03:07:00 & 77,92 \\
\hline$\frac{1025}{10 / 26}$ & $\frac{1,7792}{1,7903}$ & $\frac{0,0024}{0,0022}$ & $\begin{array}{l}02: 22: 00 \\
0.22900\end{array}$ & $\frac{66,67}{62.08}$ \\
\hline $10 / 27$ & 1,0219 & 0,0003 & $\begin{array}{l}02: 27: 00 \\
0.025: 620\end{array}$ & 61,25 \\
\hline & & & & \\
\hline $10 / 30$ & 1,2469 & $\frac{0,0067}{0,0678}$ & $\begin{array}{l}01: 48: 00 \\
01: 48: 00\end{array}$ & 45 \\
\hline$\frac{10 / 31}{10 / 32}$ & $\frac{0.5236}{1.1146}$ & $\frac{0,0076}{0,0053}$ & $\frac{03: 25: 30}{0.304: 30}$ & $\frac{85,63}{76,88}$ \\
\hline $10 / 33$ & 1,3701 & 0,0132 & $02: 53: 30$ & 72,29 \\
\hline$\frac{10 / 34}{10 / 25}$ & $\frac{0.4778}{0.8529}$ & $\frac{0.0098}{0.0046}$ & $\begin{array}{l}\frac{03: 31: 30}{0331300} \\
0.00\end{array}$ & $\frac{88,13}{80.42}$ \\
\hline $10 / 36$ & 1,675 & 0,0052 & $02: 20: 00$ & 58,33 \\
\hline$\frac{10 / 37}{10 / 38}$ & $\frac{0,8408}{1.975}$ & $\frac{0,0046}{0,032}$ & $\begin{array}{l}03: 17: 30 \\
0.20 .5300\end{array}$ & $\frac{82,29}{7708}$ \\
\hline $\begin{array}{l}10 / 39 \\
10 / 10 \\
\end{array}$ & $\begin{array}{l}0,8993 \\
0.0906\end{array}$ & 0,006 & $\begin{array}{l}03: 13: 00 \\
02: 5: 30\end{array}$ & $\frac{80,42}{7470}$ \\
\hline$\frac{10 / 41}{101}$ & 1,9962 & 0,0031 & $01: 15: 30$ & 31.46 \\
\hline$\frac{10 / 42}{10123}$ & $0 ., 3024$ & 0,0154 & $\begin{array}{l}03: 37: 30 \\
02323: 00\end{array}$ & \\
\hline $10 / 44$ & 1,3363 & $\frac{0,014}{0,0184}$ & $\begin{array}{l}\frac{0.53600}{02: 55: 00} \\
0.00\end{array}$ & $\frac{90}{72,92}$ \\
\hline$\frac{10 / 45}{10 / 46}$ & $\frac{2,0925}{1,5828}$ & 0,0062 & $\begin{array}{l}01: 2: 30 \\
0.155: 00 \\
0.00\end{array}$ & $\frac{36,04}{47,92}$ \\
\hline$\frac{10 / 47}{10 / 49}$ & 0,8306 & 0,0055 & $03: 15: 30$ & 81,46 \\
\hline & & & $0<258530$ & \\
\hline & & & 03:10:30 & 79,38 \\
\hline
\end{tabular}



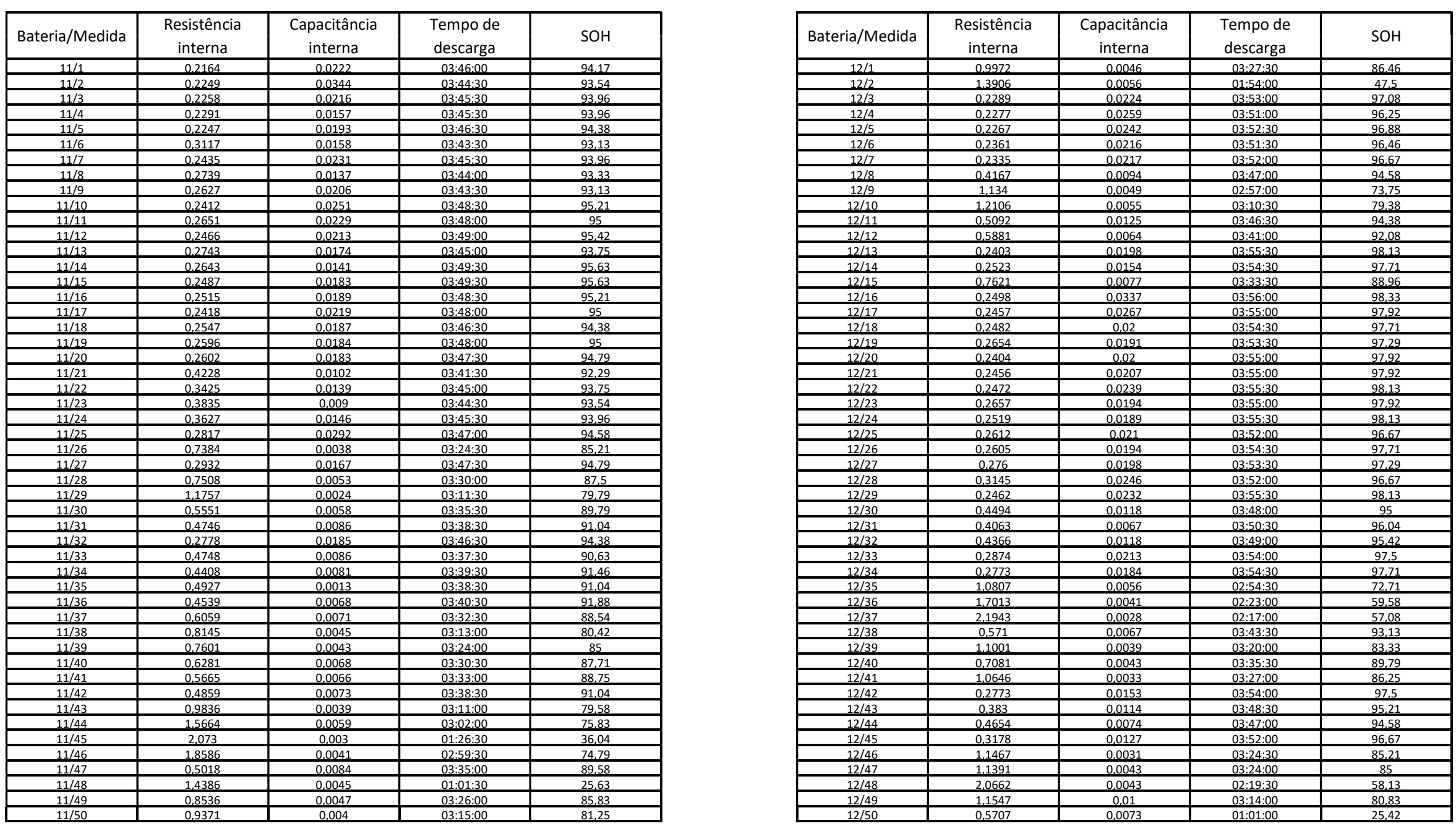

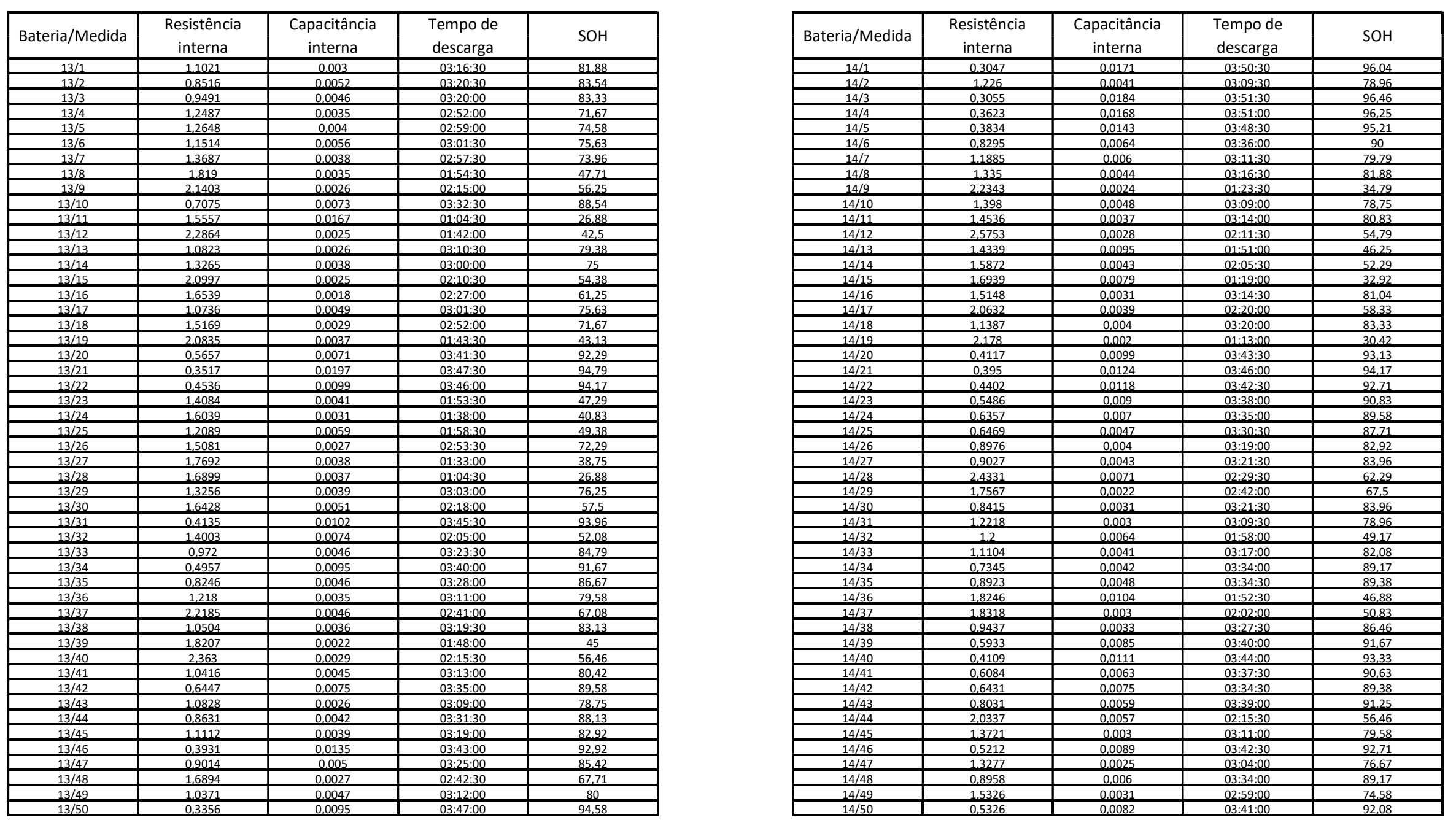


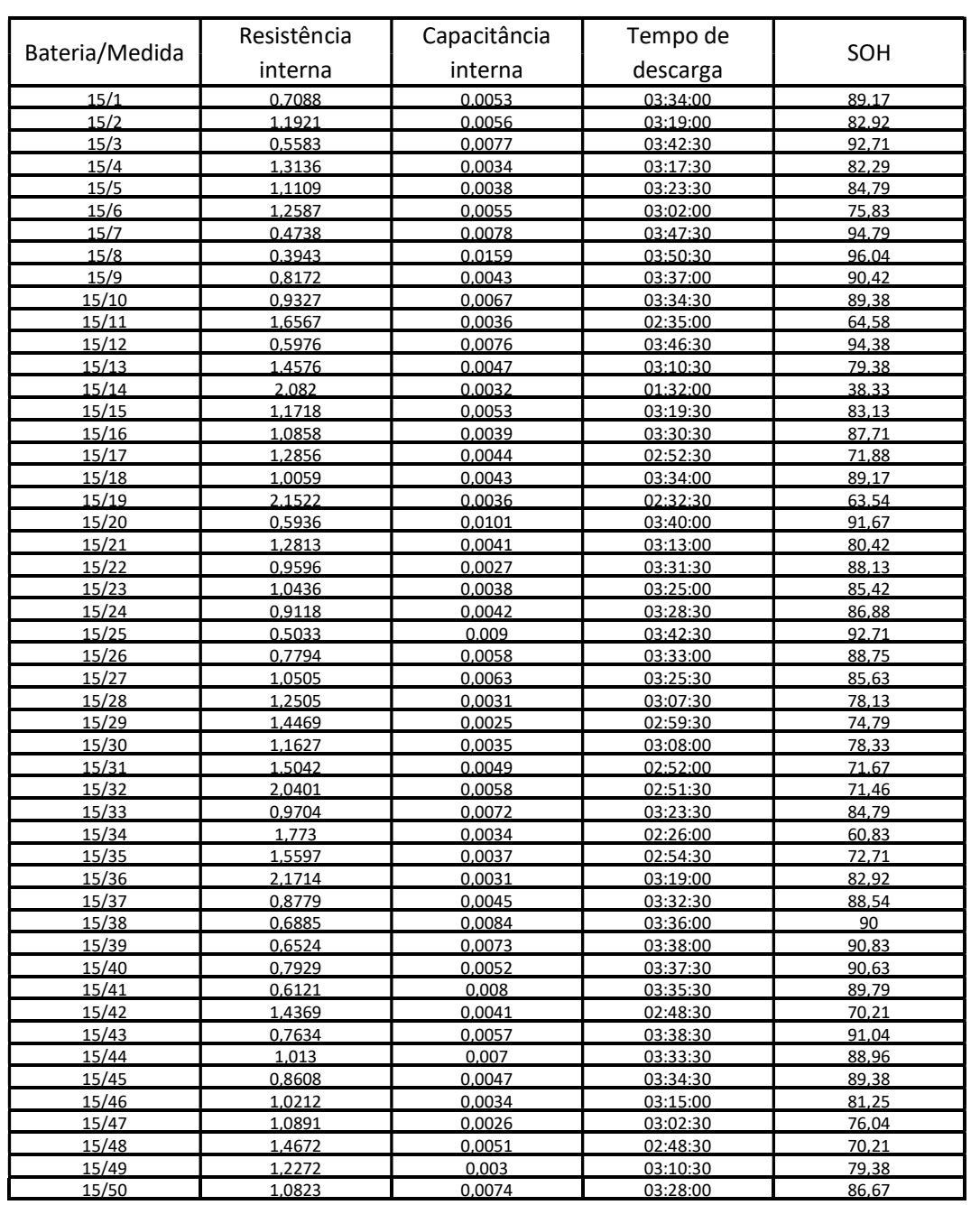

\begin{tabular}{|c|c|c|c|c|}
\hline Bateria/Medida & $\begin{array}{c}\text { Resistência } \\
\text { interna }\end{array}$ & $\begin{array}{l}\text { Capacitância } \\
\text { interna }\end{array}$ & $\begin{array}{l}\text { Tempo de } \\
\text { descarga }\end{array}$ & $\mathrm{SOH}$ \\
\hline $16 / 1$ & 0,2831 & 0,0222 & $\begin{array}{l}03: 51: 30 \\
\end{array}$ & 96,46 \\
\hline $16 / 2$ & 0,3135 & 0,0187 & 03:50:00 & 95,83 \\
\hline $16 / 3$ & 0,306 & 0,0175 & 03:53:00 & 97,08 \\
\hline $16 / 4$ & 0,9744 & 0,0045 & 03:29:00 & 87,08 \\
\hline $16 / 5$ & 1,1614 & 0,0039 & $03: 23: 30$ & 84,79 \\
\hline $16 / 6$ & 0,8071 & 0,0053 & $03: 35: 30$ & 89,79 \\
\hline $16 / 7$ & 2,1233 & 0,0045 & $01: 57: 00$ & 48,75 \\
\hline $16 / 8$ & 0,3873 & 0,0109 & $03: 54: 00$ & 97,5 \\
\hline $16 / 9$ & 1,1667 & 0,0035 & $03: 22: 00$ & 84,17 \\
\hline $16 / 10$ & 1,3195 & 0,0066 & 03:06:00 & 77,5 \\
\hline $16 / 11$ & 1,4666 & 0,007 & $02: 48: 30$ & 70,21 \\
\hline $16 / 12$ & 2,5108 & 0,0025 & $01: 53: 30$ & 47,29 \\
\hline $16 / 13$ & 1,184 & 0,0038 & 03:19:00 & 82,92 \\
\hline $16 / 14$ & 1,751 & 0,0055 & 02:03:30 & 51,46 \\
\hline $16 / 15$ & 0,5427 & 0,0072 & 03:48:00 & 95 \\
\hline $16 / 16$ & 1,4247 & 0,0035 & 03:33:00 & 88,75 \\
\hline $16 / 17$ & 1,6978 & 0,0026 & $01: 32: 30$ & 38,54 \\
\hline $16 / 18$ & 0,8632 & 0,0057 & 03:44:30 & 93,54 \\
\hline$\frac{16 / 19}{16 / 20}$ & 1,6716 & 0,0028 & 03:18:30 & 82.71 \\
\hline$\frac{16 / 20}{16 / 21}$ & $\frac{0,3282}{0.372}$ & 0,0126 & 03:52:30 & $\begin{array}{r}96,88 \\
0,25\end{array}$ \\
\hline$\frac{16 / 21}{16 / 22}$ & 0,372 & 0,0158 & $\begin{array}{l}03: 51: 00 \\
03 \cdot 32 \cdot 3\end{array}$ & $\frac{96,25}{90.19}$ \\
\hline$\frac{16 / 22}{16 / 23}$ & $\frac{0,9393}{1,1179}$ & $\frac{0,0048}{0,0028}$ & $\frac{03: 32: 30}{03: 00}$ & $\frac{88,54}{87,5}$ \\
\hline$\frac{16 / 23}{16 / 24}$ & $\begin{array}{l}\frac{1,11 / 9}{1,9943} \\
\end{array}$ & $\begin{array}{l}0,0028 \\
0,0034\end{array}$ & $\begin{array}{l}\frac{03: 30: 00}{01: 50: 30} \\
\end{array}$ & $\frac{8,5}{46,04}$ \\
\hline $16 / 25$ & 0,8476 & 0,0053 & $03: 35: 30$ & 89,79 \\
\hline $16 / 26$ & 0,8403 & 0,0054 & 03:34:00 & 89,17 \\
\hline $16 / 27$ & 0,7505 & 0,0034 & $03: 38: 00$ & 90,83 \\
\hline $16 / 28$ & 1,0542 & 0,0045 & $03: 24: 30$ & 85,21 \\
\hline $16 / 29$ & 0,9431 & 0,0029 & $03: 20: 00$ & 83,33 \\
\hline $16 / 30$ & 1,2353 & 0,0049 & $03: 18: 00$ & 82,5 \\
\hline $16 / 31$ & 1,6931 & 0,004 & $01: 49: 30$ & 45,63 \\
\hline $16 / 32$ & 1,1105 & 0,004 & $03: 13: 30$ & 80,63 \\
\hline $16 / 33$ & 0,4519 & 0,0118 & 03:54:30 & 97,71 \\
\hline $16 / 34$ & 0,5942 & 0,0072 & $03: 44: 00$ & 93,33 \\
\hline $16 / 35$ & 0,6693 & 0,0051 & $03: 42: 30$ & 92.71 \\
\hline $16 / 36$ & 0,6733 & 0,0067 & 03:46:00 & 94,17 \\
\hline $16 / 37$ & 1,3096 & 0,0047 & 03:02:00 & 75,83 \\
\hline $16 / 38$ & 0,5296 & 0,0081 & 03:47:00 & 94,58 \\
\hline $16 / 39$ & 0,9791 & 0,0042 & 03:32:00 & 88,33 \\
\hline $16 / 40$ & 0,7207 & 0,0062 & $03: 47: 00$ & 94,58 \\
\hline $16 / 41$ & 0,6414 & 0,0065 & 03:44:00 & 93,33 \\
\hline $16 / 42$ & 0,5259 & 0,0071 & 03:51:30 & 96,46 \\
\hline $16 / 43$ & 0,5169 & 0,0091 & 03:45:00 & 93,75 \\
\hline $16 / 44$ & 1,6705 & 0,0018 & $02: 43: 30$ & 68,13 \\
\hline $16 / 45$ & 0,87 & 0,0049 & 03:37:00 & 90,42 \\
\hline $16 / 46$ & 0,9144 & 0,0055 & $03: 31: 00$ & 87,92 \\
\hline $16 / 47$ & 0,836 & 0,0051 & 03:36:30 & 90,21 \\
\hline $16 / 48$ & 1.5714 & 0,0037 & 03:11:30 & 79,79 \\
\hline $16 / 49$ & 1.4781 & 0,0066 & 03:15:00 & 81,25 \\
\hline $16 / 50$ & 0,3403 & 0,0093 & 03:56:30 & 98,54 \\
\hline
\end{tabular}



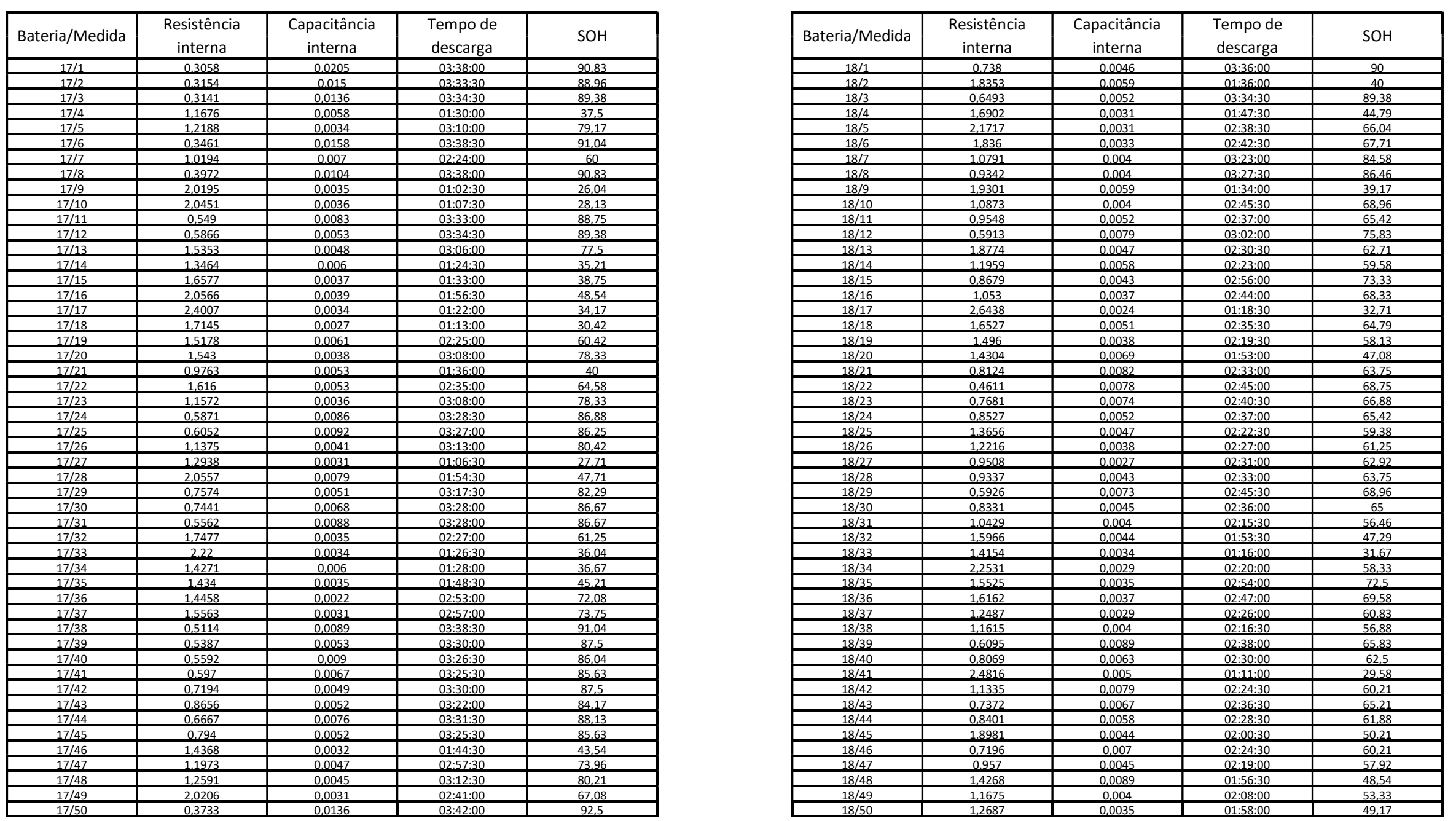


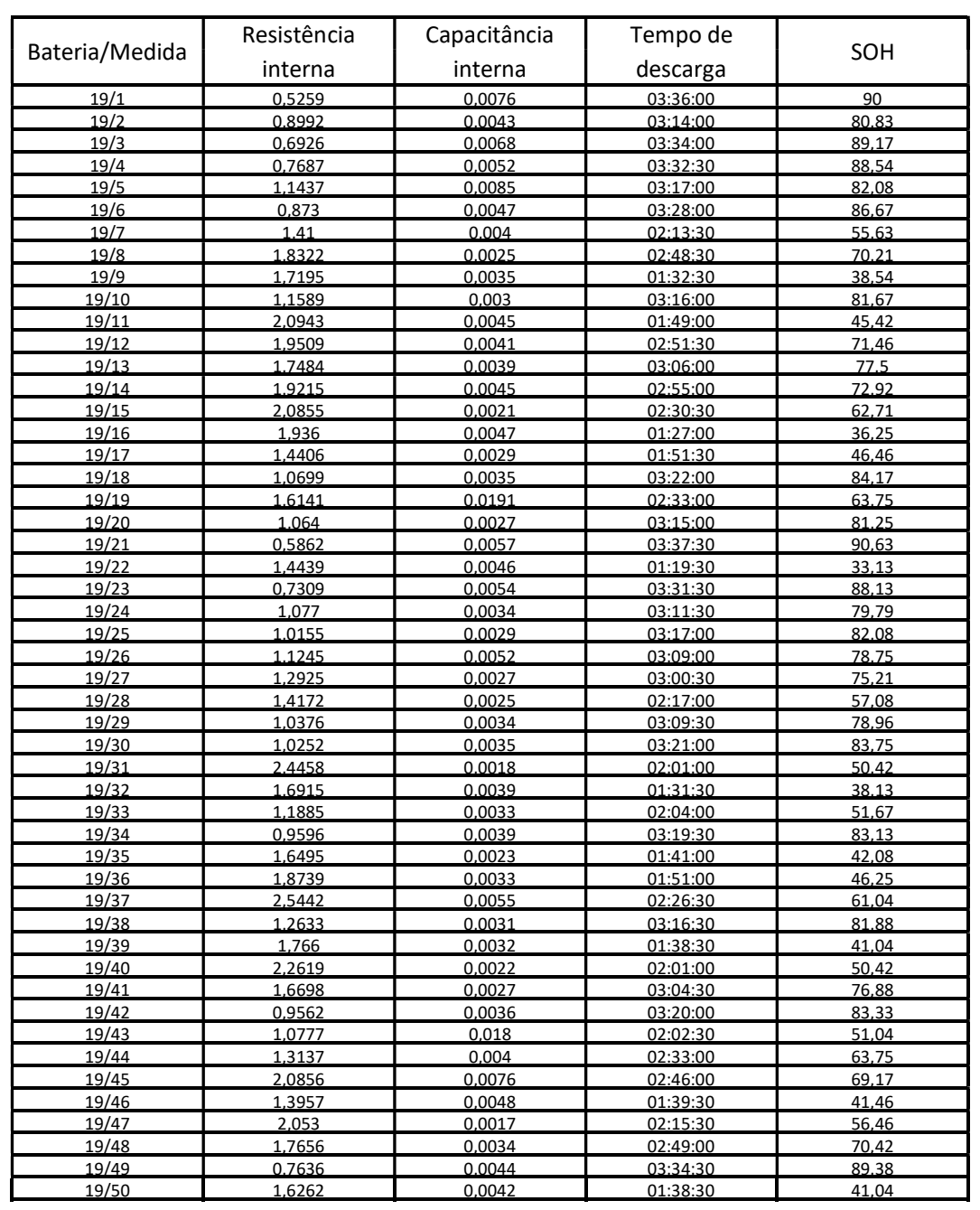

\begin{tabular}{|c|c|c|c|c|}
\hline Bateria/Medida & $\begin{array}{l}\text { Resistência } \\
\text { interna }\end{array}$ & $\begin{array}{c}\text { Capacitância } \\
\text { interna }\end{array}$ & $\begin{array}{l}\text { Tempo de } \\
\text { descarga }\end{array}$ & $\mathrm{SOH}$ \\
\hline $20 / 1$ & 0,3567 & 0,0159 & 03:41:00 & 92,08 \\
\hline $20 / 2$ & 0,8463 & 0,0043 & $03: 12: 30$ & 80,21 \\
\hline $20 / 3$ & 1,2468 & 0,0051 & $03: 08: 30$ & 78,54 \\
\hline $20 / 4$ & 0,9351 & 0,0049 & $03: 23: 30$ & 84,79 \\
\hline $20 / 5$ & 1,103 & 0,0043 & $03: 15: 00$ & 81,25 \\
\hline $20 / 6$ & 1,4528 & 0,0038 & 03:03:00 & 76,25 \\
\hline $20 / 7$ & 1,6975 & 0,0047 & $02: 49: 00$ & 70,42 \\
\hline $20 / 8$ & 1,2005 & 0,0025 & $03: 16: 00$ & 81,67 \\
\hline $20 / 9$ & 1,134 & 0,0036 & $03: 12: 00$ & 80 \\
\hline $20 / 10$ & 1,7746 & 0,003 & $02: 37: 30$ & 65,63 \\
\hline $20 / 11$ & 1,3815 & 0,0081 & $01: 38: 30$ & 41,04 \\
\hline $20 / 12$ & 1,4762 & 0,0037 & $01: 36: 00$ & 40 \\
\hline $20 / 13$ & 0,6121 & 0,0085 & 03:36:00 & 90 \\
\hline $20 / 14$ & 1,6375 & 0,0053 & 03:03:00 & 76,25 \\
\hline $20 / 15$ & 1,1413 & 0,0523 & $03: 18: 00$ & 82,5 \\
\hline $20 / 16$ & 1,8324 & 0,0041 & 03:02:00 & 75,83 \\
\hline $20 / 17$ & 2,0441 & 0,0054 & 02:37:00 & 65,42 \\
\hline $20 / 18$ & 1,2343 & 0,0033 & 03:15:30 & 81,46 \\
\hline $20 / 19$ & 1,8091 & 0,0024 & 01:37:00 & 40,42 \\
\hline$\frac{20 / 20}{20121}$ & $\frac{1,6678}{0.8103}$ & $\frac{0,0054}{0,044}$ & $02: 49: 30$ & $\frac{70,63}{8583}$ \\
\hline$\frac{20 / 21}{20 / 22}$ & 0,7691 & $\begin{array}{l}0,0044 \\
0,0048 \\
\end{array}$ & $\begin{array}{l}3: 60: 00 \\
03: 26: 30 \\
\end{array}$ & $\frac{85,83}{86,04}$ \\
\hline$\frac{201 / 22}{20 / 23}$ & $\frac{0,1051}{1,0531}$ & $\begin{array}{l}0,0048 \\
0,0033\end{array}$ & $\begin{array}{l}03: 6: 30 \\
03: 17: 00 \\
\end{array}$ & $\frac{88,04}{82,08}$ \\
\hline $20 / 24$ & 1,0488 & $\begin{array}{l}0,0033 \\
0,0041\end{array}$ & $\begin{array}{l}03: 16: 30 \\
03: 100 \\
\end{array}$ & $\frac{82,08}{81,88}$ \\
\hline$\frac{20 / 24}{20 / 25}$ & $0,0,6909$ & $\begin{array}{l}0,0041 \\
0,0037\end{array}$ & $\begin{array}{l}03: 16: 30 \\
03: 27: 00 \\
\end{array}$ & $\frac{81,88}{86,25}$ \\
\hline $20 / 26$ & 0,4822 & 0,0066 & $03: 42: 00$ & $\frac{0,25}{92,5}$ \\
\hline$\frac{20120}{20 / 27}$ & $0,0,5458$ & 0,0071 & $\begin{array}{l}03.424 .00 \\
03: 00 \\
\end{array}$ & 90 \\
\hline$\frac{201 / 28}{20 / 28}$ & 0,8454 & 0,0088 & 03:19:00 & 82,92 \\
\hline$\frac{20120}{20 / 29}$ & 0,9721 & 0,0054 & 03:16:00 & 81,67 \\
\hline $20 / 30$ & 1,6403 & 0,0027 & $02: 42: 30$ & 67,71 \\
\hline $20 / 31$ & 0,9412 & 0,0033 & 03:19:00 & 82,92 \\
\hline $20 / 32$ & 1,5656 & 0,0034 & $02: 35: 30$ & 64.79 \\
\hline $20 / 33$ & 1,9241 & 0,007 & $002 \cdot 22 \cdot 30$ & 59,38 \\
\hline $20 / 34$ & 1.5217 & 0,0042 & $0.2 \cdot 9 \cdot 9 \cdot 00$ & 7042 \\
\hline $20 / 35$ & 1,635 & 0,0037 & 0 & $\frac{4.29}{4292}$ \\
\hline $20 / 36$ & 1,2031 & 0,0041 & $02: 26: 30$ & 61,04 \\
\hline $20 / 37$ & 1.5903 & $\frac{10.03}{0.003}$ & 0 & 69.58 \\
\hline $20 / 38$ & 17655 & 0,0051 & $02: 26: 30$ & 61,04 \\
\hline $20 / 39$ & 0.9607 & 0,0035 & 01:24:00 & 85 \\
\hline $20 / 40$ & 1,5981 & 0,0029 & $02: 38: 30$ & 6604 \\
\hline $20 / 41$ & 1,4476 & 0,0028 & 01:34:30 & 39,38 \\
\hline $20 / 42$ & 1,7475 & 0,0049 & $02: 43: 30$ & 68,13 \\
\hline $20 / 43$ & 1,532 & 0,0031 & $01.455: 00$ & 43.75 \\
\hline $20 / 44$ & 1,1752 & 0,0026 & $03: 11: 30$ & 79,79 \\
\hline $20 / 45$ & 1,2874 & 0,0024 & 03:03:30 & 76,46 \\
\hline $20 / 46$ & 0,8201 & 0,0046 & $03: 25: 00$ & 85,42 \\
\hline $20 / 47$ & 1,1302 & 0,0035 & $03: 14: 00$ & 80,83 \\
\hline $20 / 48$ & 1,6312 & 0,0047 & $02: 37: 00$ & 65,42 \\
\hline $20 / 49$ & 0,727 & 0,006 & $03: 32: 30$ & 88,54 \\
\hline $20 / 50$ & 0,6658 & 0,0063 & $03: 35: 30$ & 89,79 \\
\hline
\end{tabular}




\begin{tabular}{|c|c|c|c|c|}
\hline Bateria/Medida & $\begin{array}{c}\text { Resistência } \\
\text { interna }\end{array}$ & $\begin{array}{c}\text { Capacitância } \\
\text { interna }\end{array}$ & $\begin{array}{l}\text { Tempo de } \\
\text { descarga }\end{array}$ & $\mathrm{SOH}$ \\
\hline $21 / 1$ & 1,4626 & 0,0043 & $03: 06: 30$ & 77.71 \\
\hline $21 / 2$ & 0,3528 & 0,0136 & 0 & 92,08 \\
\hline $21 / 3$ & 0,3248 & 0,0158 & $03: 43: 30$ & 93,13 \\
\hline $21 / 4$ & 1,5984 & 0,003 & $03: 13: 30$ & 80,63 \\
\hline $21 / 5$ & 1,6514 & 0,004 & 0 & 69,79 \\
\hline $21 / 6$ & 2,0088 & 0,003 & $02: 02: 00$ & 50,83 \\
\hline $21 / 7$ & 1.8959 & 0,0042 & $01: 17: 30$ & 32.29 \\
\hline $21 / 8$ & 1,3641 & 0,003 & $03: 11: 00$ & 79,58 \\
\hline $21 / 9$ & 0,3824 & 0,0104 & $03: 42: 00$ & 92,5 \\
\hline $21 / 10$ & 0,8491 & 0,0045 & $03: 31: 00$ & 87,92 \\
\hline $21 / 11$ & 0,5728 & 0,0011 & $03: 41: 30$ & 92,29 \\
\hline $21 / 12$ & 1,0911 & 0,0025 & $03: 25: 00$ & 85.42 \\
\hline $21 / 13$ & 1,2027 & 0,0158 & $03: 12: 00$ & 80 \\
\hline $21 / 14$ & 1,5176 & 0,0028 & $02: 27: 00$ & 61,25 \\
\hline $21 / 15$ & 1,6194 & 0,0037 & $02: 21: 30$ & 58,96 \\
\hline $21 / 16$ & 2,3452 & 0,0026 & $02: 42: 00$ & 67,5 \\
\hline $21 / 17$ & 1,7641 & 0,0046 & $01: 46: 30$ & 44,38 \\
\hline $21 / 18$ & 1,0838 & 0,0058 & 03:15:00 & 81,25 \\
\hline $21 / 19$ & 1,6191 & 0,004 & $01: 34: 30$ & 39,38 \\
\hline $21 / 20$ & 1,5538 & 0,0039 & $02: 58: 30$ & 74,38 \\
\hline $21 / 21$ & 1,3434 & 0,0085 & $01: 22: 30$ & 34,38 \\
\hline $21 / 22$ & 1,8133 & 0,0056 & 02:59:00 & 74,58 \\
\hline $21 / 23$ & 2,6967 & 0,003 & $02: 21: 30$ & 58,96 \\
\hline $21 / 24$ & 1,5547 & 0,0059 & 02:51:30 & 71,46 \\
\hline $21 / 25$ & 0,6078 & 0,0069 & $03: 26: 30$ & 86,04 \\
\hline $21 / 26$ & 0,8886 & 0,0046 & 03:18:30 & 82,71 \\
\hline $21 / 27$ & 0,8398 & 0,0044 & 03:25:00 & 85,42 \\
\hline $21 / 28$ & 1,1474 & 0,0022 & 03:06:30 & 77,71 \\
\hline $21 / 29$ & 1,6736 & 0,006 & 03:00:00 & 75 \\
\hline $21 / 30$ & 0,954 & 0,0059 & 03:22:30 & 84,38 \\
\hline $21 / 31$ & 1,0079 & 0,0025 & 03:15:30 & 81,46 \\
\hline $21 / 32$ & 1,3873 & 0,0052 & 02:58:00 & 74,17 \\
\hline $21 / 33$ & 1,3936 & 0,0091 & 01:16:00 & 31,67 \\
\hline $21 / 34$ & 1,2979 & 0,003 & 03:00:30 & 75,21 \\
\hline $21 / 35$ & 1,4504 & 0,003 & 02:20:00 & 58,33 \\
\hline $21 / 36$ & 1,6386 & 0,0038 & 02:31:30 & 63,13 \\
\hline $21 / 37$ & 2,099 & 0,0026 & 03:15:30 & 81,46 \\
\hline $21 / 38$ & 1,2252 & 0,0031 & 03:12:30 & 80,21 \\
\hline $21 / 39$ & 2,1256 & 0,003 & 01:21:00 & 33,75 \\
\hline$\frac{21 / 40}{21 / 14}$ & $\frac{1,0136}{1,21}$ & 0,0032 & 03:22:00 & $\frac{84,17}{78,12}$ \\
\hline$\frac{21 / 41}{21 / 42}$ & $\frac{1,234}{0.9497}$ & $\frac{0,0046}{0,046}$ & $\frac{03: 07: 30}{031 \cdot 39}$ & $\frac{78,13}{83.13}$ \\
\hline$\frac{21 / 42}{21 / 43}$ & $\begin{array}{l}\frac{0,9497}{1,9032} \\
\end{array}$ & $\begin{array}{l}0,0046 \\
0,0023\end{array}$ & $\begin{array}{l}0: 1: 14: 30 \\
01: 14: 00\end{array}$ & $\frac{83,13}{30,83}$ \\
\hline $21 / 44$ & 0,8812 & 0,0046 & 03:30:00 & 87.5 \\
\hline $21 / 45$ & 0,6761 & 0,0059 & $03: 28: 30$ & 86,88 \\
\hline $21 / 46$ & 0,903 & 0,0051 & 03:26:00 & 85,83 \\
\hline $21 / 47$ & 0,9795 & 0,0042 & 03:25:00 & 85,42 \\
\hline 21/48 & 1,2161 & 0,0061 & 01:56:00 & 48,33 \\
\hline $21 / 49$ & 0,4123 & 0,0123 & $03: 47: 30$ & 94,79 \\
\hline $21 / 50$ & 0,3617 & 0,0109 & $03: 43: 30$ & 93,13 \\
\hline
\end{tabular}


ANEXOS

ANEXO A - Extratos do datasheet da bateria Expower (PN: 483757) de 1000mAH

\section{Specification}

\begin{tabular}{|c|c|c|c|c|}
\hline NO. & \multicolumn{2}{|c|}{ Item } & \multicolumn{2}{|r|}{ Specification } \\
\hline 4.1 & \multicolumn{2}{|c|}{ Min capacity } & \multicolumn{2}{|c|}{$1000 \mathrm{mAh} \quad 0.2 \mathrm{C}$ Discharge } \\
\hline 4.2 & \multirow{2}{*}{\multicolumn{2}{|c|}{$\begin{array}{c}\text { Initial Impedance } \\
\text { Weight }\end{array}$}} & \multirow{2}{*}{\multicolumn{2}{|c|}{$\begin{array}{c}\leq 180 \mathrm{~m} \Omega \\
\text { Approx: } 12 \mathrm{~g}\end{array}$}} \\
\hline 4.3 & & & & \\
\hline 4.4 & \multicolumn{2}{|c|}{$\begin{array}{l}\text { Nominal voltage } \\
\text { Fully charge voltage(FC) } \\
\text { Fully discharge voltage(FD) }\end{array}$} & \multicolumn{2}{|r|}{$\begin{array}{l}3.7 \mathrm{~V} \\
4.2 \mathrm{~V} \\
3.0 \mathrm{~V}\end{array}$} \\
\hline 4.5 & \multicolumn{2}{|c|}{ Standard charge current } & \multicolumn{2}{|r|}{$0.5 \mathrm{C}$} \\
\hline 4.6 & \multicolumn{2}{|c|}{ Standard charging method } & \multicolumn{2}{|c|}{$\begin{array}{c}0.5 \mathrm{C} C \mathrm{C} \text { (constant current) charge to } \mathrm{FC} \text {, } \\
\text { then } \mathrm{CV} \text { (constant voltage } \mathrm{FC}) \text { charge till charge } \\
\text { current decline to } \leq 0.01 \mathrm{C}\end{array}$} \\
\hline 4.7 & \multicolumn{2}{|c|}{ Charging time } & \multicolumn{2}{|r|}{ Standard Charging } \\
\hline 4.8 & \multicolumn{2}{|c|}{ Max. charge current } & \multicolumn{2}{|c|}{$\begin{array}{c}\text { Constant Current 1C Constant Voltage FC } 0.01 \mathrm{C} \\
\text { cut-off }\end{array}$} \\
\hline 4.9 & \multicolumn{2}{|c|}{ Max. discharge current } & \multicolumn{2}{|c|}{ Constant current $1 \mathrm{C}$ end voltage FD } \\
\hline 4.10 & \multicolumn{2}{|c|}{ Standard Discharge Current } & \multicolumn{2}{|c|}{ Constant current $0.2 \mathrm{C}$ end voltage FD } \\
\hline 4.11 & \multicolumn{2}{|c|}{ Charge cut-off voltage } & \multicolumn{2}{|r|}{ Ref. 8.1 VDET1 } \\
\hline 4.12 & \multicolumn{2}{|c|}{ Discharge aut-off Voltage } & \multicolumn{2}{|r|}{ Ref. 8.1 VDET2 } \\
\hline 4.13 & $\begin{array}{c}\text { Storage } \\
\text { temperature }\end{array}$ & $\begin{array}{l}-20^{\circ} \mathrm{C}-60^{\circ} \mathrm{C} \\
-20^{\circ} \mathrm{C}-45^{\circ} \mathrm{C} \\
-20^{\circ} \mathrm{C}-28^{\circ} \mathrm{C}\end{array}$ & $\begin{array}{l}\leq 1 \text { month } \\
\leq 3 \text { month } \\
\leq 1 \text { vear }\end{array}$ & $\begin{array}{l}\text { Percentage of recoverable } \\
\text { capacity no less than } 80 \% \text { of the } \\
\text { initial capacities }\end{array}$ \\
\hline 4.14 & \multicolumn{2}{|c|}{ Recoverable capacity } & \multicolumn{2}{|c|}{$\begin{array}{l}\text { Constant current } 0.5 \mathrm{C} \text { charge to } \mathrm{FC} \text {, then } \\
\text { constant voltage } \mathrm{FC} \text { charge to current declines } \\
\text { to } 0.01 \mathrm{C} \text {, rest for } 10 \mathrm{~min} \text {, constant current } 0.5 \mathrm{C} \\
\text { discharge to FD, rest for } 10 \text { min. Repeat above } \\
\text { steps } 3 \text { times, recording the maximum capacity }\end{array}$} \\
\hline 4.15 & \multicolumn{2}{|c|}{ Storage Humidity } & \multicolumn{2}{|r|}{$\leq 75 \% \mathrm{RH}$} \\
\hline 4.16 & \multicolumn{2}{|c|}{ Appearance } & \multicolumn{2}{|c|}{ Without distortion and leakage } \\
\hline 4.17 & \multicolumn{2}{|c|}{ Standard testing condition } & \multicolumn{2}{|c|}{$\begin{array}{l}\text { Temperature: } 23 \pm 5^{\circ} \mathrm{C} \\
\text { Humidity : } \leq 75 \% \mathrm{RH}\end{array}$} \\
\hline
\end{tabular}




\section{Protection circuit}

PCM Standard

\begin{tabular}{|c|c|c|c|c|c|}
\hline Symbol & Name & MIN. & Typical. & MAX. & Unit \\
\hline VDET1 & Over-Charge detect voltage & 4.25 & 4.28 & 4.31 & $\mathrm{~V}$ \\
\hline VDET2 & Over-discharge detect voltage & 2.9 & 3.0 & 3.1 & V \\
\hline IEC & Excess Current threshold & 2 & & 6 & A \\
\hline IDD & Supply aurrent & - & -- & 7 & $\mu \mathrm{A}$ \\
\hline $\mathrm{RD}$ & $\begin{array}{l}\text { Internal resistance in nomal } \\
\text { operation }\end{array}$ & - & -- & 65 & $\mathrm{~m} \Omega$ \\
\hline RWW & \multicolumn{4}{|c|}{$\begin{array}{l}\text { Reverse withstand voltage: reverse voltage adding to pack caused by } \\
\text { load circuit: Max.12 }\end{array}$} & $\mathrm{V}$ \\
\hline RWI & \multicolumn{4}{|c|}{ Reverse current adding to pack caused by load circuit: Max.5 } & A \\
\hline
\end{tabular}

Schematic diagram

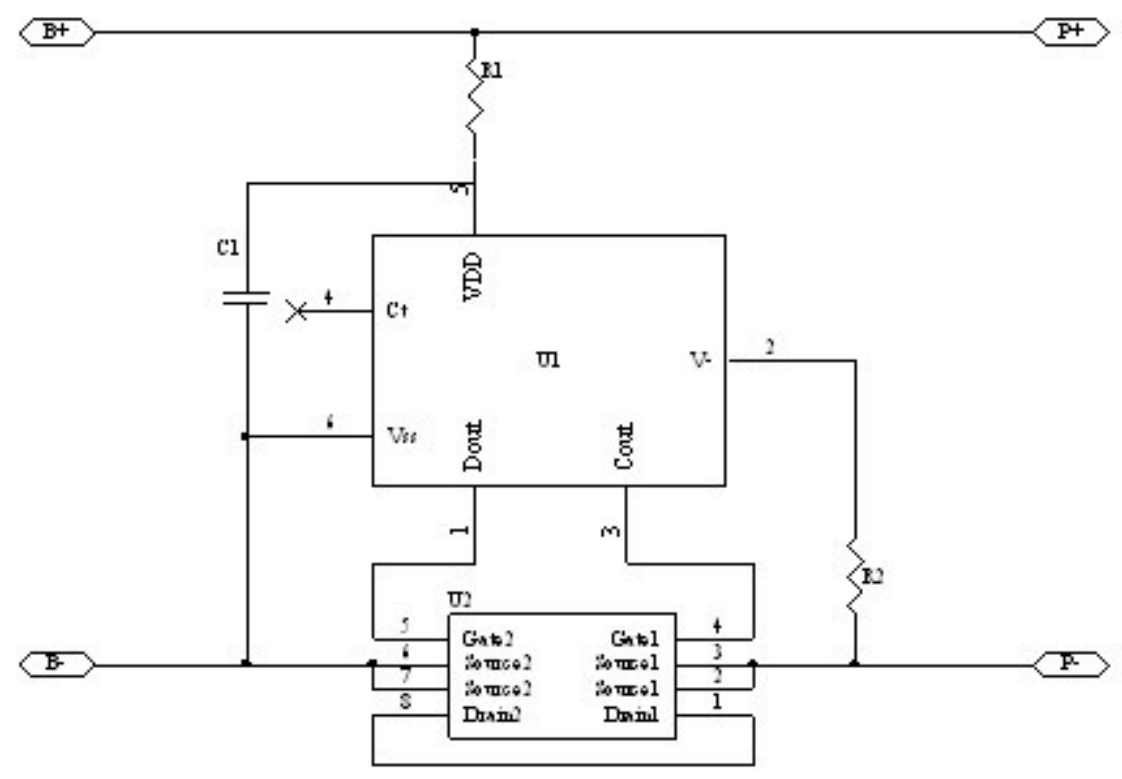




\section{ANEXO B - Extratos do datasheet da bateria Expower (PN: 483759) de 1000mAH \\ SPECIFICATION}

\begin{tabular}{|c|c|c|c|}
\hline No. & Item & Characteristics & Remarks \\
\hline 3.1 & Nominal Capacity & $\begin{array}{l}\text { Minimum:1000mAh } \\
\text { Typical:1000mAh }\end{array}$ & $\begin{array}{c}\text { Fully charged @1C to } 4.2 \mathrm{~V} \\
\text { for } 2.5 \mathrm{hrs} \text {, then discharge to } \\
3.0 \mathrm{~V} @ 0.2 \mathrm{C} .\end{array}$ \\
\hline 3.2 & Nominal Voltage & $3.7 \mathrm{~V}$ & \\
\hline 3.3 & Charging Cut-off Voltage & $4.2 \mathrm{~V}$ & \\
\hline 3.4 & Discharge Cut-off Voltage & $3.0 \mathrm{~V}$ & \\
\hline 3.5 & Maximum Constant & $1000 \mathrm{~mA} \quad 1.0 \mathrm{C}$ & \\
\hline 3.6 & Maximum Continuous & $2000 \mathrm{~mA} \quad 2.0 \mathrm{C}$ & \\
\hline 3.7 & Operating Temperature & $\begin{array}{lr}\text { Charge } & 0 \sim 45^{\circ} \mathrm{C} \\
\text { Discharge } & -20 \quad 60^{\circ} \mathrm{C}\end{array}$ & \\
\hline 3.8 & Storage Temperature & $\begin{array}{l}-2045^{\circ} \mathrm{C} \text { for } 1 \text { Month } \\
-2035^{\circ} \mathrm{C} \text { for } 6 \text { Months }\end{array}$ & \\
\hline 3.9 & Weight & $19.3 \mathrm{~g}$ & Approximate value \\
\hline 3.10 & Storage Voltage & $3.80-3.90 \mathrm{~V}$ & \\
\hline 3.11 & Environmental request & RoHS & $\begin{array}{l}\text { If the materials of the product } \\
\text { and packaging accord with } \\
\text { RoHS standard there will be a } \\
\text { RoHS Id on the box. }\end{array}$ \\
\hline
\end{tabular}

\section{Required Protective Functions}

To ensure safety, the cells need to be assembled with PTC and protective circuitry to prevent abusive situations occur such as over charge and over discharge or over current. The charger and protective circuitry should be consistent with the requirements listed below:

\begin{tabular}{|c|c|c|c|}
\hline No & Device & Items & Requirements \\
\hline 1 & Charger & Charge termination voltage & $4.200 \pm 0.049 \mathrm{~V}$ \\
\hline 2 & \multirow{7}{*}{$\begin{array}{l}\text { Protective Circuitry } \\
\text { (For reference only) }\end{array}$} & Overcharge detection voltage & $4.275 \pm 0.025 \mathrm{~V}$ \\
\hline 3 & & Overcharge release voltage & $4.175 \pm 0.050 \mathrm{~V}$ \\
\hline 4 & & Discharge termination voltage & $3.00 \pm 0.10 \mathrm{~V}$ \\
\hline 5 & & Over discharge detection voltage & $3.0 \pm 0.08 \mathrm{~V}$ \\
\hline 6 & & Over discharge release voltage & $3.0 \pm 0.10 \mathrm{~V}$ \\
\hline 7 & & Over discharge detection value & $2.7 \pm 0.2 \mathrm{~A}$ \\
\hline 8 & & NTC & $10 \pm 5 \% \mathrm{~K}$ \\
\hline
\end{tabular}


ANEXO C - Extratos do datasheet da bateria HCT (PN:

HCT053759P) de 1150mAH

\begin{tabular}{|c|c|c|c|}
\hline \multicolumn{4}{|c|}{ electrical characteristics } \\
\hline No. & ITEM & SPECIFICATION & REMARK \\
\hline 1 & Cell & $053759 \mathrm{P} * 1$ & Li-polymer \\
\hline 2 & Rated Capacity & $1150 \mathrm{mAh}$ & \\
\hline 3 & Internal resistance & $<190 \mathrm{~m} \Omega$ & \\
\hline 4 & Cycle Life & $>300$ & \\
\hline 5 & Nominal Voltage & $3.7 \mathrm{~V} \sim 3.9 \mathrm{~V}$ & \\
\hline 6 & Max. Charge Voltage & $4.2 \mathrm{~V}$ & \\
\hline 7 & $\begin{array}{l}\text { Discharge Cut-off } \\
\text { Voltage }\end{array}$ & $2.75 \mathrm{~V}$ & \\
\hline 8 & $\begin{array}{l}\text { Over-charge } \\
\text { Protection }\end{array}$ & $4.28 \mathrm{~V} \pm 0.025 \mathrm{~V}$ & \\
\hline 9 & $\begin{array}{l}\text { Over-discharge } \\
\text { Protection }\end{array}$ & $3.0 \mathrm{~V} \pm 0.05 \mathrm{~V}$ & \\
\hline 10 & Supply current & $<6 \mathrm{uA}$ & \\
\hline \multirow{2}{*}{13} & \multirow{2}{*}{$\begin{array}{l}\text { Operating } \\
\text { temperature }\end{array}$} & Charging Temperature: $\quad 0 \sim+45^{\circ} \mathrm{C}$ & \\
\hline & & Discharging Temperature: $-20 \sim+60^{\circ} \mathrm{C}$ & \\
\hline \multirow{2}{*}{14} & \multirow{2}{*}{\begin{tabular}{|l} 
Storage \\
temperature
\end{tabular}} & 3 month : $-20 \sim 45^{\circ} \mathrm{C}$ & \\
\hline & & 1 year: $-20 \sim 25^{\circ} \mathrm{C}$ & \\
\hline
\end{tabular}


Pin $1=$ GND

Pin 2 = Resistence 10k (Thermical protection)

Pin $3=$ VCC

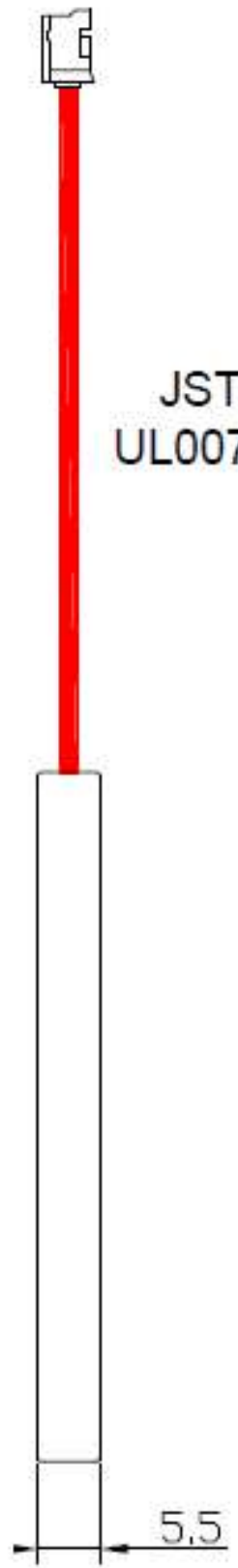

ST-PHR-3

UL007 24 AWG

น)

תㅐㅐㄷㅐ

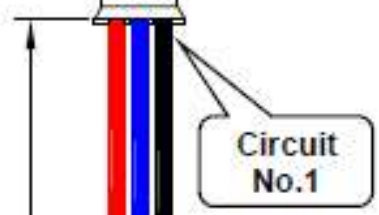

잉

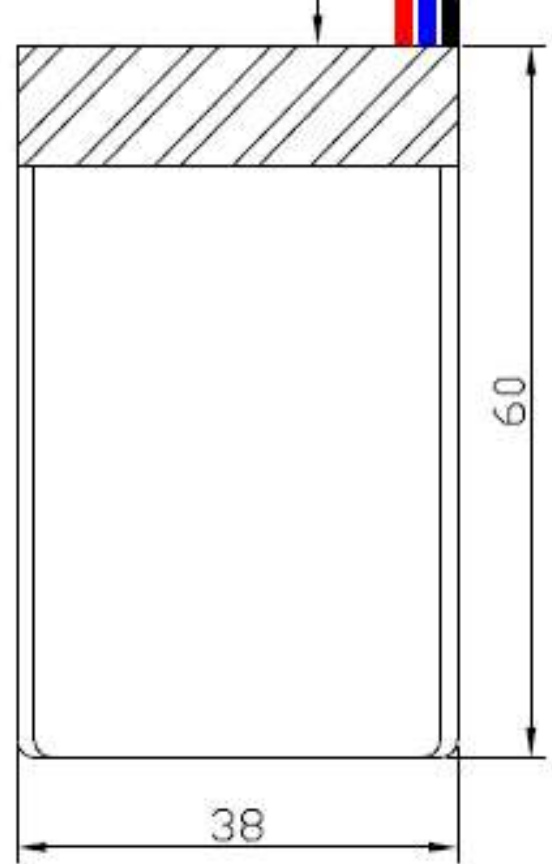

Unit: $\mathrm{mm}$ 


\section{ANEXO D - Extratos do datasheet do LPC1778}

$>$ System:

- ARM Cortex-M3 processor, running at frequencies of up to $120 \mathrm{MHz}$. A Memory Protection Unit (MPU) supporting eight regions is included.

- ARM Cortex-M3 built-in Nested Vectored Interrupt Controller (NVIC).

- Multilayer AHB matrix interconnect provides a separate bus for each AHB master. AHB masters include the CPU, and General Purpose DMA controller. This interconnect provides communication with no arbitration delays unless two masters attempt to access the same slave at the same time.

- Split APB bus allows for higher throughput with fewer stalls between the CPU and DMA. A single level of write buffering allows the CPU to continue without waiting for completion of APB writes if the APB was not already busy.

- Cortex-M3 system tick timer, including an external clock input option.

- Standard JTAG test/debug interface as well as Serial Wire Debug and Serial $>$ WireTrace Port options.

- Emulation trace module supports real-time trace.

- Boundary scan for simplified board testing.

- Non-maskable Interrupt (NMI) input.

> Memory:

- $512 \mathrm{kB}$ on-chip flash program memory with In-System Programming (ISP) and In-Application Programming (IAP) capabilities. The combination of an enhanced flash memory accelerator and location of the flash memory on the CPU local code/data bus provides high code performance from flash.

- 96 kB on-chip SRAM includes:

- $64 \mathrm{kB}$ of SRAM on the CPU with local code/data bus for high-performance CPU access. Two $16 \mathrm{kB}$ SRAM blocks with separate access paths for higher throughput. These SRAM blocks may be used for DMA memory as well as for general purpose instruction and data storage.

- 4032 byte on-chip EEPROM.

> LCD controller, supporting both Super-Twisted Nematic (STN) and Thin-Film Transistors (TFT) displays.

- Dedicated DMA controller. 
- Selectable display resolution (up to $1024 \cdot 768$ pixels).

- Supports up to 24-bit true-color mode.

> External Memory Controller (EMC) provides support for asynchronous static memory devices such as RAM, ROM and flash, as well as dynamic memories such as single data rate SDRAM.

> Eight channel General Purpose DMA controller (GPDMA) on the AHB multilayer matrix that can be used with the SSP, I2S, UART, CRC engine, Analog-toDigital and Digital-to-Analog converter peripherals, timer match signals, GPIO, and for memory-to-memory transfers.

$>$ Serial interfaces:

- Ethernet MAC with MII/RMII interface and associated DMA controller. These functions reside on an independent AHB.

- USB 2.0 full-speed dual port device/host/OTG controller with on-chip PHY and associated DMA controller.

- Five UARTs with fractional baud rate generation, internal FIFO, DMA support, and RS-485/EIA-485 support. One UART (UART1) has full modem control I/O, and one UART (USART4) supports IrDA, synchronous mode, and a smart card mode conforming to ISO7816-3.

- Three SSP controllers with FIFO and multi-protocol capabilities. The SSP interfaces can be used with the GPDMA controller.

- Three enhanced ${ }_{1} \mathrm{C}$-bus interfaces, one with a true open-drain output supporting the full $\mathrm{I}_{2} \mathrm{C}$-bus specification and Fast-mode Plus with data rates of $1 \mathrm{Mbit} / \mathrm{s}$, two with standard port pins. Enhancements include multiple address recognition and monitor mode.

- $\quad I_{2} S$ (Inter-IC Sound) interface for digital audio input or output. It can be used with the GPDMA.

- CAN controller with two channels.

$>$ Digital peripherals:

- SD/MMC memory card interface.

- Up to 165 General Purpose I/O (GPIO) pins depending on the packaging, with configurable pull-up/down resistors, open-drain mode, and repeater mode. All GPIOs are located on an AHB bus for fast access and support Cortex-M3 bit-banding. GPIOs can be accessed by the General Purpose 
DMA Controller. Any pin of ports 0 and 2 can be used to generate an interrupt.

- Two external interrupt inputs configurable as edge/level sensitive. All pins on port 0 and port 2 can be used as edge sensitive interrupt sources.

- Four general purpose timers/counters, with a total of eight capture inputs and ten compare outputs. Each timer block has an external count input. Specific timer events can be selected to generate DMA requests.

- Quadrature encoder interface that can monitor one external quadrature encoder.

- Two standard PWM/timer blocks with external count input option.

- One motor control PWM with support for three-phase motor control.

- Real-Time Clock (RTC) with a separate power domain. The RTC is clocked by a dedicated RTC oscillator. The RTC block includes 20 bytes of batterypowered backup registers, allowing system status to be stored when the rest of the chip is powered off. Battery power can be supplied from a standard 3 $\mathrm{V}$ lithium button cell.

- The RTC will continue working when the battery voltage drops to as low as $2.1 \mathrm{~V}$.

- An RTC interrupt can wake up the CPU from any reduced power mode.

- Event Recorder that can capture the clock value when an event occurs on any of three inputs. The event identification and the time it occurred are stored in registers. The Event Recorder is located in the RTC power domain and can therefore operate as long as there is RTC power.

- Windowed Watchdog Timer (WWDT). Windowed operation, dedicated internal oscillator, watchdog warning interrupt, and safety features.

- CRC Engine block can calculate a CRC on supplied data using one of three standard polynomials. The CRC engine can be used in conjunction with the DMA controller to generate a CRC without CPU involvement in the data transfer.

Analog peripherals:

- 12-bit Analog-to-Digital Converter (ADC) with input multiplexing among eight pins, conversion rates up to $400 \mathrm{kHz}$, and multiple result registers. The 12bit ADC can be used with the GPDMA controller. 
- 10-bit Digital-to-Analog Converter (DAC) with dedicated conversion timer and DMA support.

> Power control:

- Four reduced power modes: Sleep, Deep-sleep, Power-down, and Deep power-down.

- The Wake-up Interrupt Controller (WIC) allows the CPU to automatically wake up from any priority interrupt that can occur while the clocks are stopped in Deep-sleep, Power-down, and Deep power-down modes.

- Processor wake-up from Power-down mode via any interrupt able to operate during Power-down mode (includes external interrupts, RTC interrupt, PORT0/2 pin interrupt, and NMI).

- Brownout detect with separate threshold for interrupt and forced reset.

- On-chip Power-On Reset (POR).

$>$ Clock generation:

- Clock output function that can reflect the main oscillator clock, IRC clock, RTC clock, CPU clock, USB clock, or the watchdog timer clock.

- On-chip crystal oscillator with an operating range of $1 \mathrm{MHz}$ to $25 \mathrm{MHz}$.

- $12 \mathrm{MHz}$ Internal RC oscillator (IRC) trimmed to $1 \%$ accuracy that can optionally be used as a system clock.

- An on-chip PLL allows CPU operation up to the maximum CPU rate without the need for a high-frequency crystal. May be run from the main oscillator or the internal RC oscillator.

- A second, dedicated PLL may be used for USB interface in order to allow added flexibility for the Main PLL settings.

Versatile pin function selection feature allows many possibilities for using onchip peripheral functions.

$>$ Unique device serial number for identification purposes.

> Single $3.3 \mathrm{~V}$ power supply (2.4 $\mathrm{V}$ to $3.6 \mathrm{~V}$ ). Temperature range of $40 \mathrm{C}$ to $85 \mathrm{C}$.

> Available as LQFP208, TFBGA208, TFBGA180, and LQFP144 package. 
ANEXO E - Extratos do datasheet do FS312F-P

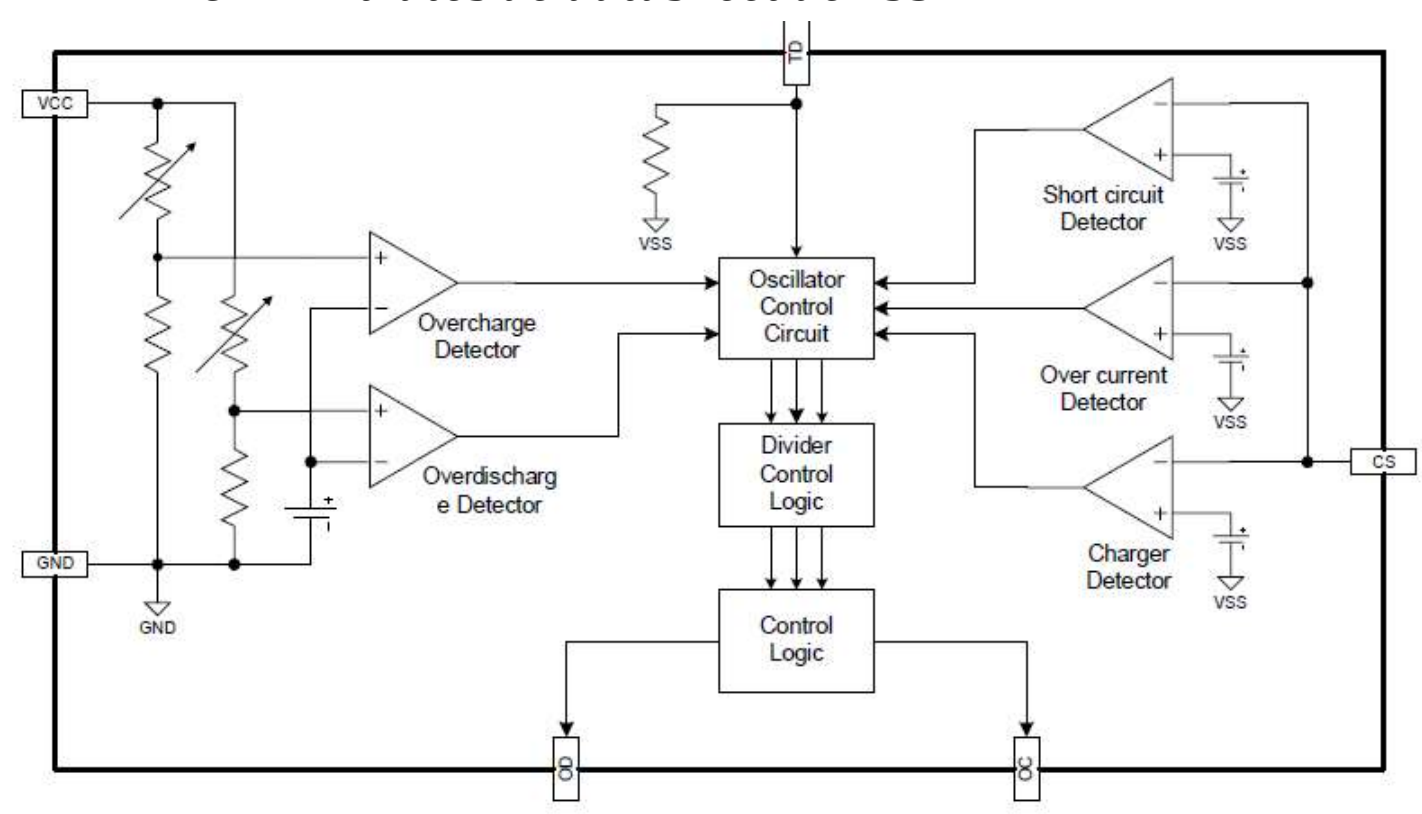




\begin{tabular}{|c|c|c|c|c|c|c|}
\hline PARAMETER & TEST CONDITIONS & SYMBOL & Min & Typ & Max & UNIT \\
\hline Supply Current & $\mathrm{VCC}=3.9 \mathrm{~V}$ & ICC & & 3.0 & 6.0 & $\mu \mathrm{A}$ \\
\hline Power-Down Current & $\mathrm{VCC}=2.0 \mathrm{~V}$ & IPD & & & 0.1 & $\mu \mathrm{A}$ \\
\hline Overcharge Protection Voltage & & VOCP & 4.225 & 4.25 & 4.275 & v \\
\hline Overcharge Release Voltage & & VOCR & 4.095 & 4.145 & 4.195 & v \\
\hline Overdischarge Protection Voltage & & VODP & 2.82 & 2.90 & 2.98 & v \\
\hline Overdischarge Release Voltage & & VODR & 2.92 & 3.00 & 3.08 & v \\
\hline Overcurrent Protection Voltage & & $\begin{array}{l}\text { VOIP } \\
\text { (VOI1) }\end{array}$ & 120 & 150 & 180 & $\mathrm{mV}$ \\
\hline Short Current Protection Voltage & $V C C=3.6 \mathrm{~V}$ & \begin{tabular}{|l} 
VSIP \\
(VOI2)
\end{tabular} & 1.00 & 1.35 & 1.70 & v \\
\hline Overcharge Delay Time & & TOC & & 200 & 300 & ms \\
\hline Overdischarge Delay Time & $V C C=3.6 \mathrm{~V}$ to $2.0 \mathrm{~V}$ & TOD & & 40 & 100 & $\mathrm{~ms}$ \\
\hline Overcurrent Delay Time (1) & $V C C=3.6 \mathrm{~V}$ & TOl1 & & 10 & 20 & $\mathrm{~ms}$ \\
\hline Overcurrent Delay Time (2) & $\mathrm{VCC}=3.6 \mathrm{~V}$ & TOI2 & & 5 & 50 & $\mu \mathrm{s}$ \\
\hline $\begin{array}{l}\text { Charger Detection Threshold } \\
\text { Voltage }\end{array}$ & & VCHA & -1.2 & -0.7 & -0.2 & v \\
\hline OD Pin Output " $\mathrm{H}$ " Voltage & & VDH & vec-0.1 & VCC- 0.02 & & v \\
\hline OD Pin Output "L" Voltage & & VDL & & 0.1 & 0.5 & v \\
\hline OC Pin Output "H" Voltage & & $\mathrm{VCH}$ & vcc- 0.1 & VCC-0.02 & & v \\
\hline OC Pin Output "L" Voltage & & VCL & & 0.1 & 0.5 & v \\
\hline
\end{tabular}

